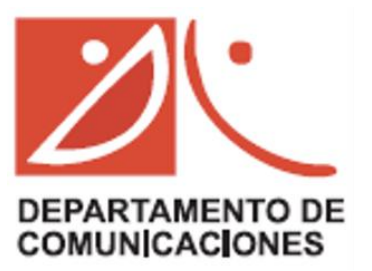

Universitat Politècnica de València

Departamento de Comunicaciones

\title{
Development of An Integrated Silicon Photonic Transceiver For Access Networks
}

\author{
Mariam Aamer
}

Supervisor: Dr. Pablo Sanchis Kilders

Tesis presentada para la obtención del grado de Doctor en Telecomunicaciones de la Universitat Politècnica de València.

Thesis submitted to the Universitat Politècnica de València in partial fulfillment of the requirements for the degree of Doctor of Philosophy in Telecommunications engineering. 



\section{Acknowledgment}

Puesto que esta tesis no es el resultado de un trabajo individual, me gustaría agradecer a todos (espero no dejarme a nadie, y si así fuera, pido disculpas por adelantado) los que de una manera u otra me han ayudado a llegar a este punto.

Gracias Javier por darme la oportunidad de formar parte del equipo del NTC, donde durante estos años he conocido a gente maravillosa.

Gracias muy especiales a Pablo, por supervisar mi trabajo durante este tiempo, por creer en mí, y por darme ánimos cuando las cosas se complicaban. Muchas gracias, has sido un gran director de esta tesis.

A Andreas, por compartir horas de laboratorio con todo el buen humor nórdico que las circunstancias permitían.

A JoseVi, por ser mi co-dire durante mi primera fase aquí, y por ser un gran amigo después.

A Nikos por su excelente trabajo y amabilidad durante mi estancia en Eindhoven.

A Antou, por ser un grandísimo compañero, por tu disponiblidad, por tu ayuda, por tus bromas, por ser una referencia.

A mis compañeros y compañeras de sala por la compañía, y por hacer más llevaderos estos últimos meses en los que los nervios han estado a flor de piel (Pak, JaviG, Josema, Jesús, Guillermo, Mercé, Dani, David, Amadeu y un largo etcétera).

A Josete, que aunque lo conocí en el ambiente laboral, es un amigo, un gran amigo que me llevo de mi trayecto en el NTC. Gracias porque me has animado cuando más lo necesitaba, porque has creído en mí, y por ser como eres. No cambies!!!

A mi compañera de trabajo, de confidencias, de bipos y mucho más, Ana. Simplemente Gracias neni.

Fuera del ambiente laboral, tengo la enorme suerte de tener que agradecer a muchas personas su apoyo incondicional desde el principio y en cada una de las dificultades o tropiezos que han surgido, y que no han sido pocos. Gracias a Kike, por compartir gran 
parte de este recorrido. Gracias a Anuski por enseñarme lecciones que solamente la vida nos puede enseñar.

A Nelson y Ari. A Irene, a la que conocí el primer día que pisé la escuela de Teleco, y no pensaba que con esa personita iba a compartir tanto, y lo que nos queda, espero.

A mis compañeras de equipo y a Mariví, por supuesto, por ser parte de mi familia, por hacerme disfrutar y sonreír cuando las circunstancias invitaban a tirar la toalla y salir corriendo. Lupis, tú eres una más de nosotras, gracias.

A mis mamis favoritas, y a la pixi; a mi "colega" Lauri (y a Pilar) simplemente por todo lo compartido. A mis Senents (Mire, sigo queriendo ser como tú de mayor; Fres, se te echa mucho de menos, y se te quiere aún en la distancia; A Rita, que suficiente que no me haya olvidado de ella; y a la maligna por excelencia por dejarme aprender algo de ella).

A mi hermana de otra sangre, por creer en mí más de lo que nadie se imagina, por entenderme hasta cuando ni yo me entendía, por su apoyo, por todo, gracias infinitas.

A Eva, eres un tesoro, un regalo que he tenido la enorme suerte de que entrara en mi vida, y que estoy segura que permanecerá en ella siempre. Me faltan palabras para agradecerte tu humanidad, empatía, implicación, sensibilidad, apoyo, por hacer que crezca con cada contratiempo. Gracias, muchas gracias de corazón.

A mi padre por demostrarme que está orgulloso de mí cada vez y que nunca es tarde.

A mis hermanos, por enseñarme muchas más cosas de las que he verbalizado nunca. Sois los mejores hermanos que he podido tener.

Y por último, a mi madre, la que le diga lo que le diga, me quedaré corta, muy corta para agradecerle todo. Has sido, eres y serás para mí un ejemplo de lucha, de entrega, de fortaleza, de humanidad, de vocación y amor por lo que una hace... Eres la mejor madre que he podido tener en esta y todas las posibles vidas que tuviera. Gracias por aguantar mis malos humos cuando los he tenido, y no han sido pocos, por darme aliento $\mathrm{y}$ animarme a seguir luchando por mi felicidad cuando lo quería abandonar todo. Por apoyarme aun cuando no estabas de acuerdo. Por la complicidad, la ternura, la alegría que me entregas a pesar de la distancia. Nunca te estaré lo suficientemente agradecida. Simplemente, GRACIAS pequeña. Te quiero. 


\section{Resumen}

Debido a la imparable aparición de dispositivos móviles multifunción junto con aplicaciones que requieren cada vez más un mayor ancho de banda en cualquier momento y en cualquier lugar, las futuras redes de acceso deberán ser capaces de proporcionar servicios tanto inalámbricos como cableados. Es por ello que una solución a seguir es el uso de sistemas de comunicaciones ópticas como medio de transporte de señales inalámbricas en enlaces de radio sobre fibra. Con ello, se converge a un dominio óptico reduciendo y aliviando el cuello de botella entre los estándares de acceso inalámbrico y cableado.

En esta tesis, como parte de los objetivos establecidos en el proyecto europeo HELIOS en el que está enmarcada, se han investigado y desarrollado los bloques funcionales básicos necesarios para realizar un transceptor fotónico integrado trabajando en el rango de longitudes de onda milimétricas, y haciendo uso de los formatos de modulación más robustos y que mejor se adaptan al ámbito de aplicación considerado.

El trabajo que se presenta en esta tesis se puede dividir básicamente en tres partes. La primera de ellas ofrece una descripción general de los beneficios del uso de la fotónica en silicio para el desarrollo de enlaces inalámbricos a velocidades de Gbps, así como el estado del arte de los transceptores desarrollados por los grupos de investigación más activos y punteros para satisfacer las necesidades de mercado, cada vez más exigentes.

La segunda parte se centra en el estudio y desarrollo del transmisor integrado de onda milimétrica. Primero realizamos una breve introducción teórica tanto del funcionamiento de los dispositivos que forman parte del transmisor, como a los formatos de modulación existentes, centrando la atención en la modulación por desplazamiento de fase (PSK) que es la que se va a utilizar en el desarrollo de los dispositivos implicados, y más concretamente en la modulación (diferencial) de fase en cuadratura ((D)QPSK). También se presentan los bloques básicos que integran nuestro transmisor y se fijan las especificaciones que deben cumplir dichos bloques para conseguir una transmisión libre de errores. El transmisor está compuesto por un filtro/demultiplexor encargado de separar dos portadoras ópticas separadas una frecuencia de $60 \mathrm{GHz}$. Una de estas portadoras es modulada al pasar por un modulador DQPSK basado en una estructura de dos MachZehnders (MZs) anidados, para ser nuevamente combinada con la otra portadora óptica 
que se ha mantenido intacta. Una vez combinadas, éstas son fotodetectadas para ser transmitidas inalámbricamente.

En la tercera parte de esta tesis, se investiga el uso de un esquema de diversidad en polarización junto a un receptor DQPSK integrado para la demodulación de la señal recibida. El esquema de diversidad en polarización está formado básicamente por dos bloques: un separador de polarización con el objetivo de separar la luz a la entrada del chip en sus dos componentes ortogonales; y un rotador de polarización.

En lo que se refiere al receptor DQPSK propiamente dicho, se ha investigado y optimizado cada uno de los bloques funcionales que lo componen. Éstos son básicamente un divisor de potencia termo-ópticamente sintonizable basado en un interferómetro MZ, en serie con un interferómetro $\mathrm{MZ}$ que introduce un retardo de duración de un bit en uno de sus brazos, para obtener una correcta demodulación diferencial. El siguiente bloque que forma parte de nuestro receptor DQPSK es un $2 \times 4$ acoplador de interferencia multimodal actuando como un híbrido de 90 grados, cuyas salidas van a parar a dos fotodetectores balanceados de germanio.

Las contribuciones principales de esta tesis han sido:

- Demostración de un filtro/demultiplexor con tres grados de sintonización con una relación de extinción superior a $25 \mathrm{~dB}$.

- Demostración de un rotador con una longitud de tan sólo $25 \mu \mathrm{m}$ y CMOS compatible.

- Demostración de un modulador DPSK a una velocidad máxima de $20 \mathrm{Gbit} / \mathrm{s}$.

- Demostración de un demodulador DQPSK a una velocidad máxima de $20 \mathrm{Gbit} / \mathrm{s}$. 


\section{Resum}

Degut a la imparable aparició de dispositius mòbils multifunció junt amb aplicacions que requereixen cada vegada més una major amplària banda en qualsevol moment i en qualsevol lloc, les futures xarxes d' accés deuran ser capaços de proporcionar servicis tant inalàmbrics com cablejats. És per açò que una solució a seguir és l' ús de sistemes de comunicacions òptiques com mitjà de transport de senyals inalàmbriques en enllaços de ràdio sobre fibra. Amb açò, es convergeix a un domini òptic reduint i alleujant el coll de botella entre els estàndards d' accés inalàmbric i cablejat.

En aquesta tesi, com part dels objectius establerts en el projecte europeu HELIOS en el què està emmarcada, s' han investigat $i$ desenvolupat els blocs funcionals bàsics necessaris per realitzar un transceptor fotònic integrat treballant en el rang de longituds d' ona milimètriques, i fent ús dels formats de modulació més robusts i que millor s' adapten a l' àmbit d' aplicació considerat.

El treball que es presenta en aquesta tesi es pot dividir bàsicament en tres parts. La primera d' elles ofereix una descripció general dels beneficis de l' ús de la fotònica en silici per al desenvolupament d' enllaços inalàmbrics a velocitats de Gbps, així com l' estat de l' art dels transceptors desenvolupats pels grups d' investigació més actius i capdavanters per a satisfer les necessitats de mercat, cada vegada més exigents.

La segona part se centra en l' estudi i desenvolupament del transmissor integrat d' ona milimètrica. Primer realitzem una breu introducció teòrica tant del funcionament dels dispositius que formen part del transmissor, com els formats de modulació existents, centrant l' atenció en la modulació per desplaçament de fase (PSK) que és la que s' utilitzarà en el desenvolupament dels dispositius implicats, i més concretament en la modulació (diferencial) de fase en quadratura ((D)QPSK). També es presenten els blocs bàsics que integren el nostre transmissor i es fixen les especificacions que deuen complir eixos blocs per aconseguir una transmissió lliure d' errades. El transmissor està compost per un filtre/demultiplexor encarregat de separar dos portadores òptiques separades una freqüència de $60 \mathrm{GHz}$. Una d' aquestes portadores es modulada al passar per un modulador DQPSK basat en una estructura de dos Mach-Zehnders (MZs) niats, per a ser 
novament combinada amb 1' altra portadora òptica que s' ha mantingut intacta. Una vegada combinades, aquestes són fotodetectades per a ser transmeses inalàmbricament.

En la tercera part d' aquesta tesi, s' investiga l' ús d' un esquema de diversitat en polarització junt a un receptor DQPSK integrat per a la demodulació del senyal rebut. L' esquema de diversitat en polarització està format bàsicament per dos blocs: un separador de polarització amb l' objectiu de separar la llum a l' entrada del xip en els seus dos components ortogonals; i un rotador de polarització.

Referent al receptor DQPSK pròpiament dit, s' ha investigat i optimitzat cada un dels blocs funcionals que ho componen. Aquestos són bàsicament un divisor de potència termo-òpticament sintonitzable basat en un interferòmetre $\mathrm{MZ}$, en sèrie $\mathrm{amb}$ un interferòmetre $\mathrm{MZ}$ que introdueix un retard de durada d' un bit en un dels seus braços, per a obtenir una correcta demodulació diferencial. El següent bloc que forma part del nostre receptor DQPSK és un $2 \mathrm{x} 4$ acoblador d' interferència multimodal actuant com un híbrid de 90 graus, les eixides del qual van a parar a dos fotodetectors balancejats de germani.

Les contribucions principals d' aquesta tesi han sigut:

- Demostració d' un filtre/demultiplexor amb tres graus de sintonització amb una relació d' extinció superior a $25 \mathrm{~dB}$.

- Demostració d' un rotador amb una longitud de només $25 \mu \mathrm{m}$ i CMOS compatible.

- Demostració d' un modulador DPSK a una velocitat màxima de $20 \mathrm{Gbit} / \mathrm{s}$.

- Demostració d' un demodulador DQPSK a una velocitat màxima de $20 \mathrm{Gbit} / \mathrm{s}$. 


\section{Abstract}

Due to the relentless emergence of multifunction mobile devices with applications that require increasingly greater bandwidth at anytime and anywhere, future access networks must be capable of providing both wireless and wired services. The use of optical communications systems as transport medium of wireless signals over fiber radio links is a steady solution to be taken into account. This will make possible a convergence to an optical domain reducing and alleviating the bottleneck between wireless access standards and current wired access.

In this thesis, as part of the objectives of the European project HELIOS in which it is framed, we have investigated and developed the basic functional blocks needed to achieve an integrated photonic transceiver working in the range of millimetre wavelengths, and using robust modulation formats that best fit the scope considered.

The work presented in this thesis can be basically divided into three parts. The first one provides an overview of the benefits of using silicon photonics for the development of wireless links at rates of Gbps, and the state of the art of the transceivers reported by the most important research groups in order to meet the increasingly demanding needs' market.

The second part focuses on the study and development of millimetre-wave integrated transmitter. First we provide a brief theoretical introduction of the operation principles of the devices involved in the transmitter such as a modulation formats, focusing on the phase shift keying (PSK) which is the one that will be used, particularly the (differential) quadrature phase shift keying ((D) QPSK). We also present the building blocks involved in our transmitter and we set the specifications that must be met by these devices in order to achieve an error-free transmission. The transmitter includes a filter/demultiplexer which must separate two optical carriers $60 \mathrm{GHz}$ separated. One of these optical carriers is modulated by passing through a DQPSK Mach-Zehnder-based modulator (MZM) by arranging two MZMs in a nested configuration. Using a combiner, the modulated optical signal and the un-modulated carrier are combined and photodetected to be transmitted wirelessly.

In the third part of this thesis, we investigate the use of a polarization diversity scheme with an integrated DQPSK receiver for demodulating of the wireless signal. The 
polarization diversity scheme basically consists of two blocks: a polarization splitter in order to separate the random polarization state of the incoming light into its two orthogonal components, and a polarization rotator.

Regarding the DQPSK receiver itself, all the functional blocks that comprise it have been investigated and optimized. It basically includes a thermo-optically tunable $\mathrm{MZ}$ interferometer power splitter, in series with a MZ interferometer that introduces, in one of its arms, a delay of one bit length in order to obtain a correct differential demodulation. The next building block of our DQPSK receiver is a $2 \times 4$ multimode interference coupler acting as a 90 degree hybrid, whose outputs are connected to two balanced germanium photodetectors.

The main contributions of this thesis are:

- Demonstration of a filter/demultiplexer with three degrees of tuning and an extinction ratio greater than $25 \mathrm{~dB}$.

- Demonstration of a polarization rotator with a length of only $25 \mu \mathrm{m}$ and CMOS compatible.

- Demonstration of a DPSK modulator at a maximum rate of $20 \mathrm{Gbit} / \mathrm{s}$.

- Demonstration of a DQPSK demodulator to a maximum rate of $20 \mathrm{Gbit} / \mathrm{s}$. 


\section{Contents}

\section{Acknowledgment}

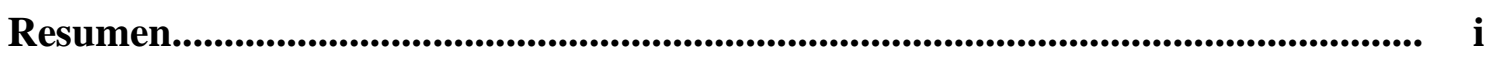

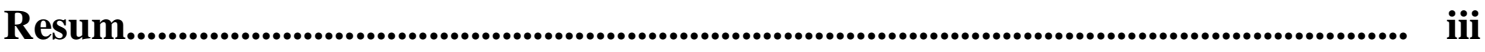

Abstract................................................................................................................................. $\quad \mathbf{v}$

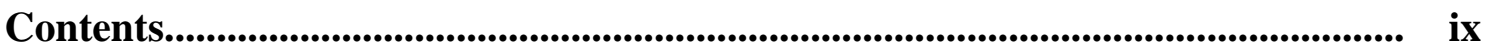

1. Introduction 1

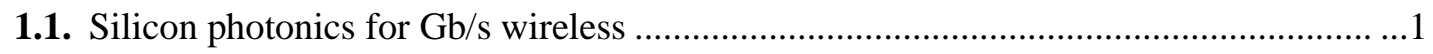

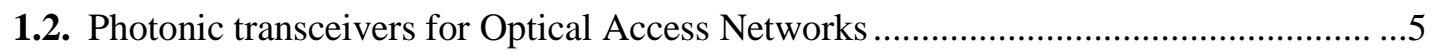

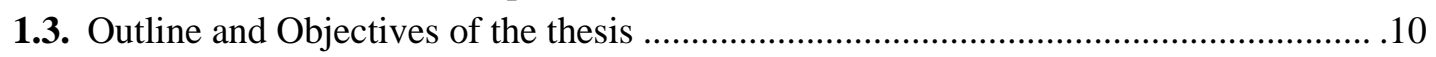

2. Transmitter 13

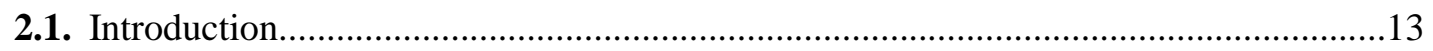

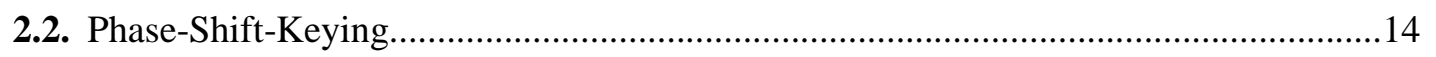

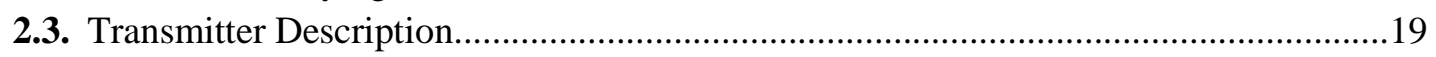

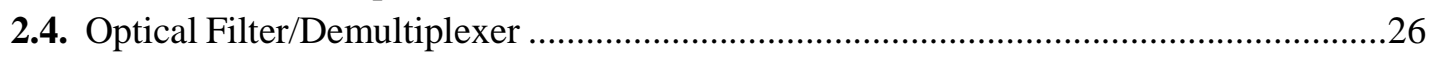

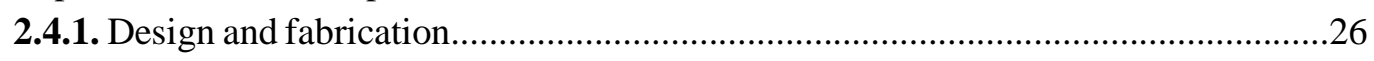

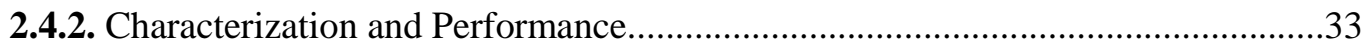

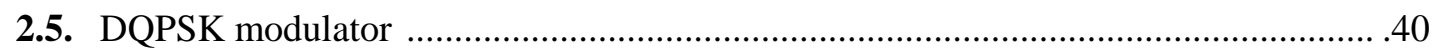

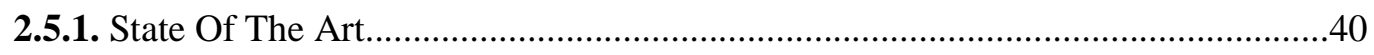

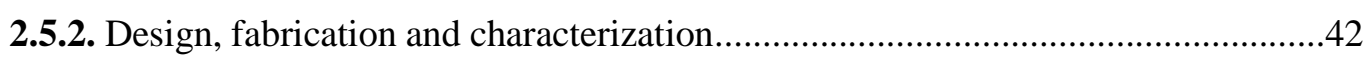

3. Receiver $\quad 49$

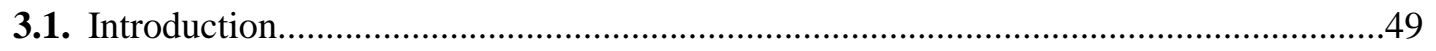

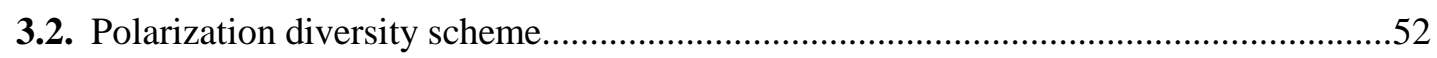

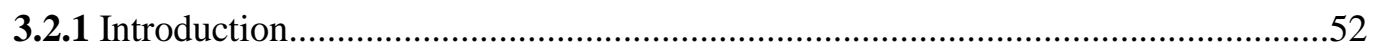

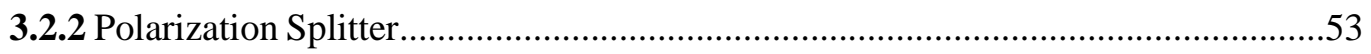

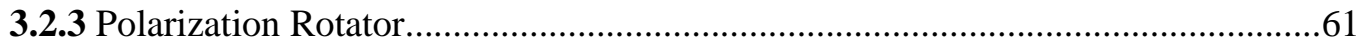

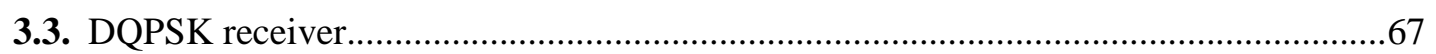

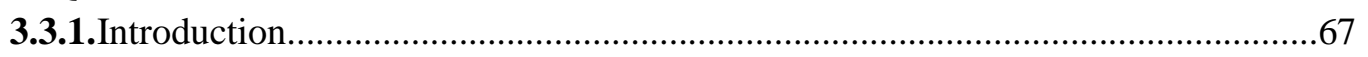

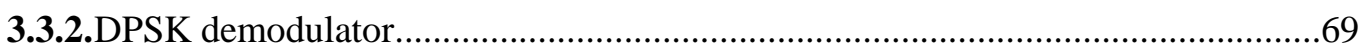

3.3.3.DQPSK Demodulator with Balanced Detection................................................... 75

4. Conclusions and future outlook $\mathbf{8 5}$ 
Appendix A

List of Publications

List of Figures

95

List of Tables

101

Acronyms

103

Bibliography

107 




\section{Chapter 1}

\section{Introduction}

\subsection{Silicon Photonics for Gbps Wireless}

Photonics, the science of generating, modulating, guiding, processing and detecting light, is a rapidly growing sector in the global economy, in which optical communications, optical storage, imaging, optical sensors or security are just a few examples.

Even if photonics could add new functionalities to electronic components such as low propagation losses, high bandwidth, wavelength multiplexing and immunity to electromagnetic noise, the high cost of photonic components and their assembly is a major obstacle to their deployment in most of application fields. Like in micro-electronics, many applications can be realized in a much more compact and cost-effective way by integrating the required functionality in a single chip.

As yet, the progress in photonic integration has been hampered by the huge variety in photonic devices and technologies, and the fact that the most integration technologies are specific for the application they have been developed for. As a result, the market for integrated photonics is too fragmented to justify the large investments for developing an integration technology to a level that really would really lead to substantial costs reduction, and in turn, to prevent a rapid growth of the applications.

Silicon photonics, or Complementary Metal-Oxide-Semiconductor (CMOS) Photonics, is a way to tackle the problem by the development of a small number of generic integration technologies with a level of functionality that can address a broad range of applications. These technologies, which should be made accessible via foundries, can betake to markets that are sufficiently large to pay back the developments costs. 
As in micro-electronics, the key for the success of integration in photonics is the realization of a broad range of optical functionalities with a reduced set of elementary components, and the development of a generic wafer-scale technology for integration. This generic technology will most likely not wipe away more specialized custom technologies but will create new opportunities for a much larger deployment of photonic integrated circuits (PICs). Because a single device will have an insignificant cost, designers should find the way to achieve the desired functions by using as many components as needed. Moreover, by co-integrating optics and electronics on the same chip, high performance, high functionality and highly integrated devices can be fabricated while maintaining the well-known and proven microelectronics fabrication process.

Another advantage of CMOS photonics is that its success will move the emphasis from the component level to the architecture one, which would imply that industrial and Research, Technology and Development (RTD) efforts could be focused on new products or new functionalities rather than on the technology level.

Proof of concept or functional demonstrations of many building blocks have been carried out in previous research projects. However, even though the obtained results were considered as a major breakthrough, major efforts are still being needed in order to improve the performance in terms of functionality, power or bandwidth. To go one step further, the European CMOS Photonics community has to demonstrate the integration of photonics with electronics and make available an integrated design and fabrication chain with generic and standard processes that could be transferred to foundries.

This thesis is framed in the European project called HELIOS [HEL] (pHotonics ELectronics functional Integration on CMOS). The HELIOS consortium has developed innovative means to combine a photonic layer with a CMOS circuit, using microelectronics fabrication processes. HELIOS has gathered 19 partners among the major European CMOS Photonics and Electronics players and potential users. It has driven the European RTD in CMOS Photonics and has paved the way for industrial development [HEL].

The objectives of HELIOS have been the following:

- Development of high performance generic building blocks that can be used for a broad range of applications such as wavelength-division multiplexing (WDM) sources by III-V/Si heterogeneous integration, fast modulators and detectors, passive circuits and packaging.

- Building and optimization of the whole "food chain" in order to fabricate complex functional devices.

- Demonstrating the power of this proposed "food chain" by realizing several complex PICs addressing different industrial needs, including a 40 Gbps modulator, a 10x10 Gbps transceiver, a photonic $10 \mathrm{Gbps}$ wireless transmission and a mixed analog and digital transceiver module for multifunction antennas. 
- Road mapping, dissemination and training to strengthen the European research and industry in this field and to raise awareness of new users about the interest of CMOS Photonics.

One of the silicon-based technologies that is experiencing great development is the called Silicon On Insulator (SOI) one. This technology is basically characterized by the high refractive index contrast between the two materials, where the refractive indices of the silicon (core) and the oxide (cover) at an operating wavelength of $1550 \mathrm{~nm}$ are 3.45 and 1.45 , respectively.

However, one of the main issues depending on the final applications the relatively high birefringence, which is defined as the difference between the effective indices of the fundamental mode of a certain structure, for both TE and TM polarizations is defined as follows:

$$
\Delta n_{e f f}=n_{e f f}^{T E}-n_{e f f}^{T M}
$$

The variation of this parameter ( $\Delta n_{\text {eff }}$ ) has several sources such as the intrinsic properties of the material itself [HUN84], the manufacturing process used in the fabrication of the waveguide [KAW90] or the asymmetry of the geometry of the waveguide itself [VAS91].

SOI waveguides are mostly asymmetric, and from electromagnetic theory [VAS91] it can deduced that birefringence increases with the asymmetry of the structure, as well as if the dimensions of the waveguides decreases.

The figure below shows the fundamental mode of a rectangular SOI waveguide, with the dimensions that will be used in this thesis. As it can be seen, the TE mode is more confined than the TM one, indicated also by the values of the corresponding effective index.

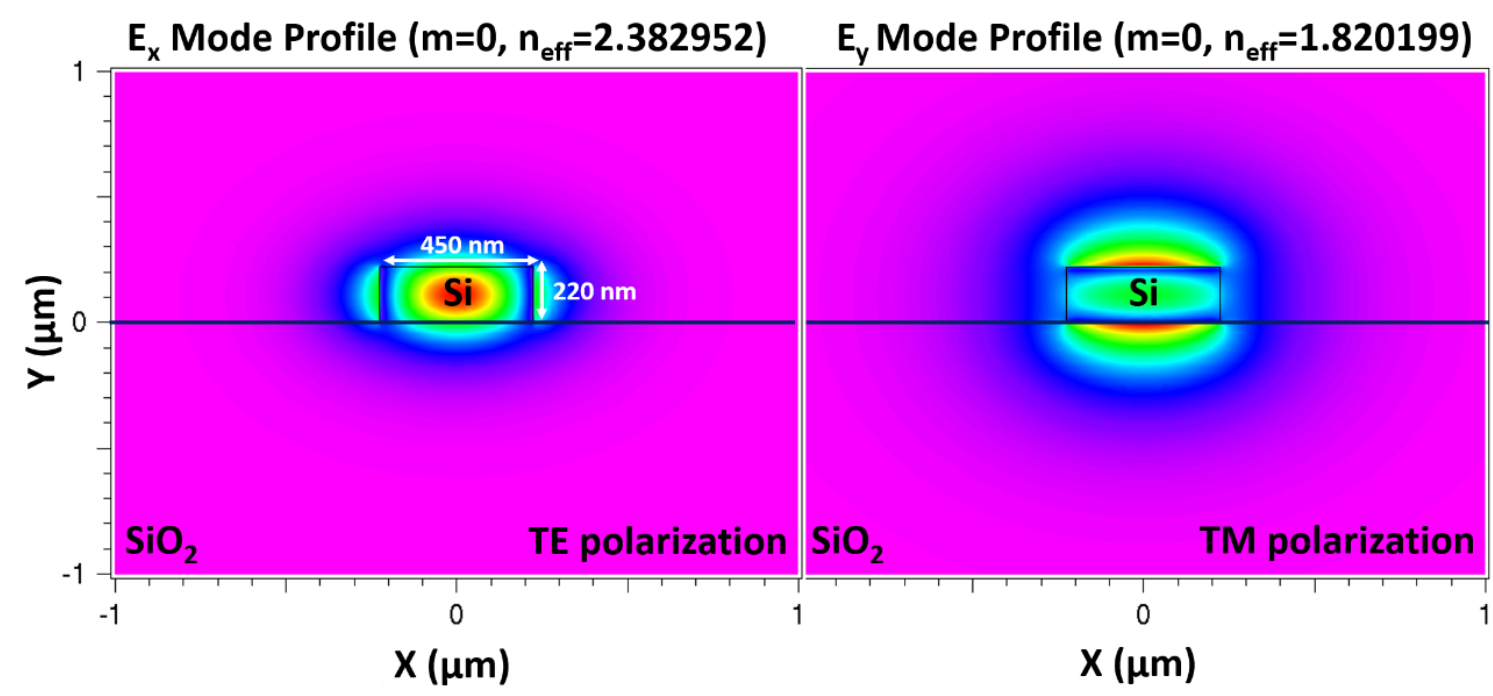

Figure 1.1: Aspect of the fundamental mode in a rectangular waveguide for the TE (left) and TM (right) polarization, and the values of the effective indices, resulting $\Delta n_{\text {eff }} \approx 0.57$. 
Broadband wireless access (BWA) networks are key last-mile-access technologies. BWA facilitates the extension network's coverage with relatively low deployment costs, faster revenue growth, and increased flexibility compared to common cabled infrastructure. On the other hand, wired local area networks (LAN) and fiber-to-the-home (FTTH) access networks are experiencing a remarkable capacity increase because the costs are decreasing due to higher market penetration, standardization, and the use of low-cost optical technologies. This increasing capacity of wired data transmission has pushed the development of wireless technologies capable of transmitting high data rate signals, which is only possible by using the millimeter wave $(\mathrm{mmW})$ band.

Specifically, the $60 \mathrm{GHz}$ band has drawn a lot of interest because it presents one of the largest unlicensed bandwidths being allocated in history, $9 \mathrm{GHz}(57-66 \mathrm{GHz})$ in Europe, and $7 \mathrm{GHz}(57-64 \mathrm{GHz})$ in the US. However, transmission using this band is also associated with both advantages and disadvantages.

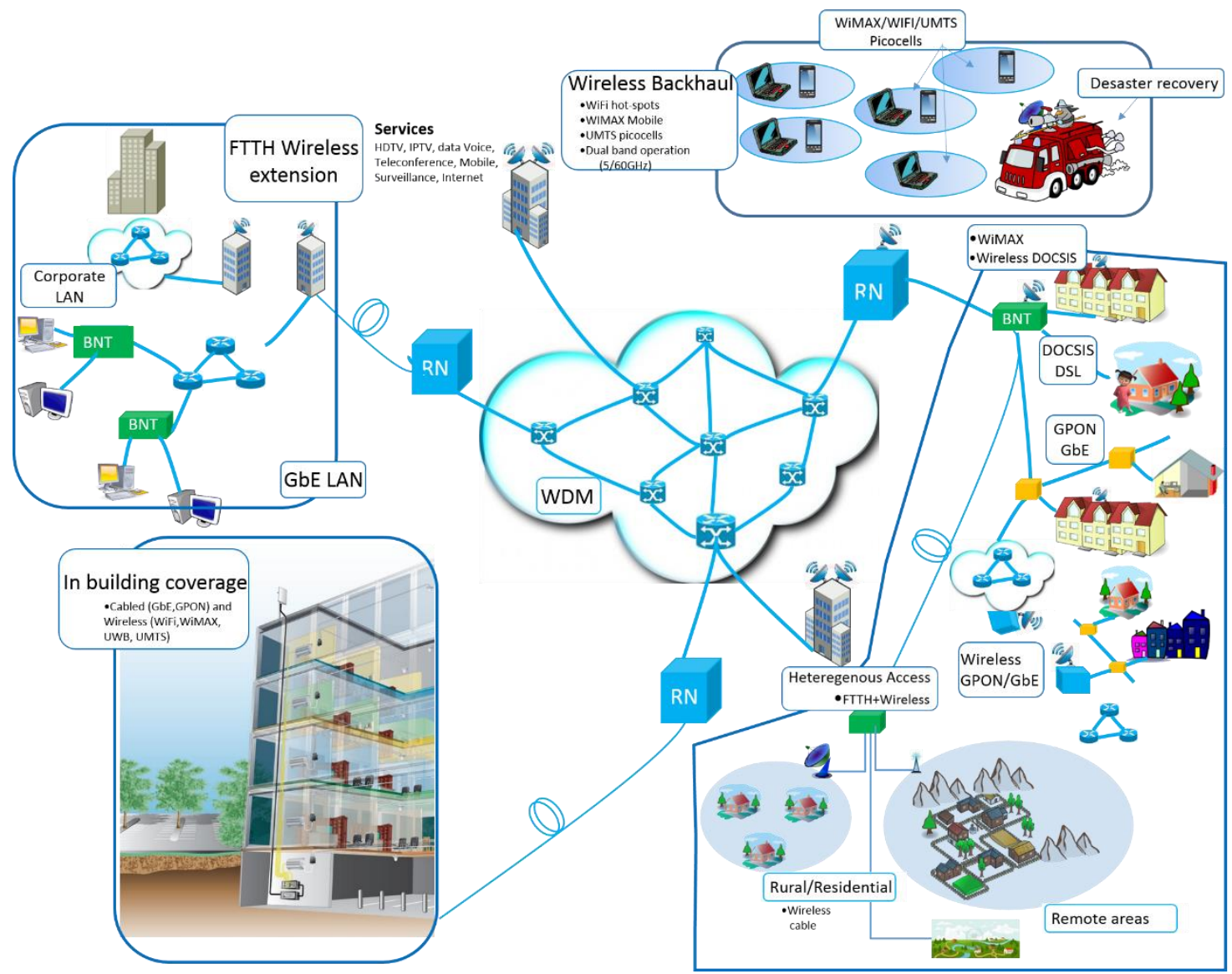

Figure 1.2: Artistic projection of the various application scenario of a gigabit wireless link.

High propagation loss due to oxygen absorption at this band make it unsuitable for long links and higher transmit power, but for it is suitable for short-range applications. Moreover, these frequencies lead not only to smaller sizes of RF components including antennas, but also they can be quite directional (coming with high antenna gain), which is highly desired. 
Even so, the $60 \mathrm{GHz}$ links capabilities with a mature electronics make them the natural choice for extending LANs between campus buildings, connecting enterprise sites into metro fiber backbones, and creating virtual fiber backbones and meshes whenever construction costs or delay make fiber installation unattractive. These links are also the technology of choice for providing truly independent redundancy for critical fiber connections in both private and public networks.

Different application scenarios have been identified for the $\mathrm{mmW}$ wireless architecture. Figure 1.2 shows a schematic of several wireless applications. Some of the most interesting scenarios are LAN connections (buildings, urban areas, enterprise connectivity), disaster recovery, last mile coverage of FTTH, connectivity where a direct fiber link costs are too high or impossible such as over highways, rivers or nature obstacles, redundancy links, and in battle field for real-time monitoring.

\subsection{Photonic Transceiver for Optical Access Networks}

Phase modulated mmW signal generation has attracted very strong interest in the last years and recently a modulator concept based on delay modulation to generate phase shift keying (PSK) modulation wireless signals has been demonstrated [DOI00]. Another way of generating phase modulated wireless signals is using single side-band (SSB) modulation, where two optical carriers are required to generate the RF signal, but only one of the optical carriers is phase modulated. When such a SSB signal is photodetected, the optical phase modulation is converted into a $\mathrm{mmW}$ phase modulated signal. One way of doing this is by filtering one of the optical signals and passing it through an optical modulator and the recombined signal will be SSB in nature as shown in Figure 1.3.

Several experimental demonstrations have been reported on the use of this technique [OZE01, WIB05] using common optical components like fibre Bragg's gratings and optical modulators like a Mach-Zehnder modulator (MZM). For phase modulation, the use of common components require that the two optical signals have phase and polarization correlation prior to photo-detection. This can be very difficult to implement since the optical carriers travel different paths, and for multi-level phase modulation the requirements on phase correlation increases.

Photonic integration of the optical modulator with the filters can provide the required phase and polarization control since the path lengths can be accurately controlled and the devices can be designed to maintain polarization. Optical devices are mostly made using III-V semiconductors, but recently silicon based optical devices has attracted a lot of interest for both digital and analogue applications [LIA05a, LIP06, PIN08, BRI12, MAN10]. The main advantage of silicon photonics technology is the possibility of monolithic integration of photonics and electronics using cost-effective CMOS technology [PIN08]. 


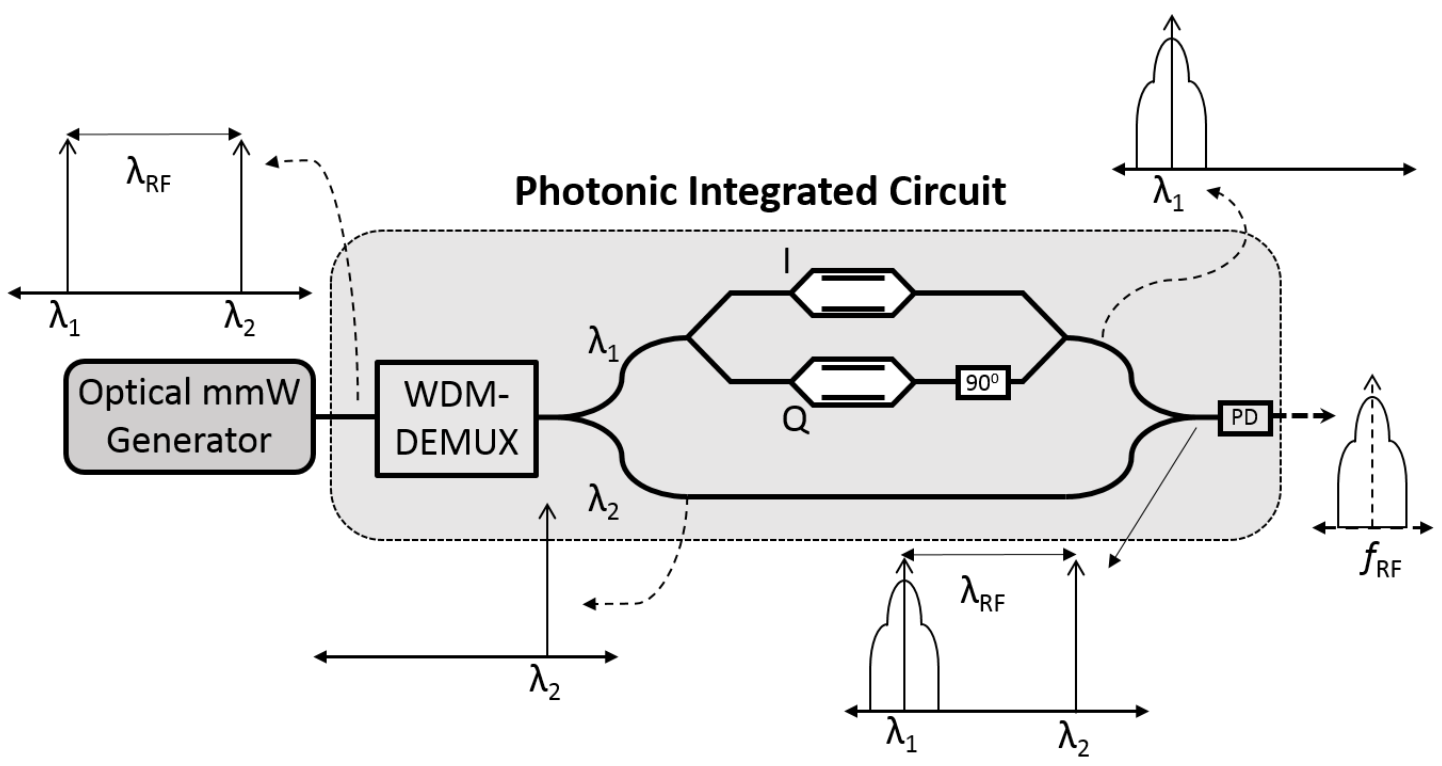

Figure 1.3: Illustration of a millimetre wave transmitter.

Optical modulators, photo-detectors, filters, and other optical components using Si have been demonstrated for FTTH networks aimed at reducing the cost [ZHA10a]. The wireless link consists of two wireless optical units, a transmitter and a receiver. The transmitter has an input and output fiber connections, as well as two RF connectors. The elementary components are fiber couplers, a tunable filter, a modulator (DQPSK-MZM) consisting of two 5 Gbps MZMs, and monitoring photodetectors. The second chip, the receiver, is connected to an optical fiber to receive the $10 \mathrm{Gbps}$ DQPSK signal. The demodulator includes fiber couplers, Mach-Zehnder delay interferometer (MZDI), and balanced photodetectors (BPDs). The output of the two BPDs is the two original $5 \mathrm{Gbps}$ RF signals.

PICs assembling a number of functional elements had been attracting large attention for both telecommunications and data-communications equipment providers. There are actually two main directions taken in the integration: i) monolithic integration on InP substrate, and ii) hybrid integration involving III-V for light emission and silicon for all the other functions. The first one leaded by Infinera company targets metropolitan and long-haul telecommunication applications. This approach allows the achievement of very good performances but at the expense of sophisticated III-V processing. The other approach is represented by a number of companies such as Luxtera and Intel, which is a low cost approach, and its target is more focused for optical interconnects for data communications of much shorter distance. This approach uses CMOS compatible process and hence, is suitable for mass production.

Regarding the monolithic approach, Infinera developed and published a transmitterreceiver for Nx10 Gbps and Nx40 Gbps [WEL07]. The figure below (Figure 1.4(a)) shows a schematic of the transmitter PIC device. Each channel has a laser and an electroabsorption (EA) modulator for encoding the data onto the laser output. Depending on the modulator design, data rates from $10 \mathrm{Gbps}$ to $40 \mathrm{Gbps}$ can be supported. 
The encoded optical signal is amplified by an integrated semiconductor optical amplifier (SOA). Each channel also has a power monitoring photodiode.

Figure 1.4(b) shows the architecture corresponding to the receiver PIC with a wide optical bandwidth SOA integrated at the PIC's input.

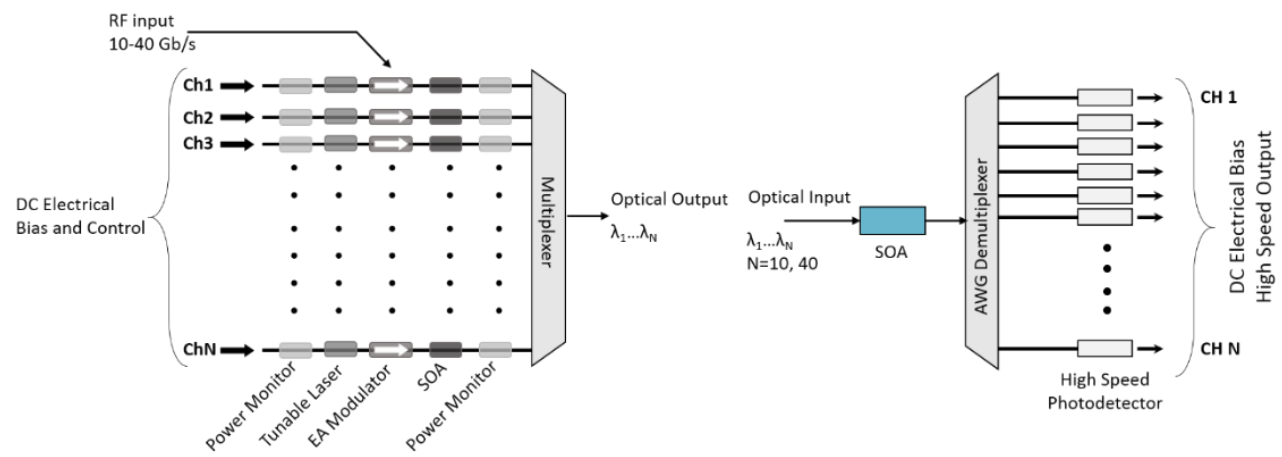

a) Transmitter

b) Receiver

Figure 1.4: Infinera's 10 and 40-channel transmitter and receiver integrating SOAs.

The input channels are then demultiplexed using an arrayed waveguide grating (AWG) router. Once the channels are demultiplexed, they pass through an array of high speed waveguide photodetectors. At Optical Fiber Conference (OFC) 2010, an InP PIC receiver for 10 channels, 45.6 Gbps per channel, polarization multiplexed DQPSK system was presented [NAG11]. This circuit incorporates a polarization beam splitter, AWG for wavelength demultiplexing, $90^{\circ}$ hybrids and balanced PDs.

On the other side, photonic integration in silicon has been mainly Luxtera and Intel. On 2007, Luxtera demonstrated a fully integrated, four channel, DWDM transceiver using interleaver optical multiplexers/demultiplexers achieving an aggregate data rate of 40 $\mathrm{Gb} / \mathrm{s}$ over a single fiber at a bit error ratio (BER) $<10^{-12}$ [NAR07].

In 2008, the first ever demonstration of a fully integrated and programmable $40 \mathrm{~Gb} / \mathrm{s}$ optical data communication system on a single SOI chip [DOB08] was reported. The die, shown in the figure below, includes high speed MZI modulators, low-loss waveguides, high efficiency grating couplers, tunable WDM multiplexers and demultiplexers, and monolithically integrated Ge-PIN waveguide photodetectors. The electronic receiver circuitry also includes high-speed transimpedance amplifiers and limiting amplifiers.

On September 2012, and based on the same technology platform, Luxtera reported [DOB12] a $4 \times 28 \mathrm{~Gb} / \mathrm{s}$ transceiver module, which introduced several optimizations to reduce the transmitter footprint, with very good performance. 
a)

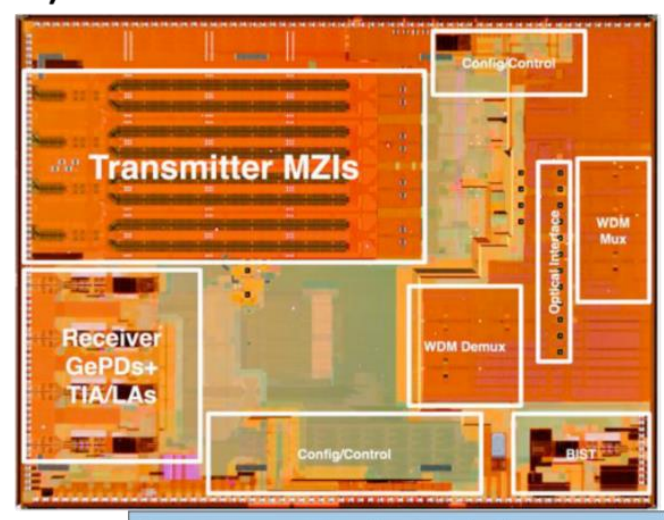

Single, inexpensive $1550 \mathrm{~nm}$ directly attached laser
Illuminates all four lanes with unmodulated light

c)

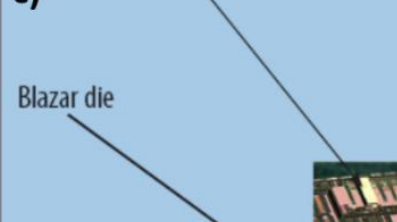

4 X 10 Gbps MZI modulators fabricated monolithically from silicon b)

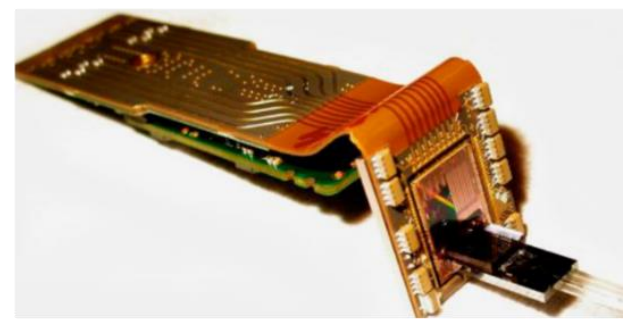

Figure 1.5: (a) Luxtera's photograph of CMOS $4 \times 10 \mathrm{~Gb} / \mathrm{s}$ WDM die (b) on flexible circuit and fiber-array. (c) Luxtera integrations scheme for transceiver $4 \times 10 \mathrm{~Gb} / \mathrm{s}$

As commented previously, Intel shares the PICs assembling approach with Luxtera, and is actively engaged in the development of these PICs for optical interconnects. On July 2010, Intel announced an integrated silicon photonic transmitter using hybrid silicon lasers that is capable of sending data at $50 \mathrm{~Gb} / \mathrm{s}$ across an optical fiber to an integrated silicon photonic receiver chip which converts the optical data back into electrical [INT].

In order to achieve 50Gb/s, 4 wavelengths $(1351,1331,1311$ and $1291 \mathrm{~nm})$ were used, each carrying $12.5 \mathrm{~Gb} / \mathrm{s}$ modulated signal through a silicon modulator. A multiplexer is then used to combine the optical channels and launch them into a fiber via an on-chip fiber coupler. On the receiver chip, optical signals received and separated by a 4 channel demultiplexer, are then directed into four integrated germanium photodetectors. Figure 1.6 shows two photographs of the $50 \mathrm{~Gb} / \mathrm{s}$ Intel's transmitter and receiver modules.

In January 2013, Intel and Facebook began a collaboration on a new disaggregated, rackscale server architecture based on the technology commented above [INT13]. 

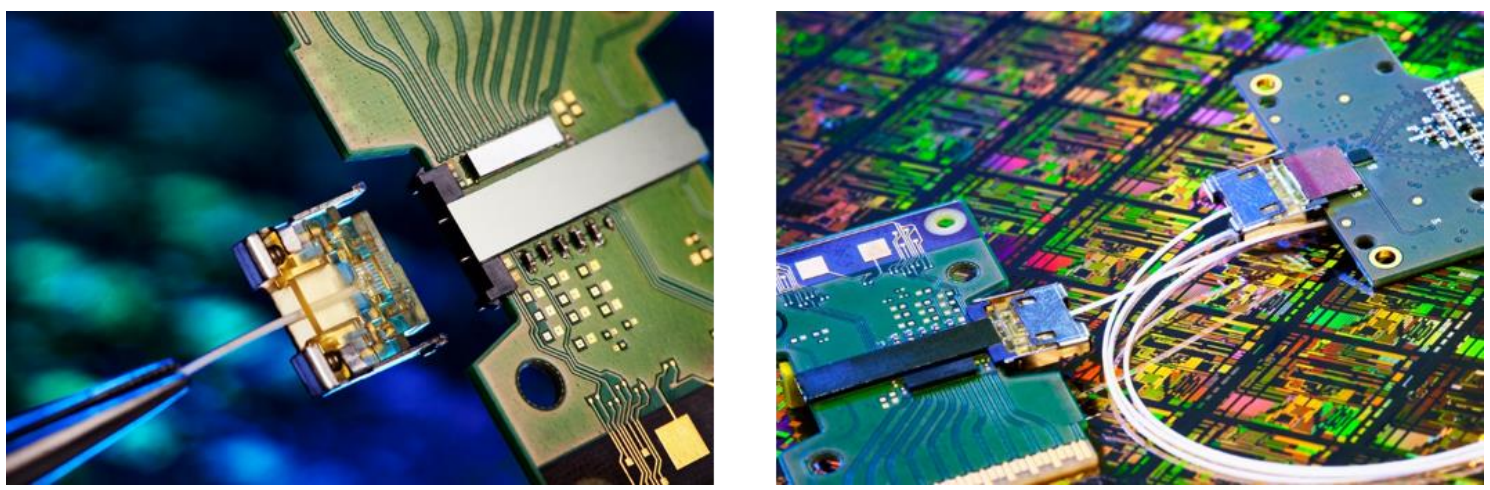

Figure 1.6: Picture of Intel's $50 \mathrm{~Gb} / \mathrm{s}$ transmitter and receiver. The transmitter includes hybrid IIIV/SOI lasers using wafer bonding and a wavelength multiplexer, the receiver includes a wavelength demultiplexer and Ge/Si photodetectors [INT].

Kotura, which will be acquired by Mellanox Technologies in the second half of 2013 [KOT13], is another actor in transceivers fabrication. The figure below shows a schematic a SEM images of a $4 \times 25 \mathrm{~Gb} / \mathrm{s}$ transmitter and receiver. One of the two chips is the silicon transmitter which houses the platform for the four lasers combined as a four-channel array. Each is an external cavity laser where part of the cavity is within the InP device and the rest in the silicon photonics waveguide. The gain chips are flip-chipped onto the silicon. The transmitter also includes a grating that sets each laser's wavelength, four modulators, and a WDM multiplexer to combine the four wavelengths before transmission on the fibre. The receiver chip uses a four-channel demultiplexer with each channel fed to a Ge-PD. Two chips are used as it is easier to package each as a transmitter optical sub-assembly (TOSA) or receiver optical sub-assembly (ROSA). According to Kotura's forecasts, the 100Gbps Quad Small Form-factor Pluggable (QSFP) will be generally available in 2014 .

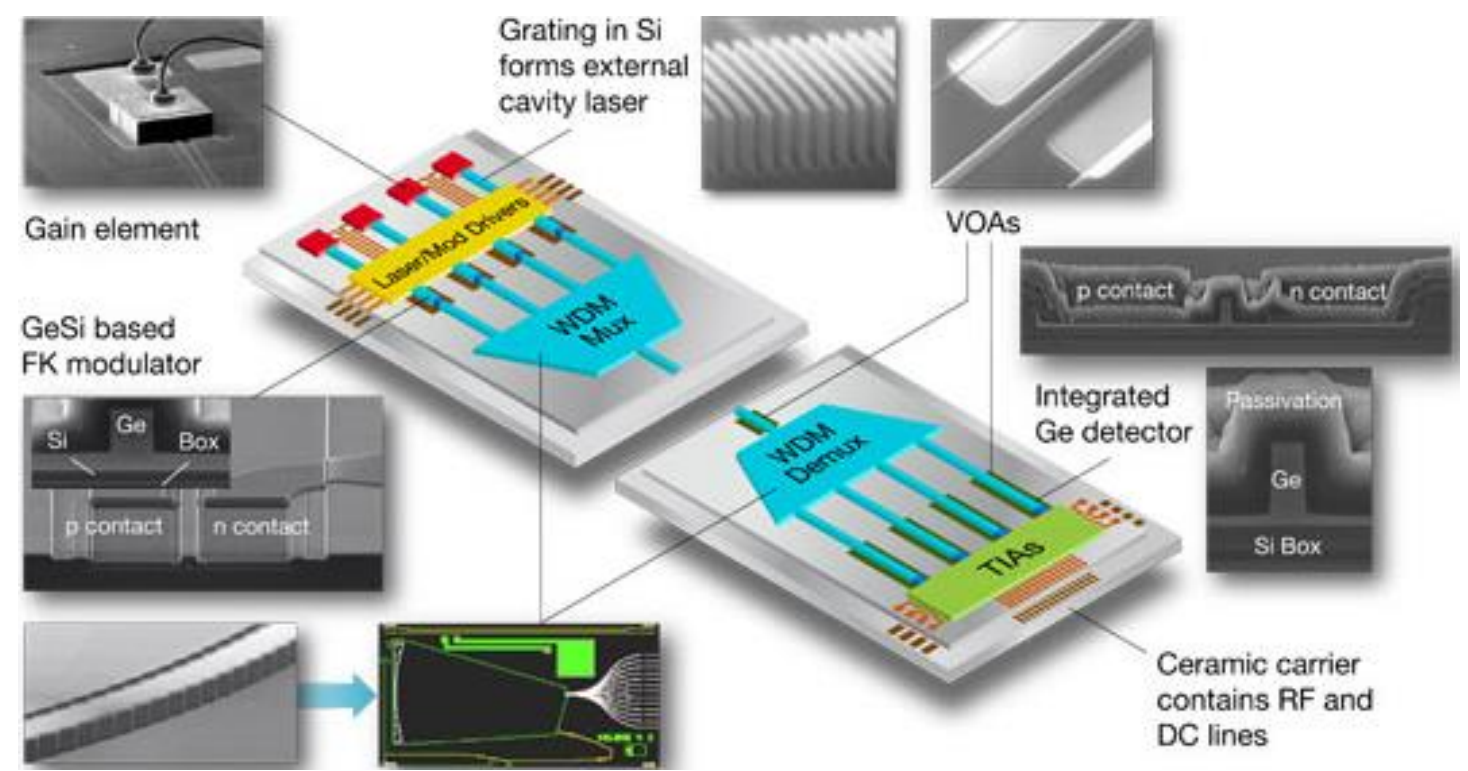

Figure 1.7: Kotura's 4x25Gb/s transmitter and receiver chips [KOT].

Another player in this scene is Oracle Labs. A fully integrated silicon photonic transceiver was demonstrated on June 2012 [BUC12] in SOI process using photonic microring 
resonator-based modulators, operating at data rates of $25 \mathrm{~Gb} / \mathrm{s}$ with a BER of $10^{-12}$. Figure 1.8 shows a microphotograph of the reported $25 \mathrm{~Gb} / \mathrm{s}$ optical transceiver.

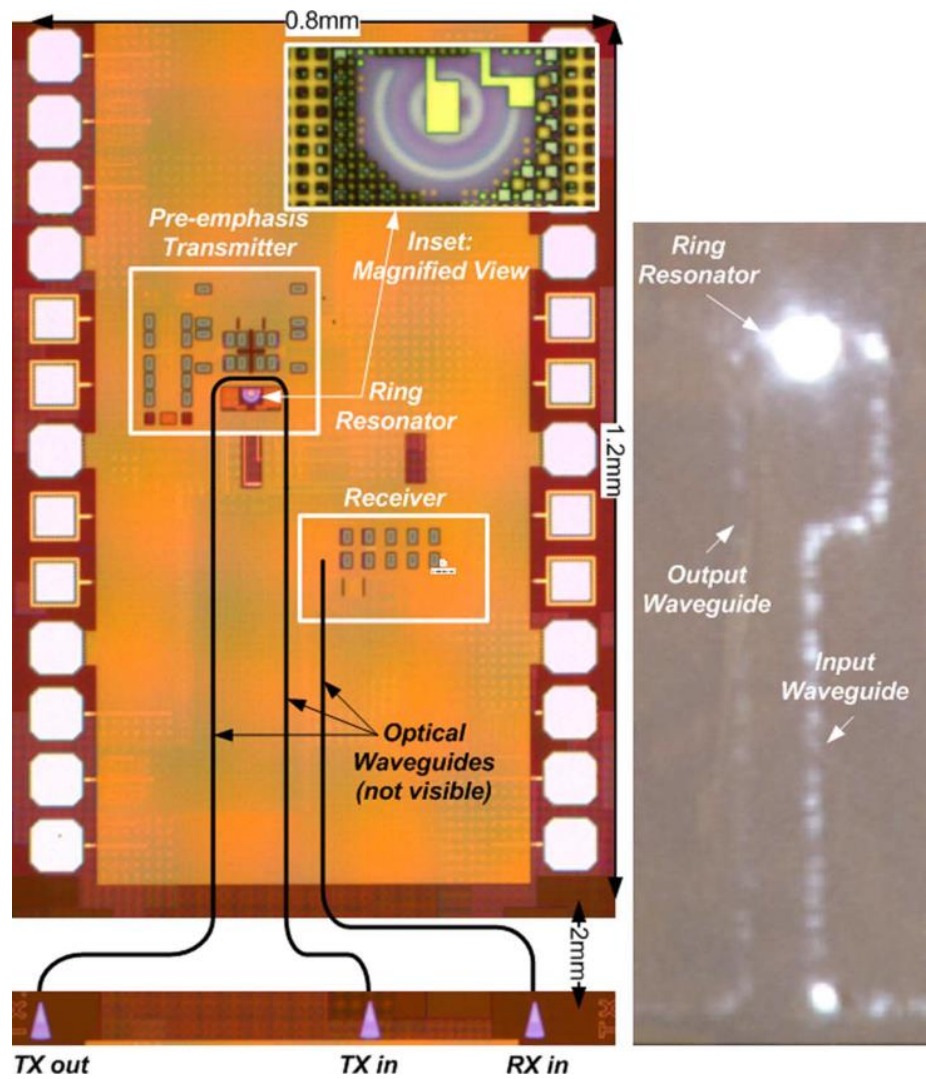

Figure 1.8: (Left) Chip microphotograph of the $25 \mathrm{~Gb} / \mathrm{s}$ optical transceiver. At the bottom, waveguide couplers are shown on the same silicon wafer. Two millimetres between the couplers and the active circuits is omitted. (Right) an infrared image of the chip shows light leakage from silicon photonic waveguide and absorption in ring modulator [BUC12].

One of the most recent news which has appeared less than two months ago and demonstrates the present interest in developing optical transceivers is [CIS] concerning Cisco and its CPAK 100G silicon photonics-based optical transceiver after the acquisition of the CMOS silicon photonics developer Lightwire in the third quarter of 2012 [LIG].

\subsection{Outline and Objectives of the thesis}

The demand for optical links at high volume, with low cost, low power and high reliability, is becoming increasingly evident as electrical interconnects struggle to cope with the demands of even short range connectivity at speeds beyond $10 \mathrm{Gbps}$. By taking advantage of the vast investments made by the semiconductor industry, silicon photonics allows high-volume, high yield and low-cost manufacturing of complex photonic integrated circuits. The emergence of mobile devices such as multifunction mobile phones and tablets accompanied with near-future bandwidth insensitive applications (such as Ultra High Definition TV data/3D Internet and Hi-Vision, and interactive 3D video applications, among others) has become one of the drivers for demanding wireless 
data capacity on the scale of the $10 \mathrm{Gbps}$. Both optical and wireless networks have significant strengths and noticeable weaknesses. While photonic technologies provide much higher bandwidth and support long transmission links, they require costly hard lines. Conversely, wireless technologies are not suitable for sending large amounts of information to many users with high quality of service. Therefore, it is highly desirable that the future wireless link will possess the same capacity with the optical fibers to realize the seamless hybrid fiber-wireless access over the last mile, in order to optimally serve end users conveniently and cost effectively through a hybrid solution integrating both optical and wireless technologies.

The aim of this thesis is to provide a demonstrator of a photonic transmitter and its corresponding transparent polarization receiver, in the millimetre wave frequency range, by making use of a spectral efficient and robust modulation format. The building blocks concerning the transmitter and the receiver are depicted in the figure below.

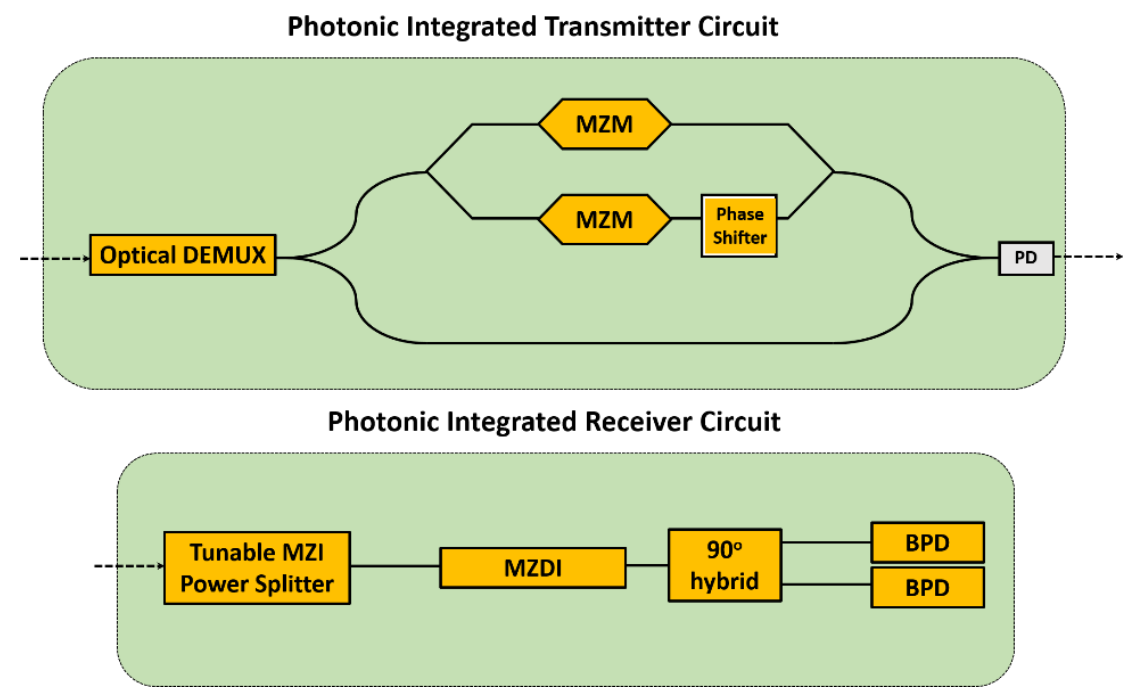

Figure 1.9: Block diagram of the photonic integrated (Up) transmitter (Down) receiver circuits.

The main functional devices to be developed in this thesis are listed below:

- Optical demultiplexer/filter with high extinction ratio (ER) for both ports $(\mathrm{ER} \geq 25 \mathrm{~dB})$ and low insertion losses (IL $\leq 3 \mathrm{~dB})$.

- Optical phase modulator with error free behaviour at $10 \mathrm{Gbit} / \mathrm{s}$ bit-rate, at less.

- Optical phase shifter to achieve a full $\pi$-phase adjustability.

- Tunable Mach-Zehnder power splitter and Mach-Zehnder Delay Interferometer, with propagation losses lower than $5 \mathrm{~dB}$.

- Balanced Photodetectors with a responsivity higher than $0.8 \mathrm{~A} / \mathrm{W}$ and a dark current near to $10 \mathrm{nA}$.

- In addition, a polarization diversity scheme is also studied. Its polarization splitter must present an ER above $20 \mathrm{~dB}$ for both ports, and $\mathrm{IL}<3 \mathrm{~dB}$. The polarization rotator must present a polarization conversion efficiency lower than $-1 \mathrm{~dB}$. 
The introductory chapter was aimed at giving a general description of the benefits of using silicon photonics for Gbps wireless and a state of the art of transceivers developed in the last years in order to meet the increasingly demanding needs' market. Chapter 2 contains an introduction to the PSK modulation formats as well as the key building blocks involved in the $\mathrm{mmW}$ transmitter and the imposed requirements for each device. The work performed in this thesis related with polarization management and DQPSK receiver is described and experimentally demonstrated in Chapter 3. Chapter 4 concludes the thesis giving the most interesting obtained results and an outlook into future. 




\section{Chapter 2}

\section{Transmitter}

\subsection{Introduction}

The aim of this chapter is the design, simulation, fabrication and characterization of the key building blocks involved in the DQPSK millimetre wave photonic transmitter depicted in the figure below:

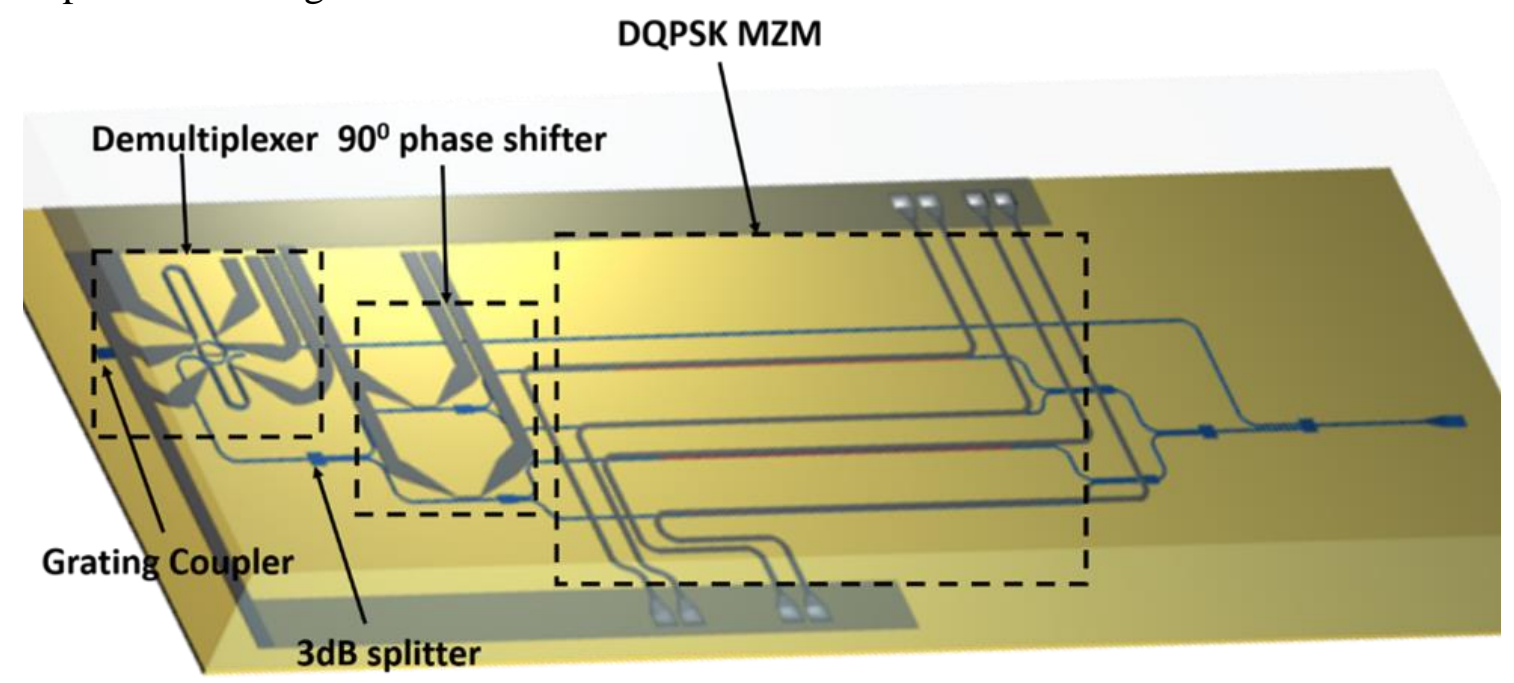

Figure 2.1: Schematic of the integrated millimetre wave transmitter.

These building blocks are:

- Waveguide coupler: A coupling structure for coupling in the light from the laser and out coupling the modulated signal to be transmitted, based on a grating coupler for TE polarization [ROE08].

- DEMUX: The demultiplexer is needed to separate two neighbouring wavelengths with a high extinction ratio. This is discussed in section 2.4. 
- $3 \mathrm{~dB}$ splitter: The device includes $3 \mathrm{~dB}$ splitter for routing the signal through the chip.

- DQPSK MZM: Two DPSK modulators will modulate the electric $10 \mathrm{Gbit} / \mathrm{s}$ signal onto the optical carrier. Each modulator has a $5 \mathrm{Gbit} / \mathrm{s}$ data stream input. The DPSK modulator is discussed in section 2.4.

- $90^{\circ}$ phase shifter: The phase shifter is needed to shift one of the DPSK modulated signal $\pi / 2$ to achieve a DQPSK phase constellation. This is discussed in section 2.3 .

\subsection{Phase-Shift-Keying}

For designing digital optical communication links [IBR07], a wide variety of modulation formats can be chosen. The electric field of the optical carrier can be expressed as:

$$
E(t)=\hat{e} \cdot A e^{-i(\omega t+\varphi)}
$$

Four properties of this optical signal can be modulated: $A$ is the amplitude of the optical field, $\varphi$ is the optical phase, $\omega$ is the optical angular frequency, and $\hat{e}$ is the polarization vector of the laser [WIN06, BIG04]. Each of these parameters can be modulated by an electrical binary baseband signal $q(t)$ :

$$
q(t)=\sum_{i=-\infty}^{+\infty} I_{i} \cdot q\left(t-i T_{b}\right)
$$

with the $i^{\text {th }}$ information coefficient $I \in[0,1]$ and the baseband pulse shape $q(t)$ delayed by multiples of the bit period $T_{b}$. Depending on which parameter of the laser is modulated, the modulation is mainly differentiated as: amplitude shift keying (ASK) [DAI05, HOD02], frequency shift keying (FSK) [IDL04, SAK05], phase shift keying (PSK) [GNA03, BOS04, KIM04, GNA05], or polarization shift keying (PolSK) [EDI01, SID02].

Figure 2.2 shows an electrical binary data stream "101101" used to modulate these four parameters of an optical carrier signal, resulting in the generation of the standard optical digital modulation formats previously presented.

Up to a few years ago, technological difficulties in cost-effective manufacturing devices suitable for high speeds restricted functionalities to the most basic needs, by using intensity modulations of the light emitted by a laser, and detecting it using a simple symbol-by-symbol fixed-threshold. As a result, deployed lightwave systems almost exclusively used on-off keying intensity modulation at the transmitter and square-law photodetection at the receiver; neither coherent demodulation, nor electronic equalization techniques, nor forward error correction (FEC) has been used in the majority of the already installed systems. 


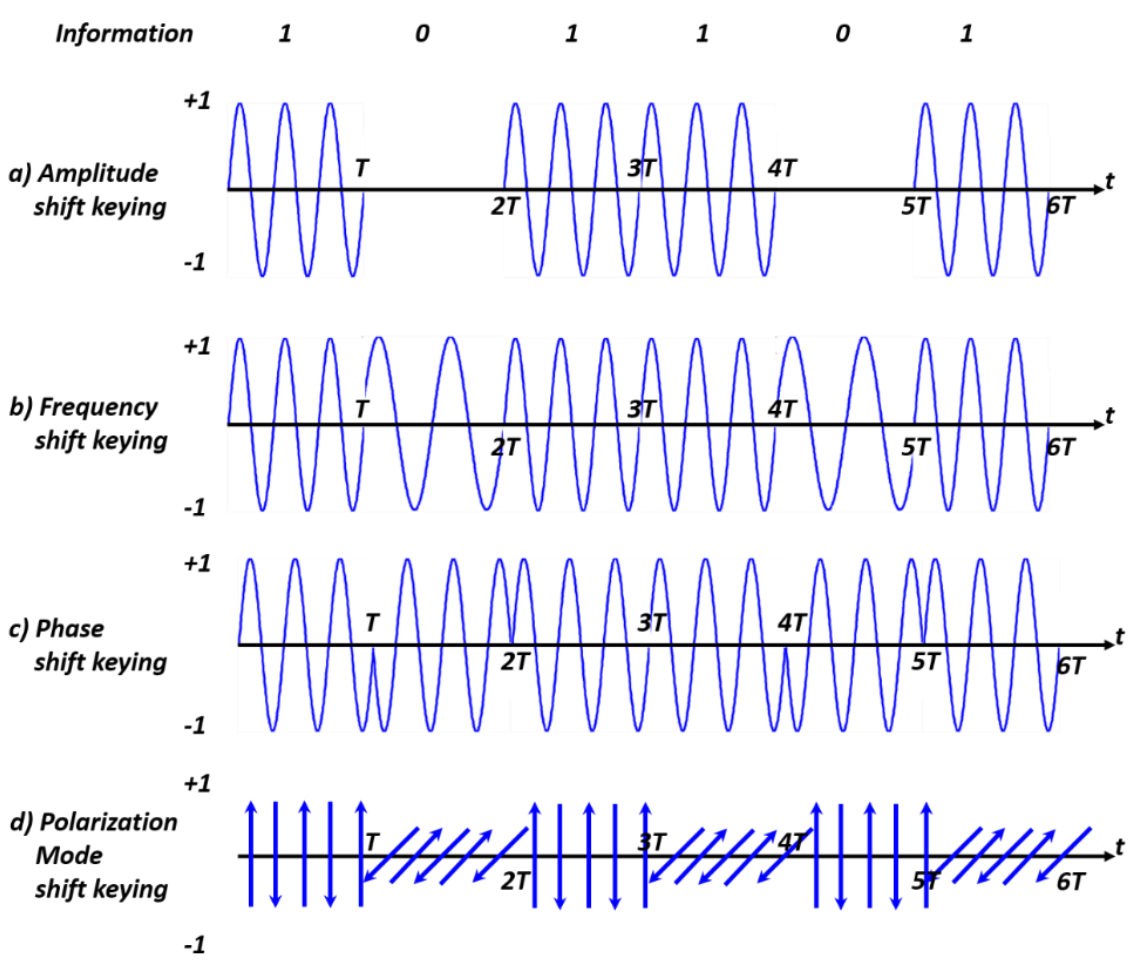

Figure 2.2: The four types of waveform keying with binary coding.

At the turn of the millennium, this situation started to change significantly in such a way that today, advances in high-speed electronics and optoelectronics are not only used to further push per-channel data rates, but also to increase the sophistication of hardware. At $10 \mathrm{Gbit} / \mathrm{s}$ data rates, electronic signal processing ranging from simple feed-forward equalizer (FFE) structures all the way to maximum likelihood sequence estimation (MLSEs) is available today [NIE05, CAS04, FÄB04], and FEC has become a standard feature of $10 \mathrm{Gbit} / \mathrm{s}$ and $40 \mathrm{Gbit} / \mathrm{s}$ commercial optical communication systems [MIZ03]. Controlled signal predistortion at the transmitter is starting to become possible at 10 Gbit/s [McG05], and coherent detection, allowing electronic signal processing to make use of the optical phase information, is experiencing renewed interest [GNA05a, TSU05].

At the same time, systems start to no longer rely exclusively on phase insensitive binary modulation of the optical intensity (OOK), but other modulation formats, such as binary or multilevel phase modulation or partial response formats are being taken into account.

Regarding the physical quantity used to convey digital information, and at the number of symbols used to represent the binary transmit data, the figure below shows a classification of optical modulation formats, where auxiliary modulation features such as pulsed modulation or chirp are also considered. 

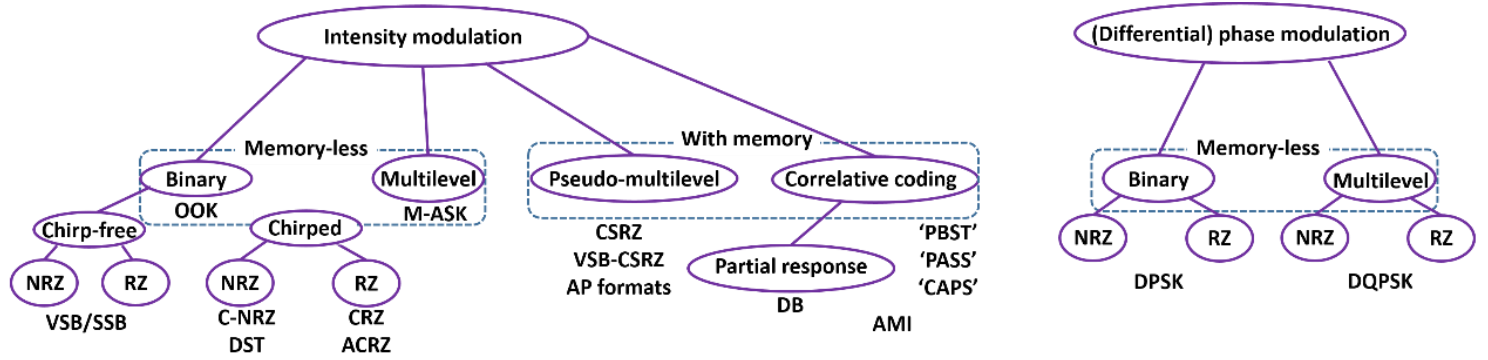

Figure 2.3: Classification of intensity and phase modulation formats for optical communications nowadays [WIN06].

It must be noted that this classification does not require a phase-modulated optical field to be constant-envelope, nor an intensity modulated field to have constant phase. It is the physical quantity used to convey data information that drives the classification. To give some examples: DPSK is a phase-modulated format, regardless of whether it is transmitted constant-envelope or by means of phase modulated optical pulses in the form of RZ-DPSK. On the other hand, CSRZ is an intensity-modulated format, regardless of the fact that the optical field's phase is additionally modulated in order to beneficially influence the spectrum.

While intensity and phase data modulation formats have been widely used in high-speed optical communications, encoding information onto the polarization of light (Pol-SK) has received comparatively little attention [JAC94, BET92, SID02]. This can primarily be attributed to the need for active polarization management at the receiver, necessitated by random polarization changes in optical fiber [LEP00]. However, in recent works, Pol-SK is being considered in order to increase spectral efficiency, either by transmitting two different signals at the same wavelength but in two orthogonal polarizations, or by transmitting adjacent WDM channels in alternating polarizations to reduce coherent WDM crosstalk or nonlinear interactions between the channels [ALF09, SLE11, DOE12b]. Moreover, in the next chapter, a polarization diversity scheme that may be used to implement a polarization division multiplexing is described.

In the early days, PSK did not receive much interest due to its demodulator's complexity. Instead, differential binary PSK (DBPSK, or simply DPSK) has received more interests [HO05]. Since the beginning of optical telecommunications, the most simple modulation format, OOK, has been employed in optical links. With the increases in bit rates, number of optical channels in Dense Wavelength Division Multiplexing (DWDM) configuration, and the augmentation of power in each channel, new modulation formats have been studied in the last years. Today, in order to increase the quality of optical links, tendency is to modify the modulation scheme used to encode information in light signals. Particularly, the DPSK format presents an increased tolerance to non-linear effects in optical fibers, justifying the interest for using this format in optical communications links. In DPSK, the data are first encoded differentially as the differential encoder shown in Figure 2.4. The encoded data are then modulated onto optical carrier using a phase modulator (PM) or Mach-Zehnder modulator (MZM), which externally changes the optical phase from its original phase to a relative $\pi$ phase shift. In response to the driving 
baseband signal [HO05], MZM is preferable to PM due to better chromatic dispersion tolerance.

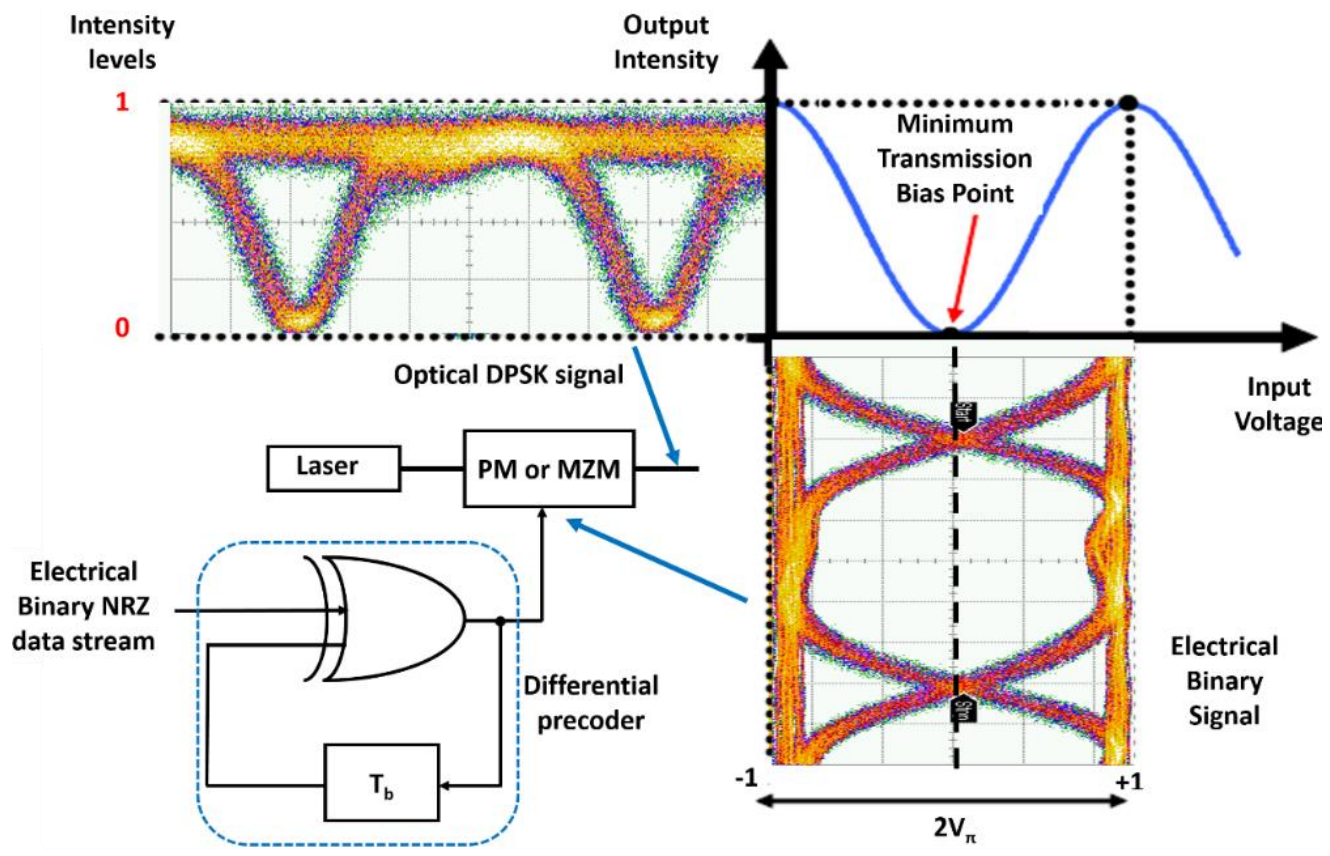

Figure 2.4: Principle of DPSK modulation generation using a MZM.

DPSK encodes information on the binary phase change between adjacent bits: a 1-bit is encoded onto a $\pi$ phase change, whereas a 0-bit is represented by the absence of a phase change. Like OOK, DPSK can be implemented in RZ and NRZ format (see Figure 2.3). The main advantage from using DPSK instead of OOK comes from a 3-dB receiver sensitivity improvement [JAC94], which can be intuitively understood from Figure 2.5, showing that the symbol spacing for DPSK is increased by $\sqrt{2}$ compared to OOK for fixed average optical power [GNA05b].

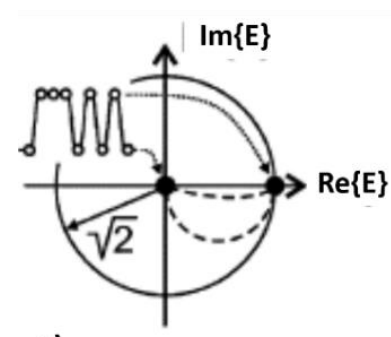

a)

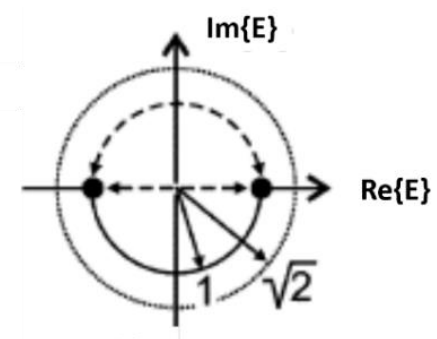

b)

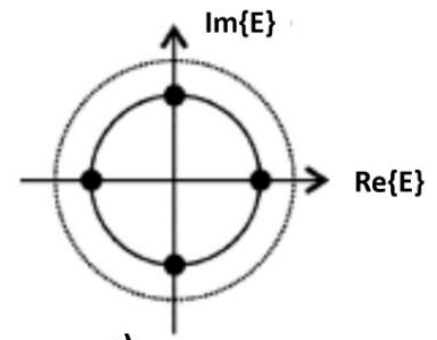

c)

Figure 2.5: Symbol diagrams, normalized to unity average optical power, for a) OOK modulation (dashed lines represent examples of transitions between symbols for chirped formats). b) DPSK modulation (dashed double-arrows represent different phase modulator implementation). c) DQPSK modulation [WIN06].

This increased symbol distance makes DPSK accept a $\sqrt{2}$ larger standard deviation of the optical field noise than OOK for equal bit error rate (BER), which translates into a 3-dB reduction in optical signal to noise ratio (OSNR). An optical DPSK transmitter is shown 
in Figure 2.4, where the data signal is first differentially encoded in order to avoid error propagation that may occur by differential decoding at the receiver [GIT92].

To perform optical phase modulation, one can either, as commented above, use a straightline phase modulator (PM) or an MZM.

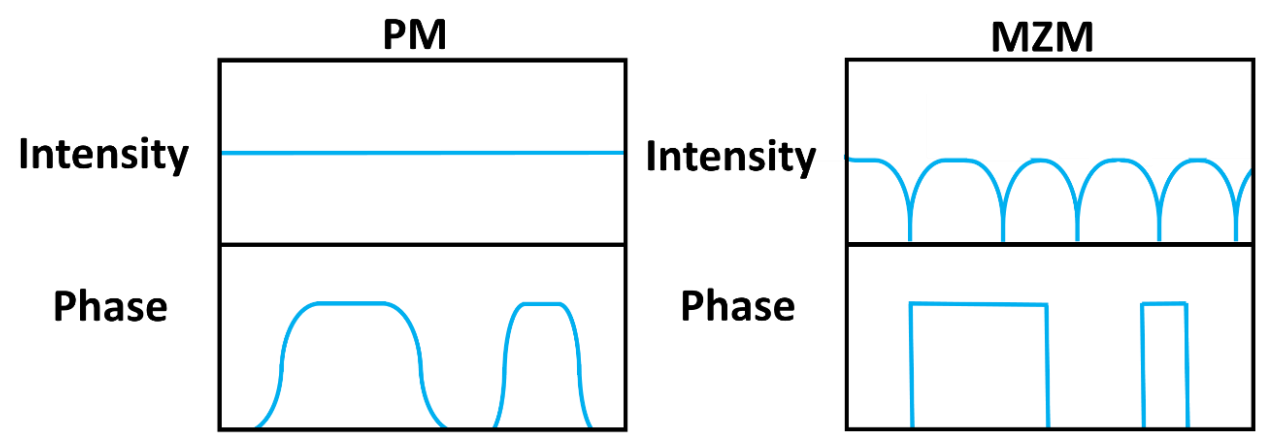

Figure 2.6: Phase modulation can either be achieved using an MZM or by means of a straight-line PM, resulting in different intensity and phase waveforms.

The difference between the two phase modulation schemes is indicated by the dashed double-arrows in Figure 2.5(b): a PM modulates the phase along the unit circle in the complex plane, leaving constant the intensity of the phase-modulated light. This is visualized in Figure 2.6, where the left refers to a PM's intensity and phase waveforms. However, since the optical phase directly follows the electrical drive signal, the speed of phase transitions is limited by the combined bandwidth of driver amplifier and phase modulator, and any overshoot or ringing in the drive waveform manifests itself in phase distortions. An MZM, symmetrically driven around zero transmission, modulates along the real axis through the origin of the complex optical field plane [see Figure 2.5(b)], which always produces exact $\pi$ phase jumps at the expense of residual optical intensity dips at the locations of phase transitions (see MZM waveforms in Figure 2.6). Since exact phase modulation is more important for DPSK than a constant optical intensity, practical DPSK transmitters are most implemented using an MZM as a phase modulator [GNA05b].

DQPSK is a true multilevel modulation format (more than one bit per symbol) that has received appreciable attention in optical communications so far [GRI02a, Gri02B, OHM04a, KIM03, TOK04, OHM04b, CAV04, YOS05]. It transmits the four phase shifts $\{\pi / 4 ; 3 \pi / 4 ;-\pi / 4 ;-3 \pi / 4\}$ at a symbol rate of half the aggregate bitrate. As in the case of DPSK, a DQPSK transmitter is most conveniently implemented by two nested MZMs operating as phase modulators. Figure 2.7 shows the corresponding transmitter setup [GRI02b, GRI01], consisting of a continuously operating laser source, a splitter to divide the light into two paths of equal intensity, two MZMs operating as phase modulators, an optical $\pi / 2$ phase shifter in one of the paths, and a combiner to produce a single output signal. The symbol constellations of the upper and lower paths as well as at the modulator output are also shown, together with the symbol transitions. 


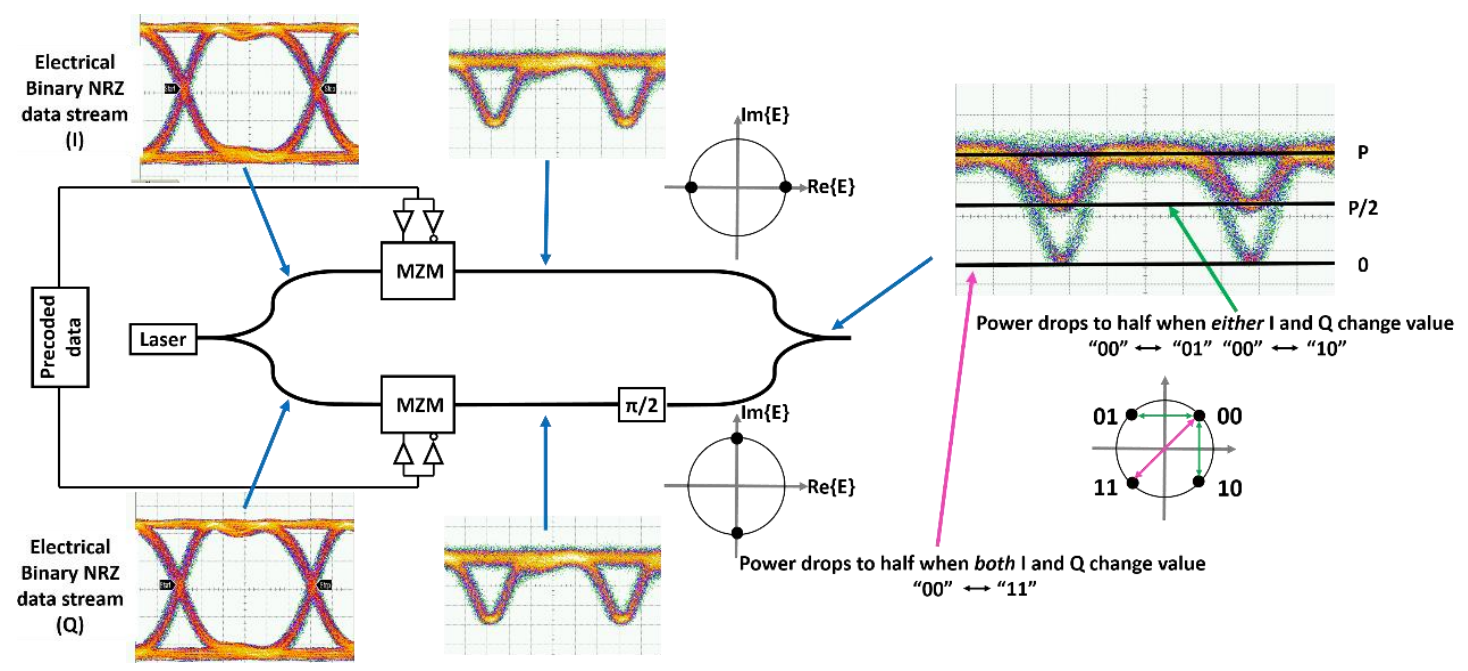

Figure 2.7: Structure of a DQPSK transmitter. Two MZMs are used as phase modulators, and the two separately modulated fields are combined with a $\pi / 2$ phase shift. Constellations and eye diagrams are also shown.

Figure 2.8 shows optical spectra and intensity eye diagrams for NRZ-DPSK and NRZDQPSK, respectively. Note that the shape of the DQPSK optical spectrum is identical to that of DPSK, but the DQPSK spectrum is compressed in frequency by a factor of two due to the halved symbol rate for transmission at fixed bitrate. The compressed spectrum is beneficial for achieving high spectral efficiencies in WDM systems [KRA03, GRI02a], as well as for increased tolerance to chromatic dispersion [GRI02a, WAN04]; the longer symbol duration compared to binary modulation formats makes DQPSK more robust to polarization-mode dispersion.
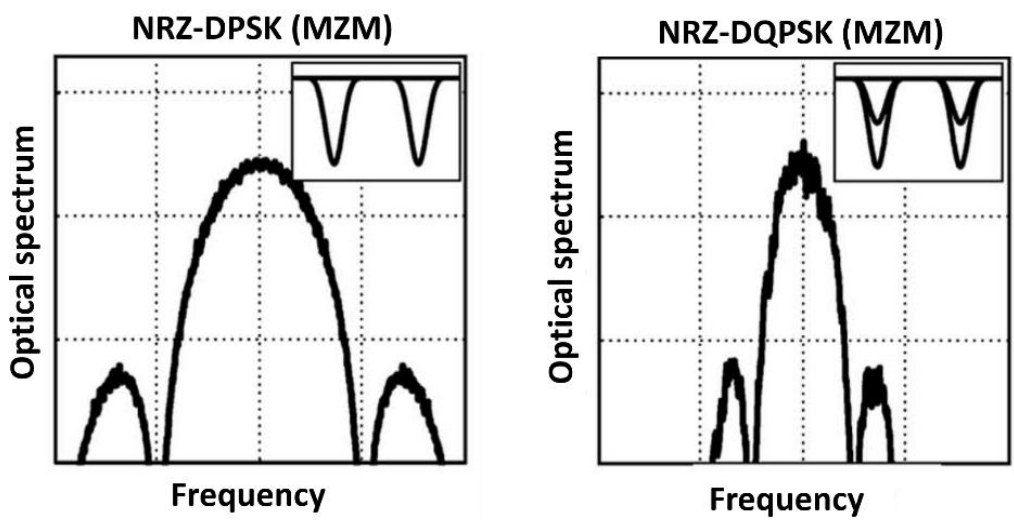

Figure 2.8: Optical spectra and optical intensity eye diagrams for (Left) NRZ-DPSK and (Right) NRZDQPSK [WIN06].

\subsection{Transmitter Description}

The essential element of any photonic transmitter is the modulator. Although many successful demonstrations of amplitude based silicon modulators [SHA08, MIL09] have been reported, phase based encoding modulation has been required due to the exponentially increasing bandwidth requirements. Maximization of the signal to noise 
ratio, minimization of nonlinear effects and maximization of channel efficiency are some additional reasons that make phase modulation preferable over amplitude modulation.

In our case, for the photonic generation of a phase modulated millimetre-wave radio signal, two coherent optical signals separated by a frequency of $60 \mathrm{GHz}$ are generated and injected into the chip, shown in Figure 2.1. These optical carriers are separated using an optical filter/demultiplexer which can be based on different technologies such as MZI, arrayed waveguide grating (AWG), ring resonator, and so on. One of the optical carriers is phase modulated in a DQPSK format using a dual-parallel MZM. Then, the modulated and un-modulated optical carriers are combined and photodetected in order to be wireless transmitted by the antenna.

In QPSK wireless systems, good spectral efficiency is obtained by sending more than one bit of information per hertz of bandwidth. The higher order modulation schemes that achieve more than two states require that the characteristics of the channel to be taken into account. The dominant characteristic of the wireless channel are deep fades resulting from destructive interference of multiple reflections. Fades can be viewed as deep amplitude modulation and so it is difficult to transfer information in the amplitude of a carrier. Consequently phase modulation schemes falling in the class of M-ary phase shift keying (MPSK) are most appropriate in the mobile context. In mobile environments there are just a few modulation formats that have been found acceptable. These all fall in the class of either frequency shift keying (FSK)-like schemes or QPSK. Moreover, and taking into account the intensity waveforms, depicted in Figure 2.6, of the PM and MZM, it becomes clear that MZM should be used for a wireless transmission.

In order to have an error-free DQPSK transmission, a $Q$-factor of about $15.6 \mathrm{~dB}$ must be reached as it can be seen from Figure 2.9 by using the equation shown inset, where erfc denotes the complementary error function:

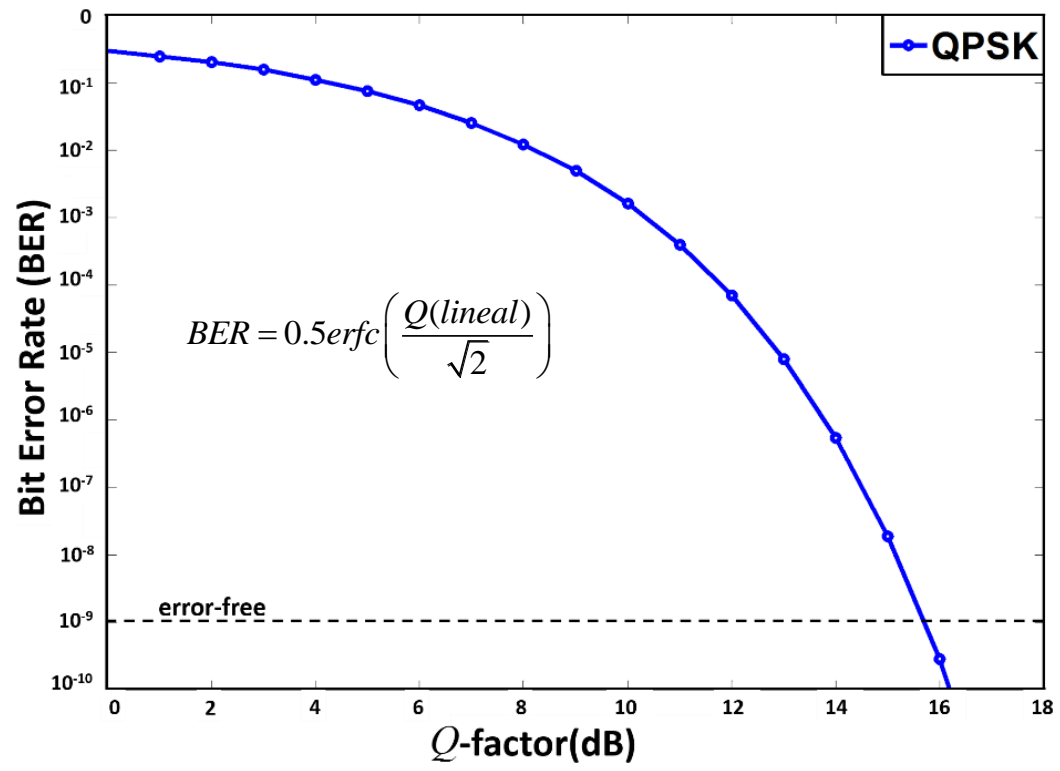

Figure 2.9: Q-factor of the signal as function of the bit error rate (BER), for QPSK modulation. Dashed line indicates $\mathrm{BER}=10^{-9}$, corresponding to a $\mathrm{Q}$-factor of $15.6 \mathrm{~dB}$. 
Different system simulations were carried out in order to set the extinction ratio target of the optical filter to ensure the required $Q$-factor. As it can be seen in the figure below, a value of $29 \mathrm{~dB}$ in the filter rejection ratio is needed for achieving a $Q$-factor of $15.6 \mathrm{~dB}$.

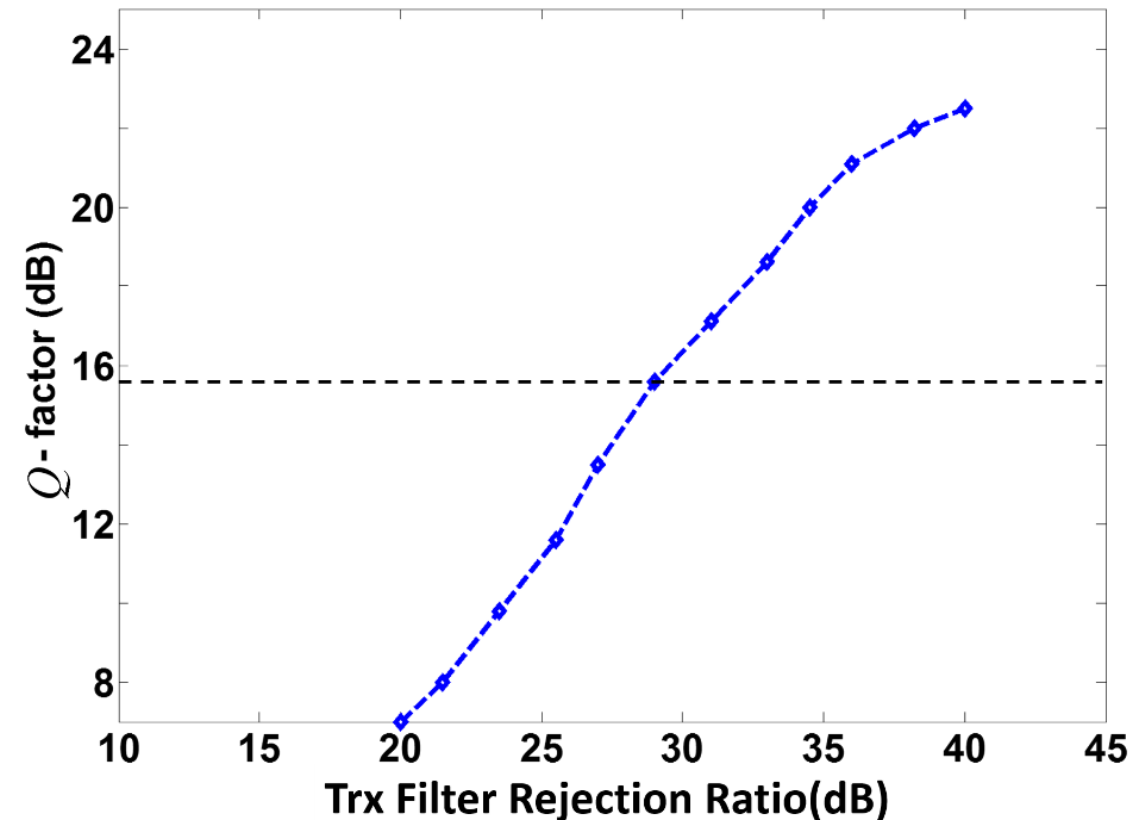

Figure 2.10: Dependence of the transmitter optical filter and the Q-factor of the signal.

Moreover, some simulations were done to set the extinction ratio needed for our system. From the figure below, it is important to notice that the un-modulated port is much more sensible to crosstalk errors than the modulated port, so a higher extinction ratio in the deviated port is needed for a good $Q$-factor.

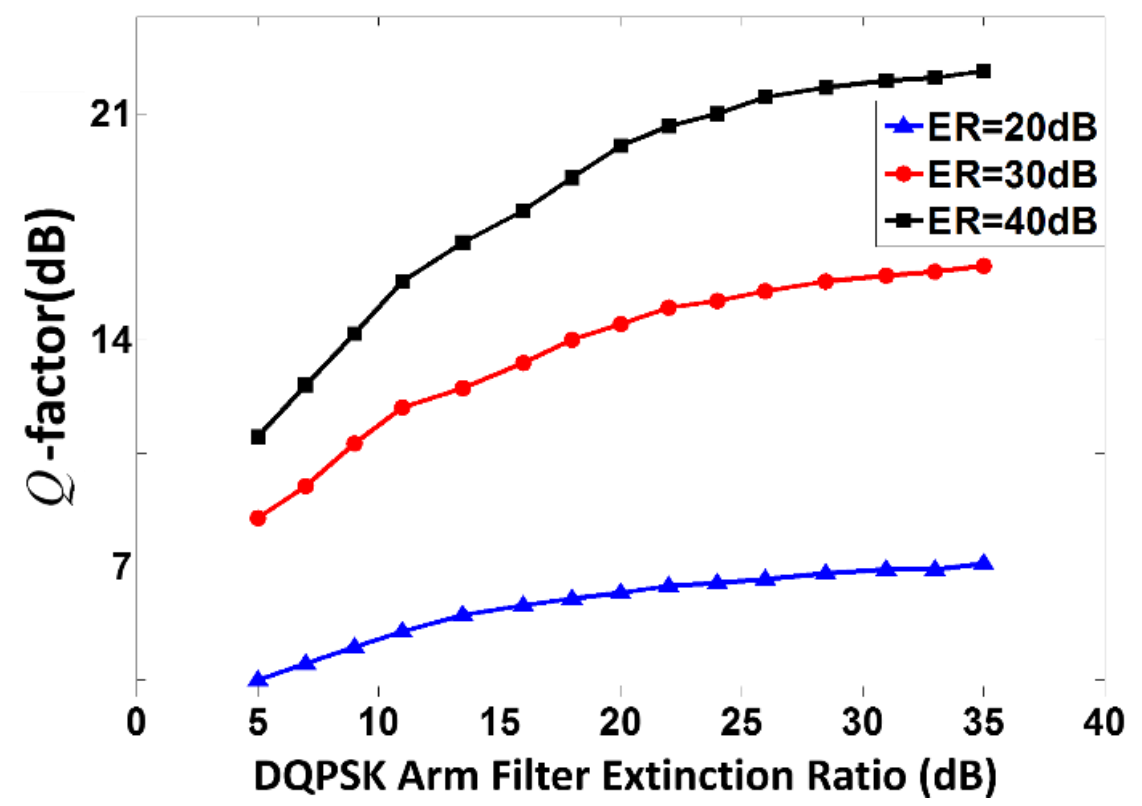

Figure 2.11: Q-factor with respect to the ER achieved in the modulated port (DQPSK Arm) for different values of ER in the un-modulated port ( $\Delta$-blue, $\circ$-red and $\square$-black line). 
In order to cope with fabrication errors and other noise issues, active tuning using microheaters exploiting the thermo-optic effect, will be integrated in the demultiplexer design. From the obtained results shown in the figure above and after a careful selection, the established transmitter targets are:

$$
E R_{\text {modulated_port }} \geq 25 \mathrm{~dB}, E R_{\text {un-modulated_port }} \geq 30 \mathrm{~dB}
$$

Regarding the electro-optic modulator, two DSPK modulators will be included in a nested configuration for DQPSK modulation. This DQPSK generation working in a push-pull configuration using a dual-electrode configuration [DOE12b], with two arms driven by differential data, requires only half of the drive voltages if compared with the singleelectrode configuration, and allows the elimination of the frequency chirp in a broad spectral range [OGA12].

Then, two DPSK modulator structures, which are identical, will form a DQPSK modulator by arranging them in a nested configuration [GNA05], where one of the two modulators has an extra phase shift of $90^{\circ}$ to achieve the DQPSK constellation.

The motivation of the aforementioned push-pull configuration can be clearly seen if the fields in each of the two arms of an unbalanced MZI is analysed. In an ideal case with infinite extinction ratio, the fields in the two arms are expressed as:

$$
\frac{A}{\sqrt{2} e^{-i \pi V_{1} / V_{\pi}}}, \frac{A}{\sqrt{2} e^{-i \pi V_{2} / V_{\pi}}}
$$

where $V_{1}$ and $V_{2}$ are the voltage applied to arm 1 and arm 2 of the Mach-Zehnder, respectively. When adding the two fields using a 3-dB splitter, the sum adds up as expressed in Eq. 2.4:

$$
\frac{A}{2} e^{-i \omega t}\left(e^{-i \pi V_{1} / V_{\pi}}+e^{-i \pi V_{2} / V_{\pi}}\right)
$$

For a single arm driven MZM, the phase and amplitude variation can be calculated by setting $\omega t=0$ and let $V_{1}$ be a constant also equal to 0 , and then vary $V_{2}$ from 0 to $2 V_{\pi}$. Since we are interested in a phase modulation, it is important to show the behaviour of the MZM over the full $2 V_{\pi}$ shift. For uniform amplitude $(A=1)$, the amplitude and phase variation of the MZM can be written as:

$$
\frac{1}{2}+\frac{1}{2} e^{-i \pi V_{2} / V_{\pi}}=\frac{1}{2} e^{\frac{-i \pi V_{2}}{2 V_{\pi}}}\left(e^{\frac{i \pi V_{2}}{2 V_{\pi}}}+e^{\frac{-i \pi V_{2}}{2 V_{\pi}}}\right)=e^{\frac{-i \pi V_{2}}{2 V_{\pi}}} \cdot \cos \frac{\pi V_{2}}{2 V_{\pi}}
$$

with the phase and module of the amplitude as plotted in Figure 2.12.

It can be clearly observed in the figure below that the phase varies linearly from $-\pi / 2$ to $\pi / 2$, with zero amplitude at the singularity point. This instant jump of the phase comes from the change in sign of the cosine function of the amplitude. Anyhow, since the phase is equal at the two points corresponding to maximum amplitude, a single arm MZM cannot be used as a DSPK modulator. 


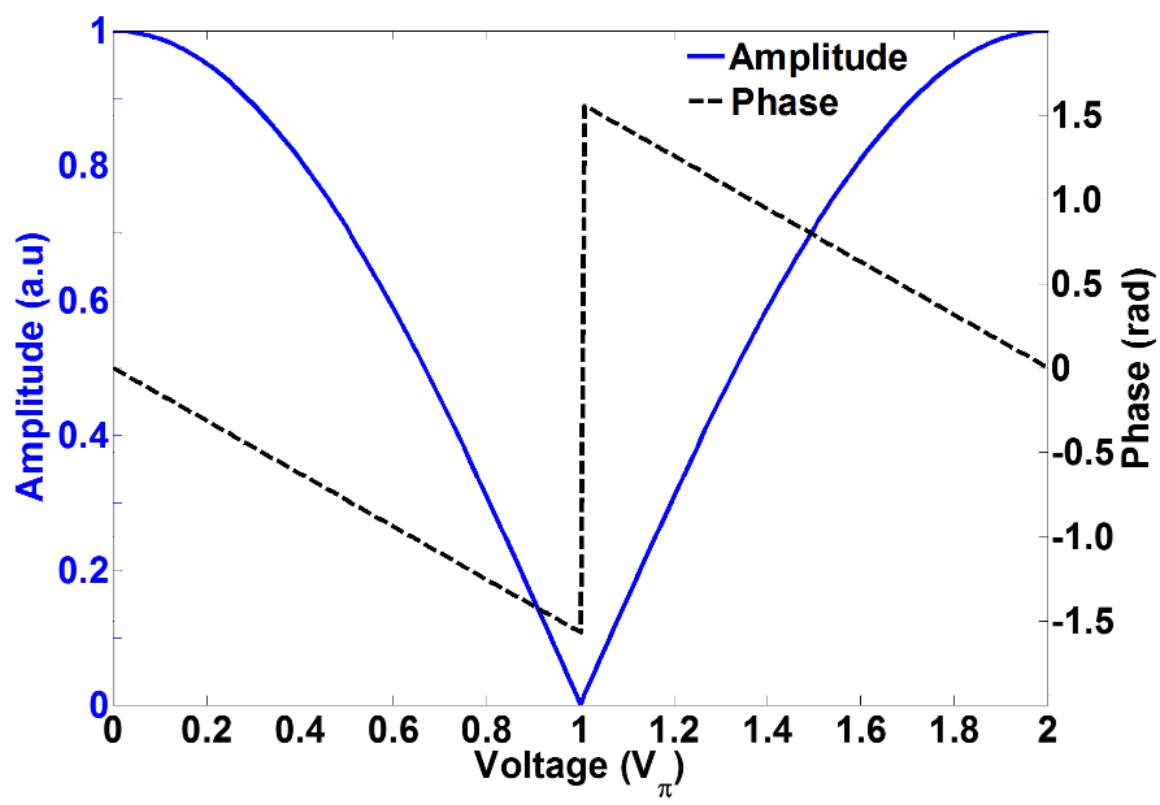

Figure 2.12: Phase (black dashed) and module of the amplitude (blue solid) dependence of a single-armdriven MZM as function of applied voltage to arm2, normalized by $V_{\pi}$.

The linear change of the phase with respect to the applied voltage can be eliminated by a push-pull configuration, where only the instant phase shift at zero amplitude is maintained. This can be demonstrated by setting $V_{1}-V_{0}=V_{0}-V_{2}$, where $V_{0}$ is the bias point. Incorporating this voltage dependency and setting $V_{0}=V_{\pi}, \omega t=0$ and $A=1$, the MZM equation can be now written as:

$$
\frac{1}{2} e^{-i \pi \frac{\left(2 V_{\pi}-V_{2}\right)}{V_{\pi}}}+\frac{1}{2} e^{-i \pi \frac{V_{2}}{2 V_{\pi}}}=\frac{1}{2} e^{-i \pi} \cdot 2 \cos \left(\pi \frac{V_{\pi}-V_{2}}{V_{\pi}}\right)=\cos \left(\pi \frac{V_{2}-V_{\pi}}{V_{\pi}}\right)
$$

It is clearly seen that the expression only shows amplitude dependence and no phase variation. However, the field oscillates from positives to negatives values providing the necessary $\pi$ phase shift for DPSK modulation.

Figure 2.13 shows a graphical representation of the phase and the module of the amplitude variation of Eq. 2.6. As expected, the push-pull configuration only needs $V_{\pi}$ driving voltage to range from one amplitude maximum to the next one due to the two electrodes set-up. The graph also provides a clearer view of how the minimization of the chirp is achieved since there is no linear change in the phase. It must be commented that in a more realistic case, we will not experience an instantaneous phase shift due to the limited extinction ratio. 


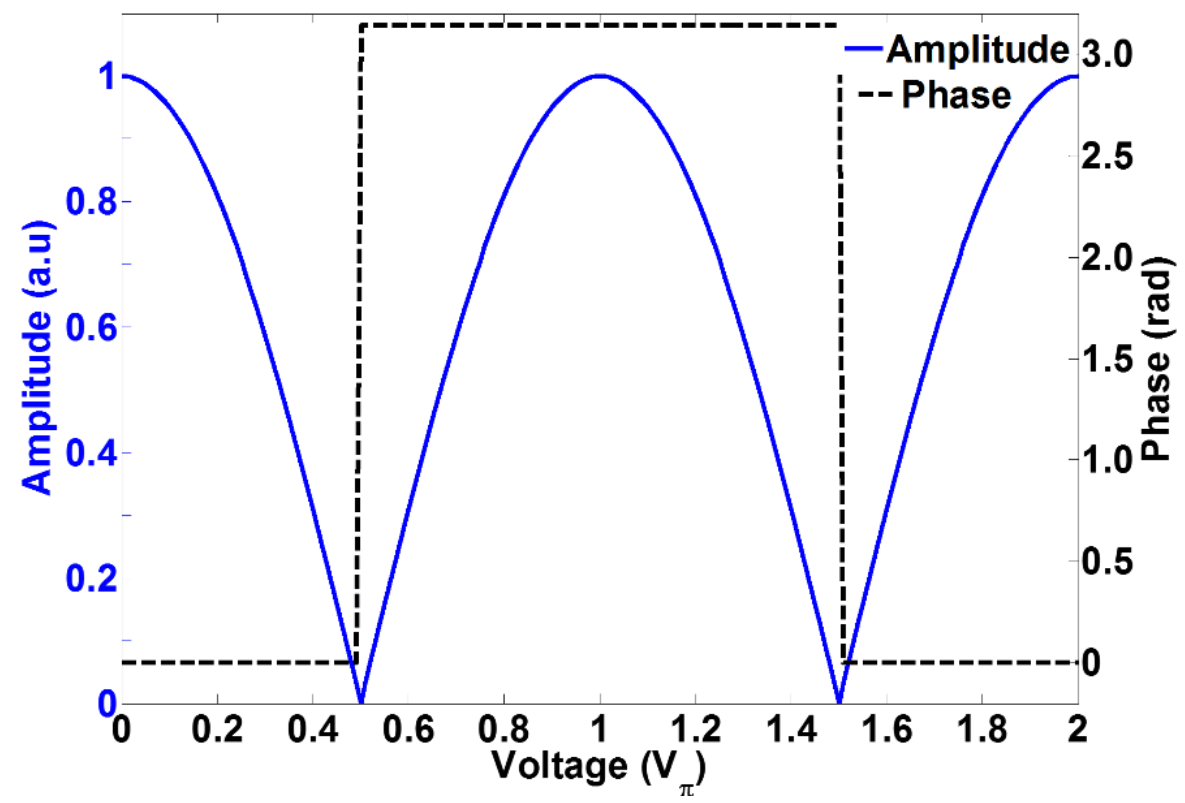

Figure 2.13: Phase (black dashed) and amplitude module (blue solid) variation of a MZM in push-pull configuration.

The next element, the $90^{\circ}$ phase shifter, is incorporated in the DQPSK MZM and will be realized using a delay line. The length of this delay line depends on the guided mode in the waveguide. Figure 2.14 shows an example of a strip waveguide of silicon, surrounded by silica, with an estimated effective phase propagation index of about $n_{\text {eff }}=2.4$. This waveguide presents a delay coefficient of $0.56 \mathrm{deg} / \mathrm{nm}$, meaning that a full $90^{\circ}$ phase shift will be achieved in a $161 \mathrm{~nm}$ delay line.

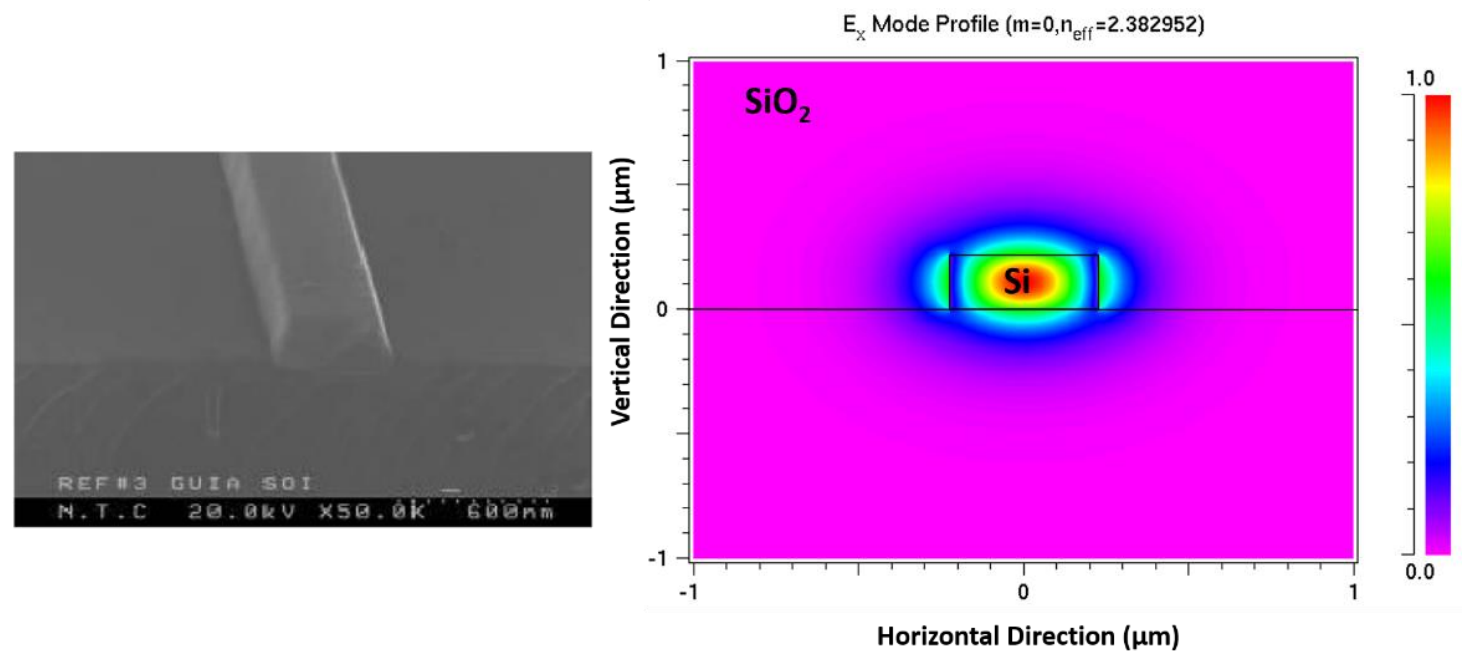

Figure 2.14: SOI waveguide with an estimated $n_{\text {eff }}$ of 2.38. (Left) SEM image and (Right) a mode profile calculation.

In order to cope with fabrication errors and environmental temperature dependency, a control element has to be included, and the selected implementation is by incorporating micro-heaters. This heating element can be implemented by a thin metallic wired positioned in close proximity to the dielectric waveguide [SHE08]. The higher resistance 
of the wire will heat up the surrounding material and modify the dielectric index of the waveguide.

As commented in Chapter 1, this work is framed in the European project HELIOS [HEL], and the obtained performance requirements for each building block are described in the following table.

TABLE 2-1

BUILDING BLOCKS AND HELIOS PERFORMANCE REQUIREMENTS FOR MILLIMETRE WAVE TRANSMITTER

\begin{tabular}{|c|c|c|}
\hline Device & Description & $\begin{array}{l}\text { Performance } \\
\text { Requirements }\end{array}$ \\
\hline Demultiplexer/Filter & $\begin{array}{l}\text { Device needed in order to } \\
\text { separate two optical carriers } \\
\text { separated } 60 \mathrm{GHz} \text { at } 1550 \\
\text { nm with high extinction } \\
\text { ratio. }\end{array}$ & $\begin{array}{l}\text { ERmodulated_port } \geq \mathbf{2 5 d B} \\
\text { ERun-modulated_port } \geq \mathbf{3 0 d B} \\
\text { IL } \leq \mathbf{3 d B}\end{array}$ \\
\hline DPSK MZM & $\begin{array}{l}\text { Two DPSK modulators will } \\
\text { be included in a nested } \\
\text { Mach Zehnder for DQPSK } \\
\text { modulation, and will } \\
\text { modulate the electrical } 10 \\
\text { Gbit/s signal onto the } \\
\text { optical carrier. Each } \\
\text { modulator has a } 5 \mathrm{Gbit} / \mathrm{s} \\
\text { data stream input. }\end{array}$ & $\begin{array}{l}\text { ER as high as possible } \\
\text { Push-pull configuration }\end{array}$ \\
\hline $90^{\circ}$ Phase Shifter & $\begin{array}{l}\text { The phase shifter will be } \\
\text { implemented as an active } \\
\text { delay line with a micro- } \\
\text { heater control element, in } \\
\text { order to shift one of the } \\
\text { DPSK modulated signal } \pi / 2 \\
\text { to achieve a DQPSK phase } \\
\text { constellation }\end{array}$ & $\begin{array}{l}\text { Active DC tuning to } \\
\text { achieve a full } \pi \text {-phase } \\
\text { adjustability. }\end{array}$ \\
\hline
\end{tabular}




\subsection{Optical Filter/Demultiplexer}

Ring resonators are one of the most suitable and versatile structures to achieve very compact filters with high performance. Microring filters have been extensively analysed to implement multiplexers/demultiplexers for multiple channel transmission in wavelength division multiplexing (WDM) systems and on chip optical interconnects [XIA07a, XIA07b, ZHE10]. Here in HELIOS, a microring demultiplexer filter is especially developed as a building block to be part of a photonic millimetre wireless transmitter. The performance requirements, as shown in Table 2-1 are:

- Insertion losses below $3 \mathrm{~dB}$

- Extinction ratios of $25 \mathrm{~dB}$ and $30 \mathrm{~dB}$ for the modulated and un-modulated ports, respectively.

First, a brief theoretical introduction to ring resonators and key parameters will be presented in the next subsection.

\subsubsection{Design and fabrication}

The basic configuration, which consists of unidirectional coupling between a ring resonator (resonant at a wavelength $\lambda_{\mathrm{i}}$ ) with radius $\mathrm{R}$ and a waveguide, is shown in Figure 2.15, based on [YAR02, RAB07].

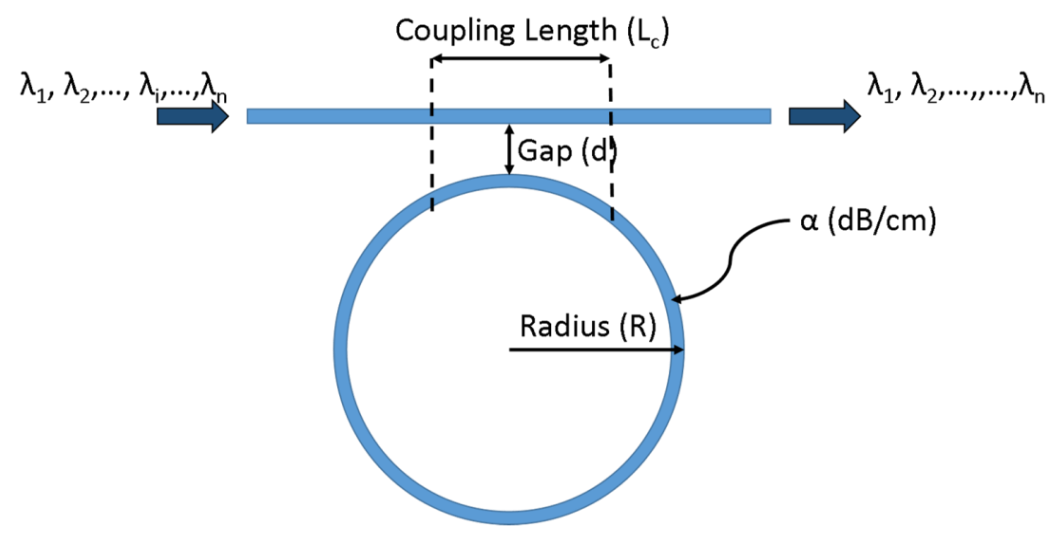

Figure 2.15: Design parameters for a ring resonator.

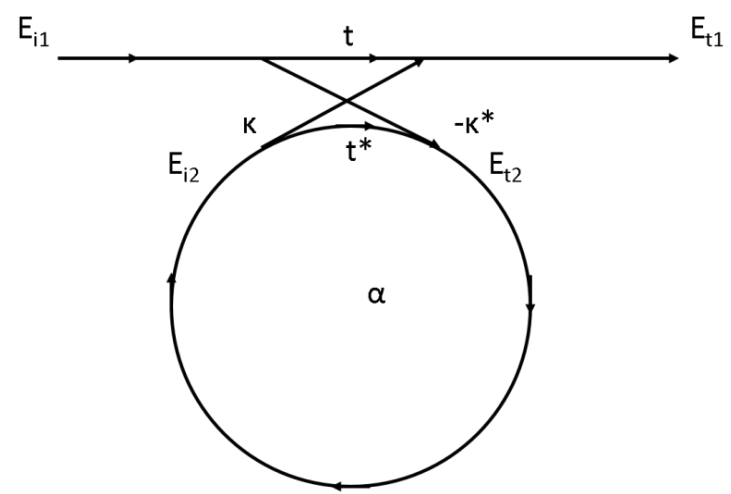

Figure 2.16: Model of a single ring resonator with one waveguide. 
Defining that a single unidirectional mode of the resonator is excited, the coupling is lossless, single polarization is considered, and taking into account that the different kinds of losses in the ring resonator are incorporated in the attenuation constant, the interaction can be described by the matrix relation:

$$
\left(\begin{array}{l}
E_{t 1} \\
E_{t 2}
\end{array}\right)=\left(\begin{array}{cc}
t & \kappa \\
-\kappa^{*} & t^{*}
\end{array}\right)\left(\begin{array}{l}
E_{i 1} \\
E_{i 2}
\end{array}\right)
$$

The complex mode amplitudes $E$ are normalized, so that their squared magnitude corresponds to the modal power. The coupler parameters $t$ and $\kappa$ depend on the specific coupling mechanism used. The $*$ denotes the conjugated complex value of $t$ and $\kappa$, respectively. The matrix is symmetric because the networks under consideration are reciprocal. Therefore:

$$
\left|\kappa^{2}\right|+\left|t^{2}\right|=1
$$

In order to simplify the model, $E_{i 1}$ is chosen to be equal to 1 . Then the round trip in the ring is given by:

$$
E_{i 2}=\alpha \cdot e^{j \theta} \cdot E_{t 2}
$$

where $\alpha$ is the loss coefficient of the ring (zero loss: $\alpha=1$ ) and $\theta=\omega L / c$, being $L$ the length of the ring which is given by $L=2 \pi R$, where $R$ is the radius of the ring measured from the center of the ring to the center of the waveguide, $c$ the phase velocity of the ring mode $\left(c=c_{0} / n_{\text {eff }}\right)$ and the fixed angular frequency $\omega=k \cdot c_{0}, c_{0}$ refers to the vacuum speed of light. The vacuum wavenumber $k$ is related to the wavelength $\lambda$ through: $k=2 \pi / \lambda$. Using the vacuum wavenumber, the effective refractive index $n_{\text {eff }}$ can be introduced easily into the ring coupling relations by:

$$
\beta=k \cdot n_{\text {eff }}=\frac{2 \pi \cdot n_{\text {eff }}}{\lambda}
$$

where $\beta$ is the propagation constant. This leads to

$$
\theta=\frac{\omega L}{c}=\frac{k c_{0} L}{c}=k \cdot n_{\text {eff }} \cdot 2 \pi R=\frac{2 \pi \cdot n_{\text {eff }} \cdot 2 \pi R}{\lambda}=4 \pi^{2} n_{\text {eff }} \frac{R}{\lambda}
$$

From Eq. 2.7 and Eq. 2.9 we obtain:

$$
\begin{aligned}
& E_{t 1}=\frac{-\alpha+t \cdot e^{-j \theta}}{-\alpha t^{*}+e^{-j \theta}} \\
& E_{i 2}=\frac{-\alpha \kappa^{*}}{-\alpha t^{*}+e^{-j \theta}}
\end{aligned}
$$




$$
E_{t 2}=\frac{-\kappa^{*}}{1-\alpha t^{*} e^{j \theta}}
$$

This leads to the transmission power $P_{t 1}$ in the output waveguide, which is:

$$
P_{t 1}=\left|E_{t}\right|^{2}=\frac{\alpha^{2}+|t|^{2}-2 \alpha|t| \cos \left(\theta+\varphi_{t}\right)}{1+\alpha^{2}|t|^{2}-2 \alpha|t| \cos \left(\theta+\varphi_{t}\right)}
$$

where $t=|t| \exp \left(j \varphi_{t}\right),|t|$ representing the coupling losses and $\varphi_{t}$ the phase of the coupler.

The circulating power $P_{i 2}$ in the ring is given by:

$$
P_{i 2}=\left|E_{i 2}\right|^{2}=\frac{\alpha^{2}\left(1-|t|^{2}\right)}{1+\alpha^{2}|t|^{2}-2 \alpha|t| \cos \left(\theta+\varphi_{t}\right)}
$$

On resonance, $\left(\theta+\varphi_{t}\right)=2 \pi m$, where $m$ is an integer, we obtain that:

$$
P_{t 1}=\left|E_{t}\right|^{2}=\frac{(\alpha-|t|)^{2}}{(1-\alpha|t|)^{2}}
$$

and

$$
P_{i 2}=\left|E_{i 2}\right|^{2}=\frac{\alpha^{2}\left(1-|t|^{2}\right)}{(1-\alpha|t|)^{2}}
$$

A special case happens when $\alpha=|t|$ in Eq. 2.17, when the internal losses are equal to the coupling losses, so the transmitted power equals zero, which is known in literature as critical coupling, due to destructive interference. By using the above equations, the behaviour of a basic ring resonator filter configuration consisting of only one waveguide and one ring can be taken. This model can be extended to suit the requirement of various types of ring resonator configurations.

Ring resonator filters can be described by some figures of merit which will be explained in the next paragraphs. The first figure is free spectral range (FSR), which represents the distance between resonance peaks. By using the propagation constant from Eq. 2.10, neglecting the wavelength dependency of the effective refractive index, a simple approximation can be obtained for the FSR

$$
\frac{\partial \beta}{\partial \lambda}=-\frac{\beta}{\lambda}+k \frac{\partial n_{e f f}}{\partial \lambda} \approx-\frac{\beta}{\lambda}
$$

which leads to the FSR $=\Delta \lambda$ expressed in Eq. 2.20, which is the difference between the vacuum wavelengths corresponding to two resonant conditions. 


$$
F S R=\Delta \lambda=-\frac{2 \pi}{L}\left(\frac{\partial \beta}{\partial \lambda}\right)^{-1} \approx \frac{\lambda^{2}}{n_{e f f} L}
$$

Note that the equation above is for the resonant condition next to a resonance found for the used propagation constant. If the wavelength dependence of the effective index is not neglected, we will obtain a modified version of Eq. 2.19:

$$
\frac{\partial \beta}{\partial \lambda}=-\frac{k}{\lambda} n_{g}
$$

where $n_{g}$ is the group refractive index, which is defined as:

$$
n_{g}=n_{e f f}-\lambda \frac{\partial n_{e f f}}{\partial \lambda}
$$

The group refractive index can be used instead of the effective index in order to obtain accurate values when avoiding approximation is important, which is the case of silicon. The modified FSR $\Delta \lambda$ is then given by:

$$
F S R=\Delta \lambda=\frac{\lambda^{2}}{n_{g} L}
$$

The next parameter of importance is the resonance width which is defined as the full width at half maximum (FWHM) or $3 \mathrm{~dB}$ bandwidth $(2 \delta \lambda)$ of the resonance lineshape.

Using Eq. 2.11 and Eq. 2.19 and using the real part of the series expansion of the Euler formula, after some operations, we obtain that:

$$
2 \delta \lambda=\frac{\lambda^{2}}{\pi L n_{g}} \frac{1-t^{2}}{t}
$$

The expression which is commonly used can be obtained by assuming critical coupling is:

$$
F W H M=2 \delta \lambda=\frac{k^{2} \lambda^{2}}{\pi L n_{g}}
$$

The finesse $F$ of the ring resonator filter, which is defined as the ratio of the FSR and the width of a resonance for a specific wavelength (FWHM) is another parameter which can now be directly calculated from the parameters defined previously is:

$$
F=\frac{F S R}{F W H M}=\frac{\Delta \lambda}{2 \delta \lambda}=\pi \frac{t}{1-t^{2}} \simeq \frac{\pi}{k^{2}}
$$


A parameter which is closely related to the finesse is the quality factor $Q$ of a resonator, which is a measure of the sharpness of the resonance. It is defined as the ratio of the operation wavelength and the resonance width:

$$
Q=\frac{\lambda}{\delta \lambda}=\pi \frac{n_{g} L}{\lambda} \frac{t}{1-t^{2}}=\frac{n_{g} L}{\lambda} F
$$

One key figure of merit of resonators is their bandwidth, which is inversely proportional to the quality factor $Q$ defined in Eq. 2.27. While a narrow bandwidth (high $Q$ ) is suitable for enhancing light-matter interactions such as lasing [REI06] and sensing [ARM06], a wide bandwidth (low $Q$ ), on the other hand, allows for optical signals of a broader spectrum and higher speed [LEE06] and is more tolerant to variations in the environment. Several applications require resonators with a tunable bandwidth or $Q$. One example is a reconfigurable channel selector for wavelength division multiplexing systems where the bandwidth of an add-drop filter can be tuned to accommodate one or several channels within one resonance and switch them simultaneously [PAW96]. The bandwidth of a resonator is determined by its intrinsic loss and coupling with the input and/or output ports.

Previously, the simplest ring resonator configuration was presented (see Figure 2.15). Now, an add-drop microring configuration is considered and shown in Figure 2.17(a). We will assume that the round-trip intrinsic power loss is $\alpha(<<1)$ and that the power coupling with the input and output waveguides is $\kappa$ and $\kappa^{\prime}$ respectively. After each round trip the optical power circulating in the ring is reduced [KOO06] by:

$$
G \approx(1-\alpha)(1-\kappa)\left(1-\kappa^{\prime}\right)
$$

The FWHM bandwidth can be then written as:

$$
\Delta \lambda=\frac{\lambda_{0}^{2}}{2 \pi n_{g} L} \ln \left(\frac{1}{G}\right)
$$

where $\lambda_{0}$ and $n_{g}$ are the resonant wavelength and group index, respectively, $L=2 \pi R$ is the physical length of the ring, and In the natural logarithm. Any tuning of the loss or coupling will result in a change in the bandwidth [YAO07]. However, a high extinction ratio can only be obtained when critical coupling occurs, i.e., when $\kappa=\kappa^{\prime}+\alpha$, in which case individual tuning leads to a degraded extinction ratio, but if $\kappa$ and $\kappa^{\prime}$ are changed accordingly so that $\kappa=\kappa^{\prime}+\alpha$ remains valid, tunability of the bandwidth will be feasible while a high extinction ratio is maintained. The coupling of the resonator with the input and/or output ports can be changed dynamically with tunable interferometric couplers [POP07] as shown in Figure 2.17(b). 
a)

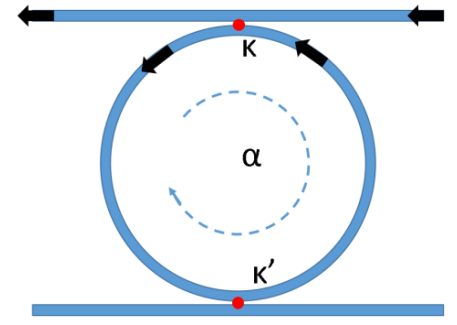

b)

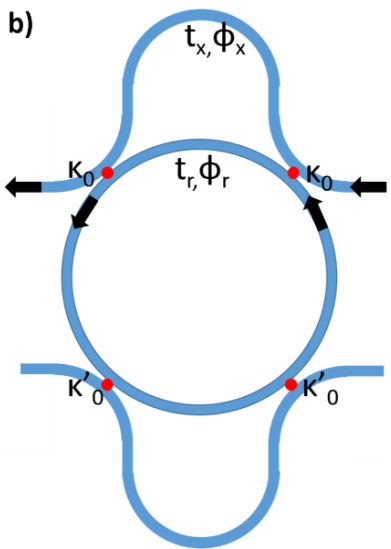

Figure 2.17: Schematic of an add-drop microring resonator with (a) straight couplers and (b) interferometric couplers

From Figure 2.17(b), we assume that the power coupling at each point is $\kappa_{0}$ and that the transmission and phase of the interferometric and ring arms are $t_{x}, t_{r}$ (close to 1) and $\phi_{x}$ , $\phi_{r}$, respectively. Then, the effective coupling from the waveguide to the ring can be described by Eq. 2.30:

$$
\kappa=\kappa_{0}\left(1-\kappa_{0}\right)\left(t_{x}+t_{r}-2 \sqrt{t_{x} t_{r}} \cos \left(\phi_{x}-\phi_{r}\right)\right)
$$

From the equation above, we can see that if the relative phase $\Delta \phi=\left(\phi_{x}-\phi_{r}\right)$ experiments a change (by thermo-optical effect, for example) from 0 to $\pi$, the effective coupling will range between 0 and $4 \cdot \kappa_{0}\left(1-\kappa_{0}\right)$ [CHE07].

The resonances in this structure, contrary to resonances in standard ring resonators, exhibit distinctively different bandwidths and extinction ratios that are oscillating with wavelength. These oscillations originate from the wavelength dependence of $\kappa$ since:

$$
\Delta \phi=\frac{2 \pi \cdot n_{e f f} \cdot\left(L_{x}-L_{r}\right)}{\lambda}
$$

where $n_{\text {eff }}$ is the effective index and $L_{x}, L_{r}$ are the physical lengths of the two interfering arms. Regarding the phase/wavelength tuning, the refractive index in the ring resonator can be modified by using the thermo-optic effect or by electro-optical effect. So, by tuning the refractive index of the silicon and hence the effective index of the mode, a single wavelength can be switched between the output ports. A shift of the effective index, $\Delta n_{\text {eff }}$ , causes a shift of the resonance wavelength [NAW06] as depicted in Figure 2.18 and it is given by the equation below:

$$
\Delta \lambda=\lambda_{0} \frac{\Delta n_{\text {eff }}}{n_{g}}
$$




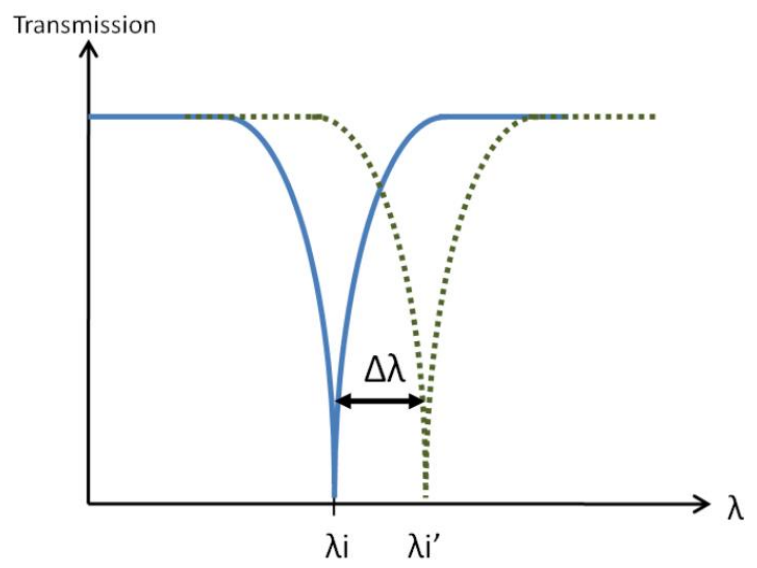

Figure 2.18: Shift of the resonant wavelength due to a shift of the effective index.

As described in the previous section, the chip will route two signals separated $60 \mathrm{GHz}$, equalling $0.48 \mathrm{~nm} @ \lambda=1550 \mathrm{~nm}$, through the different building blocks. The two wavelengths must be separated in the demultiplexer, and the splitting of the deviated and modulated signal is crucial because a very good isolation of the signal is needed in order to achieve error-free transmission. Therefore a ring resonator add-drop filter was selected for this building block. In order to cope with heat, fabrication errors and other noise issues, various micro-heaters were included in the design of our filter. By using a set of specific microheaters, the ER as well as the Q-value and selectivity can be controlled.

Two configurations were considered in this study. The first one is shown in Figure 2.19(a), which is based on a double coupled through and single coupled drop RR. Using this double coupling structure for the through coupling, it is possible to control the ER of the through port. Consequently, the drop port response will also change according to the changes in the through port. By tuning the coupling, a maximized ER in the through port can be achieved when the coupling coefficient is exactly identical to the losses in the ring, and therefore, this configuration will allows us to actively modify the ER in order to achieve the set specification.

The phase of the ring resonator is also tuned using a microheater located exactly on top of the ring waveguide. In order to avoid any change of the coupling coefficients to the ring, the micro-heater is only present outside the coupling regions of the ring as it can be seen in Figure 2.19(b).

Since the coupling to the drop port is fixed in this first configuration, we will not be able to control the $Q$-value of the filter when tuned, and therefore its bandwidth. 
a)

b)

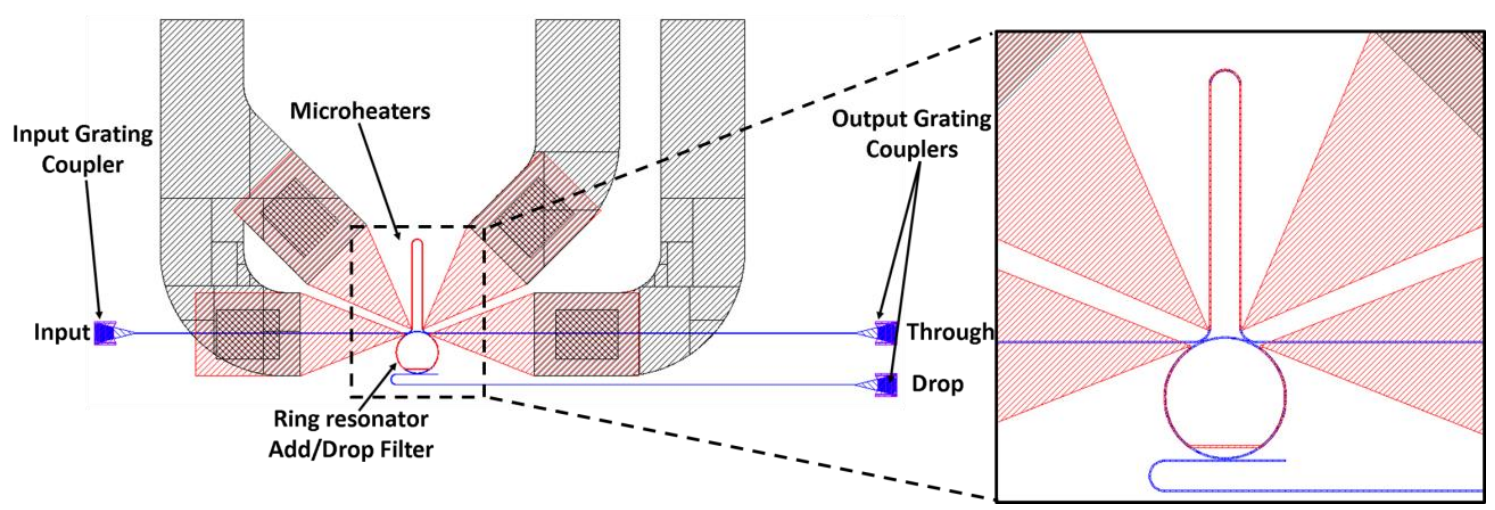

Figure 2.19: (a) Add-drop filter using a single tuned ring resonator where only the coupling through port is tuned as well as the phase. The red striped layer corresponds to the microheater metallization, the black striped corresponds to the pads and the blue to the optical waveguides. (b) A close-up of the single-tuned RR filter.

In order to also control the $Q$-value and ER of the drop port, for a correct $60 \mathrm{GHz}$ separation, a second configuration has been included in the study. As seen in Figure 2.20, the coupling coefficient of both the through and drop port can be controlled.

a)

b)

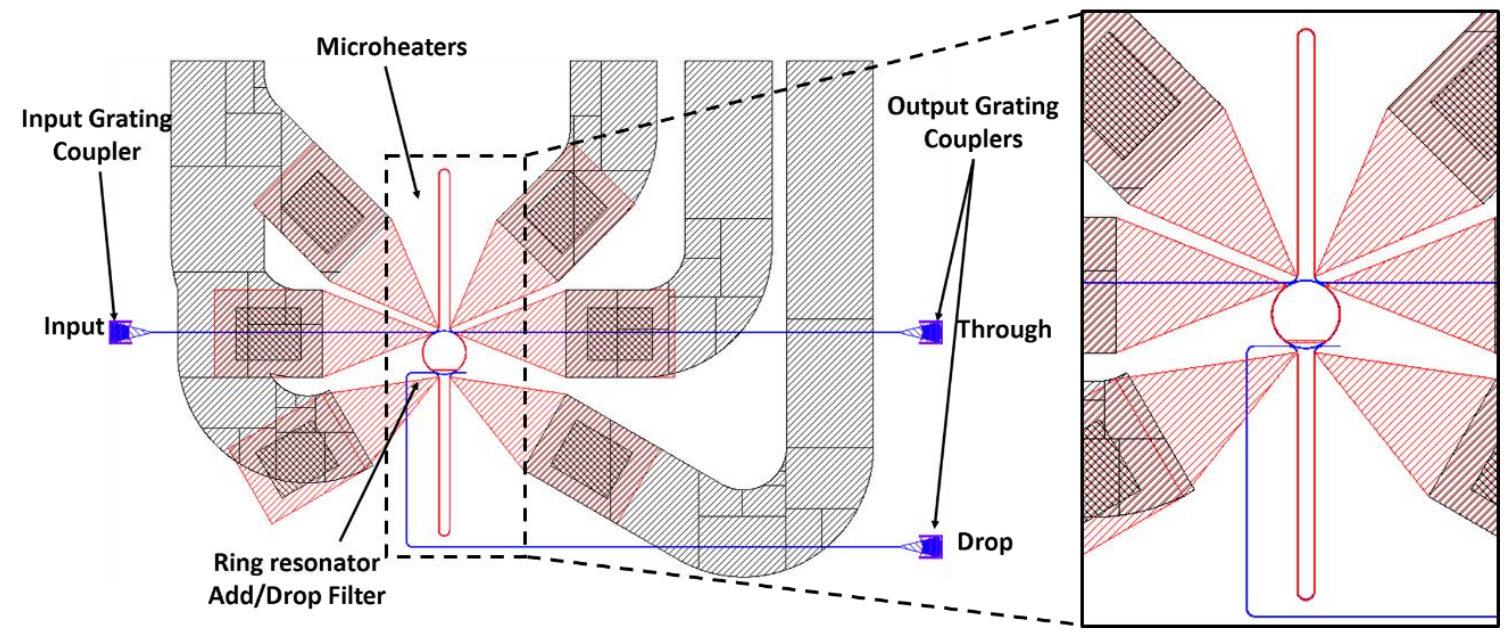

Figure 2.20: (a) Add-drop filter using a double tuned ring resonator where both through and drop are tuned, as well as the phase. The red striped layer corresponds to the microheater metallization, the black striped corresponds to the pads and the blue to the optical waveguides. (b) A close-up of the double-tuned ring resonator filter.

By using transfer matrix formalism [POO04], the spectral response of the whole structure of the double-tuned ring resonator configuration has been modelled. Figure 2.21(Left) shows the effect of tuning the input coupling $\kappa$ with the heater corresponding to the through coupling. The output coupling $\kappa^{\prime}$ (drop coupling) is maintained constant. The device used here has $L_{x}=255.71 \mu \mathrm{m}$, and the resonance measured is at a wavelength close to $\lambda=1550.4 \mathrm{~nm}$. Starting from the "overcoupled" condition (I) where the extinction ratio is of about $40 \mathrm{~dB}$, the coupling first rises to a maximum (II) and then gradually moves 
across the "critical coupling" level (III), reaching a the maximum ER achievable until the minimum (IV), where the resonance is almost switched off. Then, the coupling crosses the critical coupling level again (V) presenting again the maximum ER value, and rises to an "overcoupled" condition. Figure 2.21 (Right) shows the corresponding spectra. It can be seen that the resonance can be completely switched off and on between state (IV) and state (V).

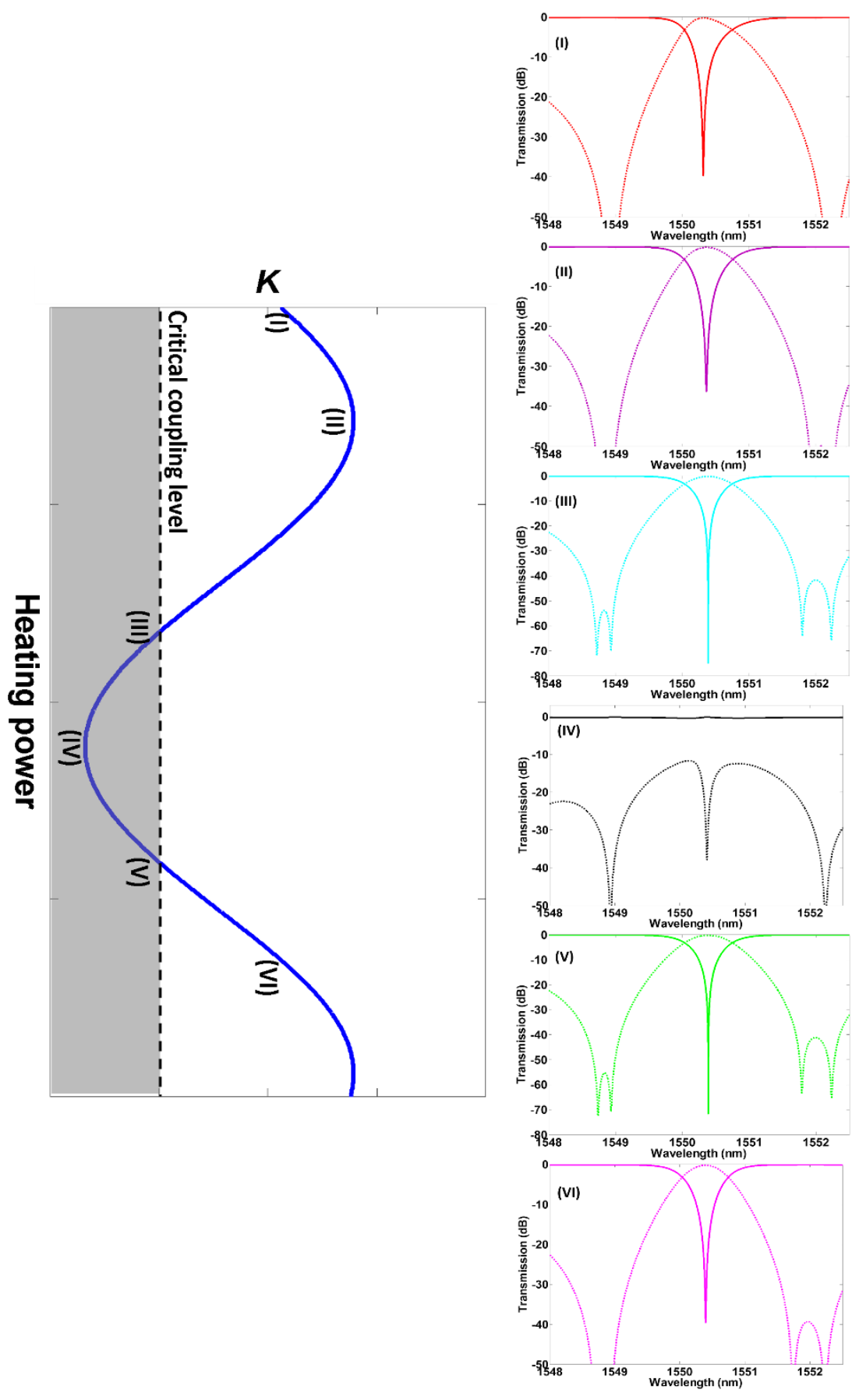

Figure 2.21: (Left) Tuning of $\kappa$ with heater power: the dashed line marks the critical coupling level; (I), (II), (III), (IV) and (V) mark the states of initial, maximal, critical, minimal and critical coupling, respectively. (Right) Corresponding through (solid lines) and drop (dotted lines) ports spectra. 
As commented before, by tuning both coupling coefficients, the bandwidth can be varied while maintaining a high extinction ratio. Moreover, in order to tune the resonance into the narrowest bandwidth and maximum extinction ratio, the heating power applied to the heaters must satisfy the conditions that the output coupling is minimum $\left(\boldsymbol{\kappa}^{\prime}=0\right)$ and the extinction ratio is maximum $(\kappa \sim \alpha)$. As shown in Figure 2.22 , by applying different heating powers to each heater (heaters 1 and 2 in Figure 2.23), bandwidth is tuned while the extinction ratio remains at a high value.
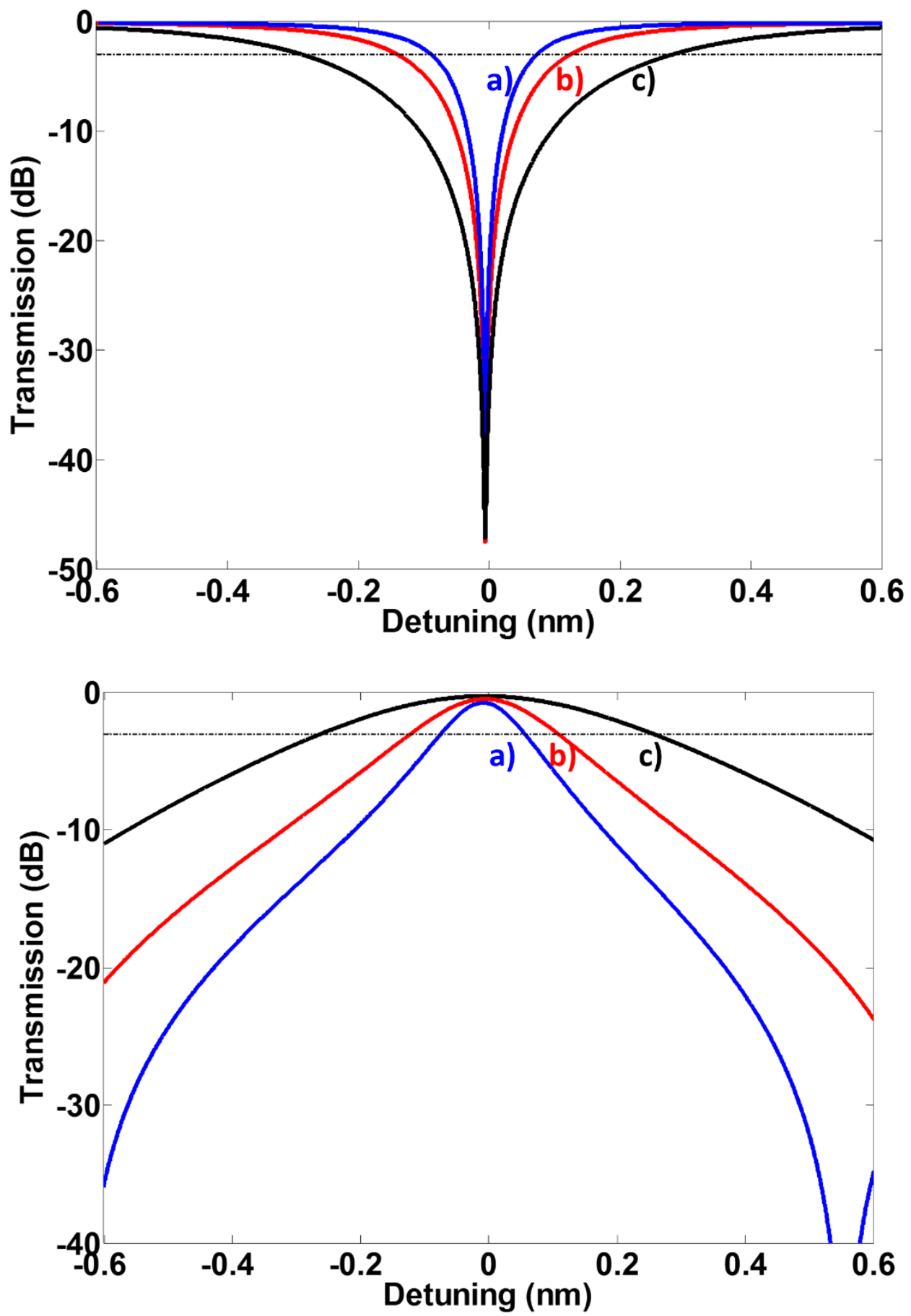

Figure 2.22: Tuning both coupling coefficients in order to achieve a tunable bandwidth while maintain a high extinction ratio. (Up) Through (Down) Drop ports spectra. The dotted line corresponds to $-3 \mathrm{~dB}$ transmission. 
As it can be seen from the figure above, there is a trade-off between the bandwidth and the insertion losses in the drop port, since as the bandwidth becomes more selective, the insertion losses increase.

\subsubsection{Characterization and Performance}

Regarding the fabrication process, which was carried out at Laboratory of Electronics, Technology and Instrumentation (LETI) facilities, on SOITEC optical SOI with $220 \mathrm{~nm}$ Si on $2 \mu \mathrm{m} \mathrm{BOX}$, the process starts with the deposition of 100nm High Temperature Oxide (HTO) on top of the silicon layer. The gratings and the waveguide arms are first patterned, followed by RIE silica etching with $\mathrm{C} 4 \mathrm{~F} 8$, which defines a hardmask. The silicon is then partially etched $(65 \mathrm{~nm})$ with $\mathrm{HBr}$ and controlled by ellipsometry in order to define precisely the grating teeth depth. In the second lithography step, the gratings are protected by the resist and the remaining hardmask serves for the waveguides in a selfalignment process. Then a full silicon etch down to the box completes the waveguide fabrication. A $400 \mathrm{~nm}$ thick $\mathrm{SiO} 2$ was deposited and a deposition and etching of 100nm of Ti/TiN defined the heaters. Then after deposition of $500 \mathrm{~nm}$ of $\mathrm{SiO}_{2}$ and two-step openings, the electrodes were defined by Ti/TiN/AlCu metal stack deposition and $\mathrm{Cl} 2$ etching. Figures 2.23(a) and (b) depict a schematic and a SEM image of the fabricated device, respectively.
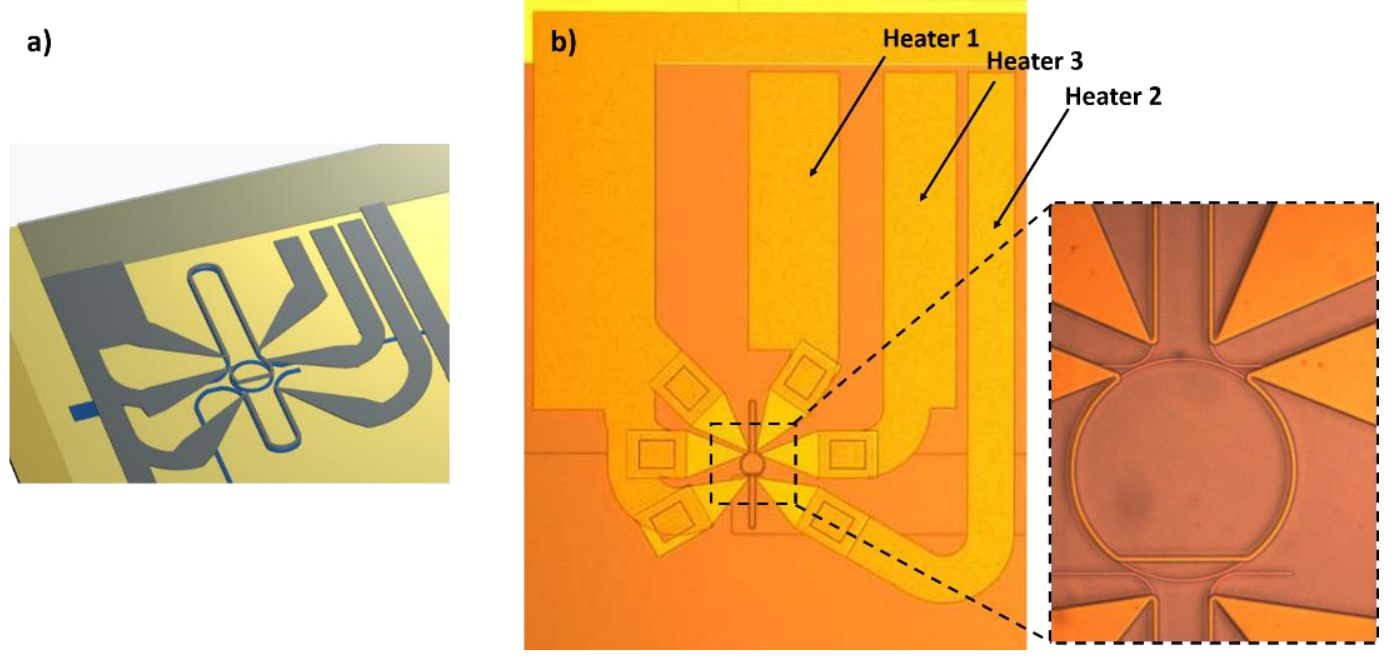

Figure 2.23: (a) Schematic and (b) SEM image of the fabricated device. Heaters 1, 2 and 3 are used for tuning the coupling coefficient for through port, drop port, and wavelength, respectively.

For the chip characterization at Nanophotonics Technology Center's (NTC) laboratories, a continuous-wave light was generated by a tunable external cavity laser (ECL) with a resolution of picometers. Measurements were performed with a single mode fiber tilted under an angle of 13 degrees relative to the surface normal of the grating. The input polarization state was firstly set by adjusting an external polarization controller and monitoring the output power measured in a reference straight waveguide. The light was coupled to the chip through the grating coupler using manual nano-positioners. For the tunable device characterization, individual DC probes were connected to the chip using manual micro-positioners, as shown in Figure 2.24. 


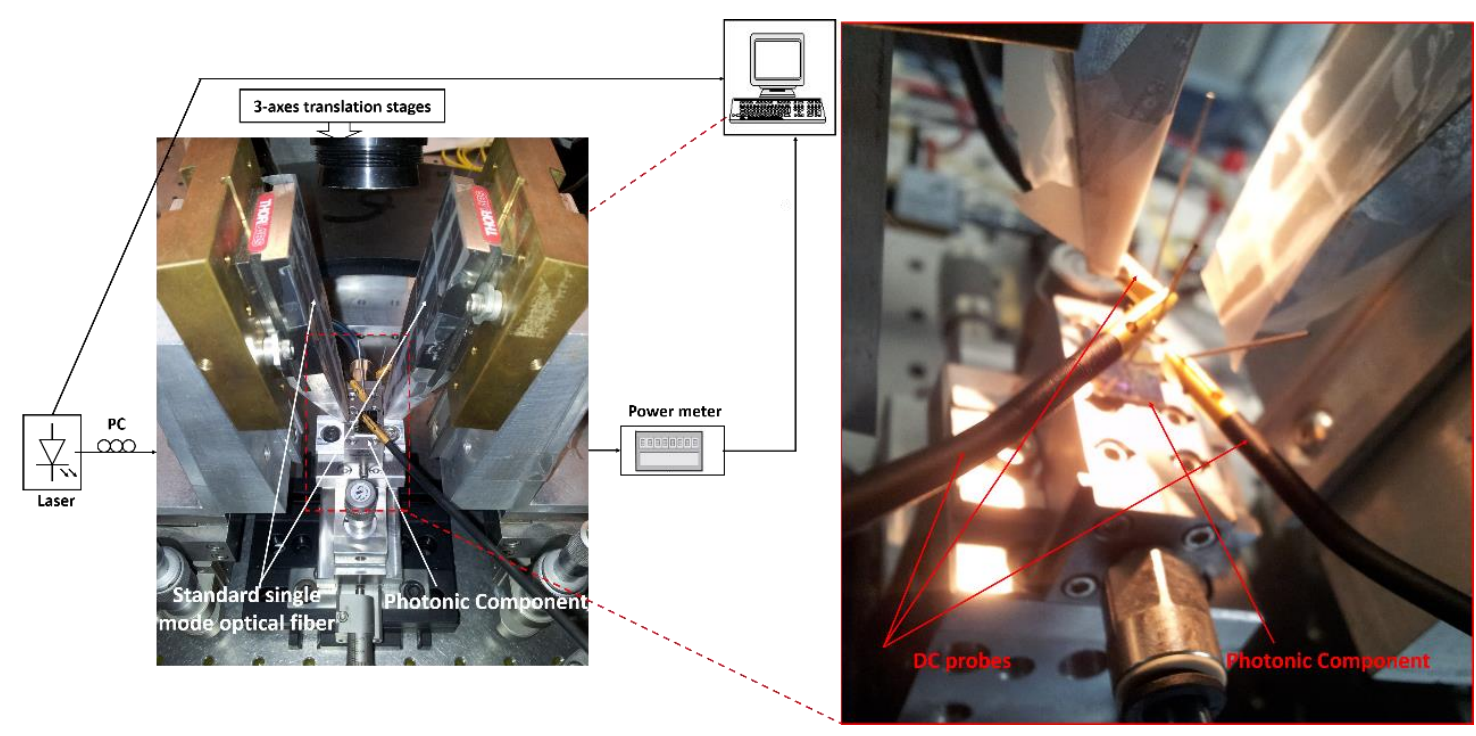

Figure 2.24: Image of the setup used to characterize the tunable DEMUX, using DC probes

As commented in the previous section, two optical carriers separated $60 \mathrm{GHz}$ at the demultiplexer input have to be filtered by centring one of them at a ring resonance, so each one of them can be processed independently from the other one, with a minimum extinction ratio of $25 \mathrm{~dB}$ and $30 \mathrm{~dB}$ for the through and drop ports respectively.

For a complete control of the filter, three microheaters are included for controlling the central wavelength (heater 3 in Figure 2.23(b)), the extinction ratio (heater 1 in Figure 2.23(b)), and the $Q$-value/bandwidth (heater 2 in Figure 2.23(b)).

Figure 2.25 shows different transmission curves for the through port, corresponding to different applied voltages over the microheater located above the ring resonator (heater 3 in Figure 2.23(b)). The figure clearly demonstrates the ability to tune the central wavelength of the demultiplexer. A $40 \mathrm{GHz}$ detuning is achieved by applying a total of $5 \mathrm{~V}$. The filtered showed here corresponds to the double tuned RR configuration as shown in Figure 2.20(a), however the same result is achieved for the single tuned RR.

Theoretically, the extinction ratio of the resonance should not be affected, only the central wavelength, but this slight change of the ER is due to the closeness of the heater to the coupling regions (see Figure 2.19(b) and Figure 2.20(b)). When the heater is active the heat does not only dissipate down towards the ring resonator, but also sideways toward the coupling regions. This can be avoided by either increase the closest distance to these sensitive regions and by making trenches on both sides of the waveguide, creating an air isolation ditch.

Once the control of the central wavelength of the demultiplexer is demonstrated, the next step is to tune the extinction ratio by using the thermo-optic effect in the heater corresponding to the through port coupling (heater 1 in Figure 2.23(b)). 


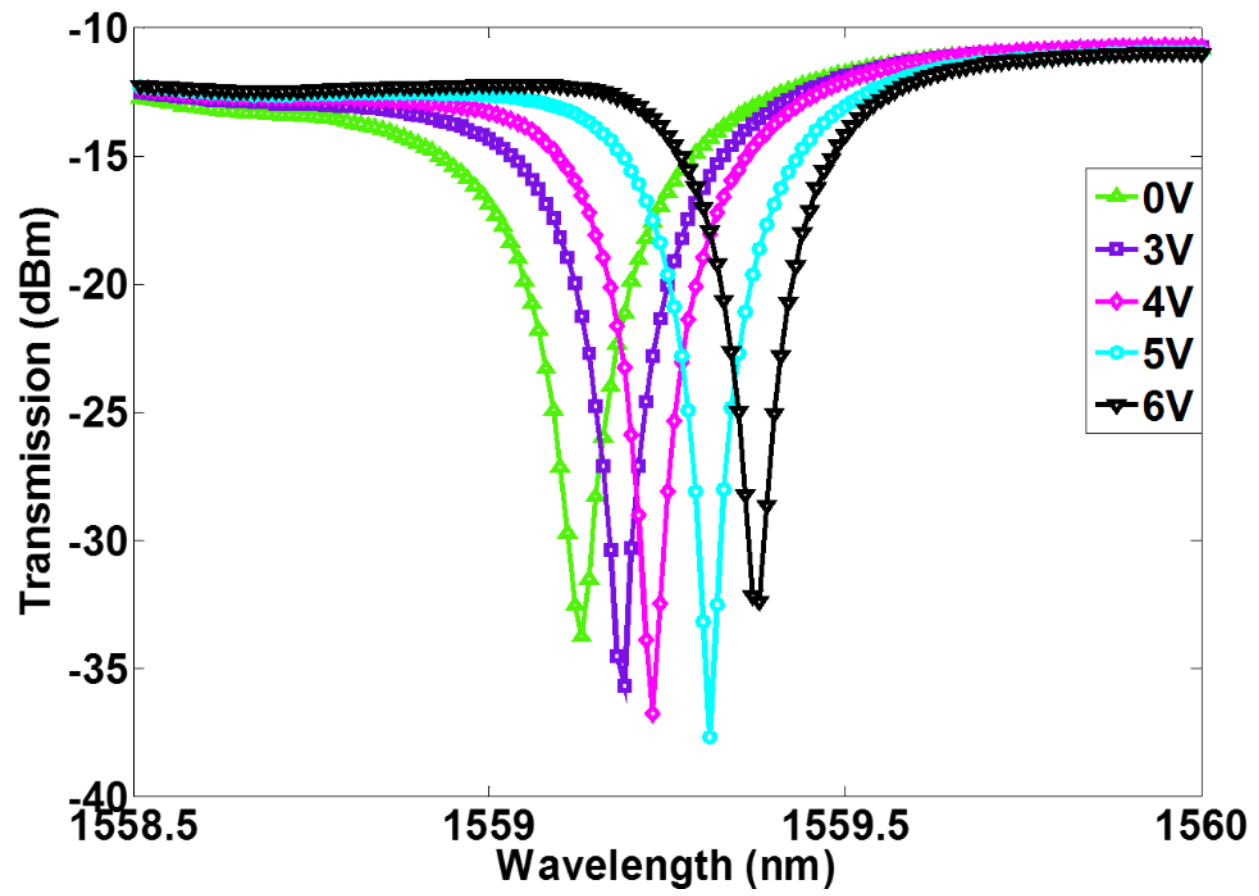

Figure 2.25: The control of the wavelength shift of the demultiplexer filter. The 5 graphs correspond to different applied voltage to the heater; from left to right, $0 \mathrm{~V}$ ( $\Delta$-green), $3 \mathrm{~V}$ ( $\square$-purple), $4 \mathrm{~V}(\diamond$-pink), $5 \mathrm{~V}$ (o-cyan) and $6 \mathrm{~V}$ ( $\nabla$-black) respectively.

To achieve a maximized ER the coupling coefficient should be equal to the losses in the ring $(\kappa \sim \alpha)$. Figure 2.26 and Figure 2.27 show the transmission spectra measured at the through and drop output ports, respectively.

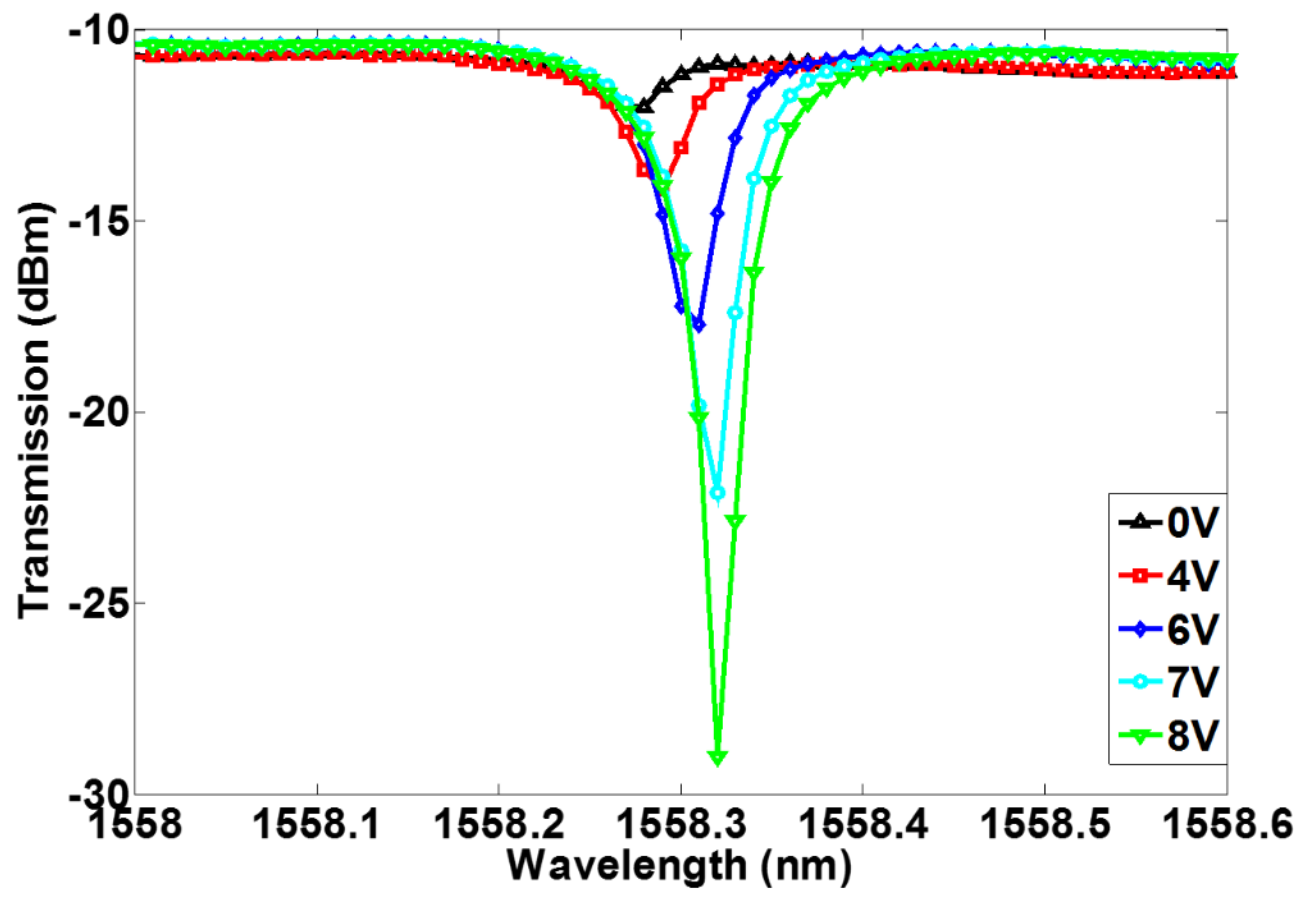

Figure 2.26: The transmission spectra for the through port of the demultiplexer when modifying the through coupling coefficient by applying different voltages to the heater. 
It is clearly shown in Figure 2.26 how the extinction ratio can be increased from a few $\mathrm{dBs}$ to close to $20 \mathrm{dBs}$ by applying $8 \mathrm{~V}$ to the micro-heater, agreeing with the expected behaviour from simulations. For the drop port shown in Figure 2.27 the extinction ratio is in this case also increased to reach more than $30 \mathrm{dBs}$. However, insertion losses are around $10 \mathrm{~dB}$ higher than in the through port. The extra insertion loss for the drop port is due to mainly the coupling losses. Moreover, the measured bandwidth is also tuned due to the closeness of the heaters, and as commented previously, by creating an air isolation ditch, this can be avoided.

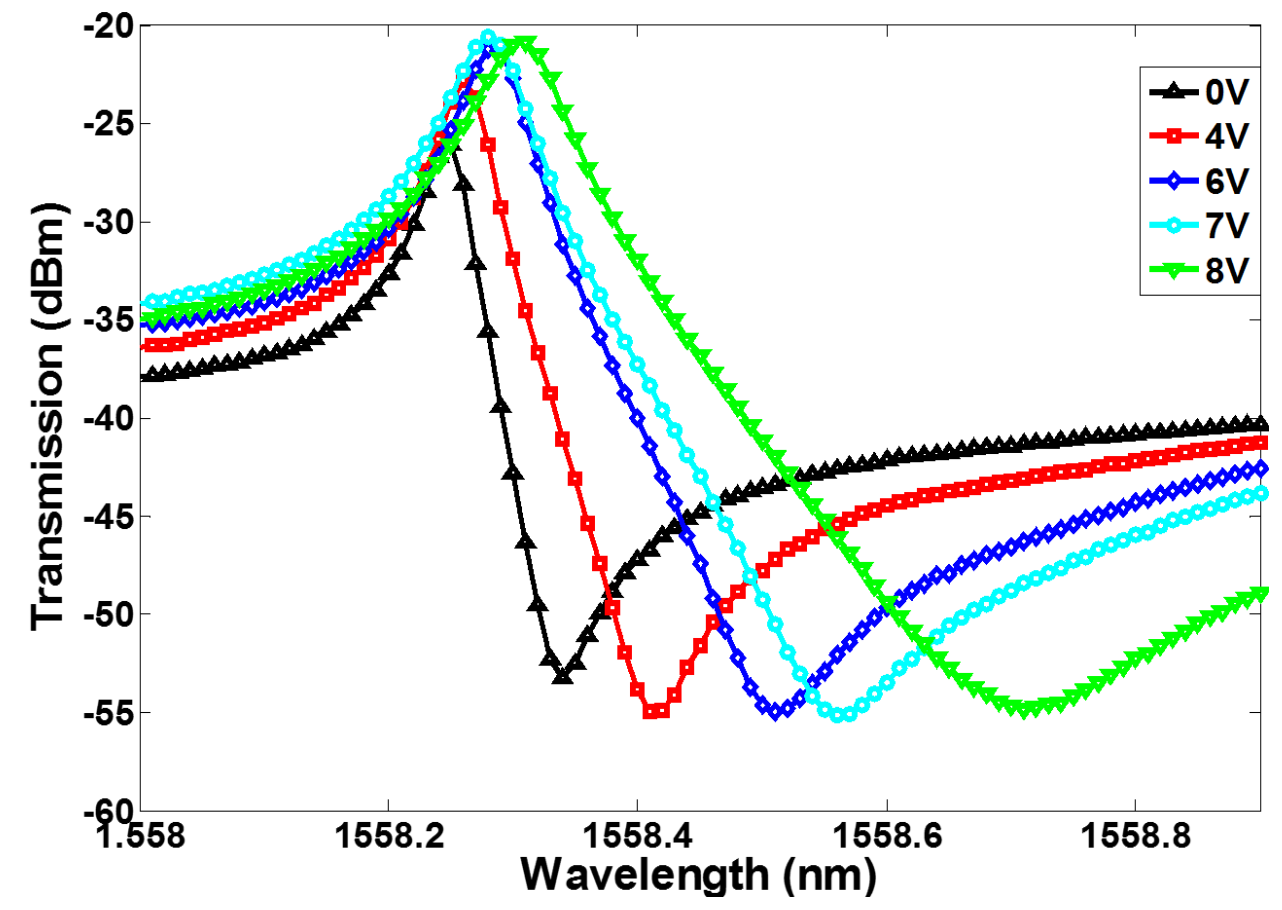

Figure 2.27: The transmission spectra for the drop port of the demultiplexer when modifying the coupling coefficient to the through waveguide by applying different voltages to the heater.

Once the tunability of extinction ratio is demonstrated by modifying the through coupling coefficient while maintaining constant the drop coupling coefficient, by using the doubled-tuned ring resonator, shown in Figure 2.20, the extinction ratio and the quality factor (bandwidth) of both the through and drop ports will be tuned simultaneously, in order to achieve the target specifications indicated in Table 2-1.

Figure 2.28 shows how, for two wavelengths separated $60 \mathrm{GHz}$, without no tuning mechanism, specifications are not accomplished for the drop port $(E R=10.43 \mathrm{~dB})$, but for the through port, the ER presents an almost valid value $(20.5 \mathrm{~dB})$ for the target specification $(25 \mathrm{~dB})$. By applying $7 \mathrm{~V}$ and $4 \mathrm{~V}$ to the drop and through micro-heaters respectively, the through port still presenting a value close to the specifications although the increase in ER is not significant $(E R=21.3 \mathrm{~dB})$, but the drop port $E R$ increases in $17.48 \mathrm{~dB}$, reaching $27.91 \mathrm{~dB}$ and almost satisfying the specifications $(30 \mathrm{~dB})$. Insertion loss in the drop port is also improved with respect to the no tuning response, and taking into account that the input/output grating couplers show a coupling loss of about $6 \mathrm{~dB}$, 
the actual insertion loss in the drop port is close to the target specification, reaching a value of about $3.75 \mathrm{~dB}$.
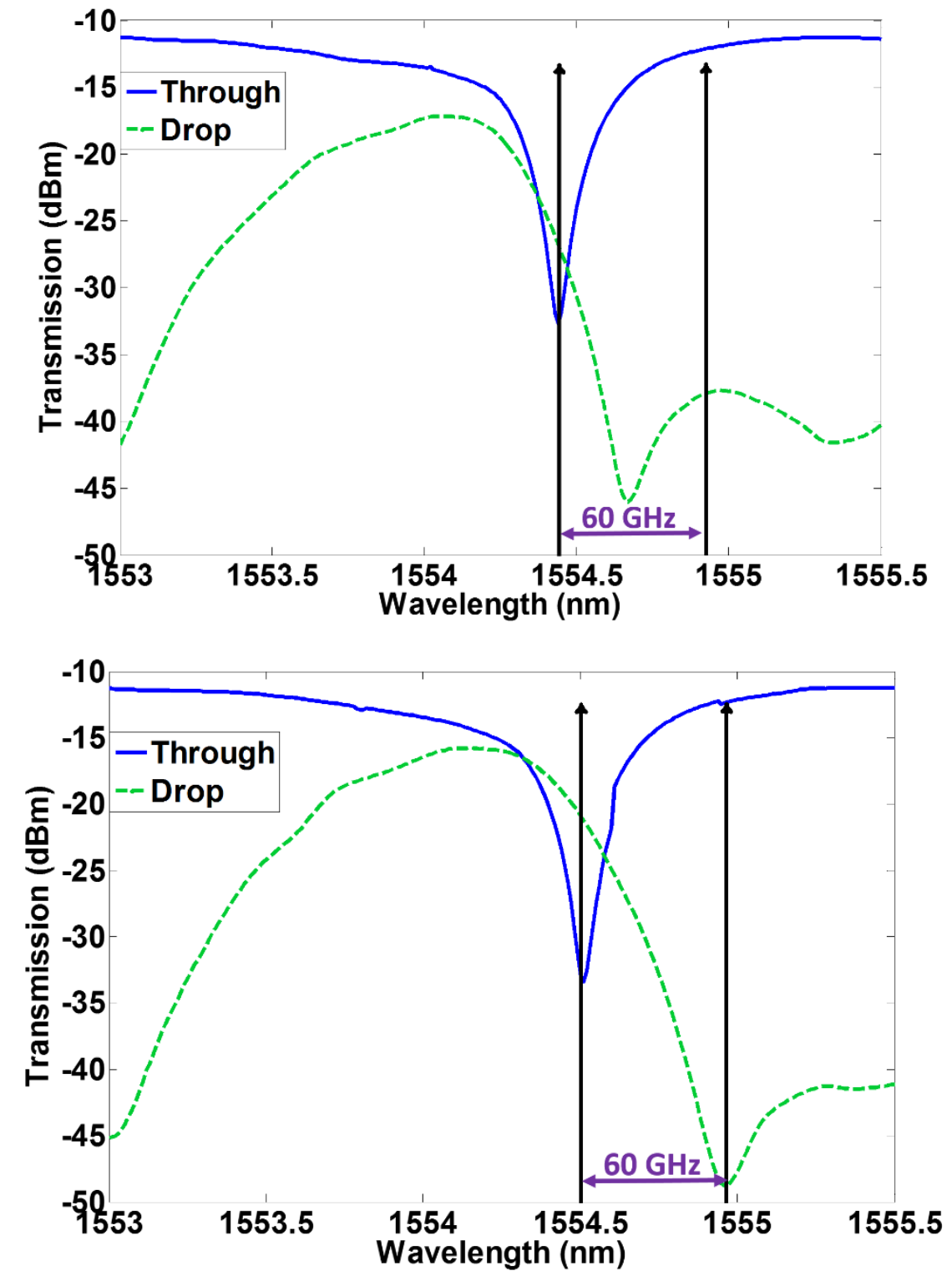

Figure 2.28: (Up) Drop (dashed line) and through curves without any tuning mechanism. (Down) Tuned drop (dashed line) and through curves, where the specification values are almost achieved.

\subsection{DQPSK Modulator}

\subsubsection{State Of The Art}

Silicon modulators based on microring structures have been proposed to achieve phase modulation [ZHA08, INT11]. Experimental demonstrations of $5 \mathrm{Gbit} / \mathrm{s}$ error-free differential PSK (DPSK) modulation [PAD11] and $20 \mathrm{Gbit} / \mathrm{s}$ QPSK modulation, but without successful error-free demonstration, have been recently reported [DON12c]. 
Ring based modulators have unique features in terms of small footprint and low drive voltage. However, the optical bandwidth or range of useful wavelengths for modulation is much lower than compared to conventional Mach-Zehnder modulators (MZM) that in turn implies that the modulator performance is more sensitive to fabrication tolerances. Thus, some tuning mechanism, which is usually based on the thermo-optic-effect [DON12c] becomes mandatory increasing power consumption and complexity of the transmitter.

Very recently, the first works dealing with phase modulation in silicon MZM have also been reported. A silicon based dual-drive nested MZM for QPSK modulation was firstly demonstrated at $20 \mathrm{Gbit} / \mathrm{s}$ [OGA12]. However, a poor system constellation was achieved due to the low extinction ratio and unbalanced output optical power at the MZMs. A higher modulation speed, $50 \mathrm{Gbit} / \mathrm{s}$ QPSK, has also been demonstrated by using a singledrive nested MZM but no error-free modulation was achieved [DON12a]. However, in the last months, many research groups have been working in the design and fabrication of silicon MZMs for PSK as described in [OGA12] and an experimental realization of 50 Gbit/s QPSK and 112 Gbit/s DP-QPSK modulation were reported in [DON12a] and [DON12b], respectively.

Some of the latest works in this field were presented in the last Optical Fiber Communication Conference and Exposition and National Fiber Optic Engineers Conference (OFC/NFOEC) held in San Francisco, California, March 2013. 20 Gb/s NRZ DPSK using a silicon- based MZM, with drive signals of $8 \mathrm{~V}$ peak-to-peak amplitude, was demonstrated [GOI13a]. From the same research group, a $44.6 \mathrm{Gbit} / \mathrm{s}$ DQPSK and 50-to$64 \mathrm{Gbit} / \mathrm{s}$ QPSK modulation demonstration using low loss nested Silicon MZM with fiber-to-fiber loss of 10dB were presented [GOI13b]. Error-free transmission is claimed, but using forward error correction (FEC). Moreover, an error floor can be seen in the presented results, as shown in Figure 2.29(a). Figure 2.29(b) shows the measured BER as function of the optical signal to noise ratio presented in [DON13], where $56 \mathrm{Gbit} / \mathrm{s}$ QPSK modulation was generated by using silicon microring based modulators with thermal control elements.

a)

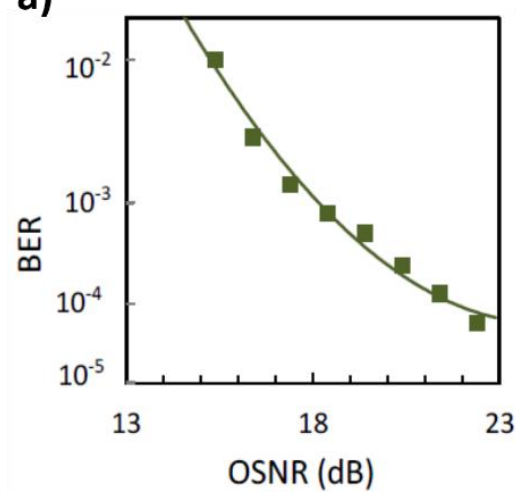

b)

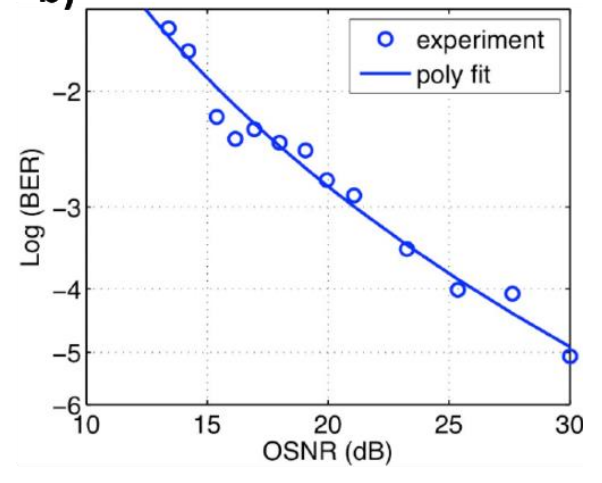

Figure 2.29: BER as function of the optical signal to noise ratio (a) 44.6Gb/s MZM-DQPSK presented in [GOI13b]. (b) 56-Gb/s microring-QPSK [DON13] 
Another work presented in the last OFC/NFOEC, but more focused for long-haul optical communication networks is [MIL13], where a transmission up to $2427 \mathrm{~km}$ SSMF using a silicon photonic modulator in a coherent optical polarization multiplexed $112 \mathrm{Gbit} / \mathrm{s}$ QPSK (DP-QPSK) system. Their implementation is based on a metal-oxidesemiconductor (MOS) capacitor. The amount of voltage difference across the Silicon Insulator Silicon CAPacitor (SISCAP) structure determines the charge accumulation and, through the free carrier effect, this determines the amount of phase modulation. The SISCAP structure is used for each arm of each MZM. The high charge density being centred in the active region of the optical mode enables highly efficient modulation, resulting in a $\mathrm{V}_{\pi} \mathrm{L}_{\pi}$ of less than $2 \mathrm{~V} \cdot \mathrm{mm}$.

\subsubsection{Design, fabrication and characterization}

A high-speed DPSK modulation using a silicon push-pull operated dual-drive MZM based on carrier depletion has been demonstrated [AAM13a], validating the potential to achieve higher order modulation formats, such as, DQPSK, by arranging the presented MZM in a nested configuration, as already described in Section 2.2.

The GDS design and fabricated MZM at LETI's facilities is shown in Figure 2.30(a) and (b) respectively. Multimode interference couplers (MMI) were used as input/output $3 \mathrm{~dB}$ couplers. The silicon waveguide core has a height of $220 \mathrm{~nm}$, a width of $450 \mathrm{~nm}$, and a slab thickness of $100 \mathrm{~nm}$, as illustrated in Figure 2.30(c). Optical phase modulation is achieved by depleting the majority carriers from a reverse biased p-n junction [THO12] with doping concentrations of $1.6 \cdot 10^{17} \mathrm{~cm}^{-3}$ in the p-type region and $8 \cdot 10^{17} \mathrm{~cm}^{-3}$ in the $\mathrm{n}$ type region.

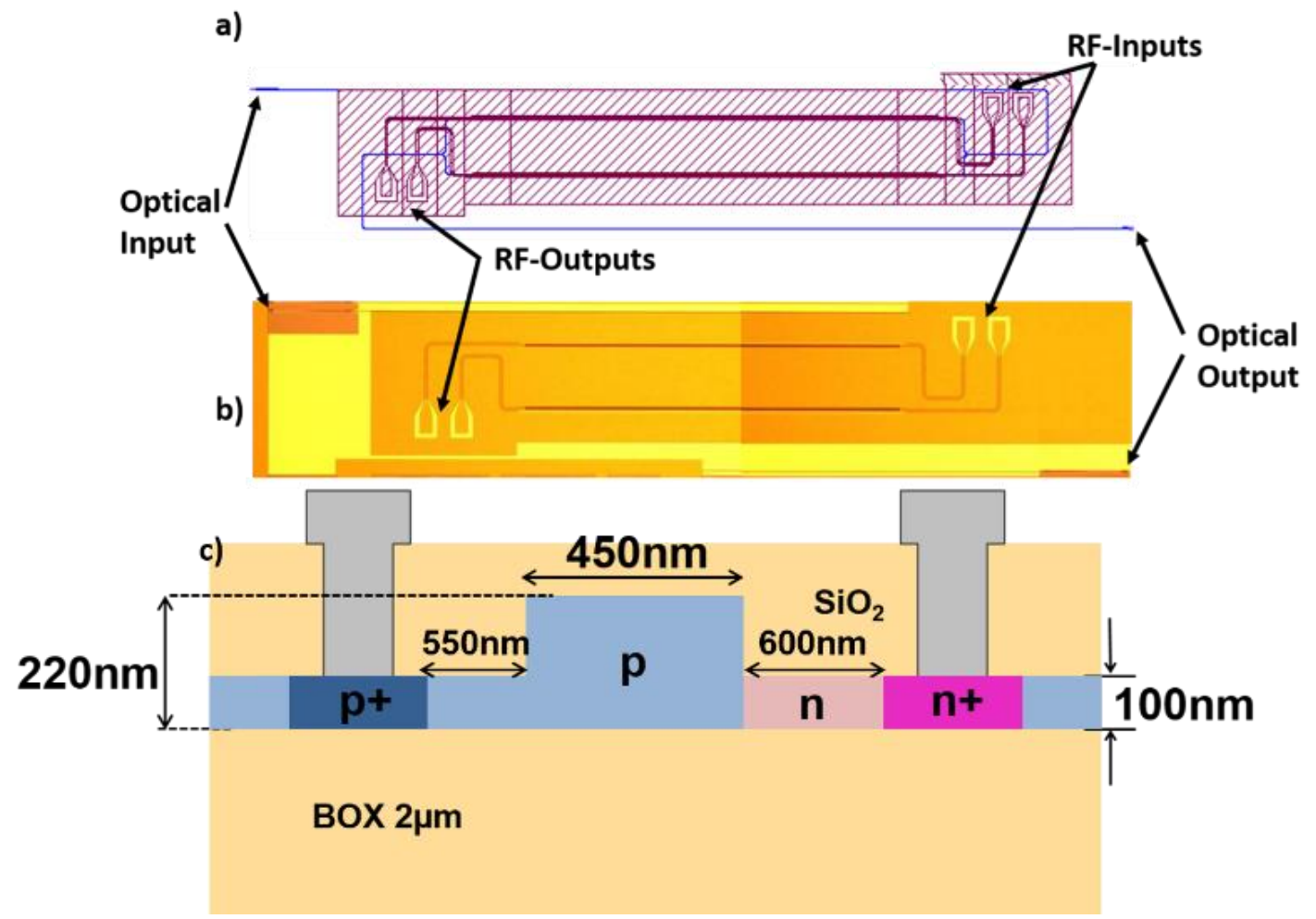

Figure 2.30: DPSK modulator. (a) GDS design, (b) optical photograph of fabricated device and (c) crosssection of the pn junction. 
The travelling-wave electrodes are formed by depositing a compound $\mathrm{AlCu}$ layer on top of highly doped $\mathrm{p}+$ and $\mathrm{n}+$ regions with concentrations of $1 \cdot 10^{20} \mathrm{~cm}^{-3}$. A dual-drive electrode configuration was chosen for push-pull operation, which allows producing the required $\pi$-phase shift for DPSK modulation when the phase shifters are biased at zero amplitude and driven by digital data signals with opposite polarity and a peak-to-peak voltage of $\mathrm{V}_{\pi}$. The two electrodes have the same length to avoid a delay between the noninverted and inverted digital data signals.

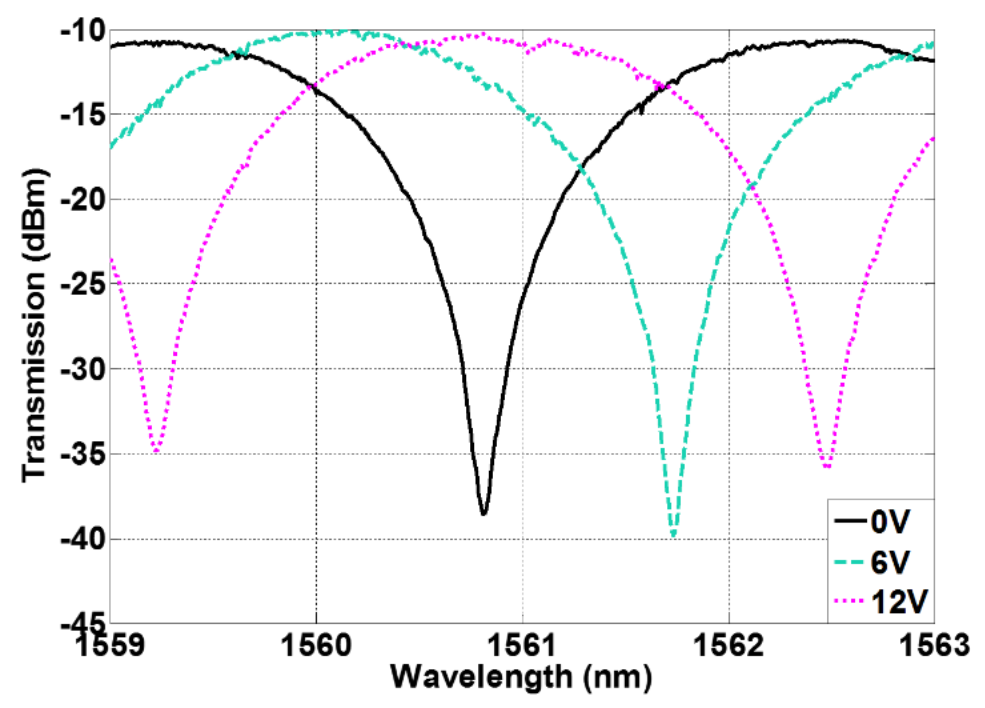

Figure 2.31: Normalized transmission spectra of the MZM with different bias voltages.

First, at the NTC's facilities, the transmission spectra of the MZM were obtained for different applied voltages. Figure 2.31 shows the spectra normalized to a reference waveguide. The insertion loss of our DPSK modulator, including phase shifter and MMI losses, is about $10 \mathrm{~dB}$ and the free spectral range (FSR) is $3 \mathrm{~nm}$. Different voltages between $0 \mathrm{~V}$ and $12 \mathrm{~V}$ were applied to the MZM. The extinction ratios under these DC conditions were close to $30 \mathrm{~dB}$. At $12 \mathrm{~V}$ the curve was shifted exactly one half FSR, as it can be seen in Figure 2.33, marking our $\mathrm{V}_{\pi}$ value and giving rise to a $\mathrm{V}_{\pi} \cdot \mathrm{L}$ product about $3.6 \mathrm{~V} \cdot \mathrm{cm}$. Compared to previous works on silicon MZM for PSK modulation, the $\mathrm{V}_{\pi}$ is only slightly higher than the $10 \mathrm{~V}$ reported in [DON12a] but in contrast the modulation length is reduced down to $3 \mathrm{~mm}$, half of the length reported in [OGA12, DON12a], thus significantly reducing the device footprint. Insertion losses are around $5 \mathrm{~dB}$ higher but they could be reduced via a better optimization of the separation between the high doping regions and the optical waveguide while minimizing the impact on the modulator performance.

The next step is to characterize the DPSK modulation using the measurement setup shown in Figure 2.32. The input light emitted by an external cavity laser (ECL) is coupled from a standard single mode fiber to the chip via grating couplers. The polarization was optimized and set to a TE polarization using a polarization controller (PC). Before being launched onto the chip, the optical signal was amplified by an erbium-doped fiber amplifier (EDFA), and filtered by a 3nm wide tunable optical filter. Digital data signals were generated from a pseudorandom binary sequence pattern generator with a pattern 
length of $2^{7}-1$, delivered by a bit pattern generator (BPG) connected to an external clock. The signals were appropriately decorrelated and aligned before being fed to the electrodes with $8 \mathrm{~V}$ peak-to-peak voltage. A double RF signal probe with GSGSG configuration was used to drive the MZM, while another double RF signal probe with $50 \Omega$ terminators was applied at the electrode output (see Figure 2.30(a) and (b)). A reverse DC bias was applied to the phase shifters for operation in carrier depletion. Separate DC sources were used to adjust the bias level of the phase shifters independently using bias-tees. The optical modulated DPSK signal was once again amplified after coupling out of the chip, and filtered before being visualized in a digital communication analyzer (DCA) for capturing the modulated eye.

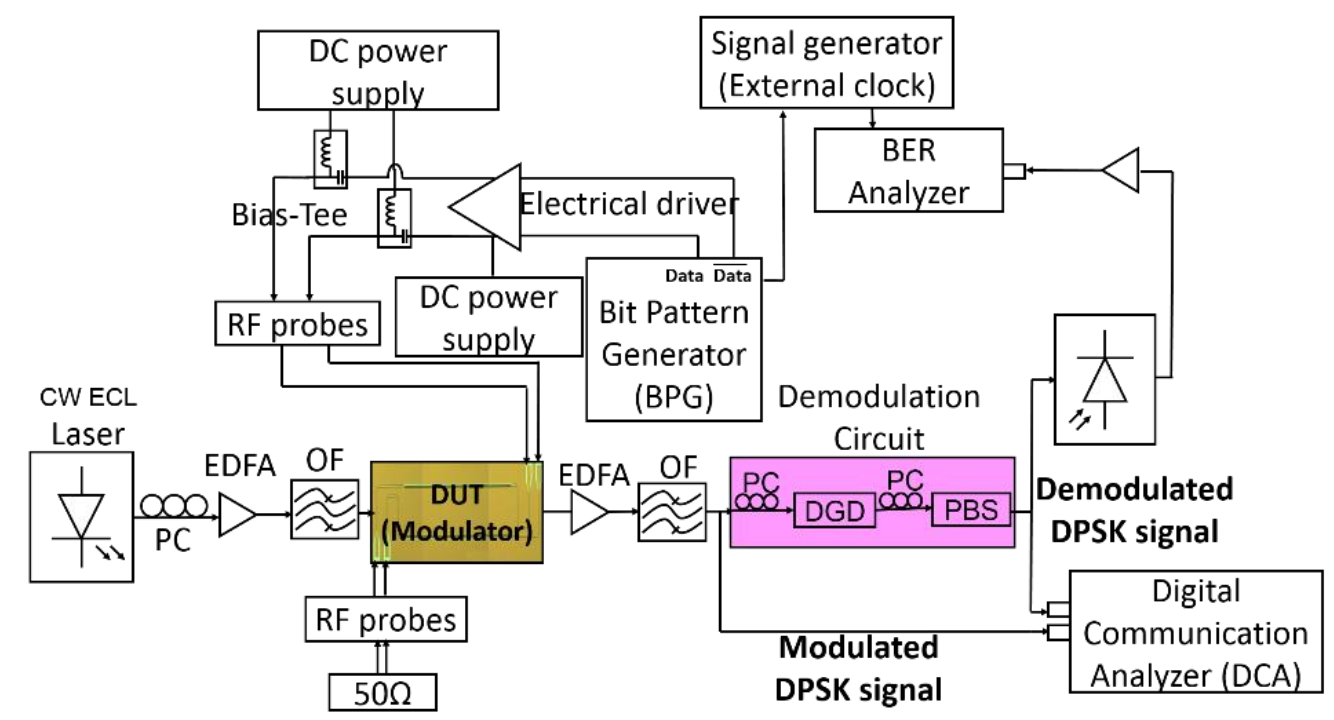

Figure 2.32: Schematic of the experimental set-up for evaluating the performance of the DPSK modulator.

In order to measure the bit error rate (BER), the optical DPSK modulated signal was passed through an external demodulation circuit, as depicted in Figure 2.32. The demodulation is based on a polarization delay interferometer [CHO05]. Using a PC, the polarization of the modulated signal is transformed into a linear polarized wave with the same intensity in the TE and the TM axes. The linear signal is then launched into a differential group delay (DGD). The DGD is a birrefrigent crystal that introduces a fixed differential group delay between the TE and TM polarization axes. In our demodulation circuit, the DGD has been used such it introduces a fixed delay between the TE and TM components that is equal or higher than 1 bit period of the modulated signal. Finally, the output signal is again adjusted in polarization with another PC and combined with a polarization beam splitter (PBS). In this way, the linear polarization is rotated $45^{\circ}$, so the same fraction of the TE and the delayed TM intensities match one of the polarization axis of the PBS. Hence, a combination of the signal with a 1 bit delayed signal is achieved. The demodulated signal is then simultaneously fed to the DCA and BER analyzer, as depicted in Figure 2.32. 


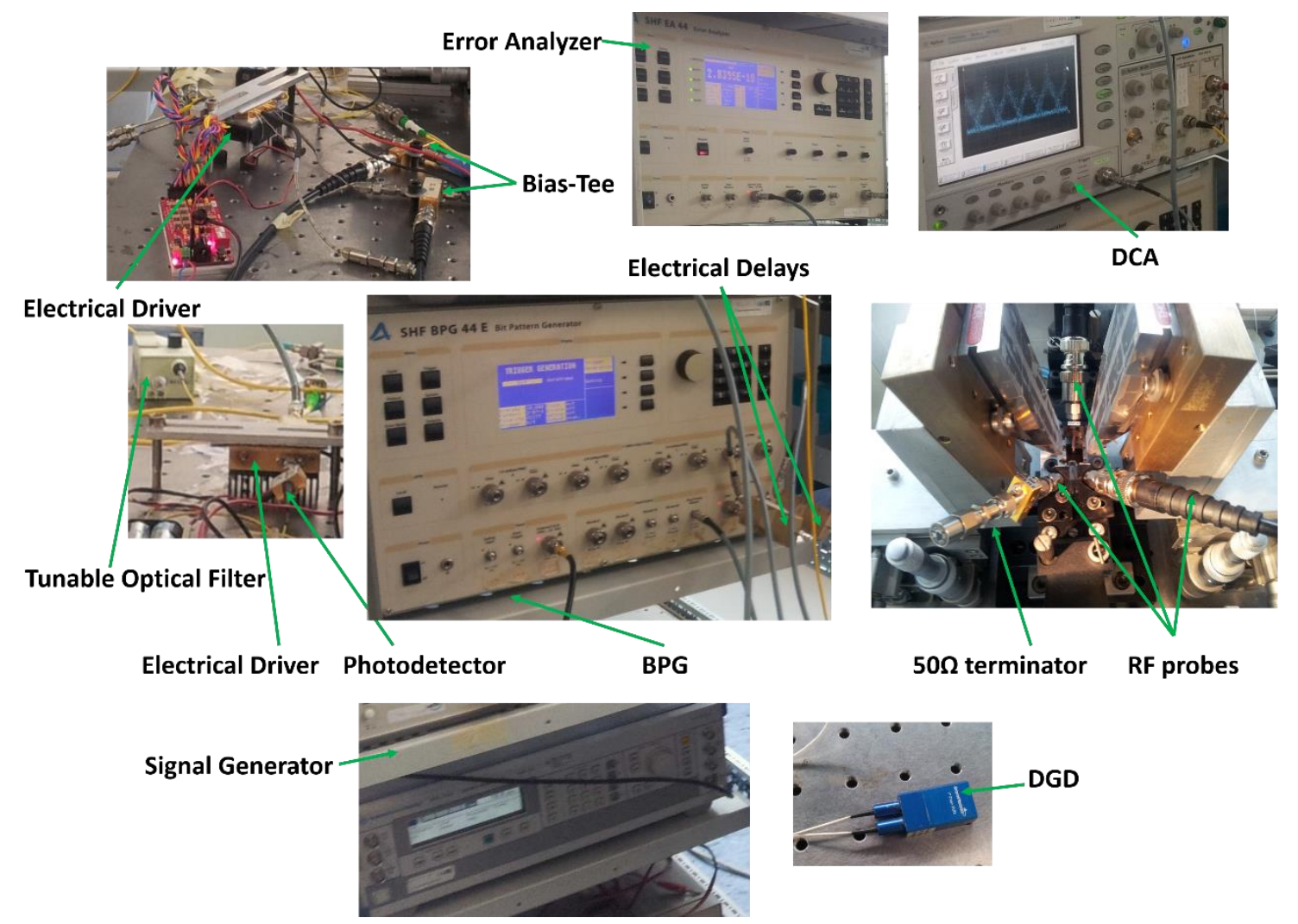

Figure 2.33: Images of some of the equipment used for the experimental characterization of the DPSK modulator.
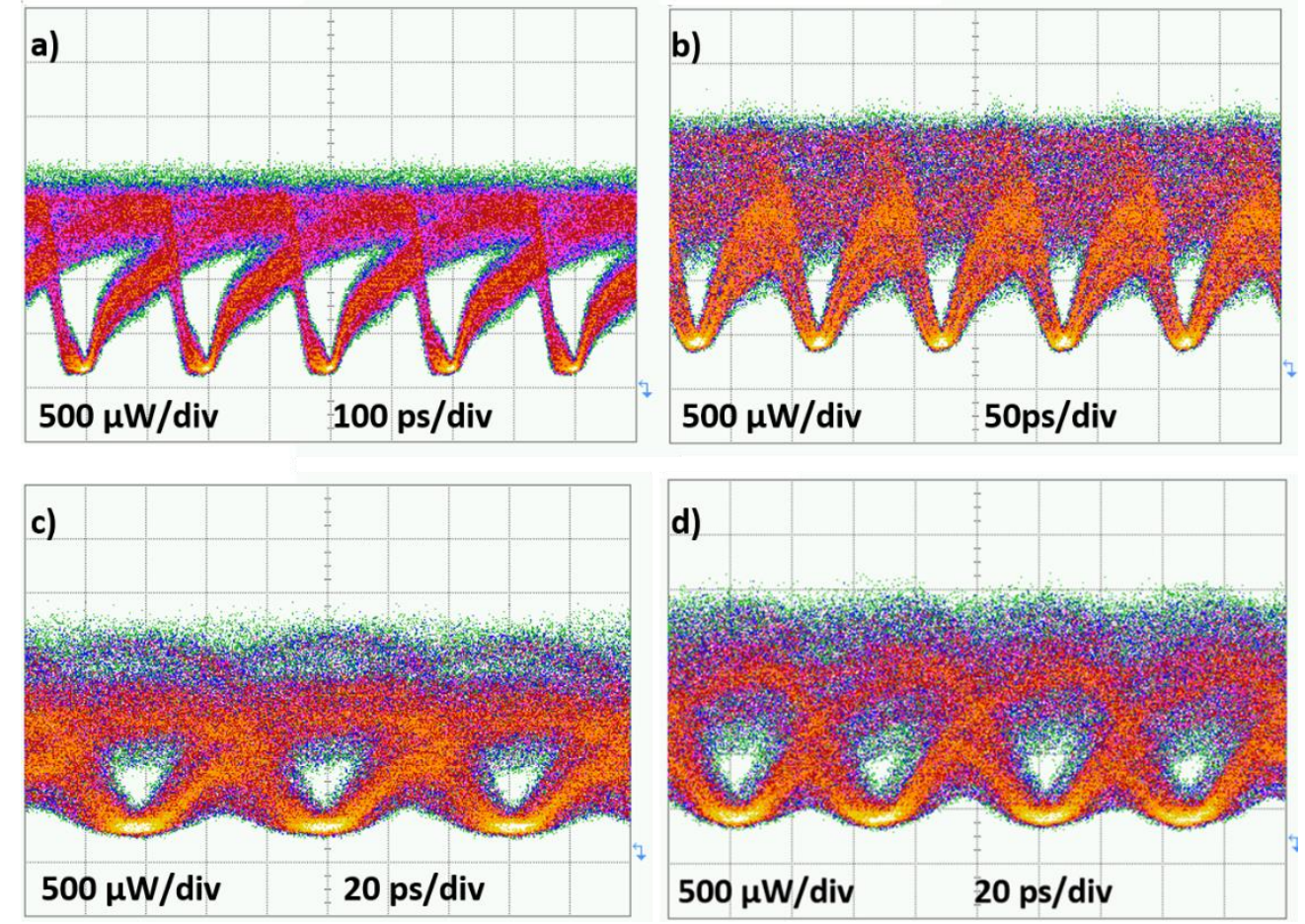

Figure 2.34: modulated DPSK eye diagram for (a) $5 \mathrm{Gbit} / \mathrm{s} \mathrm{(b)} 10 \mathrm{Gbit} / \mathrm{s} \mathrm{(c)} 15 \mathrm{Gbit} / \mathrm{s}$ and (d) $20 \mathrm{Gbit} / \mathrm{s}$.

The measured eye diagrams of the modulated DPSK signal for four different bit rates are shown in Figure 2.34. The noise is mainly due to the limitation in the drive voltage which 
is not high enough to achieve $\mathrm{V}_{\pi}$ in each phase shifter of the MZM (the driver only offers $66.6 \%$ of the required $\mathrm{V}_{\pi}$ ). However, clear demodulated eye diagrams, as depicted in Figure 2.35, were obtained for $5 \mathrm{Gbit} / \mathrm{s}$ and $10 \mathrm{Gbit} / \mathrm{s}$ by using the fixed DGD ( $\tau \sim 90 \mathrm{ps}$ ) and for $15 \mathrm{Gbit} / \mathrm{s}$ using a variable DGD, despite being unstable for BER measurements. Figure 2.35(a) and (b) shows the alternate-mark inversion (AMI) and Duobinary (DB) demodulated eye diagrams for the $5 \mathrm{Gbit} / \mathrm{s}$ modulation, while Figure 2.35(c) and (d) shows the AMI demodulated eye diagram for the $10 \mathrm{Gbit} / \mathrm{s}$ and $15 \mathrm{Gbit} / \mathrm{s}$ modulation bit rates, respectively. As it shown in these figures, very open eye diagrams were measured confirming the correct DPSK modulation for the measured bit rates.
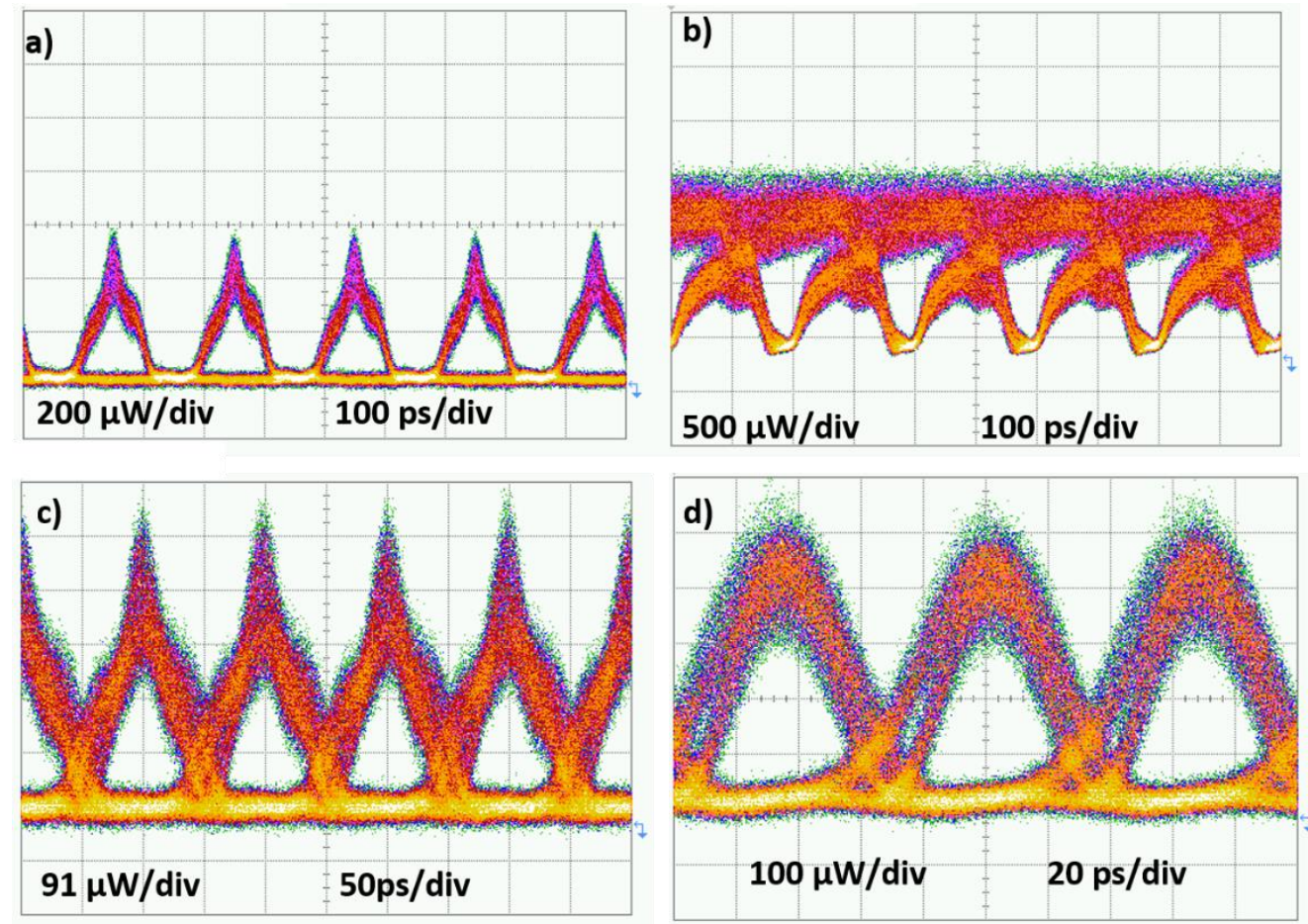

Figure 2.35: DPSK demodulated (a) AMI and (b) DB eye diagrams for 5 Gbit/s. AMI demodulated eye diagrams for (c) $10 \mathrm{Gbit} / \mathrm{s}$ and (d) $15 \mathrm{Gbit} / \mathrm{s}$.

The performance of the silicon DPSK modulator was further evaluated by measuring the BER but only at the two lower bit rates because of the limitation in the delay introduced by the fixed DGD since, unfortunately, the delay introduced by the DGD was too long for correctly demodulating the DPSK signal above $10 \mathrm{Gbit} / \mathrm{s}$ and therefore it was not possible to measure the BER for higher speeds.

As shown in Figure 2.36, error-free DPSK modulation, for 5 Gbit/s (o-blue curve) and $10 \mathrm{Gbit} / \mathrm{s}$ ( $\diamond$-red curve), is obtained. Furthermore, no error floor is observed in the results showing that inter-symbol interference (ISI) is not produced in the modulation due to carrier latency effects. Moreover, it must be commented that our modulator would most likely be good enough to achieve an error-free modulation at $20 \mathrm{Gbit} / \mathrm{s}$ and maybe even higher. When comparing the modulated eye diagram for $10 \mathrm{Gbit} / \mathrm{s}$ and $20 \mathrm{Gbit} / \mathrm{s}$ the noise level is about the same. Furthermore, it can be clearly seen that the transitions, in the 20 Gbit/s eye diagram, between the different states do not overlap which confirms that ISI would not occur when the right demodulation stage is used and thus the high speed 
operation of the DPSK modulator.

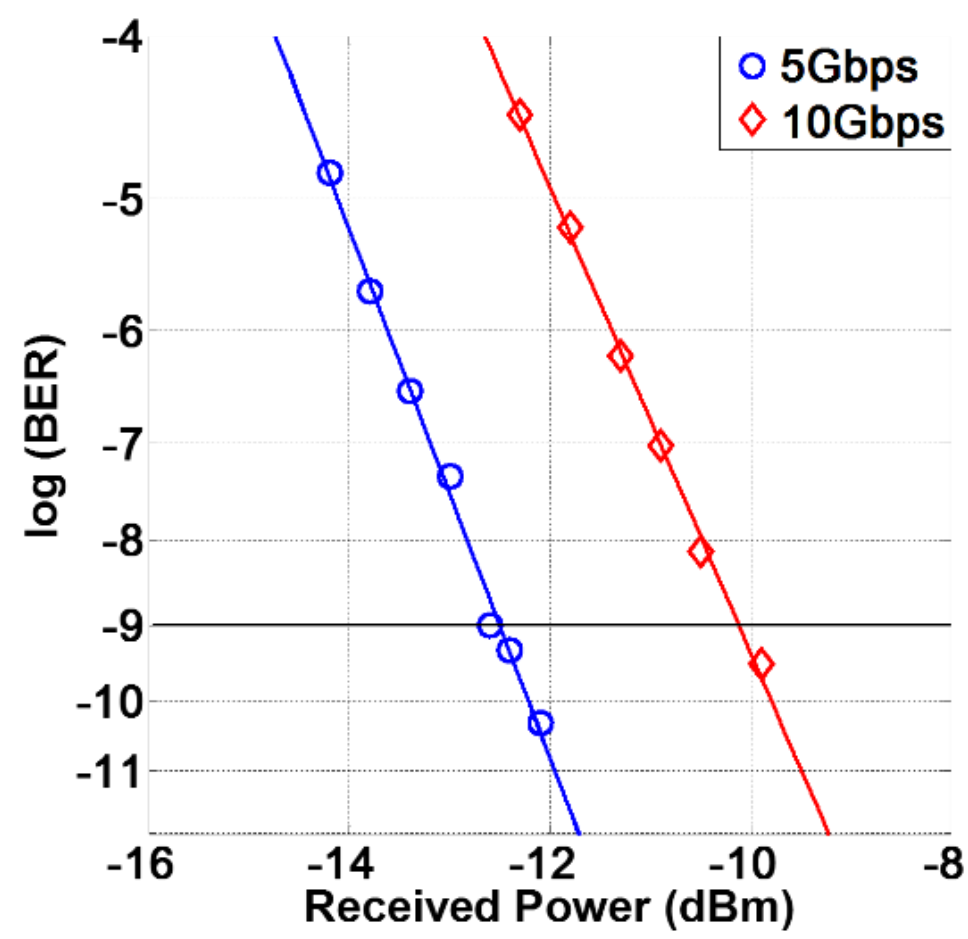

Figure 2.36: BER versus received power for 5Gbit/s ( $\circ$-blue curve) and 10Gbit/s ( $\diamond$-red curve) DPSK demodulation.

In summary, we have successfully demonstrated error-free DPSK modulation at $5 \mathrm{Gbit} / \mathrm{s}$ and $10 \mathrm{Gbit} / \mathrm{s}$ using a dual-drive silicon MZM. Furthermore, we have also shown the feasibility of the proposed MZM for $15 \mathrm{Gbit} / \mathrm{s}$ and $20 \mathrm{Gbit} / \mathrm{s}$ DPSK modulation. Finally, a fully integrated silicon transceiver could be implemented by combining the proposed MZM with an integrated silicon DPSK receiver [AAM12], which will be described in the next chapter. 
Transmitter 


\section{Chapter 3}

\section{Receiver}

\subsection{Introduction}

The DQPSK polarization insensitive receiver is formed by different building blocks that will described in the next lines.

First of all, it must be commented that coherent and incoherent detection methods enable high spectral efficiency modulation formats with electronic compensation of linear and nonlinear impairments. Regarding hardware complexity, coherent receivers require a local oscillator, which can make integration of the complete receiver difficult and expensive. This is especially the case when high-order modulation formats are used, which require low-linewidth lasers such as external cavity laser (ECL). ECLs are also not preferable when cost-efficiency is of primary concern, such as in access networks. In the other hand, differential modulation formats are less sensitive to laser phase noise [KIK10]; the differential beating in the receiver cancels out part of the phase noise, as it does not change so much over a symbol period. Incoherent receivers are also simpler in terms of digital signal processing (DSP), as already mentioned, as they do not require frequency and carrier and phase recovery.

As a technological platform, SOI presents a low-cost and compact solution for massproduction of highly integrated photonic devices and has shown good potential for implementing various formats of DPSK demodulation. The small bending radius of about 5 microns, alongside the monolithic integration of germanium photodetectors (Ge-PD) by using CMOS compatible process makes this technology very attractive for low-cost 
differential receivers, specifically in access networks at lower data speeds, such as 5 Gbit/s or $10 \mathrm{Gbit} / \mathrm{s}$. In order to deal with problems such as polarization dependent loss and polarization dependent frequency, the TE and TM polarization can be completely separated and processed individually using the diversity scheme shown in Figure 3.1. Different approaches have been proposed for differential silicon receivers, mainly based on the use of standard Mach-Zehnder delay interferometer (MZDI) [KRO11, FAR12, DOE12] or using microring resonators [XU12, DIN11]. While the ring resonator approach allows a very compact implementation, optimal performance usually requires a tuning mechanism [CHE07], increasing the complexity and power consumption of the receiver. Accordingly, the MZDI design seems to be the most suitable to be used in more complete systems [DOE12, SUZ12].

As commented previously, the proposed DQPSK receiver is polarization insensitive. The transparent polarization behaviour is given by a polarization diversity scheme [BAR07], in order to deal with some problems such as polarization dependent loss and polarization dependent frequency, so the transversal electric (TE) and the transversal magnetic (TM) polarizations are completely separated and processed individually. The first key building of the polarization diversity scheme is the polarization splitter to separate the random polarization coming from the input fiber. Once the two polarizations are separated, the TM component has to be rotated to achieve the TE one by the use of a polarization rotator. The proposed DQPSK receiver, in addition to the polarization diversity scheme, will also include a thermo-optically tunable MZI power splitter, in series with a MZDI, a compact $2 \times 4$ multimode interference coupler (MMI) acting as 90 degree hybrid and two balanced germanium photodetectors (Ge-PDs).

The principle of operation of a differential demodulator using a MZDI is to superpose two adjacent bits using a delay-line (DL) and the intensity of the superposed signal can be directly photodetected, converting the differential bit transition into an intensity variation of the signal. In order to maximize the sensitivity of the receiver, the output of the MZDI has to be balanced; but due to the losses in the DL, behaviour may result in unbalanced one. That is one of the reasons of introducing a tunable MZI switch in cascade with the demodulation circuit, so propagation losses can be compensated by actively tuning the power at the MZDI input, resulting in an increase in the extinction ratio of the MZDI. An alternative implementation based on two variable optical attenuators (VOAs) coupled to each waveguide of the MZDI has been proposed to balance the output power in a MZDI based receiver [SUZ12]. However, VOAs will introduce extra losses in addition to higher power consumption, if compared with our approach. To overcome the drawback of compactness when using a MZDI instead of microring resonator implementation, we will use compact spirals to implement the DL, so the footprint will be drastically reduced, and thereby the size of the receiver will be minimized to just a few times larger than a ring resonator based design.

The standard implementation of a DQPSK receiver with two parallel MZDI is to couple each MZDI to a 180 degree hybrid. According with the approach of minimizing our chip area, as well as the number of active controls needed for tuning, we will use a $2 \mathrm{x} 4 \mathrm{MMI}$ 
acting as a 90 degree hybrid, which inputs will be coupled to the MZDI outputs. Moreover, in this work, a zero bias balanced detection is used, so the complexity and the size of the receiver will be scaled down, once again, by excluding the need of a decoupling capacitor. A Ge-PD in pinpin configuration will be used, so additional wire bonding or metal connections are suppressed. Only few works have demonstrated zero bias Ge detectors selectively grown at the end of silicon waveguides [VIV12] or in surface illuminated configuration [JUT05].

As commented in previous chapters, this work is in the framework of the European project HELIOS [HEL]. The goal of this chapter is the development of a photonic DQPSK receiver at $10 \mathrm{Gbit} / \mathrm{s}$, and the performance requirements for each of its building block are described in the following table.

TABLE 3-1

BUILDING BLOCKS AND HELIOS PERFORMANCE REQUIREMENTS FOR DQPSK RECEIVER

\begin{tabular}{|c|c|c|}
\hline Device & Description & $\begin{array}{l}\text { Performance } \\
\text { Requirements }\end{array}$ \\
\hline Polarization Splitter & $\begin{array}{l}\text { Device responsible for the } \\
\text { separation of the input } \\
\text { polarization into its two } \\
\text { orthogonal components. }\end{array}$ & $\begin{array}{l}\text { ER }>\mathbf{2 0 d B} \text { for both output } \\
\text { ports. } \\
\text { IL }<\mathbf{3 d B}\end{array}$ \\
\hline Polarization Rotator & $\begin{array}{l}\text { Device which function is to } \\
\text { rotate the input polarization } \\
\text { state into the orthogonal } \\
\text { one. }\end{array}$ & $P C E>-1 d B$ \\
\hline 1-bit Delay Line (DL) & $\begin{array}{l}\text { Building block of the } \\
\text { MZDI. The delay depends } \\
\text { on the frequency of the } \\
\text { signal. For } 5 \mathrm{GHz} \text { signal, a } \\
200 \mathrm{ps} \text { DL is needed, } \\
\text { corresponding to } 18 \mathrm{~mm} \text { in } \\
\text { SOI single mode } \\
\text { waveguide technology }\end{array}$ & Loss $<5 \mathrm{~dB}$ \\
\hline $\begin{array}{c}\text { Balanced } \\
\text { Photodetectors (BPD) }\end{array}$ & $\begin{array}{l}\text { Balanced detection is used. } \\
\text { The output of the two BPD } \\
\text { is the two original RF } \\
\text { signals. }\end{array}$ & $\begin{array}{l}\text { Responsivity } R>0.8 \mathrm{~A} / \mathrm{W} \\
\text { Dark current } \\
I_{\text {dark }} \approx 10 \mathrm{nA}\end{array}$ \\
\hline
\end{tabular}




\subsection{Polarization Diversity Scheme}

\subsubsection{Introduction}

As already described in Chapter 1 Silicon on Insulator (SOI) is being consolidated as the most promising technology to develop photonic devices for telecom applications with a very large scale of integration (VLSI) at a low cost [JAL06]. Besides its compatibility with Complementary Metal Oxide Semiconductor (CMOS) microelectronic tools, the high refractive index contrast between the core $\left(n_{S i} \approx 3.45\right)$ and cover $\left(n_{S i O 2} \approx 1.45\right)$ materials in SOI wafer layers results in a high light confinement in the core of optical structures. This confinement is key to the development of compact devices of reduced dimensions, and can thereby reduce the dimensions of integrated circuits, which is important since it is the basis to large-scale chip fabrication. Despite the fact that these structures exhibit unique characteristics that would present a radical advancement in technology today, the polarization control in such devices is in most cases complex, because of its inherent sensitivity to polarization [PAV04]. For this reason, the polarization sensitivity is a major problem in microphotonics, since the state of polarization changes randomly in the optical fibers, which involves an external control of the polarization in integrated photonic devices necessary to connect them to the outside world.

Regarding the $0^{\text {th }}$ order mode effective indices for each polarization $\left(n_{e f f, T E} \approx\right.$ $2.7, n_{\text {eff }, T M} \approx 1.6$ ), rectangular singlemode SOI waveguides have a very strong birefringence, and the mode strong confinement in the core occurs for Transverse Electric (TE) modes. For this reason, photonic devices are usually designed to operate with the TE polarization. As a result, integrated polarization diversity schemes have to be implemented when using rectangular SOI strip waveguides in order to avoid external polarization control and to make sure the waveguide is mostly guiding TE polarization [BAR07]. A schematic of a polarization diversity scheme is depicted in figure 3.1.

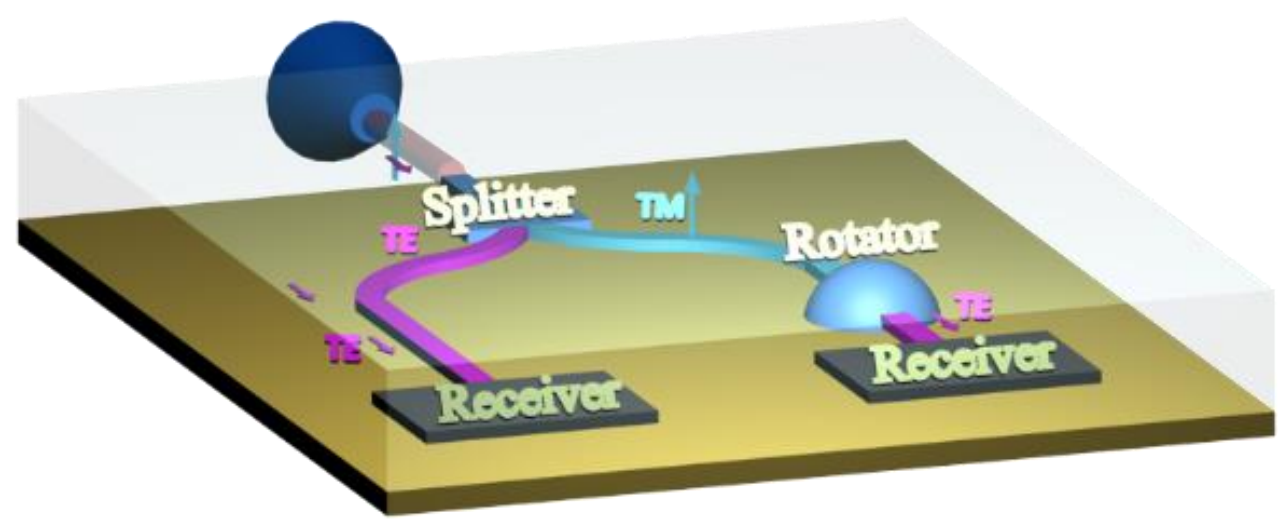

Figure 3.1: Polarization diversity scheme. 
As shown in the figure above, an arbitrary input polarization state coming from a fiber is split into orthogonally polarized components by a polarization splitter, so the TE and TM polarizations are completely separated and processed individually. These two components travel in separated arms, until the TM component reaches the polarization rotator, and is converted in a TE one to have the same polarization state at the input of the photonic structure, which is a DQPSK receiver in our case.

\subsubsection{Polarization Splitter}

The polarization state of light is, without doubt of great importance in telecommunications networks since many components of such networks are highly polarization dependent, and also because the polarization modal dispersion (PMD) can significantly degrade an optical fiber transmission. Hence, polarization splitters are one of the key elements in integrated optical circuits for routing and signal processing. A variety of polarization splitters has been reported to separate the random polarization coming from the input fiber, some based on directional couplers, such as [XIA08, TU10, WAN13], using Mach-Zehnder interferometers (MZIs) [LIA05b, DAI11a], photonic crystal (PhC) structures [SHI07] or multimode interference (MMI) structures [KAT09, YAN09]. The polarization splitter proposed by [XIA08] is based on a directional coupler in horizontal multiple-slotted waveguide structures. Despite the fact that the fabrication process steps increase, the device presents a large coupling length of about $238 \mu \mathrm{m}$. The simulations and results presented in [DAI11a] show that, if ridge waveguides are used, either MZI based polarization splitter and directional coupler based polarization splitter result in very long devices. The polarization splitter presented in [LAI05] confirms the theoretical study commented before, demonstrating that for a fabricated MZI acting as polarization splitter using rib waveguides, just the MZI arms are $6 \mathrm{~mm}$ long, a part of the MMIs needed at the input and output of the MZI, whose lengths are of about $2 \mathrm{~mm}$.

[KAT09] proposed a silicon slot waveguide polarization splitter using a MMI structure and presented theoretical and simulated studies, where the optimum polarization splitter length was shorter than $50 \mu \mathrm{m}$ with an extinction ratio (ER) near to $13 \mathrm{~dB}$, too small if compared with our ER target, as happens with the experimental demonstration of the polarization splitter based on MMI structure presented in [YAN09], where a compact device is achieved $(L=8.8 \mu \mathrm{m})$ but with ERs below $20 \mathrm{~dB}$, or a recent and compact polarization splitter based on an asymmetrical bent DC [WAN13] but that only achieves an ER of about 10dB.

The selected structure for our polarization splitter is a MMI based one since these structures are very compact, with a large optical bandwidth and high tolerances for fabrication deviations. The operation of a MMI device is relatively simple. The key component is a multimode waveguide that supports several modes (typically $\geq 3$ ). Its operating principle is the self-image principle and can be stated as follows: self-imaging is a property of multimode waveguides by which an input field profile is reproduced in single or multiple images at periodic intervals along the propagation direction of the 
guide, as shown in figure 3.2. For simplicity, only the formation of single and two-fold self-images is shown.

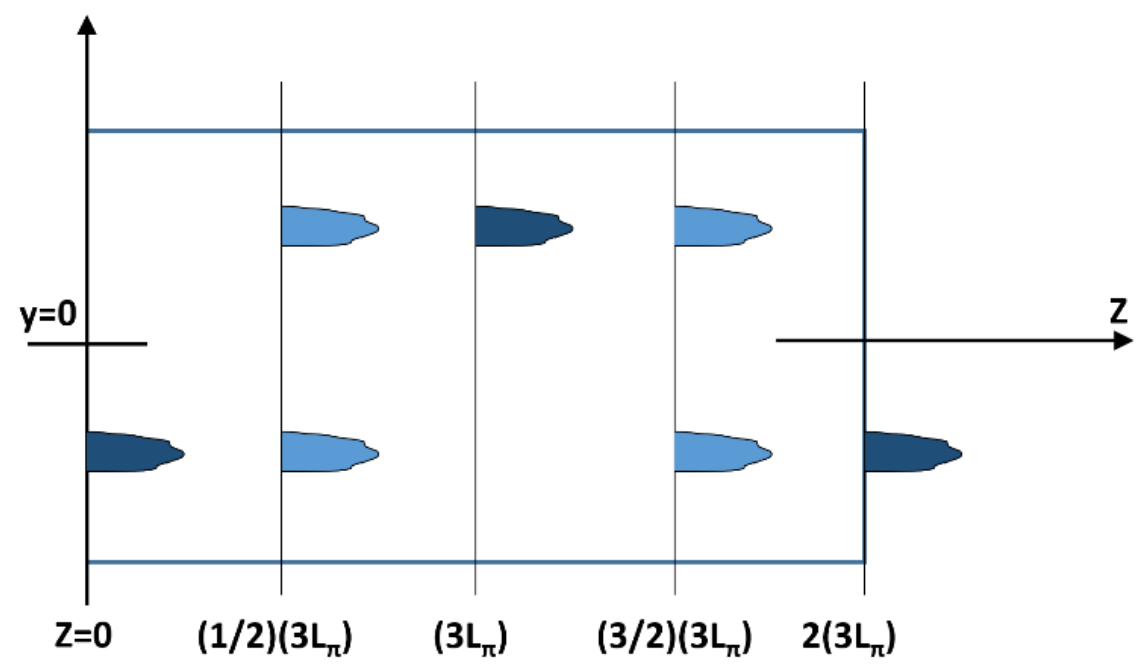

Figure 3.2: Self-image formation along a multimode waveguide showing single and two-fold images.

In the case of general interference, the formation of the self-images (single and multiple) along the multimode waveguide is given by:

$$
L=\frac{p}{N}\left(3 L_{\pi}\right)
$$

where $p \geq 0$ and $N \geq 1$ are integers with no common divisor [SOL95]. Here $N$ is related to the number of self-images and $p$ denotes the periodic nature of the imaging along the multimode waveguide for each set of $N$ images. In this case, $L_{\pi}$ is defined as the beat length between the two lowest order modes

$$
L_{\pi}=\frac{\pi}{\beta_{0}-\beta_{1}}
$$

where $\beta_{0}$ and $\beta_{1}$ are the propagation constants of the two lowest order modes.

As commented before, a MMI based polarization splitter was studied, but the obtained results did not achieve the required ER target. Consequently, a second design using a MM structure was carried out, but with the important restriction that only the two first modes will be supported by the multimode waveguide, for each polarization. This restriction was achieved by setting the width of the multimode waveguide as twice of the width of the input waveguides, as shown in figure 3.3. 


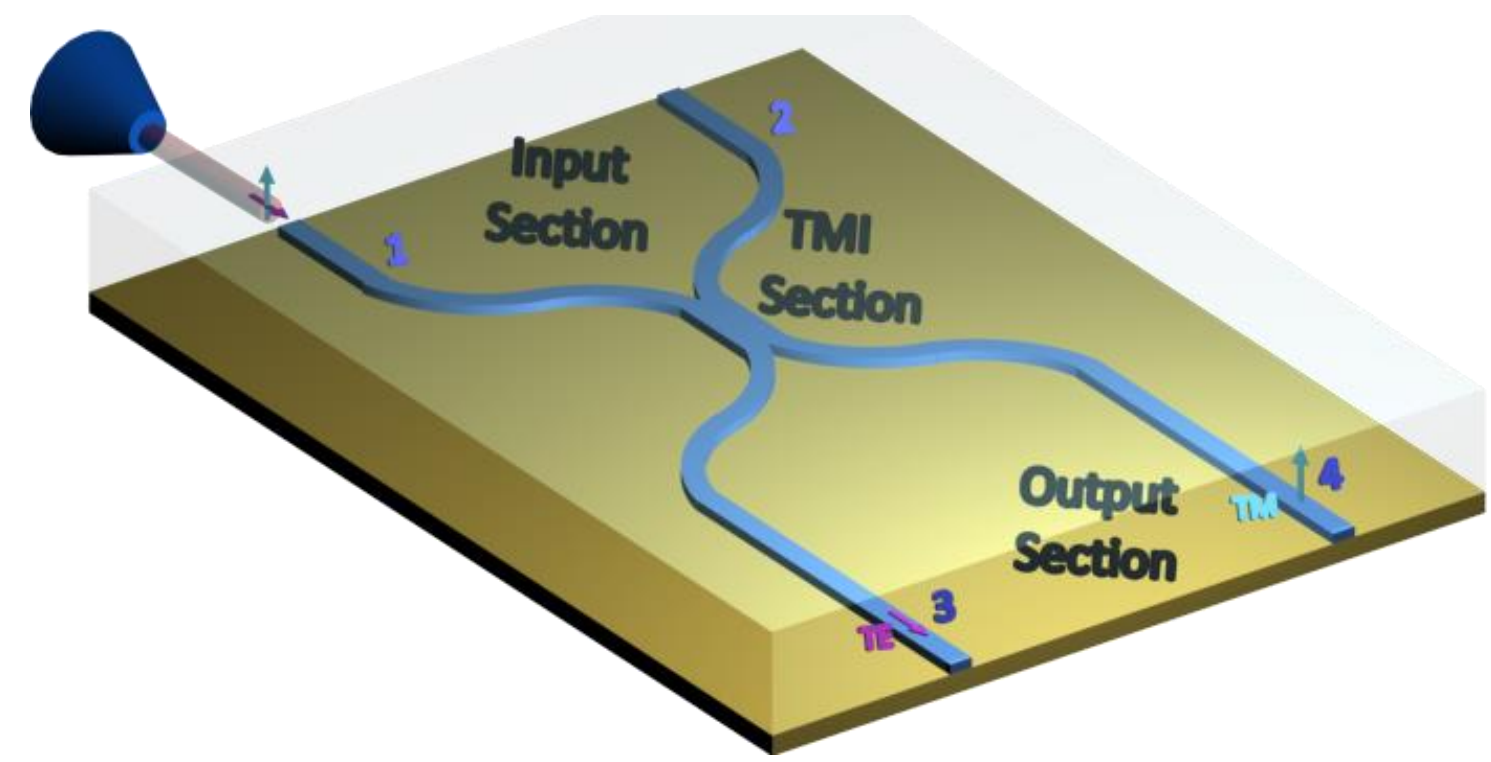

Figure 3.3: Proposed SOI polarization splitter.

It can be observed that the design contains three sections: the input and the output sections, formed by two single-mode SOI waveguides placed together so that there is no gap between them, and a central section, which we will refer to by TMI (Two-Mode Interference). This case presented here is a particular case of an MMI since we control that interference will occur only between two modes in the TMI section.

A linearly polarized input light at port 1 (decomposed in TE and TM polarizations) with power $P_{1}$ propagates through the input single-mode waveguide to the TMI section of the splitter. Then, it excites the $0^{\text {th }}$ and $1^{\text {st }}$ higher order modes of the corresponding polarization at the input plane of the TMI section. Output power in ports 3 and 4 due to two mode interference may be expressed as:

$$
\frac{P_{3}}{P_{1}}=\sin ^{2}\left(\frac{\pi L}{2 L_{c}}\right), \frac{P_{4}}{P_{1}}=\cos ^{2}\left(\frac{\pi L}{2 L_{c}}\right)
$$

where $L$ is the total TMI section length and $L_{c}$ is the fundamental coupling length for each polarization component $\left(L_{c}^{T E}=\lambda_{0} /\left(2 \cdot \Delta n_{e f f}^{T E}\right), L_{c}^{T M}=\lambda_{0} /\left(2 \cdot \Delta n_{e f f}^{T M}\right)\right.$, being $\lambda_{0}$ the vacuum wavelength, and $\Delta n_{\text {eff }}$ the effective index difference of $0^{\text {th }}$ and $1^{\text {st }}$ higher order modes of each polarization). Considering the equation below,

$$
\mathrm{L}_{\mathrm{TMI}}=\mathrm{m} \cdot L_{c}^{T M}=(\mathrm{m}+1) \cdot L_{c}^{T E}
$$

being $m$ an integer, the polarization splitter will be in a bar state if $m$ is an odd number, and in a cross state when $m$ is an even integer, for TE input polarization. So by choosing the optimum $\mathrm{L}_{\mathrm{TMI}}$, bar/cross state of the device will split the polarization properly.

For SOI wafers with $220 \mathrm{~nm}$ thick Si layer and a $900 \mathrm{~nm}$ wide TMI waveguide section, we first calculated $L_{c}$ for TE and TM polarizations at $\lambda=1550 \mathrm{~nm}$. We obtained $\mathrm{L}_{\mathrm{c}}^{\mathrm{TE}}=$ $1.93 \mu \mathrm{m}$, and $\mathrm{L}_{\mathrm{c}}^{\mathrm{TM}}=2.25 \mu \mathrm{m}$ using a full-vectorial mode solver for calculating all 
effective indices involved. With these values, we obtained that $\mathrm{L}_{c}^{\mathrm{TM}} / \mathrm{L}_{c}^{\mathrm{TE}}=1.166=7 / 6$, which corresponds to $m=6$, and thus, the optimal TMI section length is $\mathrm{L}_{\mathrm{TMI}}=13.5 \mu \mathrm{m}$.

Figure 3.4 depicts the theoretical normalized output power at ports 3 and 4 as a function of $\mathrm{L}_{\mathrm{TMI}}$ for both TE and TM polarizations at the operating wavelength of $\lambda=1550 \mathrm{~nm}$.

a)

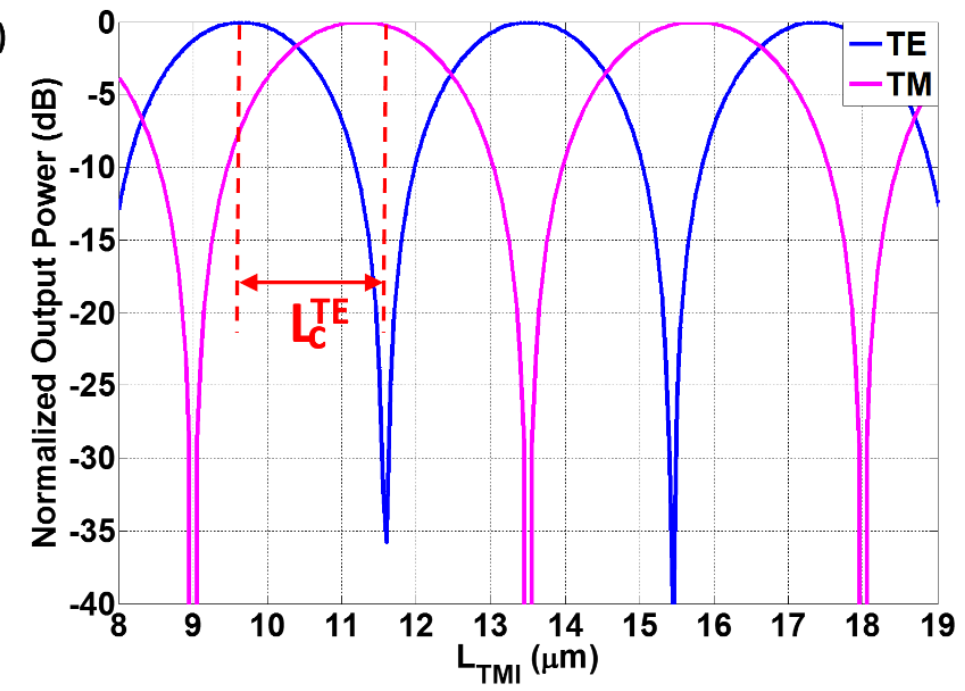

b)

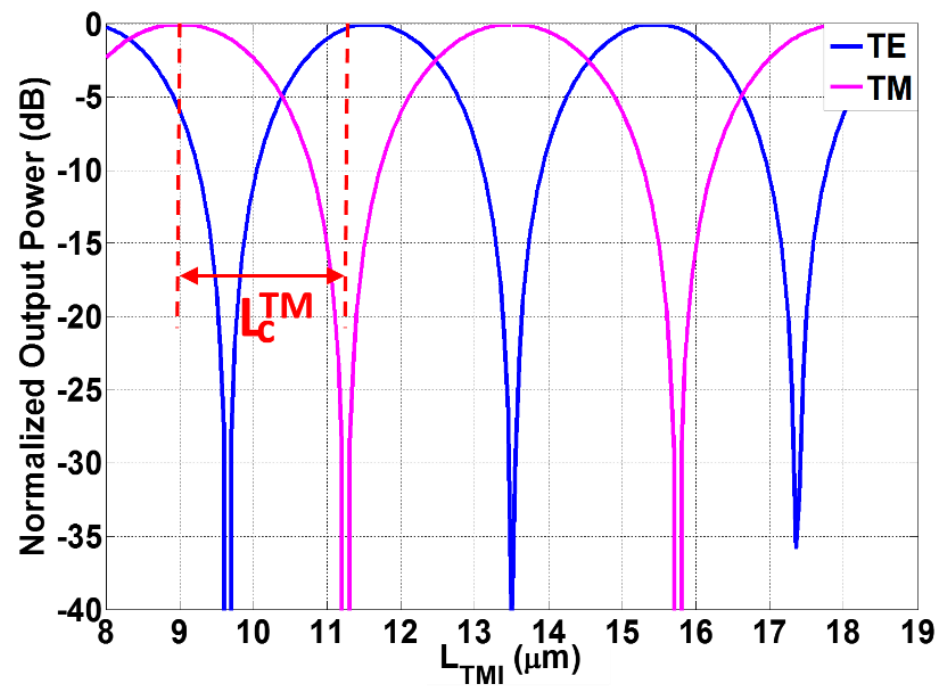

Figure 3.4: Theoretical normalized output power at a) port 3 and b) port 4 for TE and TM polarizations as a function of $\mathrm{L}_{\mathrm{TMI}}$ for $\lambda=1550 \mathrm{~nm}$

It can be seen that if we choose a $13.5 \mu \mathrm{m}$ long TMI section, polarization is splitted as expected. The figure above also illustrates the value of $L_{c}$ for each polarization according to simulation results. The figure below shows a simulation capture using the $3 \mathrm{D}$-finitedifference-time-domain method (3D-FDTD), which verifies that with the selected length, $\mathrm{L}_{\mathrm{TMI}}=13.5 \mu \mathrm{m}$, the separation of the two polarizations is achieved. Moreover, we can also observe 7 images for TE polarization $\left(7 \cdot \mathrm{L}_{\mathrm{c}}^{\mathrm{TE}}\right)$ and 6 images for TM polarization $\left(6 \cdot \mathrm{L}_{\mathrm{c}}^{\mathrm{TM}}\right)$ within the TMI section, according to the teoretical equation described by Eq. 3.4 . 
TE in

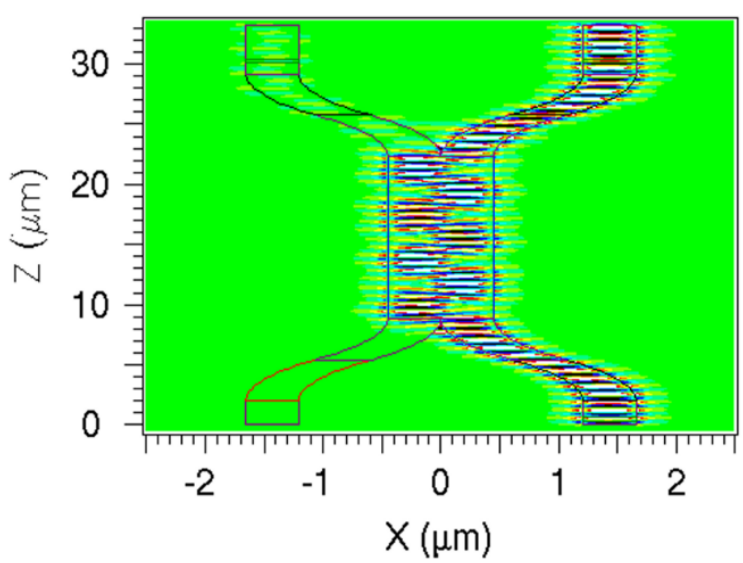

TM in

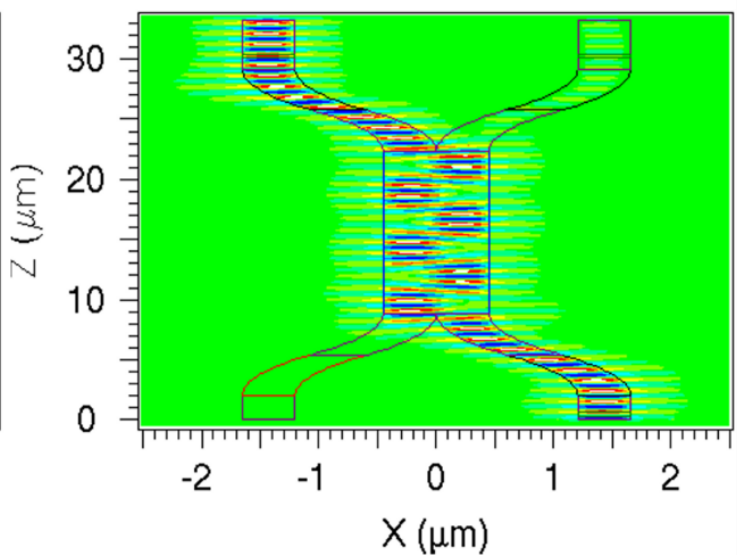

Figure 3.5: 3D-FDTD simulation capture for an input (Left) TE, and (Right) TM, polarization.

Once the theoretical studies and simulations are done, the fabrication of the device is carried out. Polarization splitters with different TMI section lengths with the same aspect than depicted in Figure 3.3 were fabricated. Curved waveguides of $10 \mu \mathrm{m}$ radius were used as waveguide ports of the splitter.

The fabrication process of the polarization splitter was carried out at Nanophotonics Technology Center's (NTC) facilities by using e-beam lithography over Hydrogen SilsesQuioxane (HSQ) negative resist. The electron dose was adjusted for achieving the optimized dimensions. After developing the sample, the patterned resist was employed as a mask in the following fabrication step consisting of a dry etching by using an Inductive Coupled Plasma (ICP) system. Figure 3.6 depicts scanning electron microscope (SEM) images of the fabricated devices (Figure. 3.6 (a)) as well as a detailed top view of the coupling section of the splitter (Figure. 3.6 (b)).
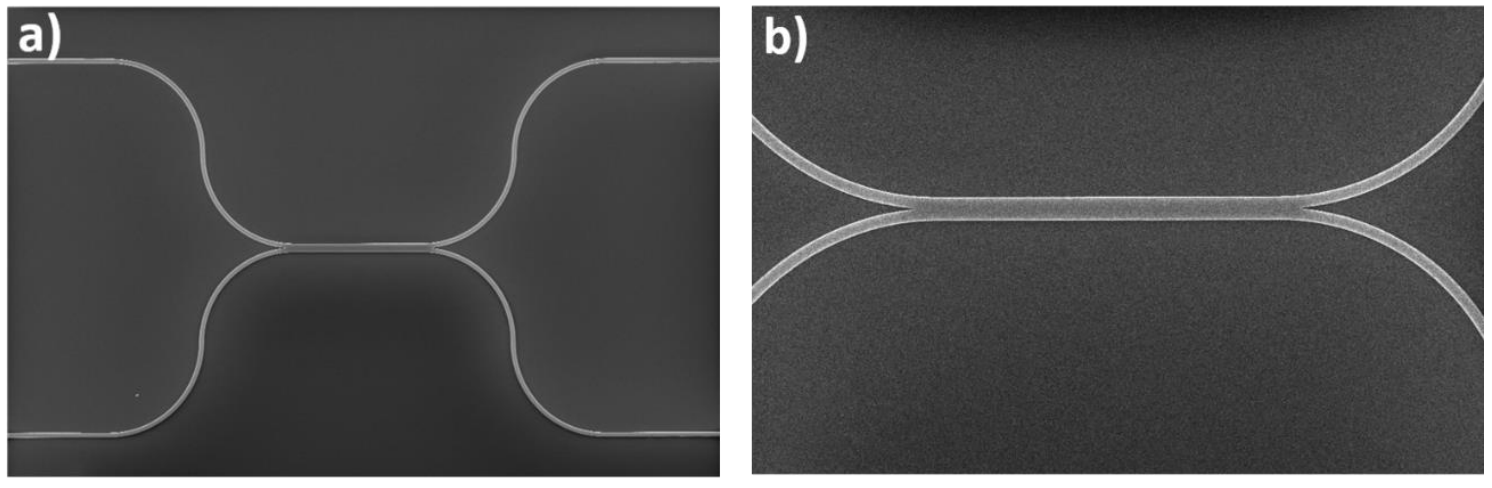

Figure 3.6: SEM images of a) fabricated polarization splitter and b) detail of the coupling section.

For the experimental characterization of the fabricated devices, the setup shown in figure 3.7 was used. The different components of the setup of Figure 3.7 are illustrated in the following block diagram depicted in Figure 3.8. 


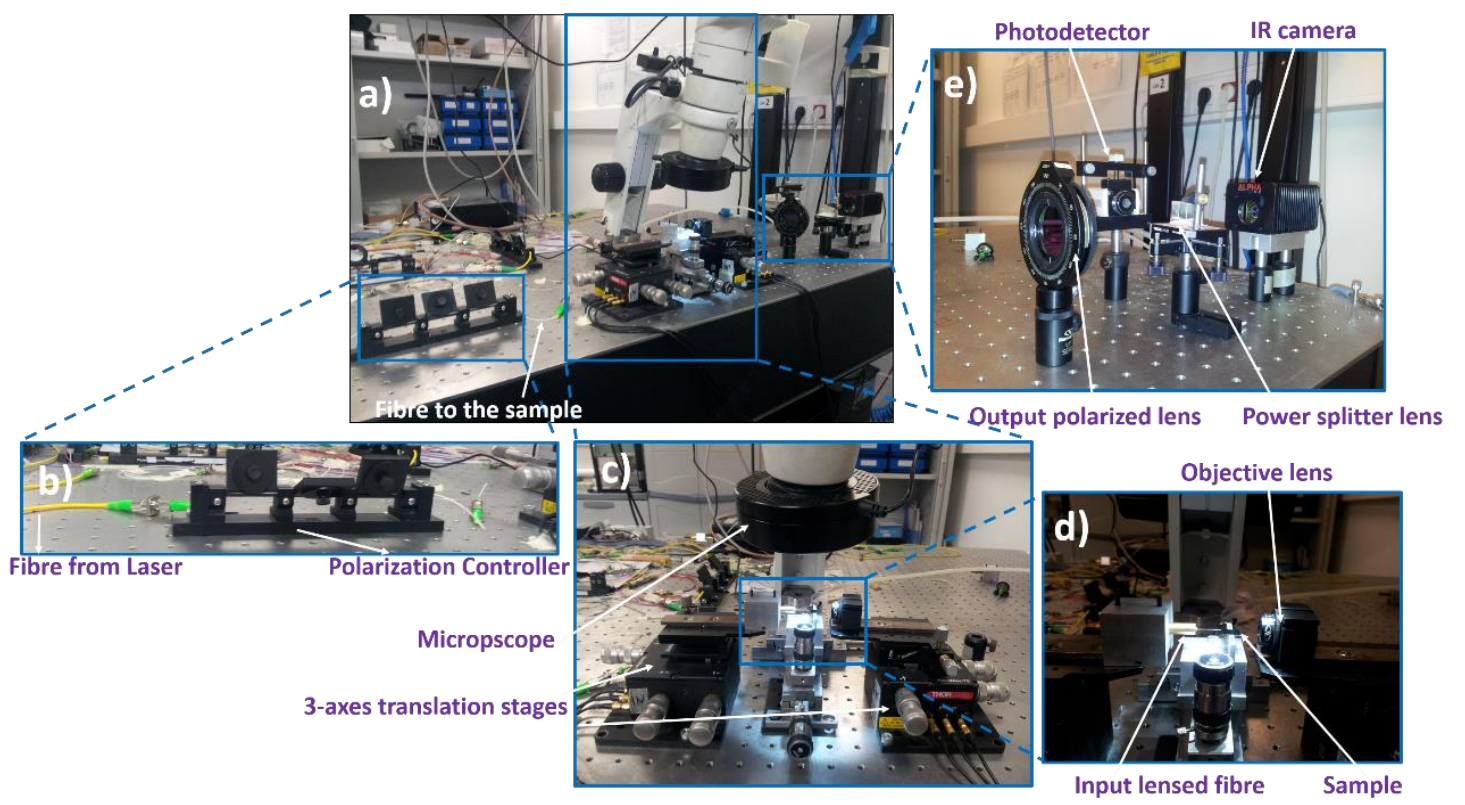

Figure 3.7: (a) Image of the whole experimental set-up used for the polarization splitter characterization. (b) Fiber coming from laser to input PC. (c) Translation stages and microscope. (d) Input lensed fiber, sample and objective lens. (e) Output polarized lens, power splitter lens, photodetector and IR camera.

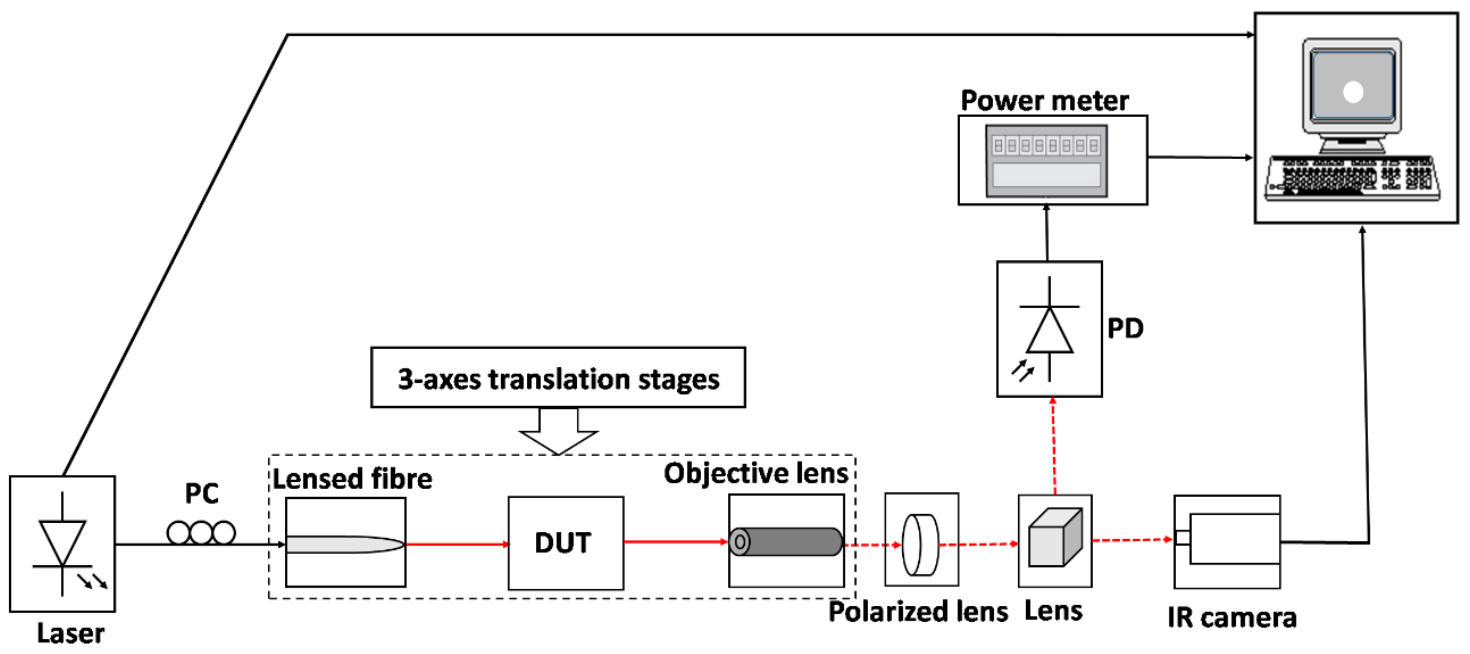

Figure 3.8: Block diagram of the setup for polarization splitter experimental measurements.

As excitation source, a broadband laser (SANTEC TSL-210F) was used. After the laser, a polarization controller (PC) was placed. Because the device under study has to separate the two polarizations, we must control the polarization of the light incident upon it in order to check its correct performance. After the polarizer, the light is injected into the chip with the selected polarization, through a lensed fiber. With the output polarizer lens, the polarization to be measured can be selected, being in a horizontal position for measuring the TE polarization and in a vertical position for the TM one. Next we have placed a lens that splits the received light to a power meter (Q2140 ANDO) and to an infrared camera (IR camera in Figure. 3.8), which were connected to a computer by a GPIB bus, in order to visualize the light spot (see Figure 3.9 (a)) and measure the received power at the same time. The 3 -axes translation stages are connected to a piezoelectric 
controller, which is depicted in Figure 3.9 (b) so a finer adjustment in alignment process between the fiber, the sample and the objective lens may be done.

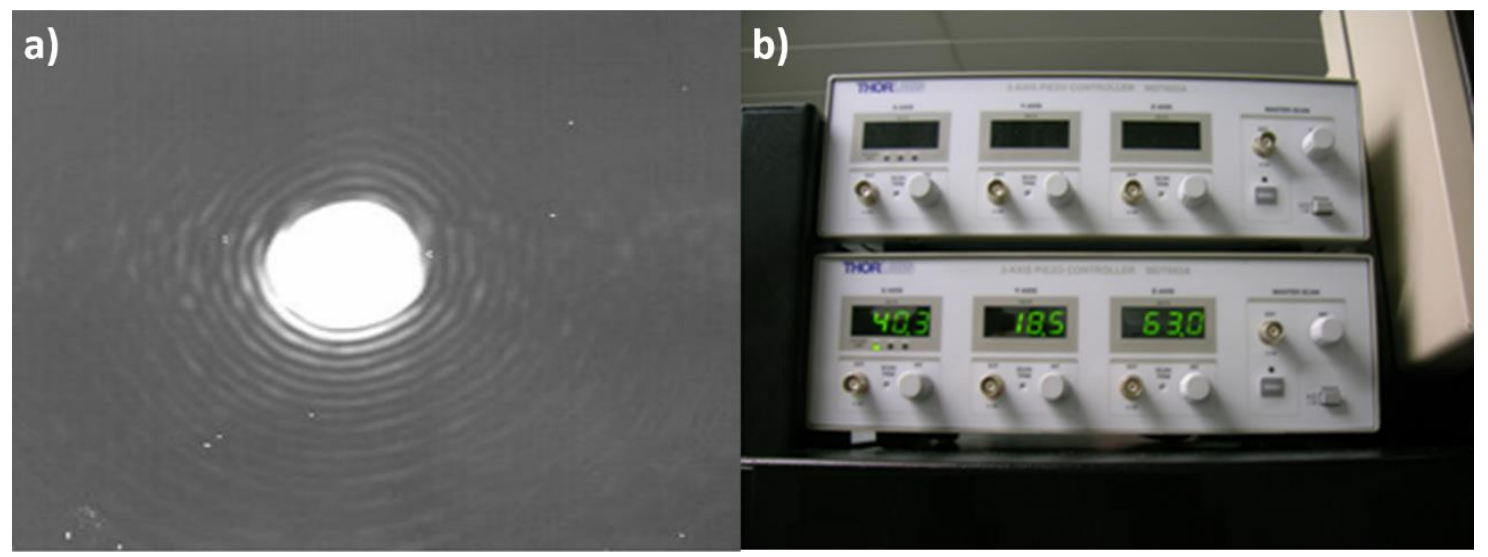

Figure 3.9: (a) Light spot captured by the infrared camera after proper alignment between the fiber, sample and objective lens. (b) Piezoelectric controller.

Once the alignment between the fiber, sample and objective lens was done, we proceeded to characterize the polarization splitter performance, taking into account that the losses introduced by the setup are of about $8 \mathrm{~dB}$. As commented before, polarization splitter with different TMI section length were fabricated, and measured. The figure below, Figure 3.10, shows the theoretical normalized output powers and the measured ER of the eight fabricated devices, for both TE and TM polarizations, at $\lambda=1550 \mathrm{~nm}$.

As it can be seen, the splitter that provides the best performance in terms of ER is the $13.5 \mu \mathrm{m}$-long, with a measured insertion loss ranging between 2 and $3 \mathrm{~dB}$. Figure 3.11(a) illustrates the experimental measurements, corresponding to the selected polarization splitter, of the output power at port 3(bar port), while Figure 3.11(b) shows the power measurements corresponding to the output port 4 (cross port), both containing TE and TM polarization graphs.

As depicted in Figure 3.10 and in Figure 3.11, for the bar port, we measured an ER of $20.1 \mathrm{~dB}$, while the obtained ER for the cross port was about $22.15 \mathrm{~dB}$, for the operating wavelength $\lambda=1550 \mathrm{~nm}$. The measured values have the same trend as expected from theoretical and simulated results. Furthermore, as shown in Figure 3.11, measured ER remains almost constant in a wavelength bandwidth of about $40 \mathrm{~nm}$. 

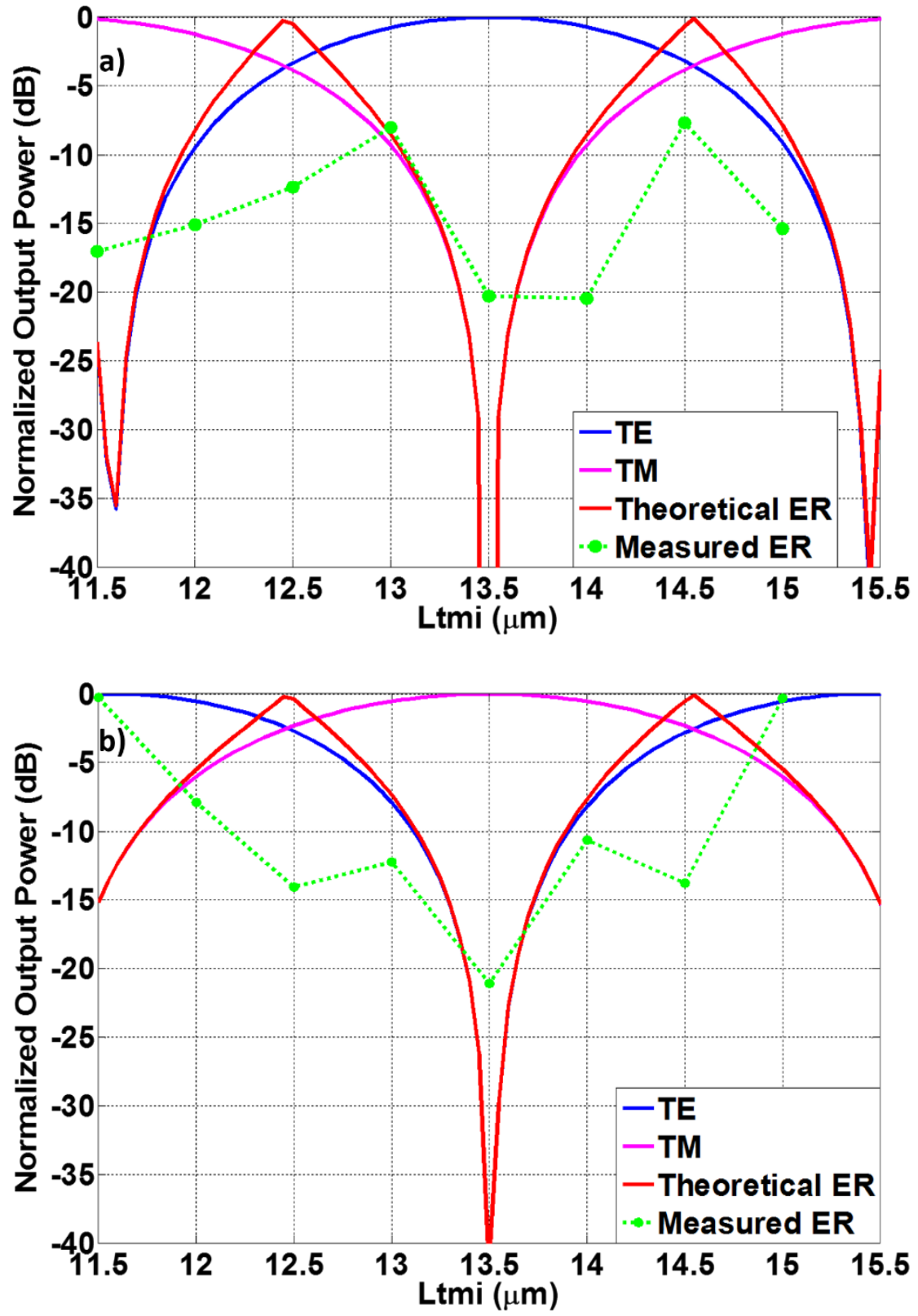

Figure 3.10: Normalized output power and theoretical and experimental extinction ratio for fabricated devices, for (a) bar and (b) cross ports.

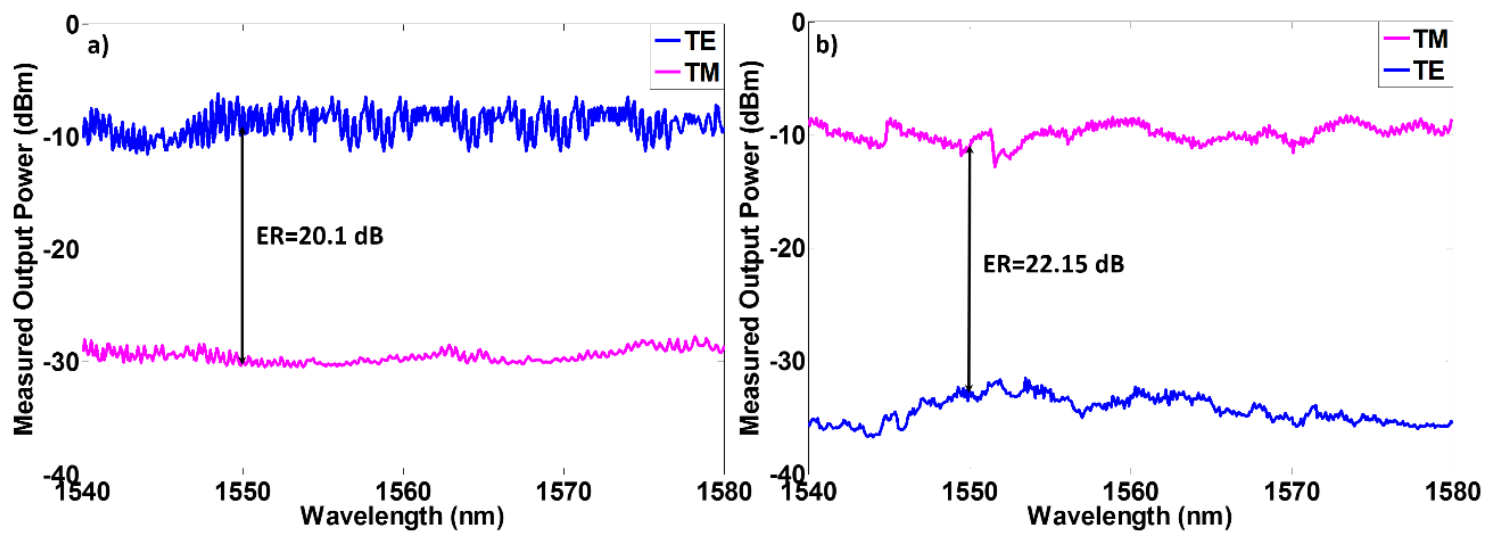

Figure 3.11: Measured output power spectrum at a) bar and b) cross ports. 


\subsubsection{Polarization Rotator}

Several approaches have been proposed to rotate the polarization in silicon waveguides. Mode evolution based rotation has been demonstrated by twisting the waveguide using two asymmetric layers oppositely tapered [ZHA10b]. However in this case the input and output silicon waveguides have different thicknesses imposing a serious restriction when integrated in a more complex device. Alternatively, an asymmetrical directional coupler has been demonstrated which only requires one lithography and etching step [LIU11a] and that can also be used for splitting the polarization [DAI11b, LIU11b]. However, longer lengths are required and the vertical symmetry of the coupler must also be broken by using a different top-cladding material from that of the buffer layer which again would impose restrictions for its integration. In this way, the use of different materials on top of single silicon waveguides have also been demonstrated for achieving polarization rotation [FUK08,CHE11].

A pure silicon solution without the need of extra materials can be achieved by breaking the symmetry of the waveguide cross section. Hence, the propagation modes are hybridized allowing power to be transferred periodically between the two desired polarization states. Initially, angled waveguides were demonstrated but requiring complicated fabrication processes [BRO06]. Therefore, a simple two-step etching to build a stair-like cross section was proposed which in addition shortened the rotation length [WAN08]. Multiple subwavelength trenches [VEL12] or adding a polycrystalline silicon overlay [VER12] have been proposed and demonstrated to minimize the rotation lengths but once more at the expense of increasing the number of fabrication steps.

The proposed polarization rotator, designed especially to be compatible with standard SOI wafers with $220 \mathrm{~nm}$ thick silicon layer and its associated standard grating couplers for efficient coupling to external optical fibers [TAI02], consists of a straight asymmetric structure shallow etched in a strip waveguide configuration as depicted in Figure 3.12.

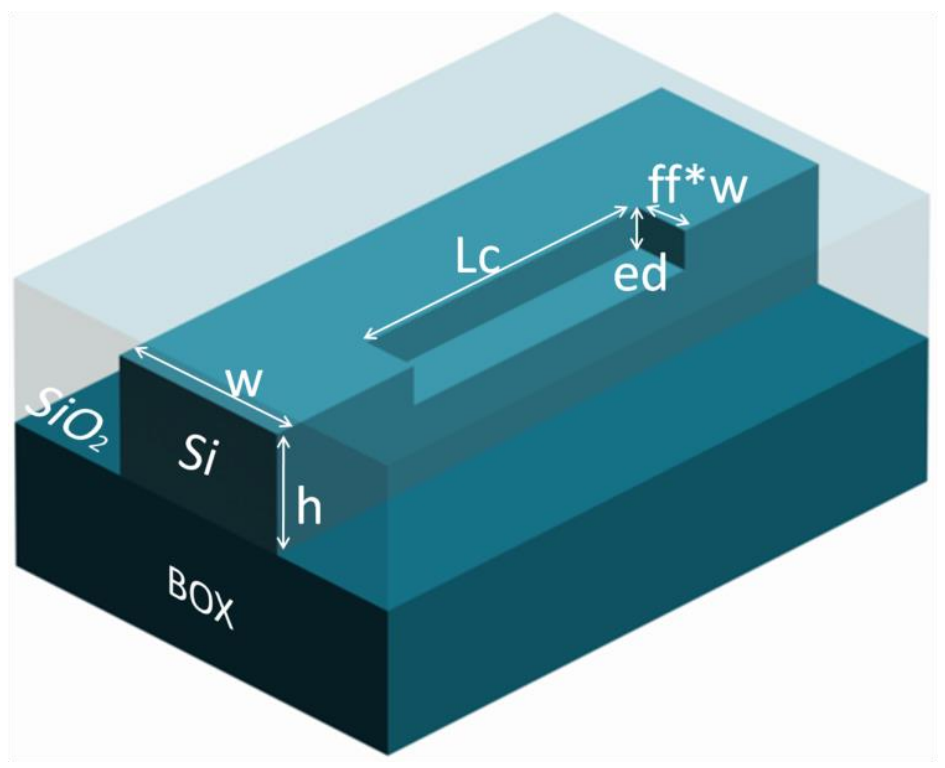

Figure 3.12: Proposed polarization rotator structure 
The chosen parameters are $h=220 \mathrm{~nm}$ (silicon thickness in standard SOI wafers), etch depth, $e d=70 \mathrm{~nm}$, and fill factor, $f f$, of $25 \%$. The etch depth was chosen to facilitate the integration of the polarization rotator in devices that use conventional gratings to couple light from an external optical fiber [TAI02].

An asymmetric waveguide structure allows the generation of two fundamental polarization modes that are sufficiently hybrid. To possess the polarization conversion behaviour, the asymmetric waveguide must have two modes with almost the same intensity distributions, and their optical axes are perpendicular to each other.

A symmetric-asymmetric waveguide interface allows the excitation of two hybrid modes $S_{1}$ and $S_{2}$ with their principal axes tilted by 45 degrees, as shown in the figure below (Figure 3.13(a)).

a)
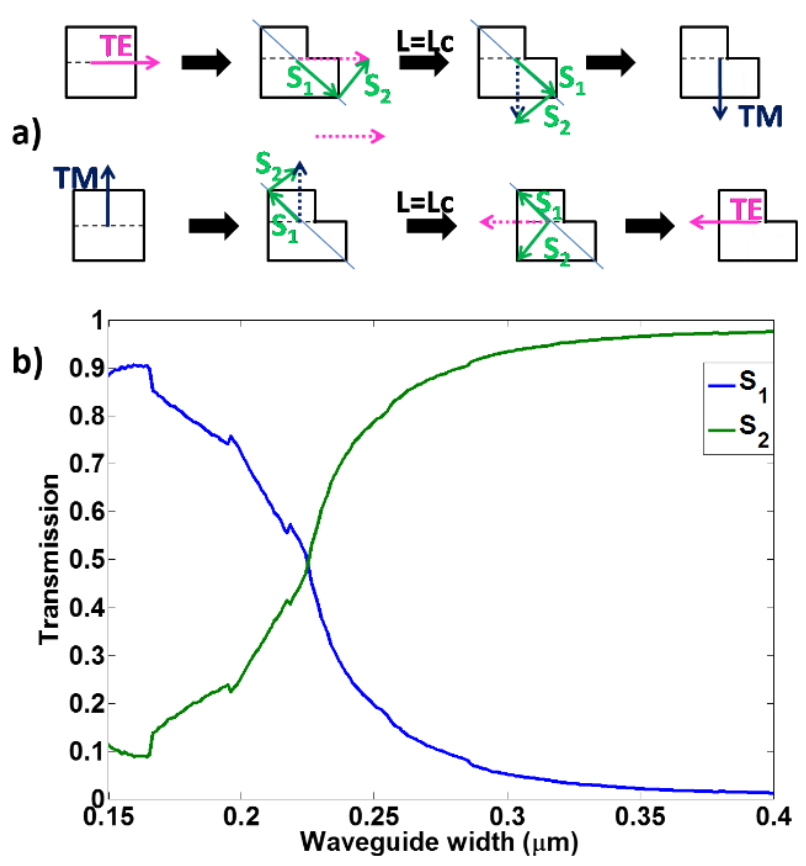

Figure 3.13: (a) Optical axes rotation after propagating along Lc. (b) simulated excitation of hybrid modes as a function of the waveguide width of the proposed polarization rotator, at the symmetric-asymmetric interface.

In order to achieve total polarization conversion, these hybrid modes must be excited equally. When an input polarization propagates along the polarization rotator during $L_{c}$, one of the optical axes suffers a rotation of 180 degrees, resulting in the orthogonal polarization at the output of the polarization rotator section, as depicted in Figure 3.13(a). This condition is satisfied for a waveguide width of around $w=220 \mathrm{~nm}$, as shown in Figure 3.13(b).

The polarization rotators are usually characterized by measuring the TE to TM transmission, normalized with the reference measurements of the grating couplers. The TE to TM coupling efficiency, which is the key characteristic of the proposed device, has to be maximized for optimum performance. Therefore, the polarization conversion 
efficiency (PCE), which is a measure of the extinction ratio at the output port, has been defined as:

$$
P C E(d B)=10 \log _{10}\left(\frac{T_{T E-T M}}{T_{T E-T E}+T_{T E-T M}}\right)
$$

where $T_{T E-T M}$ is the power coupled to TM polarization when the input polarization is TE while $T_{T E-T E}$ is the power of the undesired non-rotated TE polarization for the same case.

Once all the parameters of our polarization rotator are defined, simulations and analysis were carried out by using a fully vectorial and bi-directional optical propagation tool based on eigenmode expansion [PHO12] in order to obtain $L_{c}$ so PCE is maximized. Figure 3.14 shows the PCE as a function of the rotator length $L_{c}$, where it can be concluded that for the optimal theoretical length value $(\sim 23 \mu \mathrm{m})$, the PCE is almost perfect.

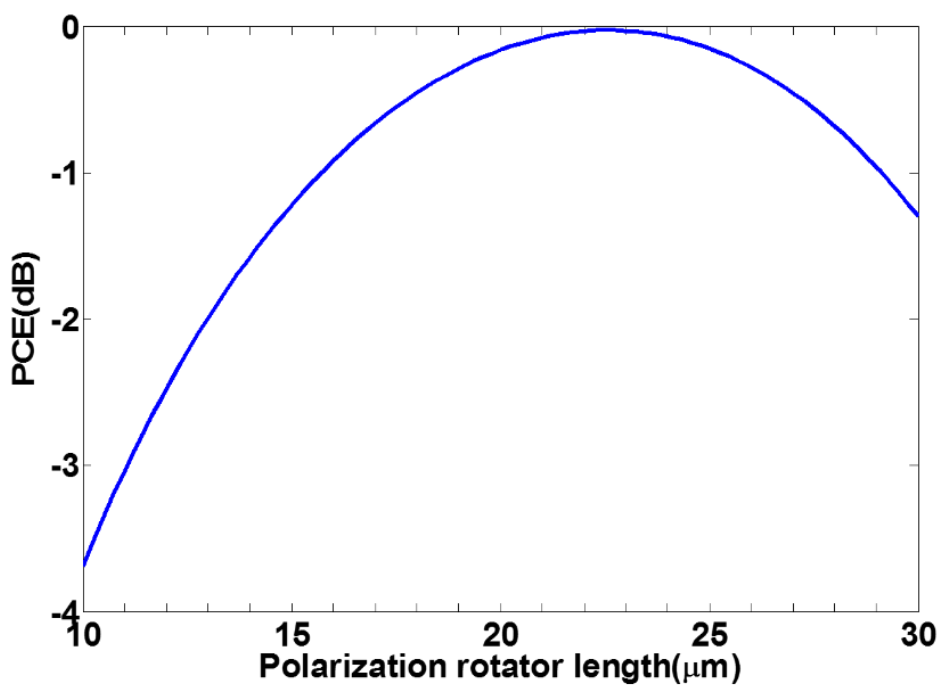

Figure 3.14: Polarization conversion efficiency (PCE) as a function of the polarization rotator length for the optimum waveguide width of $220 \mathrm{~nm}$.

The robustness of the proposed device was also studied by scanning the critical parameter space of the polarization rotator where the length $L_{c}$ is kept constant. The cross in the robustness figure indicates the zero penalization in the PCE, because it is the optimal obtained from simulations value. In Figure 3.15 the PCE penalty is plotted versus the fill factor $f f$ and etch depth ed deviations. The fill-factor, and therefore the mask alignment deviation, $-0.6 \mathrm{~dB}$ PCE tolerance is $+18 \mathrm{~nm}$ and $-20 \mathrm{~nm}$. For a $-0.6 \mathrm{~dB}$ PCE penalty, the etch depth may vary $\pm 18 \mathrm{~nm}$. Note that the etch depth is fixed in our case by means of a $\mathrm{SiO}_{2}$ etch stop layer, as it will be explained in the next paragraph, which will improve the overall robustness of our component. 


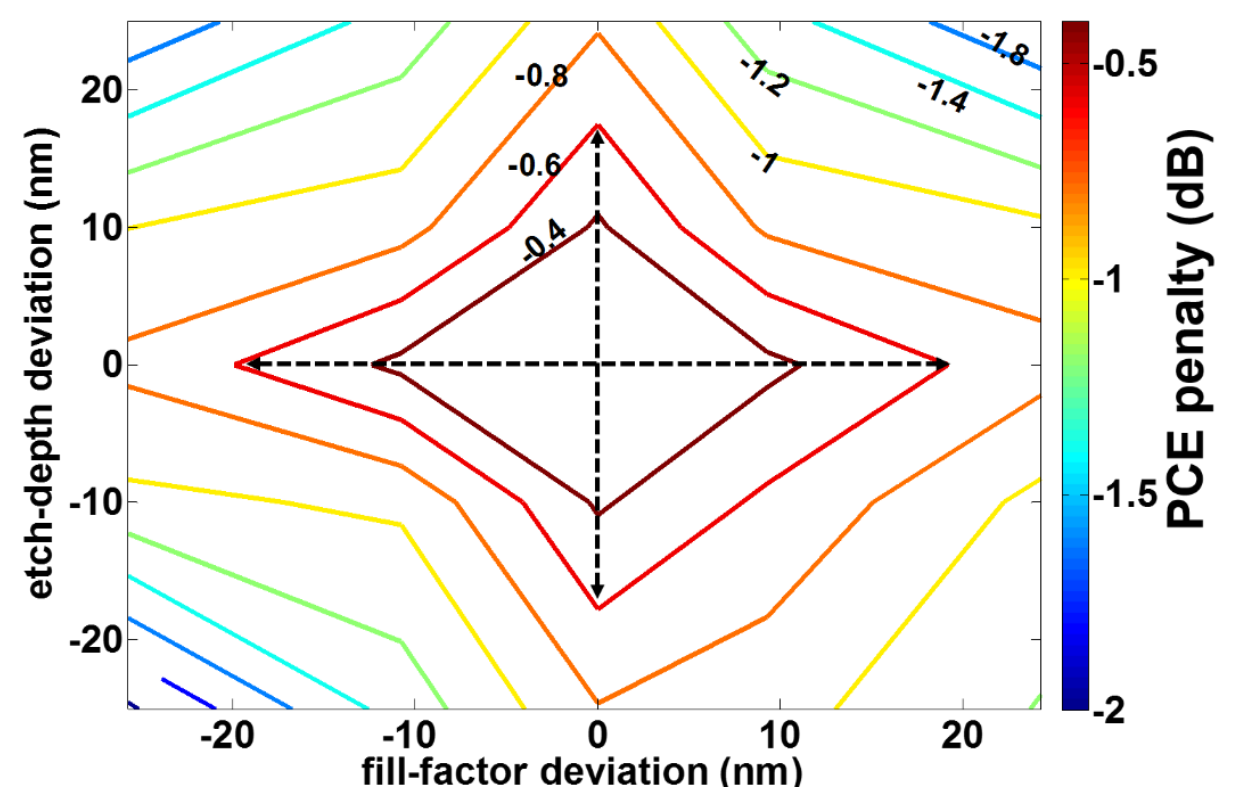

Figure 3.15: Polarization rotator robustness investigation as a function of the fill factor and etch depth. The contour lines are the PCE penalties with respect to the highest PCE of the device.

The complete CMOS fabrication process was carried out at Laboratory of Electronics, Technology and Instrumentation (LETI) facilities by using 193nm deep UV lithography. It starts with 100nm high temperature oxide (HTO) on top of a SOITEC wafer consisting of a $220 \mathrm{~nm}$ thick silicon epilayer on top of a $2 \mu \mathrm{m}$ thick buried oxide (BOX). The gratings and the waveguide arms are first patterned, followed by reactive ion etching (RIE) silica with $\mathrm{C}_{4} \mathrm{~F}_{8}$, which defines a hardmask. The silicon is then partially etched with $\mathrm{HBr}$ and controlled by ellipsometry in order to define precisely the grating teeth and the polarization rotator etch depth. In the second lithography step, the gratings and the polarization rotator are protected by the resist and the remaining hardmask serves for the waveguides in a self-alignment process. Then a full silicon etch down to the buffer silica layer completes the waveguide fabrication. Finally, a top silica cladding was deposited achieving around $1 \mu \mathrm{m}$ thickness.

Figure 3.16(a) shows a schematic of the whole polarization diversity structure used to characterize the fabricated polarization rotator, with SEM images of the different parts of the circuit. As it can be seen, the first element is a TE focusing grating coupler with a period of $630 \mathrm{~nm}$ and a coupling efficiency of around $-5 \mathrm{~dB}$. Figure 3.16(b) shows a SEM image of the TE focusing grating. The next structure the TE polarized light propagates along is the polarization rotator, with the parameters previously designed. A top SEM image of the fabricated rotator is depicted in Figure 3.16(c). At this point, the TE and TM modes are separated by a $13 \mu \mathrm{m}$-length directional coupler, shown in Figure 3.16(d), with a gap of $300 \mathrm{~nm}$, acting as a polarization splitter with a measured extinction ratio above $25 \mathrm{~dB}$. Once the two polarizations are separated, the TE and TM modes travel along the splitter output arms, to be recollected by the output fiber, through the TE and TM output grating couplers, respectively. The TM output grating, with a period of $1 \mu \mathrm{m}$, is depicted in Figure 3.16(e), and has a coupling efficiency of around $-6 \mathrm{~dB}$. 


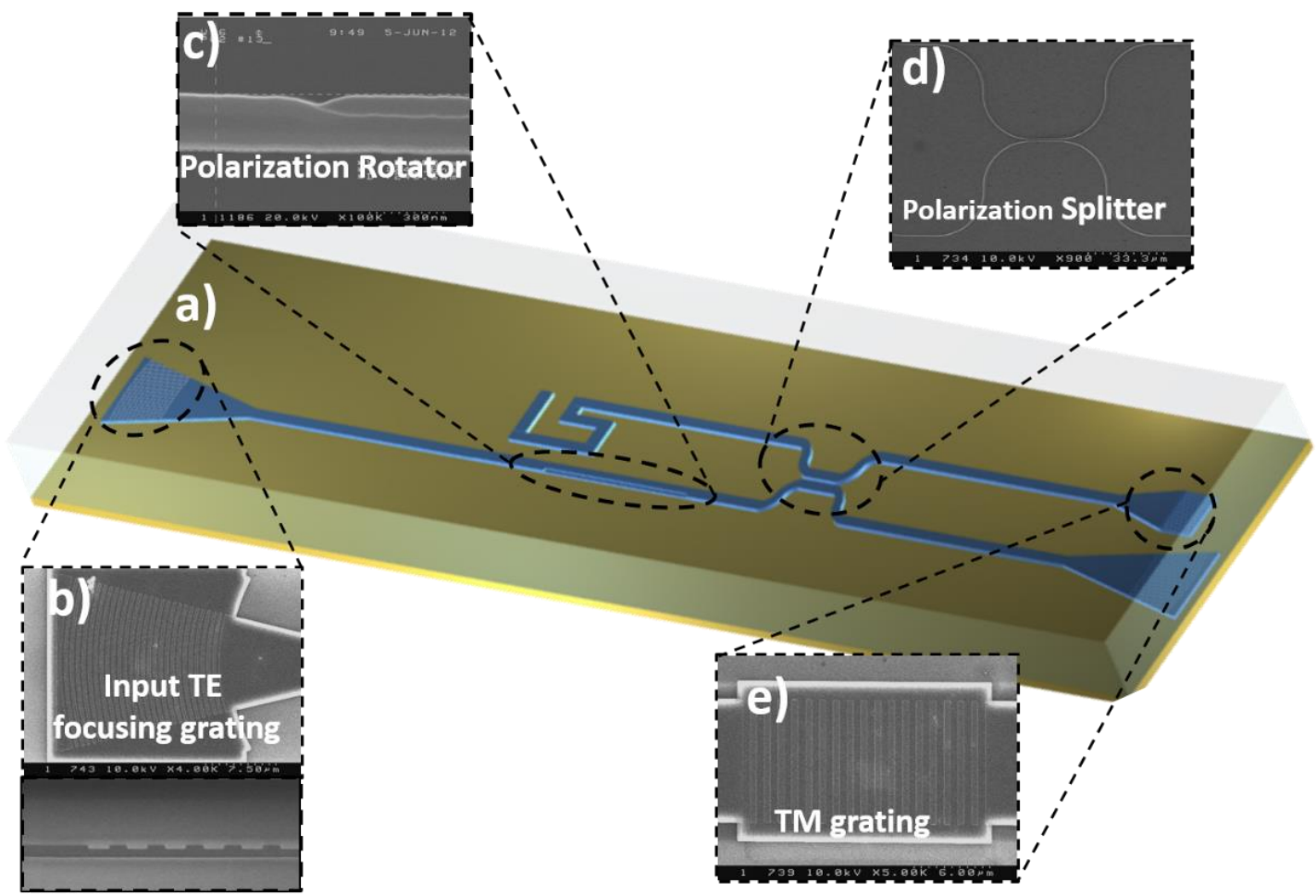

Figure 3.16: (a) Schematic of the whole polarization diversity structure used to characterize the polarization rotator. SEM images of (b) TE input focusing grating coupler, (c) polarization rotator, (d) polarization splitter and (e) TM output grating coupler.

Polarization rotators with different lengths were fabricated and characterized using the setup illustrated in Figure 3.17.

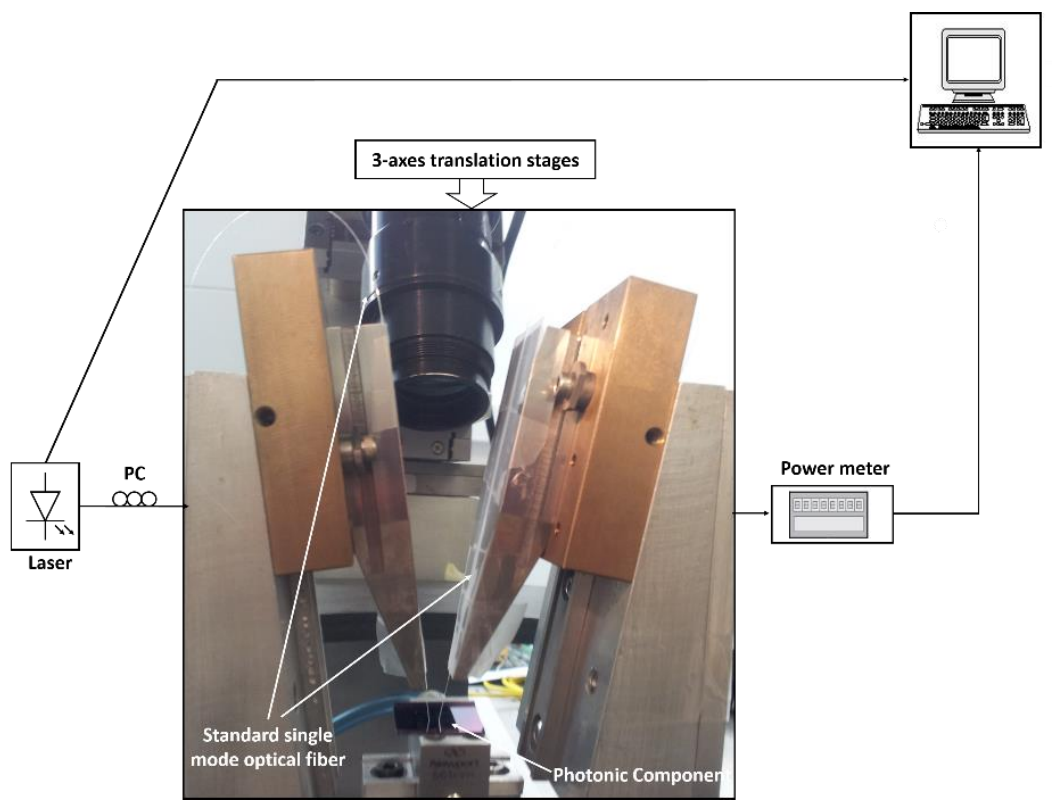

Figure 3.17: Block diagram of the setup for polarization rotator experimental measurements.

Measurements were performed with a single mode fiber tilted under an angle of 13 degrees relative to the surface normal of the grating. The input polarization state was firstly set by adjusting an external polarization controller and monitoring the output power measured in a reference straight waveguide. The efficiency of the rotator was thus 
measured by comparing the TE and TM polarization powers after coupling a TE polarization to the circuit, without any residual TM polarization due to the filtering effect produced by the input grating coupler, then passing through the rotator and finally using the polarization splitter to separate the rotated TM mode from the undesired non-rotated TE one.

The figure below, Figure 3.18, shows the normalized measured spectra of the polarization diversity structure shown in Figure 3.16 at the two outputs, namely $\mathrm{T}_{\mathrm{TE}-\mathrm{TM}}$ and $\mathrm{T}_{\mathrm{TE}-\mathrm{TE}}$, for three different lengths of the polarization rotator. The normalization was carried out taking into account the input and output gratings as well as the polarization splitter. As it can be seen, the insertion loss is ranging from $-1 \mathrm{~dB}$ to $-2.5 \mathrm{~dB}$ for the different characterized devices.

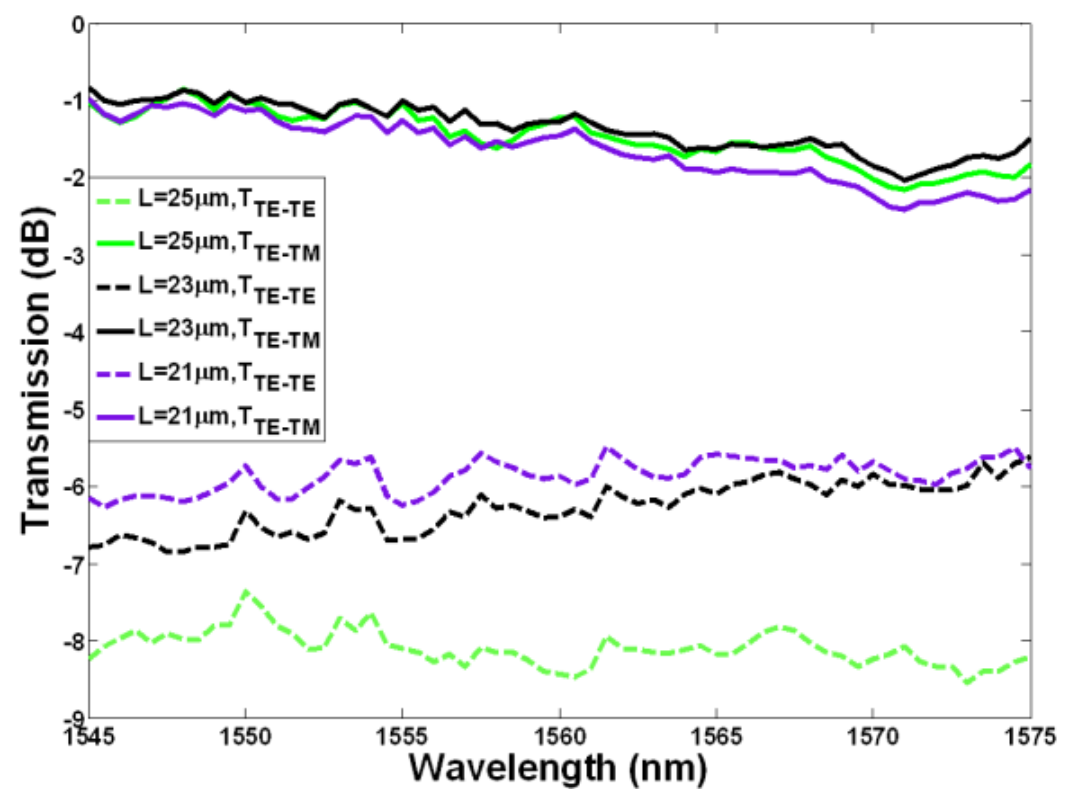

Figure 3.18: Normalized measured spectra as a function of wavelength of polarization rotators with three different lengths.

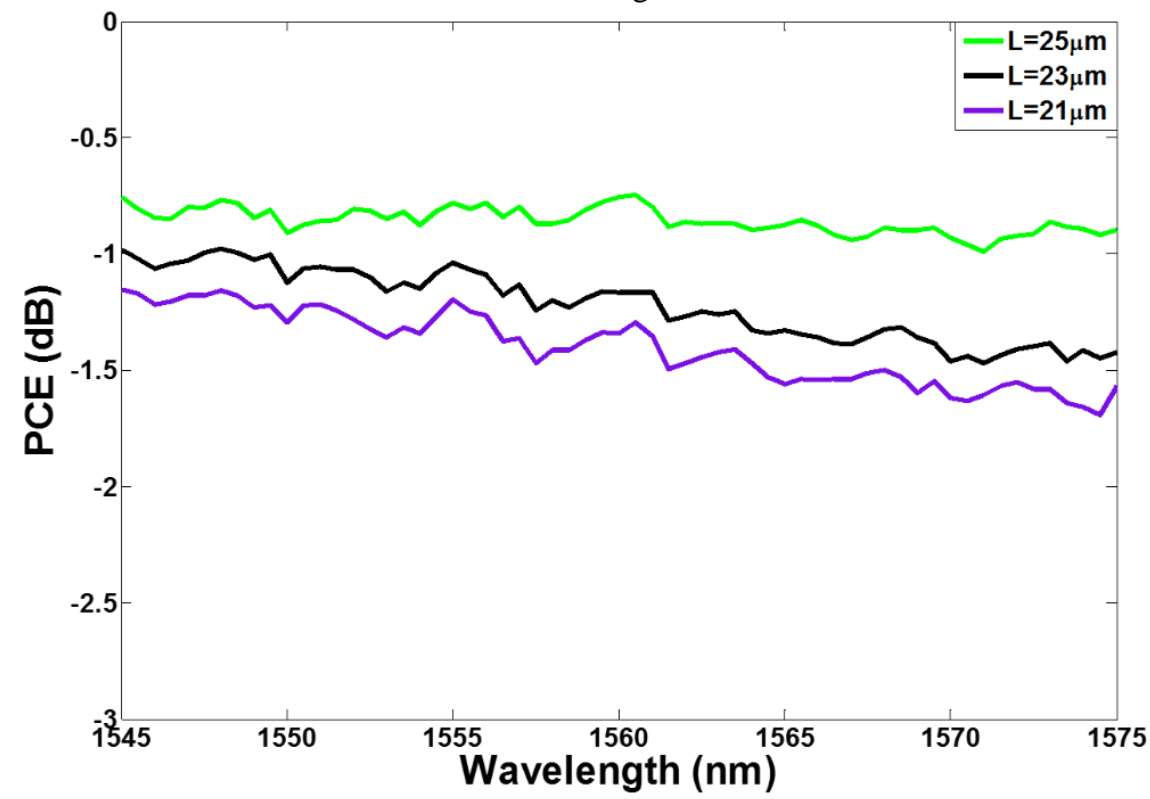

Figure 3.19: Polarization conversion efficiency as a function of wavelength for the different lengths of the polarization rotators. 
Figure 3.19 shows the PCE as a function of the wavelength, again for the three different rotator lengths. As the rotator length moves away from the optimal one, the TE-TE coupling efficiency increases, as plotted in Figure 3.18 (dashed lines), whereas the change in the TE-TM coupling (solid lines) is much lower, according with the theoretical behaviour. The measured PCE is above $-0.85 \mathrm{~dB}$ over a wavelength range of $30 \mathrm{~nm}$, and it is achieved for the polarization rotator which length is $25 \mu \mathrm{m}$. When results shown in Figure 3.14 and Figure 3.19 are compared, it can be seen that there is a very good agreement between the simulated and measured variation of the PCE with the rotator length. Small discrepancies are mainly originated due to deviations between the target and fabricated parameters of the rotator. However, it should be pointed out that the rotator parameters may change in the range of a few tens of nanometers without decreasing the PCE in more than $0.6 \mathrm{~dB}$ as shown in the robustness study carried out by simulations and depicted in Figure 3.15. Therefore, it can be stated that the robustness of the proposed polarization rotator device meets CMOS fabrication tolerances based on 193nm deep UV lithography.

\subsection{DQPSK Receiver}

\subsubsection{Introduction}

An ever growing amount of access network bandwidth is required by end users, and the deployment of passive optical networks, operating up to $10 \mathrm{Gbit} / \mathrm{s}$ has already begun to address this demand. Even higher bit rates will be required in the future, with network operators preferring solutions based on reusing the existing infrastructure and components developed for legacy links. Advanced modulation formats can enable the further scaling of the bit rates while keeping the legacy $10 \mathrm{GHz}$ devices [EFF11]. As commented previously, DPSK offers several advantages over an OOK modulation format, namely higher nonlinearity tolerance, 3-dB receiver sensitivity improvement when used with balanced detection and the possibility to operate at lower symbol rates for a given bit rate using DQPSK [GNA05b]. In the differential encoded DPSK, the information is encoded in the phase transition and can be demodulated using a passive delay-interferometer, without the need of the LO and the DSP, making differential detection advantageous for low cost links.

For multilevel receiver implementations, three different technologies are usually used, such as free-space optics, fiber optics and photonic integrated circuits (PICs). Due to the possibilities that PICs offer, as potential cost and size reduction as well as improved manufacturability and testability, this option is preferable [DOE10a] over others. Moreover, PICs can be developed either in monolithic technologies, such as InP or Si, or in planar lightwave circuit (PLC)-based technologies with hybrid integration [KRO11]. Both coherent [DOE10b] and differential [DOE08] receivers have been demonstrated in $\mathrm{InP}$, being one of the main advantage of InP receivers is that high-speed photodiodes and light sources (important for coherent receivers) are feasible in this material. However, 
silicon technology presents key advantages that make it preferable in our case: $\mathrm{Si}$ waveguides exhibit lower losses than InP, it can be used with large wafers, with a diameter of $200 \mathrm{~mm}$ or even more [DOE11] containing more devices and hence, reducing cost almost linearly to the area size. In general, silicon photonics enable re-use of existing infrastructure and research from electronic integration, together with sophisticated metrology and process control [PAR11]. Moreover, to provide high-speed and highperformance detection elements, Ge photodiodes can be heterogeneously integrated on the Si wafer, as it will be explained in this section. From the aforementioned properties, silicon can be used to implement low-cost and high-performance multilevel receivers.

As mentioned in the previous section, coherent receivers, which have been researched extensively in modern optical communication systems ([ALF09], [SLE11]), need a LO as a means to provide an absolute phase reference for the received signal.

In a MZDI-based DPSK receiver has been targeted in which the differential phase modulation is normally converted into amplitude modulation. The MZDI demodulates the differential phase between each data bit and its successor, which implements the differential decoding of DPSK modulation, by splitting up the signal in two copies, delaying one copy over a single bit period $\Delta T$ in [s] and finally recombining both arms to create optical interference, as depicted in the figure below.

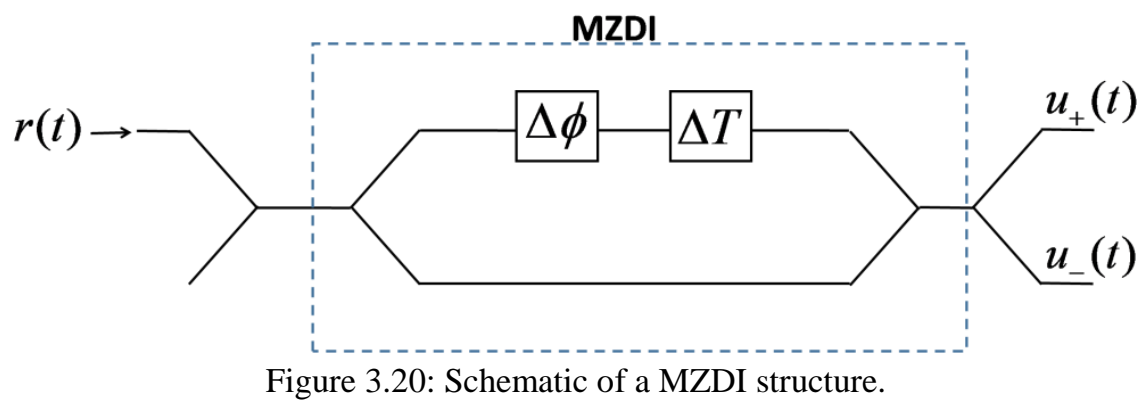

The transfer function of the MZDI is defined through:

$$
u_{ \pm}(t)=r(t) \pm \exp (j \Delta \phi) \cdot r(t-\Delta T) \quad \text { Eq. } 3.6
$$

where $u_{ \pm}(t)$ is the constructive and destructive component, respectively. $r(t)$ is the input signal and $\Delta \phi$ the phase difference between both interferometer arms. The phase difference is ideally $\Delta \phi=0 \pm \pi$. When $\Delta T$ is the delay in the interferometer arm, the constructive and destructive output ports exhibits a periodic notch response with a $3-\mathrm{dB}$ bandwidth of $1 /(2 \Delta T)$ and a free spectral range of $1 / \Delta T$. In the optical spectrum of the destructive output signal, the carrier frequency is suppressed, resulting in an AMI signal, while the signal at the constructive port is a DB one. Both the constructive and destructive output ports of a MZDI carry the full information of the DPSK signal. Therefore, detecting either only the constructive or destructive output is sufficient, and this is known as single-ended detection. But in order to obtain the 3-dB improvement in the receiver 
sensitivity over OOK modulation, both MZDI output ports must to be detected simultaneously, and this is known as balanced detection.

\subsubsection{DPSK Demodulator}

In order to compensate the propagation loss in the delay line, a MZI switch in cascade with the demodulation circuit is proposed to cope with unbalanced powers between the arms of the MZDI that would decrease the extinction ratio and hence degrade the receiver sensitivity. By using this scheme a significant increase in the ER of the MZDI can be achieved from either a passive receiver, by tuning the wavelength and so achieving zero power consumption, or a low-power receiver by using a microheater. Furthermore, the footprint is reduced by using compact spirals and so minimizes the size of the receiver to just a few times larger than a ring resonator based implementation.

Recently, an alternative implementation based on two variable optical attenuators (VOAs) coupled to the input of each waveguide of the MZDI has also been proposed to balance the output power in a MZDI based receiver [SUZ12]. However, the overall insertion loss will suffer using this implementation since extra loss is introduced by the VOAs in the circuit. A power consumption of the VOAs as high as $17 \mathrm{~mW}$ is also reported.

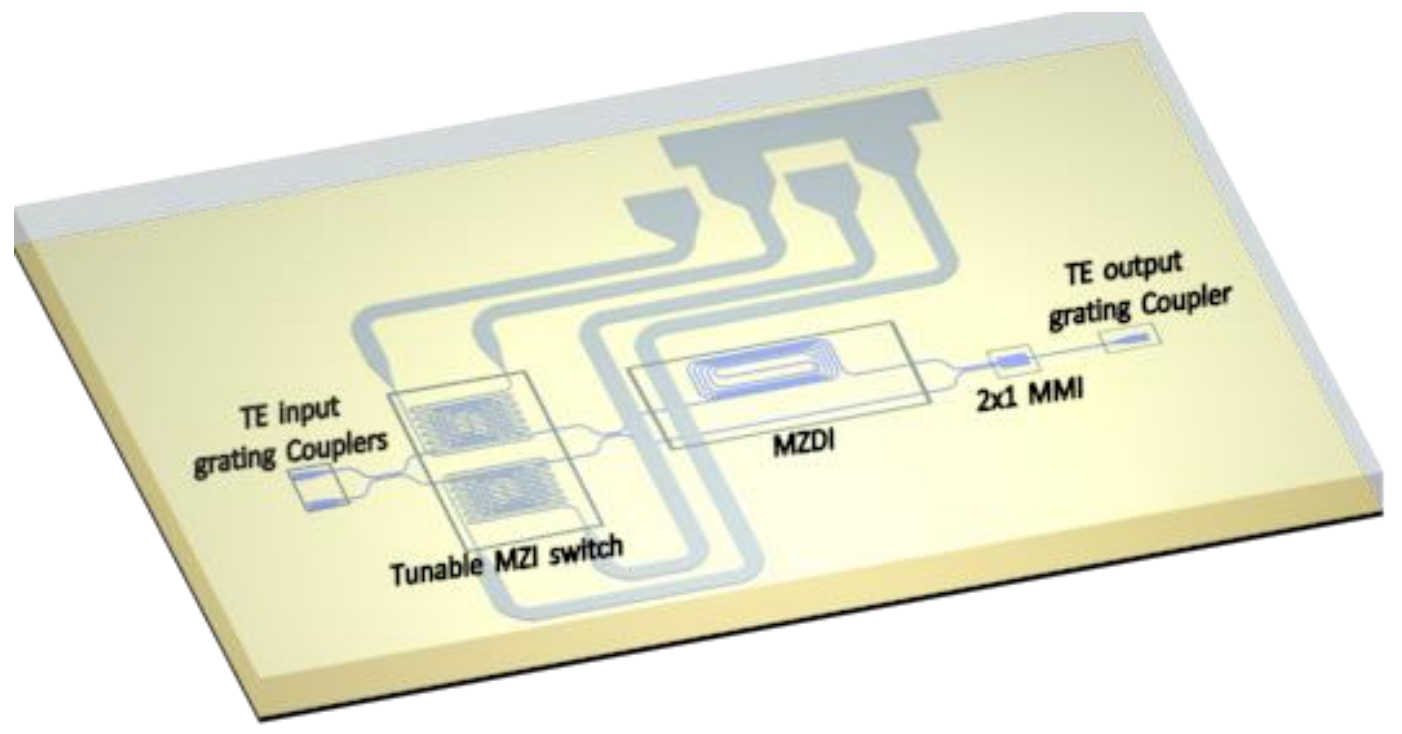

Figure 3.21: Schematic of the proposed DPSK demodulator. From left to right in the figure: Two input TE grating couplers with waveguide tapers, 2x2 unbalanced MZI switch, MZDI with a $10 \mathrm{GHz}$ DL, a 2x1 MMI, taper, and an output grating coupler.

The schematic of the proposed DPSK demodulator, as a proof of concept, is depicted in Figure 3.21. A thermo-optically tunable asymmetric MZI switch is placed in series with the $10 \mathrm{Gbit} / \mathrm{s}$ MZDI DPSK demodulator. The $10 \mathrm{Gbit} / \mathrm{s} 1$-bit DL is of the order of about $1 \mathrm{~cm}$. There are two identical inputs to the circuit and at the output of the MZDI a 2x1 MMI is used. To take advantage of balanced detection this MMI should be replaced by a $2 \times 2$ one. Anyhow, in our test-setup we are not using balanced detection, but single detection, and hence the second output is obsolete. SOI strip waveguides with a size of 
$220 \times 450 \mathrm{~nm}$ and surrounded by a $\mathrm{SiO}_{2}$ cladding are considered. Hence, the proposed device is optimized for TE polarization and TE gratings are used to inject/extract the light. The asymmetric MZI switch has a length of approximately $500 \mu \mathrm{m}$ in the shorter arm and a path difference of $50 \mu \mathrm{m}$ between both arms. The microheaters have approximately the same length and width than the optical waveguides in the MZI arms and follow the same spiral shape shown in Figure 3.21.

Figure 3.22 shows the simulated spectral response of the DPSK demodulator using the transfer matrix method [POO04]. In the simulation the 1-bit DL was set to fit a 10G symbol rate being waveguide propagation loss of $6 \mathrm{~dB} / \mathrm{cm}$. Propagation loss is higher than state-of-the art values, however it better fits the actual fabricated waveguide used here in the characterized device. DPSK demodulation can be carried out at the minimum in the transmission spectrum, given rise to an AMI modulation format, or at the maximum transmittance, given rise to a DB modulation format. To prove the increase in performance we will use the AMI modulation format at a minimum transmission, however DB modulation could be used in an identical manner. In both cases, the ER between minimum and maximum transmission should be maximized to enhance the sensitivity of the receiver. To achieve this it is important that the output power in the arms of the MZDI, or in other words the amplitudes of the two adjacent bits, are identical so they completely cancel each other out maximizing the ER.

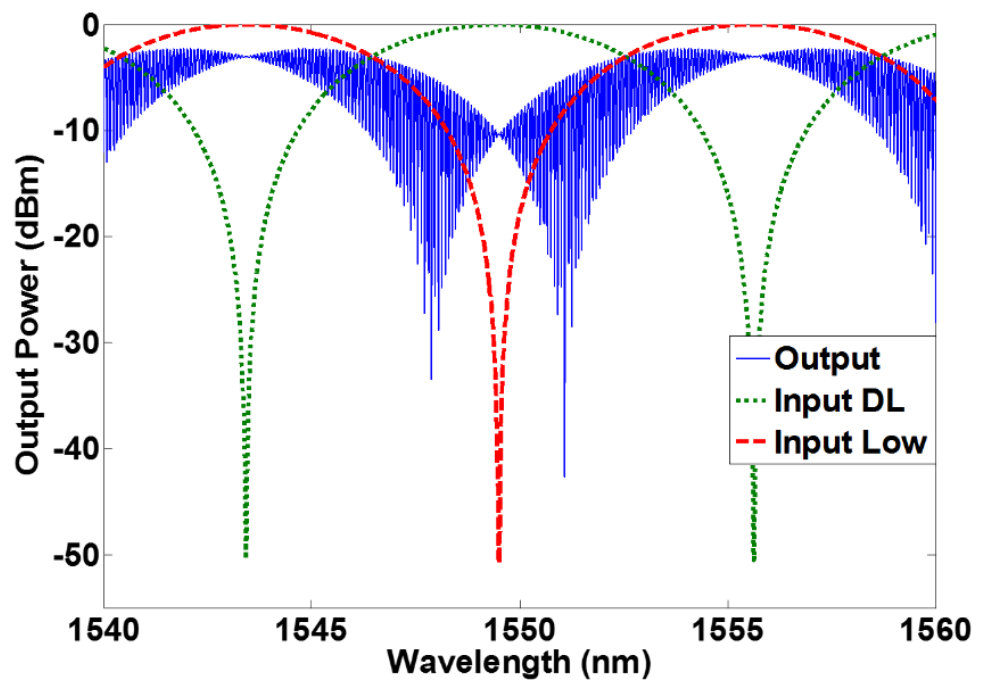

Figure 3.22: Simulated spectral response at the MZDI inputs (green-dotted, red-dashed) and at the demodulator output (blue-solid), taking into account $6 \mathrm{~dB} / \mathrm{cm}$ propagation losses.

To reflect the behaviour of the MZI switch for increasing the ER, Figure 3.22 includes the input power to each arm of the MZDI; the green dotted curve is the power at the input of the DL arm of the MZDI and the red dashed curve for the opposite arm. The two curves cross at $3 \mathrm{~dB}$, which corresponds to the standard 50:50 splitting ratio. It is clear from the figure that the ER at 50:50 is not the optimized value, but closer to 65:35, in order to compensate for the propagation losses in the DL. The simulation predicts an increase in ER from around $13 \mathrm{~dB}$ at 50:50 to more than $30 \mathrm{~dB}$ at maximized performance. It should 
be pointed out that supposing state-of-the art propagation losses [DUM04], the theoretically maximum ER will still be limited to $16 \mathrm{~dB}$ for a standard 50:50 splitting ratio and therefore the proposed technique will also be required to enhance the receiver sensitivity. The impact will be lower for DPSK receivers designed for a higher bit rate because the DL will have a shorter length and thus losses will be lower. For instance, for $40 \mathrm{Gbit} / \mathrm{s}$ operation, the theoretically maximum ER will be around $28 \mathrm{~dB}$ supposing stateof-the art propagation losses. However, even in this case, a fine tuning of the splitting ratio would be useful for compensating potential undesired variations in the fabrication process that could degrade the ER.

On the other hand, the $50 \mu \mathrm{m}$ path difference in the MZI switch results in a free spectral range (FSR) 160 times larger than the FSR of the MZDI, which means that between two minima of the MZI switch, there will be around 160 minima of the MZDI. Two of these set of minima would show the maximized ER, as shown in Figure 3.22, and thus increased sensitivity of the receiver without requiring any active tuning on the chip. For enhanced performance it is important to use a good ratio between these two FSRs. A too high ratio, i.e. a shorter path difference in the MZI switch, would not achieve the resolution between local minima needed to achieve a global optimum minimum, and a too low ratio, i.e. a longer path difference in the MZI switch, could possibly result in no optimized resonance within the operational bandwidth of the receiver.

The DPSK receiver was fabricated at the NTC's facilities, on SOI wafer with silicon core thickness of $220 \mathrm{~nm}$ and buried oxide (BOX) of $2 \mu \mathrm{m}$, and covered by a $1 \mu \mathrm{m}$-thick silica overcladding. The fabrication process was carried out by using electron beam lithography and dry etching by using inductively coupled plasma (ICP) system. Plasma enhanced chemical vapour deposition (PECVD) was also used, to grow the overcladding silica layer. Figure 3.23 shows images of the fabricated MZI switch and delay-line structure.

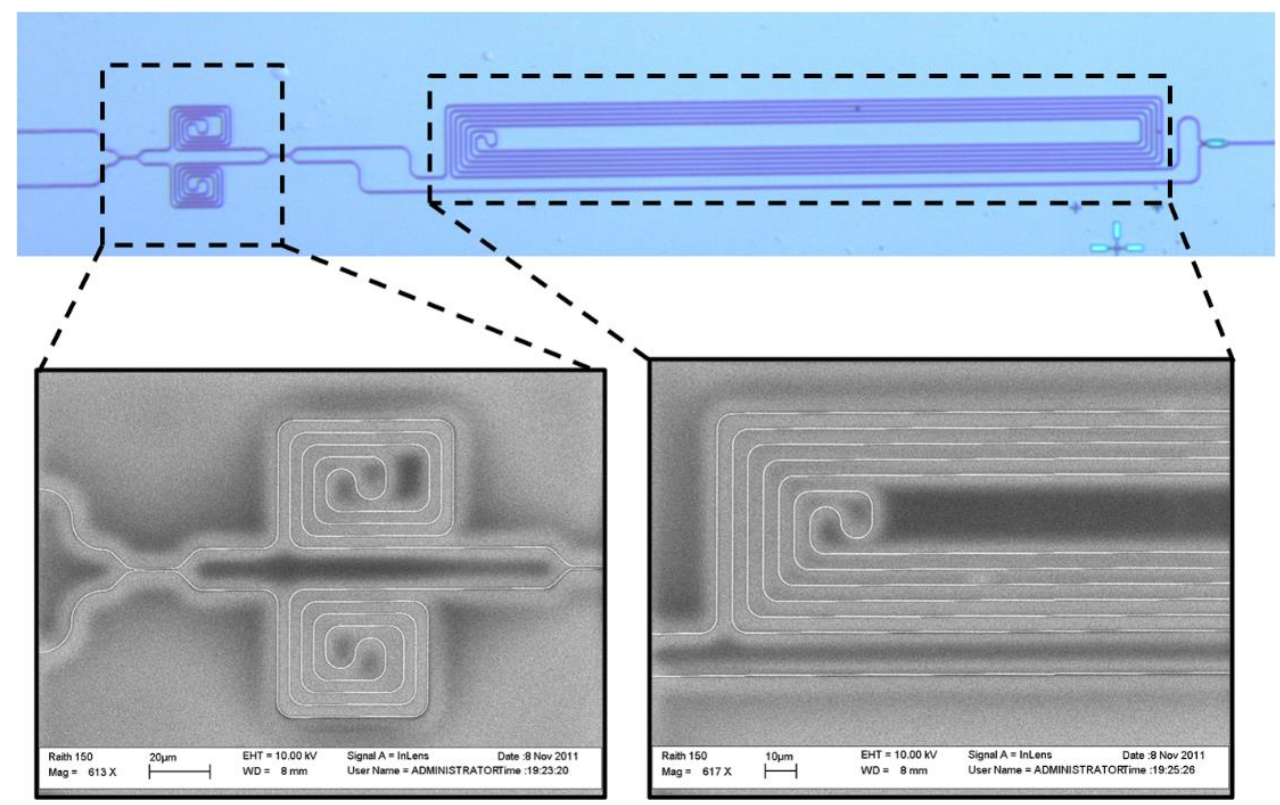

Figure 3.23: Optical photo of the MZI-MZDI structure. Below the left and right inset show a SEM image of the MZI with and MZDI spirals, respectively. 
Continuous-wave light was generated, using the setup shown in Figure 3.17, by an external cavity laser and was first swept over the full operational wavelength bandwidth, from $1545 \mathrm{~nm}$ to $1565 \mathrm{~nm}$. Figure 3.24(a) shows the obtained experimental spectrum, including zoom on the optimized performances in Figure 3.24(b) around $1555.5 \mathrm{~nm}$. The experimental spectrum is in good agreement with the simulation result in Figure 3.22. Maximized performance is achieved at approximately 65:35 splitting ratio, as predicted by simulation, resulting in $28 \mathrm{~dB}$ ER marked as R2 in Figure 3.24(b). The 50:50 splitting ratio is estimated to about $11 \mathrm{~dB}$. The expected $16 \mathrm{~dB}$ resonance, if propagation losses would be reduced to state-of-the art values, is marked with $\mathrm{R} 1$. To estimate the increase in the receiver sensitivity, the performance at $\mathrm{R} 1$ will be compared with $\mathrm{R} 2$.
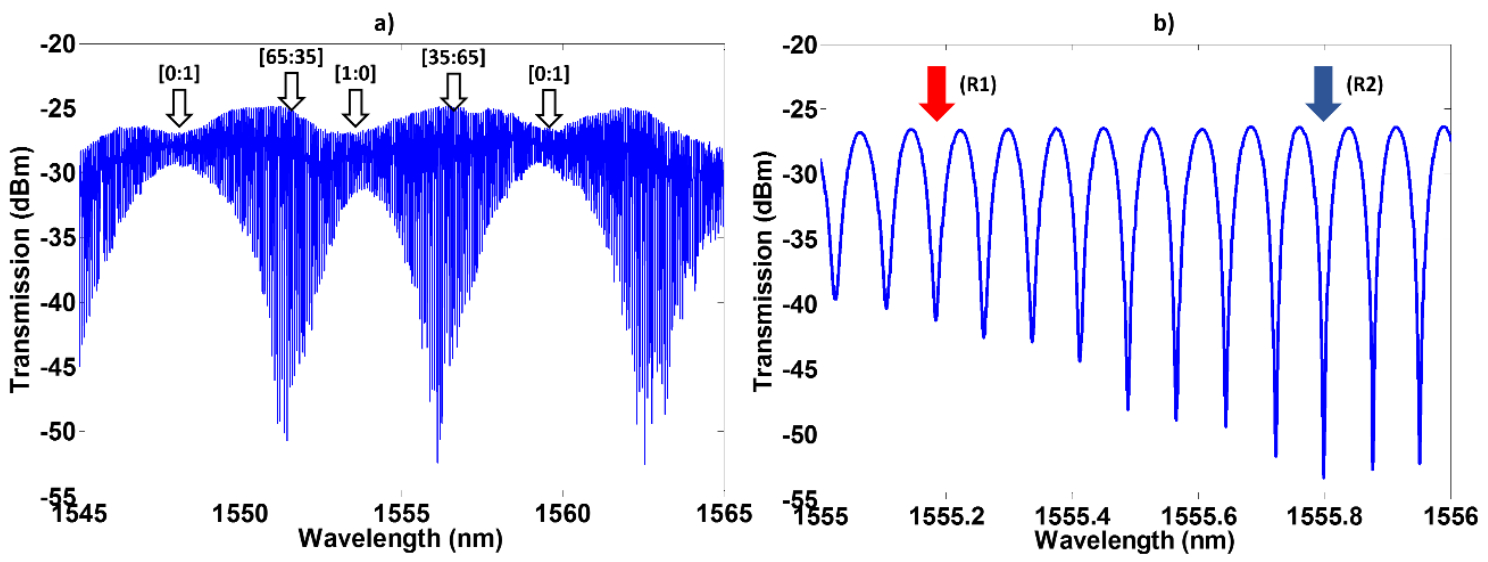

Figure 3.24: Measured spectral response at the DPSK demodulator. (a) Transmission spectrum of the full experimental wavelength band. The arrows mark the different states of the MZI switch at the input of the MZDI. (b) A zoom with 1pm resolution on the wavelength range where resonances have higher ER. Two resonances are marked in the figure: R1 for $16 \mathrm{~dB}$ ER and R2 with optimized $28 \mathrm{~dB}$ ER.

As already stated, instead of varying the wavelength, by including microheaters on the MZI switch, the ER of any resonance could be tuned by means of the thermo-optic effect. Figure 3.25 shows the ER variation for the resonance at 50:50 splitting ratio with different electrical power applied to the micro-heater on the MZI switch. Inset shows an optical photo of the metal micro heaters on-top of the MZI switch. Heaters and contact pads were sequentially patterned with lithography (PMMA resist), evaporation, and lift-off processes and consist of $115 \mathrm{~nm}$ thick Titanium. The heater follows the same pattern as the waveguides with a width of $500 \mathrm{~nm}$.

The result shown in Figure 3.25 demonstrates how the ER is increased from around 11 $\mathrm{dB}$ at $0 \mathrm{~mW}$ to above $25 \mathrm{~dB}$ ER at $3 \mathrm{~mW}$ tuning power. The efficiency could further be increased by isolating the heater and etching trenches around the structure to avoid heat dissipation [WAT08]. If using both arms of the MZI switch, the phase can be both increased and decreased so to maximize any resonance the tuning power is equal or less than a phase shift of $\pi$, here corresponding to less than $5 \mathrm{~mW}$. 


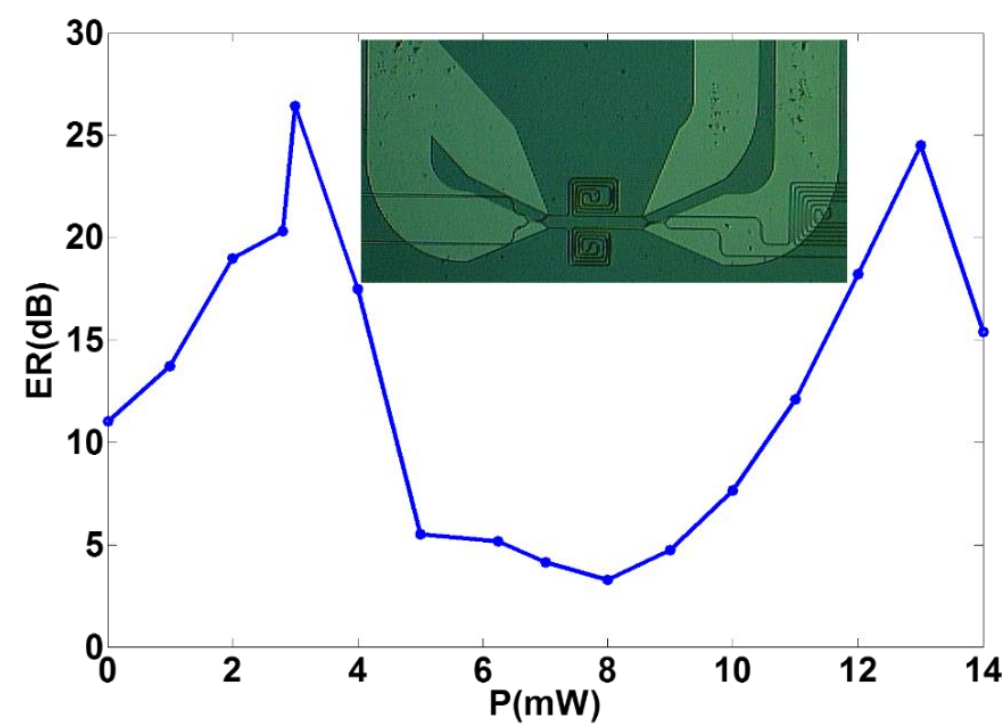

Figure 3.25: ER variation for different powers applied to the micro-heater on the MZI switch. The inset shows an optical image of the microheaters on top of the MZI switch.

For system measurements, the two resonances marked in Figure 3.24(b) were selected; R2 with maximized performance of $28 \mathrm{~dB}$ ER of the MZDI at $1555.80 \mathrm{~nm}$, and R1 with non-optimized performance of $16 \mathrm{~dB}$ at $1555.19 \mathrm{~nm}$.

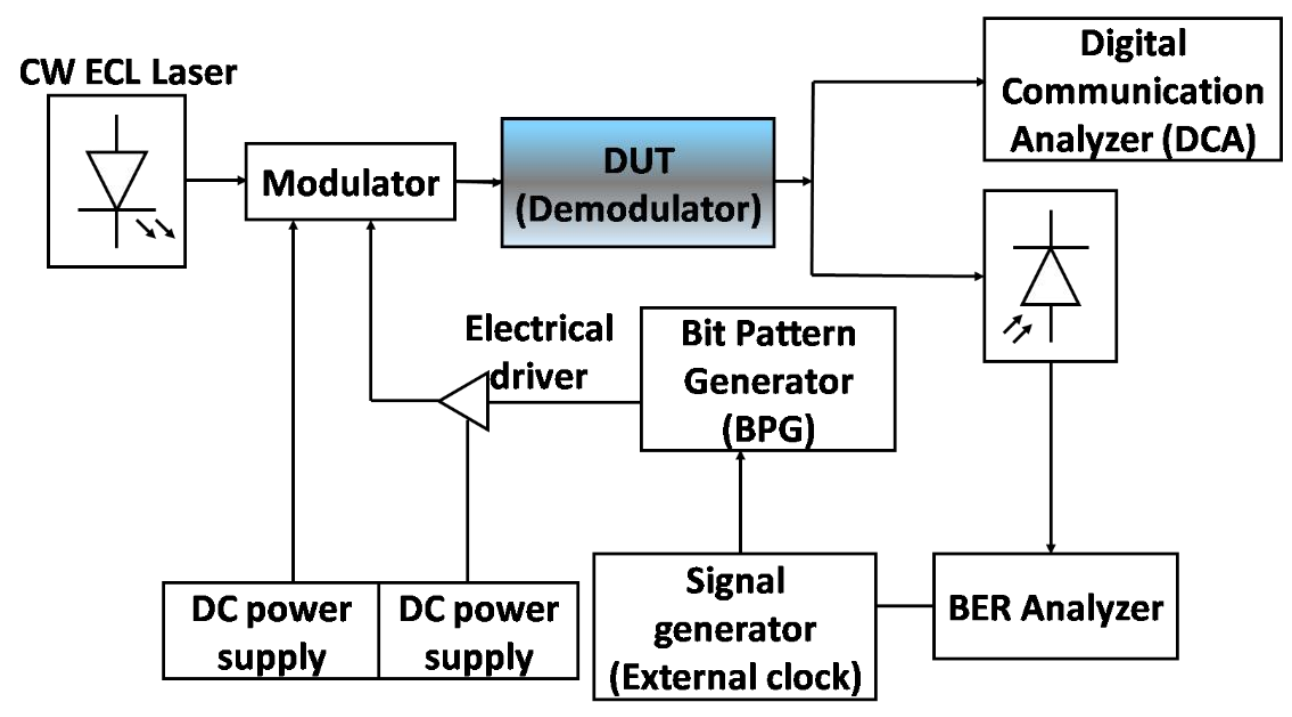

Figure 3.26: Experimental set-up used for the BER measurements.

Figure 3.26 shows the experimental DPSK demodulation setup. To test the demodulation circuit, an optical data stream was generated using an X-cut Lithium Niobate modulator biased at minimum with $2 \mathrm{~V}_{\pi}$ driving voltage. The bits were generated from a pseudorandom binary sequence pattern generator (PRBS) with a pattern length of $2^{31}-1$ at $10 \mathrm{Gbit} / \mathrm{s}$ bit-rate, delivered by a bit pattern generator (SHF BPG 44E) connected to an external clock. At the output of the chip, the demodulated signal was photo-detected by a Digital Communication Analyzer (Infinium DCA-J 86100C), and simultaneously examined on a bit error rate analyzer (SHF EA 44). The total measured insertion-loss of 
the chip is $21 \mathrm{~dB} ; 7 \mathrm{~dB}$ losses at each grating coupler and $7 \mathrm{~dB}$ propagation losses including about $2 \mathrm{~dB}$ losses from the MMIs.

a)

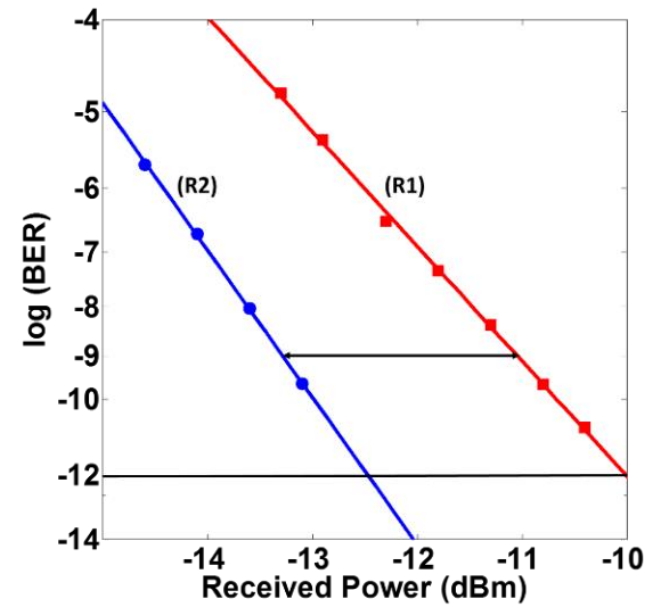

b)

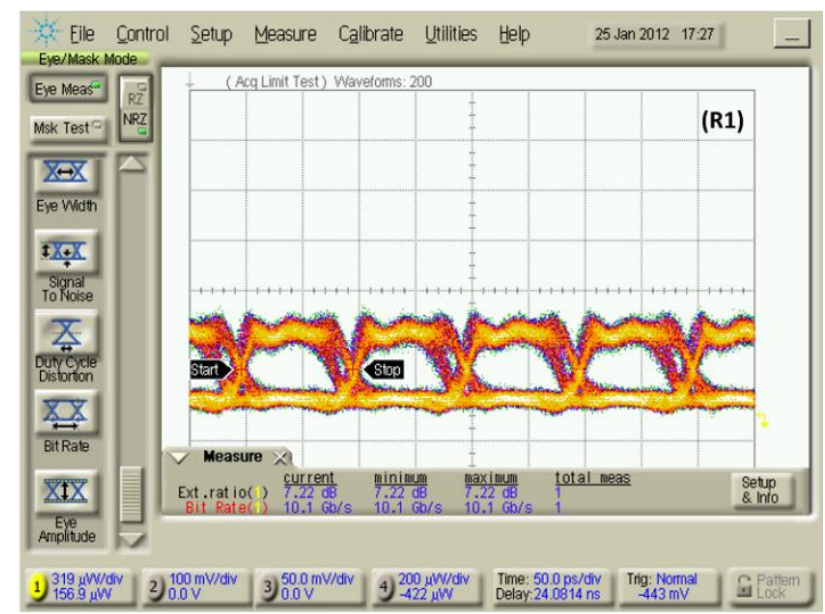

c)

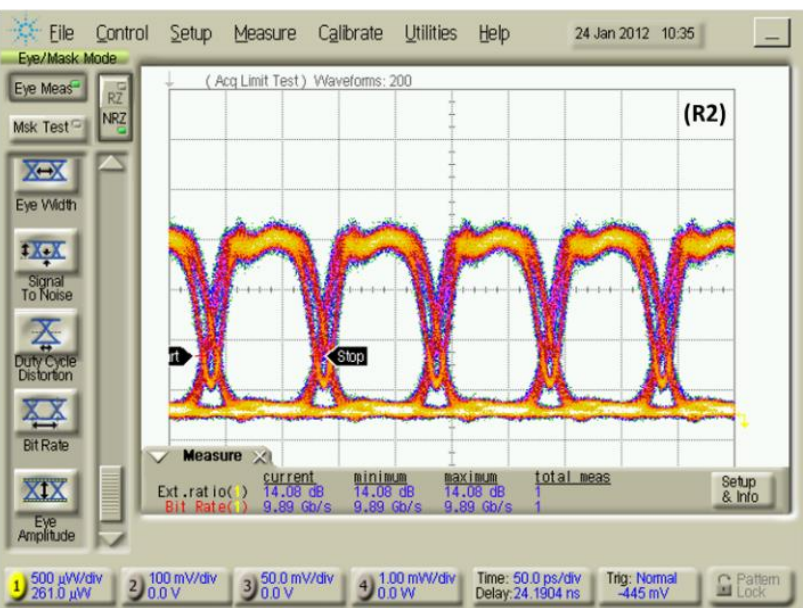

Figure 3.27: System measurements at 10 Gbps of the DPSK demodulator. (a) BER measurements for the two studied resonances, R1 (red) and R2 (blue), marked in Figure 3.24. (b-c) The corresponding eye diagrams for $\mathrm{R} 1$ and $\mathrm{R} 2$ respectively. 
Figures 3.27(b) and 3.27(c) show the eye-diagrams of the two demodulated signals. It is clear that the eye is more open for the optimized resonance R2 than for R1, being the measured ER significantly improved from $7 \mathrm{~dB}$ to $14 \mathrm{~dB}$. Finally, in order to estimate the increase in sensitivity of the receiver, the BER was also measured for the two resonances. Error-free operation was achieved, and the measured bit-error-rate curves are plotted in Figure 3.27(a). The two curves are almost parallel and an increase in sensitivity of about $2.3 \mathrm{~dB}$ is measured at a BER of $10^{-9}$.

These obtained results show a proof of concept how to maximize the sensitivity of low bitrates low-cost differential MZDI receivers with minimum power consumption, with an energy efficient approach and minimizing the foot-print of the receiver, in order to overcome the limitation of the ER of the unbalanced MZDI produced by DL propagation losses by using an unbalanced thermo-optically tunable MZI switch in series with the MZDI.

\subsubsection{DQPSK Demodulator with Balanced Detection}

Once the DPSK demodulator has been demonstrated, the next step is to go further by demodulating a DQPSK signal and its detection by using two integrated and balanced photodetectors. The incoherent or differential detection scheme can recover twodimensional modulation formats with no absolute phase reference, since it is provided by the received signal itself, delayed by one symbol period, by beating it with the incoming signal. This means that the information that is available in the receiver is only the phase difference between two consecutive symbols, implying that the transmitted symbol itself will not be recovered in the receiver; therefore the transmitted data must be encoded in the phase difference between the symbols. The two most widely used implementations of differential receivers are based on a $90^{\circ}$ hybrid with a $3 \mathrm{~dB}$ splitter and a DL to create the delayed signal, or on two asymmetric MZDIs. In order to achieve a minimum footprint of the device, the selected option is to use the $90^{\circ}$ hybrid for the DQPSK receiver implementation, as depicted in Figure 3.28.

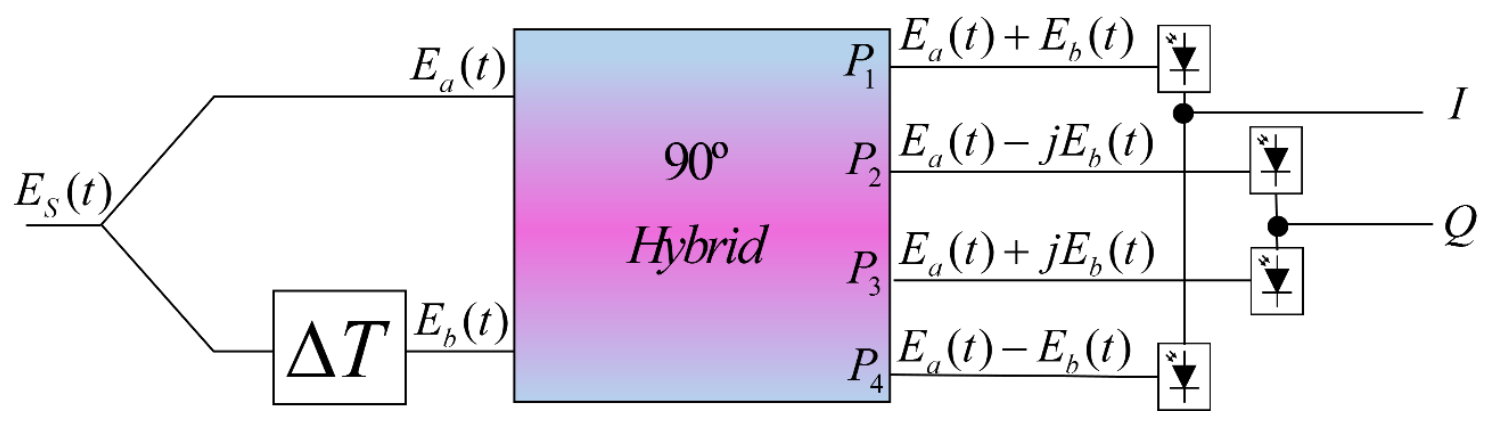

Figure 3.28: Schematic of a differential receiver with balanced detection

In general, the outputs of the hybrid as a function of the input signals $E_{a}(t)$ and $E_{b}(t)$ can be expressed as described in Eq. 3.7: 


$$
\begin{aligned}
& E_{1}(t)=E_{a}(t)+E_{b}(t) \\
& E_{2}(t)=E_{a}(t)-j E_{b}(t) \\
& E_{3}(t)=E_{a}(t)+j E_{b}(t) \\
& E_{4}(t)=E_{a}(t)-E_{b}(t)
\end{aligned}
$$

being $E_{a}(t)=\left|E_{a}(t)\right| e^{j \theta_{a}(t)}$ and $E_{b}(t)=\left|E_{b}(t)\right| e^{j \theta_{b}(t)}$. When balanced detection is implemented, the currents from the second and the fourth photodiodes are subtracted from the currents of the first and the third, respectively. This can be done on the photonic chip itself, e.g. by connecting every two photodiodes in series, so one balanced pair detects the imaginary part of the signal (Q) while the other detects the real part (I). Furthermore, since the phase difference between pairs of ports $(1-4,2-3)$ is always $180^{\circ}$, balanced detection is used to eliminate the DC component. The output powers will be:

$$
\begin{gathered}
P_{1}(t) \propto\left|E_{a}(t)\right|^{2}+\left|E_{b}(t)\right|^{2}+2\left|E_{a}(t)\right| \cdot\left|E_{b}(t)\right| \cdot \cos \left[\theta_{a}(t)-\theta_{b}(t)\right] \\
P_{2}(t) \propto\left|E_{a}(t)\right|^{2}+\left|E_{b}(t)\right|^{2}-2\left|E_{a}(t)\right| \cdot\left|E_{b}(t)\right| \cdot \sin \left[\theta_{a}(t)-\theta_{b}(t)\right] \\
P_{3}(t) \propto\left|E_{a}(t)\right|^{2}+\left|E_{b}(t)\right|^{2}+2\left|E_{a}(t)\right| \cdot\left|E_{b}(t)\right| \cdot \sin \left[\theta_{a}(t)-\theta_{b}(t)\right] \\
P_{4}(t) \propto\left|E_{a}(t)\right|^{2}+\left|E_{b}(t)\right|^{2}-2\left|E_{a}(t)\right| \cdot\left|E_{b}(t)\right| \cdot \cos \left[\theta_{a}(t)-\theta_{b}(t)\right] \\
i_{I, B D}(t) \propto P_{1}(t)-P_{4}(t), \quad i_{Q, B D}(t) \propto P_{3}(t)-P_{2}(t)
\end{gathered}
$$

Regarding the relations shown in Figure 3.28, $E_{a}(t)=E_{S}(t)=\left|E_{S}(t)\right| e^{j \theta_{S}(t)}$ and $E_{b}(t)=E_{S}(t-\Delta T)=\left|E_{S}(t-\Delta T)\right| e^{j \theta_{S}(t-\Delta T)}$, and if we take $\Delta \theta(t)=\theta_{S}(t)-\theta_{S}(t-\Delta T)$ we obtain that the resulting currents will be:

$$
\begin{aligned}
& i_{I, B D}(t) \propto \cos [\Delta \theta(t)] \\
& i_{Q, B D}(t) \propto \sin [\Delta \theta(t)]
\end{aligned}
$$

From Eq. 3.9, it can be seen that there is no frequency offset. That means that there is no need for frequency and carrier phase estimation in the receiver. Essentially, the phase difference between the symbols is translated to amplitude information, which can be directly used for data decoding. This simplicity is the main reason that differential modulation formats were the first to be exploited in the race towards increased spectral efficiency [GRI02a].

A complete integrated differential DQPSK receiver on SOI, including Ge-balanced photodetectors (Ge-BPD) [AAM13b] was developed. Integrated differential receivers based on $90^{\circ}$ hybrids in Si have been presented in the literature [DOE10c, SUZ12]. The advantage of this design is that it uses zero-biased photodiodes, so no decoupling capacitor is required, enabling compact devices. Moreover, the MMI-based $90^{\circ}$ hybrid reduces the footprint of the device and ensures very good phase properties [HAL11]. 
The schematic of the proposed receiver is depicted in Figure 3.29. A thermo-optically tunable MZI power splitter is placed in series with a MZDI and a $2 \times 4 \mathrm{MMI}$ acting as $90^{\circ}$ hybrid. At the output, the in-phase and quadrature signals are detected using two BPDs in pinpin configuration. In the following paragraphs the implementation and optimization of each of these elements is described.

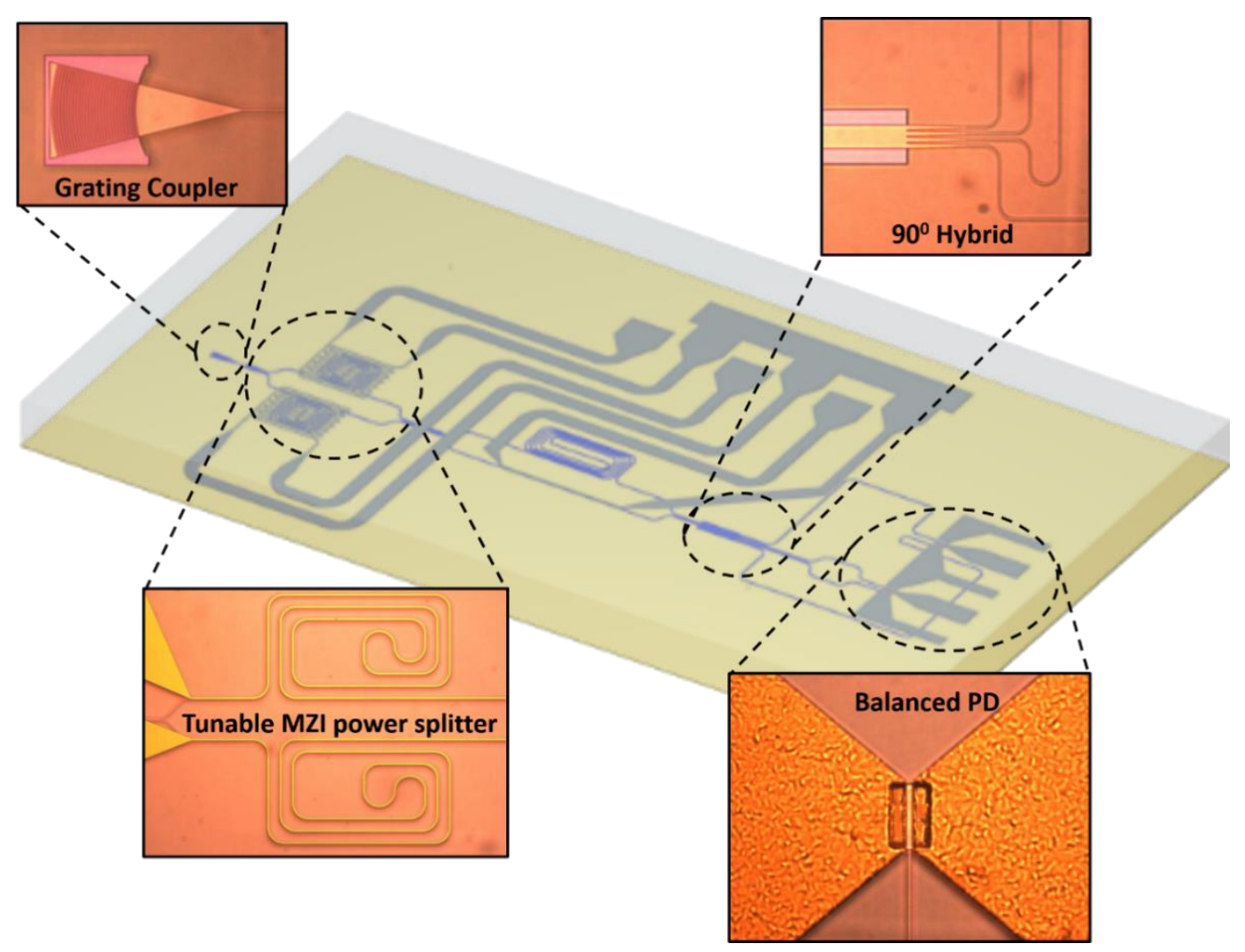

Figure 3.29: DQPSK differential receiver. (a) GDS design and (b) optical photograph of fabricated device. The receiver is assembled using 5 components, from left to right in the figure: curved grating coupler, MZI tunable power splitter, DLI, $90^{\circ}$ hybrid and two balanced PDs.

The proposed device was fabricated on top of a 200-mm SOI wafer with silicon core thickness of $220 \mathrm{~nm}$ and buried oxide of $2 \mu \mathrm{m}$. To couple light from the input fiber to the receiver, and in order to minimize the size of the chip, curved gratings [VAN07] were used, showing a coupling loss of about $6 \mathrm{~dB}$. The next elements are a tunable MZI power splitter in series with a MZDI, as described in the previous section. Compact spirals were used in order to minimize the size of the structure. Besides the $10 \mathrm{Gbit} / \mathrm{s}$ DQPSK receiver demonstration, which is the goal of this section, $20 \mathrm{Gbit} / \mathrm{s}$ and $40 \mathrm{Gbit} / \mathrm{s}$ DQPSK receivers were also considered in order to test higher speed capabilities.

A waveguide length of $14 \mathrm{~mm}$ and $7 \mathrm{~mm}$ was chosen for $10 \mathrm{Gbit} / \mathrm{s}$ and $20 \mathrm{Gbit} / \mathrm{s}$ operation respectively, introducing $\sim 2.3 \mathrm{~dB}$ and $\sim 1.16 \mathrm{~dB}$ propagation losses, respectively, taking into account that the index group value is 4.3 , the measured propagation losses are about $1.66 \mathrm{~dB} / \mathrm{cm}$, and that the delays are 200ps and 100ps, respectively. The MZDI outputs were coupled to the $2 \times 4 \mathrm{MMI}$ inputs. This MMI, with an insertion loss of about $6.5 \mathrm{~dB}$, acted as a $90^{\circ}$ hybrid [HAL11] instead of the standard implementation of a DQPSK receiver with two parallel MZDI coupled to a $180^{\circ}$ hybrid, in accordance with the 
intention of minimizing the chip area, as well as the number of active controls needed for tuning. In order to have a $3 \mathrm{~dB}$ increase in the sensitivity of the receiver and minimize power consumption, zero bias balanced detection was used based on a Ge photodetector pair (Ge-BPD) in lateral pinpin configuration. Hence, the photodetected signal is directly extracted from one single central pad avoiding additional wire bonding or metal connections. The $10 \mu \mathrm{m}$-length Ge-BPD was selectively grown at the end of silicon waveguides, and it is comparable to the ones used in [VIV12].

The fabrication process, performed at LETI's facilities, carried out on SOITEC optical SOI with $220 \mathrm{~nm}$ Si on $2 \mu \mathrm{m} \mathrm{BOX}$, starts with the deposition of $100 \mathrm{~nm}$ high temperature oxide on top of the silicon layer. The gratings and the waveguide arms are first patterned, followed by RIE silica etching with $\mathrm{C}_{4} \mathrm{~F}_{8}$, which defines a hardmask. The silicon is then partially etched $(65 \mathrm{~nm})$ with $\mathrm{HBr}$ and controlled by ellipsometry in order to define precisely the grating teeth depth. In the second lithography step, the gratings are protected by the resist and the remaining hardmask serves for the waveguides in a self-alignment process. Then a full silicon etch down to the box completes the waveguide fabrication. We then defined cavities for the selective epitaxial growth of Germanium. This is achieved by deposition of a silica layer which is etched at the end of waveguides. In order to achieve direct coupling, the silicon part of the cavities is etched down to $50 \mathrm{~nm}$ on top of the BOX. Germanium was then selectivity grown in the cavities and CMP used to adjust the thickness around $300 \mathrm{~nm}$. The doped regions ( $\mathrm{N}$ and $\mathrm{P}$ ) of the lateral $\mathrm{Ge}$ photodetector are defined sequentially by ion implantation of phosphorus and boron. A $400 \mathrm{~nm}$ thick $\mathrm{SiO}_{2}$ was deposited and a deposition and etching of $100 \mathrm{~nm}$ of Ti/TiN defined the heaters. Then after deposition of $500 \mathrm{~nm}$ of $\mathrm{SiO}_{2}$ and two-step openings, the electrodes were defined by Ti/TiN/AlCu metal stack deposition and $\mathrm{Cl} 2$ etching.

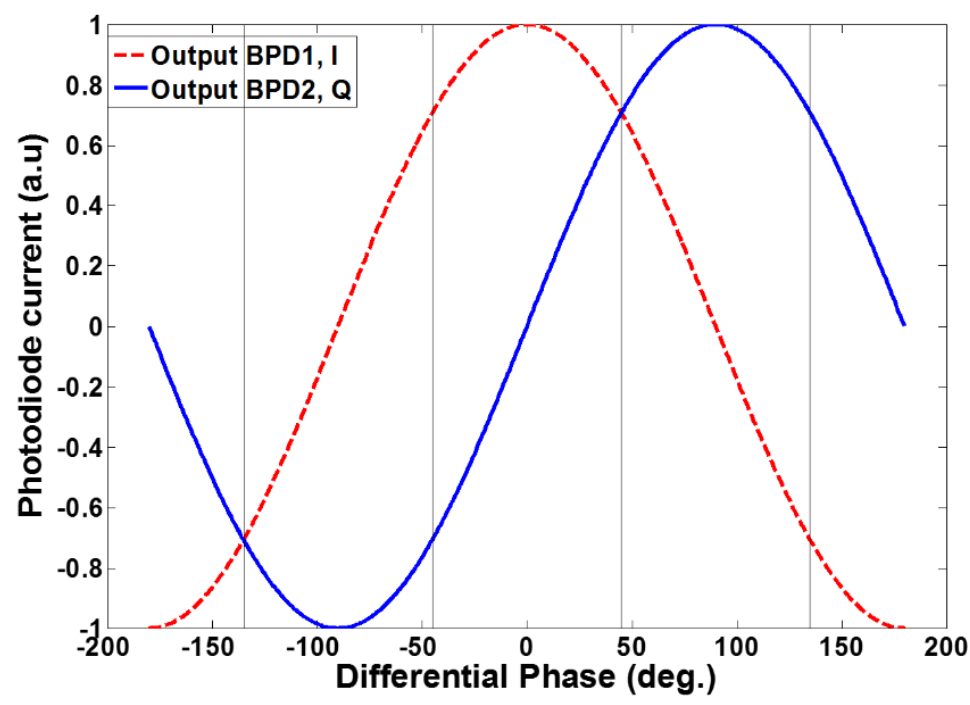

Figure 3.30: The simulated output of the receiver. The two curves represent how the in-phase and quadrature detected current from the two diodes varies with the differential phase. The four different twobits states are marked as dotted lines at $-135,-45,45$, and +135 degrees, corresponding to [-1 -1], [+1 -1], $[+1+1]$, and $[-1+1]$, respectively 
Before performing the experimental measurements, we simulated the spectral response of the MZDI and the two balanced photodiodes using the transfer matrix method [POO04], as depicted in Figure 3.30. On the $\mathrm{x}$-axis is the differential phase with respect to the two adjacent bits. The two curves are shifted exactly 90 degrees with respect to each other in order to obtain the four different states on the DQPSK modulation format. The states are marked in the figure as dotted vertical lines at the four different orthogonal phases at, $-135,-45,+45$, and +135 degrees.

The responsivity is measured at $1 \mathrm{~A} / \mathrm{W}$ at $1550 \mathrm{~nm}$ using 0 to $-2 \mathrm{~V}$ bias. Figure 3.31(a) shows the measured Dark and Light current of a single PD. For 0V bias, which will be the operation bias in our system, the Dark current is below $0.1 \mathrm{nA}$.
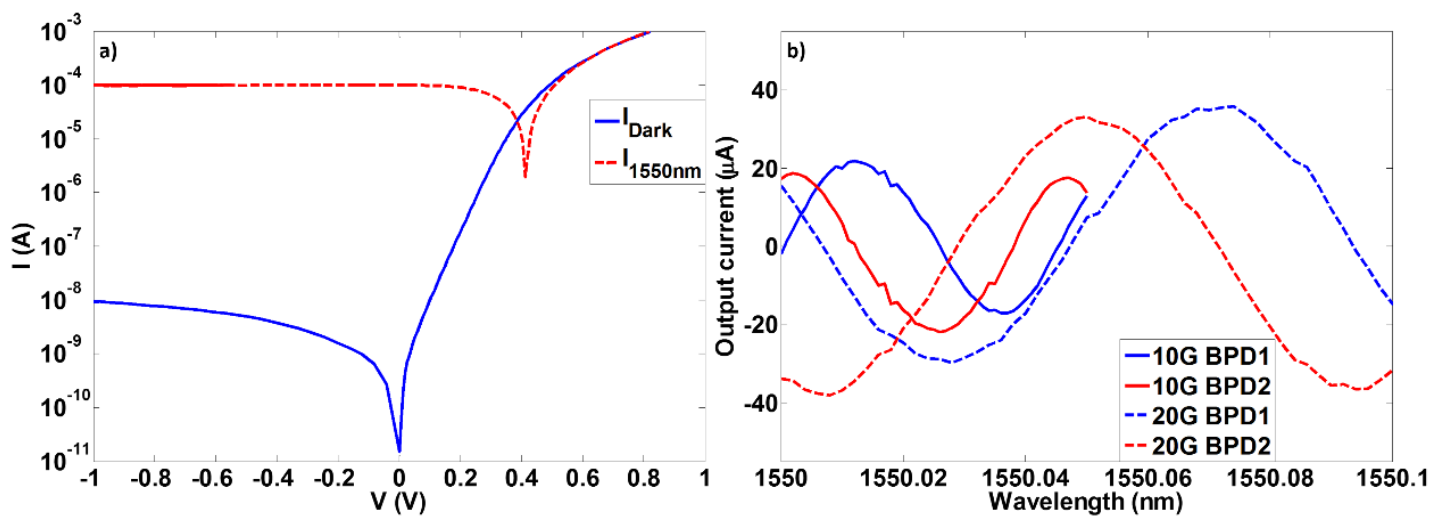

Figure 3.31: (a) The Dark (solid blue) and Light (dashed red) current measured for the fabricated photo diodes. (b) Output current as a function of wavelength for the $10 \mathrm{Gbit} / \mathrm{s}$ (solid lines) and $20 \mathrm{Gbit} / \mathrm{s}$ (dashed lines) bit rate receiver. The blue and the red lines correspond to the in-phase (I) and quadrature (Q) outputs, respectively.

For an input power of $-3 \mathrm{dBm}$, the transmission spectra of the two BPDs were measured, for both receivers. As it can be seen in Figure 3.31(b), the spectra have excellent agreement with the expected behaviour for a DQPSK receiver shown in Figure 3.30. For both receivers, the two outputs are perfectly 90 degree phase shifted with respect to each other confirming the correct behaviour of the $90^{\circ}$ hybrid. The performance, in terms of responsivity, of our Ge-BPDs is quite better than those presented in [KRO11, FAR12, $\mathrm{XU12}$ ]. On the other hand, the total optical excess loss of our proposed device is around $15.8 \mathrm{~dB}$ and $14.65 \mathrm{~dB}$ for the $10 \mathrm{Gbit} / \mathrm{s}$ and $20 \mathrm{Gbit} / \mathrm{s}$ respectively, values of the same order as those presented in [DOE12].

The setup used to test the high speed behaviour of the receiver is shown in Figure 3.32. A DQPSK signal was generated using a commercial single-drive Lithium Niobate nested MZ modulator, biased at minimum transmission and driven by the outputs of the pulse pattern generator (PPG), appropriately decorrelated, aligned and amplified at $2 \mathrm{~V}_{\pi}$. The bits were generated from a PRBS with a pattern length of $2^{13}-1$, which was the longest sequence that could be used to create a custom pattern sequence. This custom sequence was used to allow for the insertion of a preamble that facilitated the discovery of the start of the sequence of the detected data. The generated DQPSK signal passes through a VOA, which is the reference point for the received power, and is then amplified by an erbium 
doped fiber amplifier (EDFA). At the output of the chip, the electrical signals were extracted directly from the photodiodes using RF probes in a GSGSG configuration, and were transferred to two amplifiers, and then were sampled by the digital phosphor oscilloscope (DPO) for further processing off-line. Two post-processing algorithms were introduced, which are the I/Q imbalance compensation and the symbol phase estimation for rotation compensation [SOT13].

a)

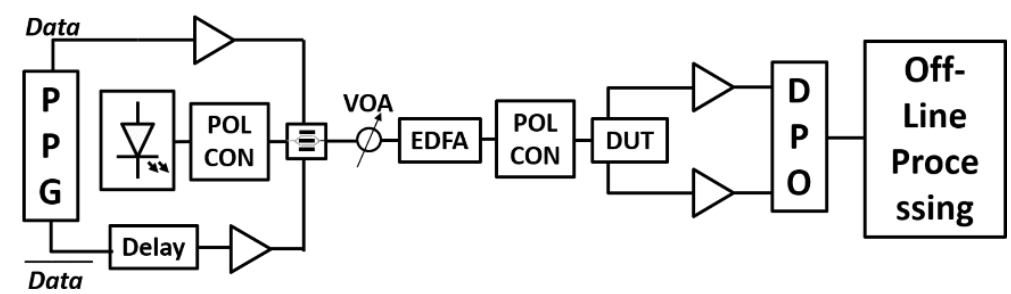

b)

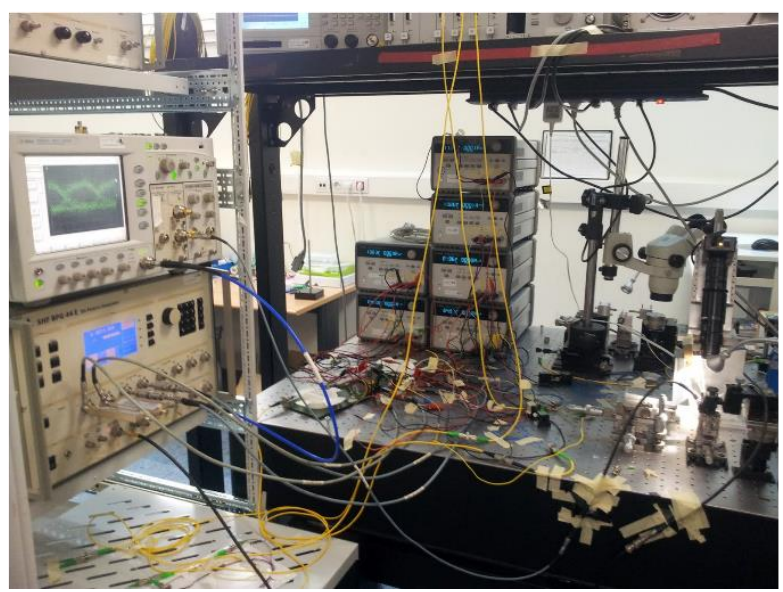

Figure 3.32: (a) Experimental setup for characterizing the DQPSK receiver. POL CON indicates polarization controller. (b) Photograph of a part of the set-up used to generate and measure the DQPSK demodulated signal.

The DQPSK transmitted eyes at 10 and $20 \mathrm{Gbit} / \mathrm{s}$ are shown in Figure 3.33, as well as the reference constellation obtained by using a discrete receiver described in [SOT12].
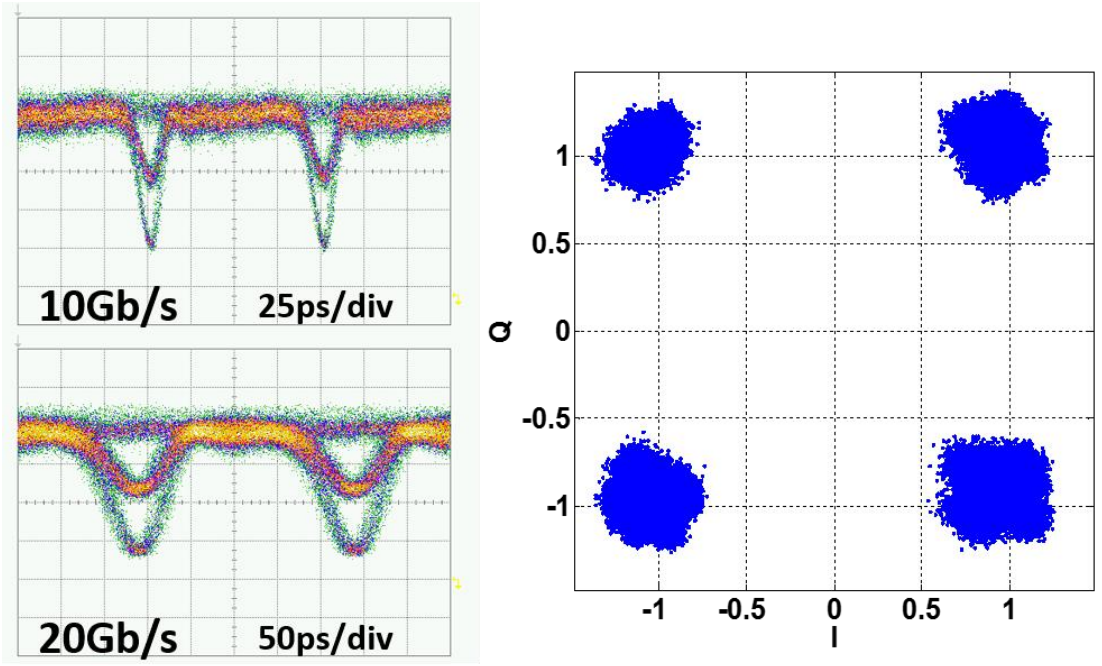

Figure 3.33: $10 \mathrm{Gbit} / \mathrm{s}$ and $20 \mathrm{Gbit} / \mathrm{s}$ transmitted DQPSK eyes, and reference constellation measured by a discrete receiver (20 Gbit/s). 
For the $10 \mathrm{Gbit} / \mathrm{s}$ integrated receiver, well opened eye-diagrams and remarkably good constellation diagrams were obtained, as shown in Figure 3.34 for a received power of $19 \mathrm{dBm}$. It must be commented that in long haul networks, the parameter of interest lies in the relation between the BER and the optical signal to noise ratio, due to the fact that the signal is usually degraded by noise introduced by a large number of amplifiers. However, in an access network, the figure of merit is the sensitivity as a function of received power, as there is no degradation due to amplification stages and the receiver sensitivity limits the possible splitting ratio and reach of the system [QIA11, JEN07].

In this point, a key parameter as the error vector magnitude (EVM) for performance measurements must be defined. The EVM metric, which is a standard in wireless and wireline communications, describes the effective distance of the received complex symbol from its ideal position in the constellation diagram.

EVM is defined as the root mean square of the error of every received symbol, normalized over the average power of all the symbols in the constellation [SCH12]. EVM is calculated by the following formula:

$$
E V M=\left[\frac{\frac{1}{T} \sum_{t=1}^{T}\left[\left(I_{r, t}+I_{t}\right)^{2}+\left(Q_{r, t}+Q_{t}\right)^{2}\right]}{\frac{1}{M} \sum_{m=1}^{M}\left(I_{m}^{2}+Q_{m}^{2}\right)}\right]^{\frac{1}{2}}
$$

where $I_{r, t}, Q_{r, t}$ are the in-phase and quadrature components of the $t^{\text {th }}$ received symbol, and $I_{m}, Q_{m}$ are the in-phase and quadrature components for the ideal $m^{\text {th }}$ symbol in the constellation. The denominator in Equation 3.10 is the average power of all the symbols of the DQPSK constellation, which normalizes the error metric.

The EVM as a function of the received power was measured and the experimental results are summarized in Table 3-2.

a)

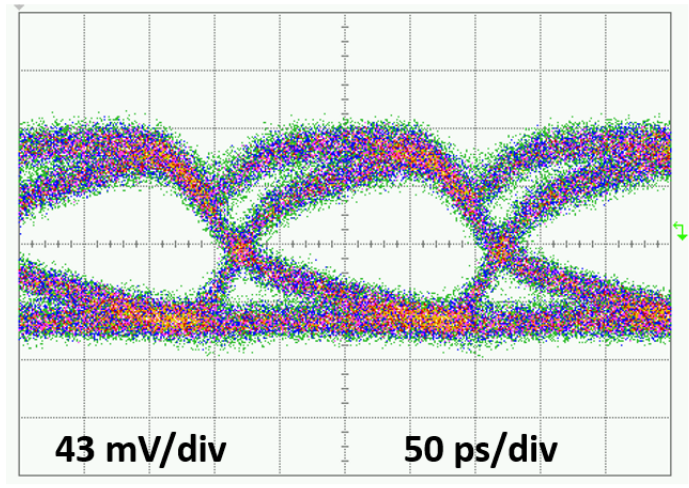

b)

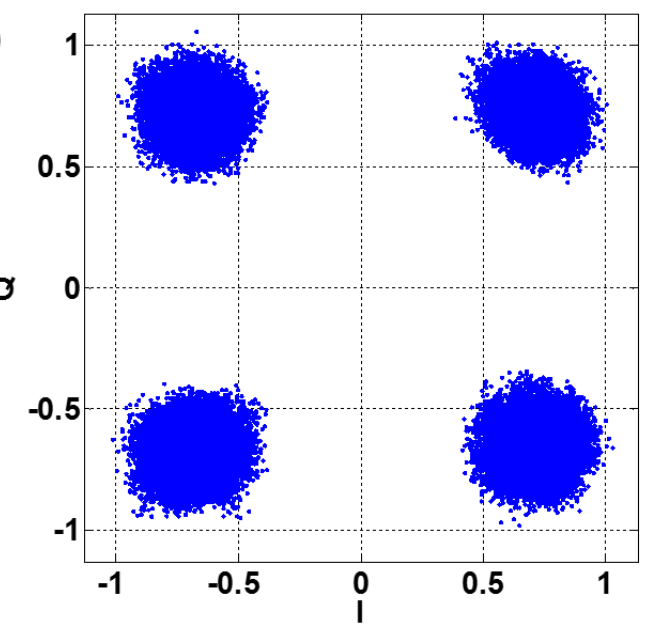

Figure 3.34: (a) DQPSK eye diagram and (b) symbol constellation at 10Gbit/s for a received power of $19 \mathrm{dBm}$. 
As the quality of the received signal was quite good so that no significant amount of errors were recorded in the captured length of the data, which was $100 \mathrm{k}$ symbols, the measured EVM of the signal was used to estimate the BER by using the following equation taken from [SHA06] and experimentally demonstrated in [SCH12]:

$$
B E R \approx \frac{\left(1-\frac{1}{L}\right)}{\log _{2} L} Q\left[\sqrt{\left.\left[\frac{3 \log _{2} L}{L^{2}-1}\right] \frac{\sqrt{2}}{E V M^{2} \log _{2} M}\right]}\right.
$$

where $L$ is the number of signal levels per quadrature, $M$ is the order of the modulation and $Q$ indicates the $\mathrm{Q}$ function. For QPSK modulation, $L$ is 2 and $M$ is 4 .

TABLE 3-2

EVM AND BER, FOR THE 10 GBIT/S DIFFERENTIAL RECEIVER.

\begin{tabular}{|c|c|c|}
\hline Received Power $(\mathrm{dBm})$ & Measured EVM $(\%)$ & Estimated BER \\
\hline-23 & 24.27 & $1.9 \times 10^{-5}$ \\
\hline-22 & 22.39 & $4 \times 10^{-6}$ \\
\hline-21 & 14.76 & $6.2 \times 10^{-12}$ \\
\hline-20 & 13.08 & $10^{-14}$ \\
\hline $\mathbf{- 1 9}$ & $\mathbf{1 2 . 5}$ & $\mathbf{1 0}^{-15}$ \\
\hline
\end{tabular}

a)

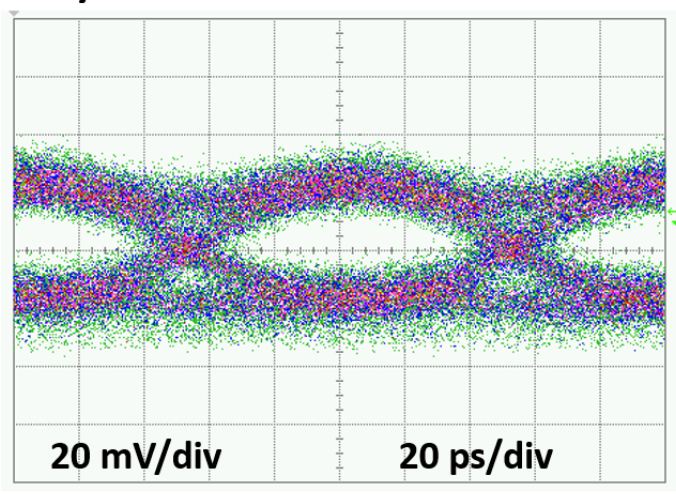

b)

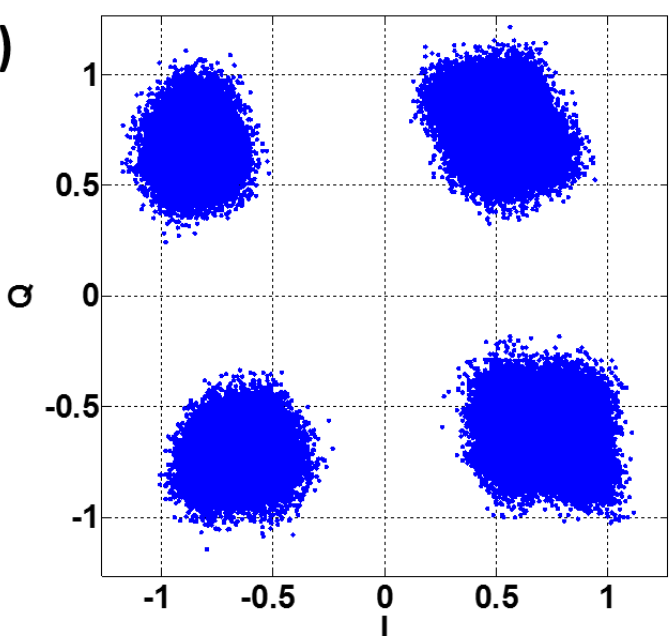

Figure 3.35: (a) DQPSK eye diagram and (b) symbol constellation at $20 \mathrm{Gbit} / \mathrm{s}$ for a received power of $12 \mathrm{dBm}$. 
Examining the results shown in the table above, an error floor value of around $12.5 \%$ and $10^{-15}$ were obtained for the EVM and BER respectively.

For the $20 \mathrm{Gbit} / \mathrm{s}$ DQPSK receiver, opened eyes and good constellation diagram were also measured, as shown in Figure 3.35 for a received power of $-12 \mathrm{dBm}$. Comparing the obtained constellation diagram with the reference one shown in Figure 3.33, it can be seen that some additional noise appears in the symbols of the constellations. Nevertheless, the EVM of the obtained constellation was measured to be $19.57 \%$ and the corresponding BER was estimated, by using Eq. 3.11, in $1.7 \cdot 10^{-7}$, as shown in Table 3-3. It must be taken into account that typically, advanced modulation formats operate with Forward-Error Correction (FEC), and our BER values, without FEC, are below the $10^{-3}$ reference, which is the FEC limit for 10G-Ethernet Passive Optical Network (EPON) standard [ITU987, BET10].

\section{TABLE 3-3}

EVM and BER, for the $20 \mathrm{Gbit} / \mathrm{s}$ differential receiver.

\begin{tabular}{|c|c|c|}
\hline Received Power $(\mathrm{dBm})$ & Measured EVM (\%) & Estimated BER \\
\hline-14 & 25.66 & $6 \times 10^{-5}$ \\
\hline-13 & 19.68 & $1.9 \times 10^{-7}$ \\
\hline $\mathbf{- 1 2}$ & $\mathbf{1 9 . 5 7}$ & $\mathbf{1 . 7}^{-7} \mathbf{1 0}^{-7}$ \\
\hline
\end{tabular}

A $40 \mathrm{Gbit} / \mathrm{s}$ DQPSK receiver was also measured at the NTC's laboratories. It must be commented that in these laboratories, balanced detection was not used but single-ended detection, and for both photodetectors (I, Q) clean eyes opening were obtained and are shown in Figure 3.36. Unfortunately, when these chips were brought to TU/e facilities to be characterized by using balanced detection in order to obtain the DQPSK constellations, the RF pads were damaged during the experiment.
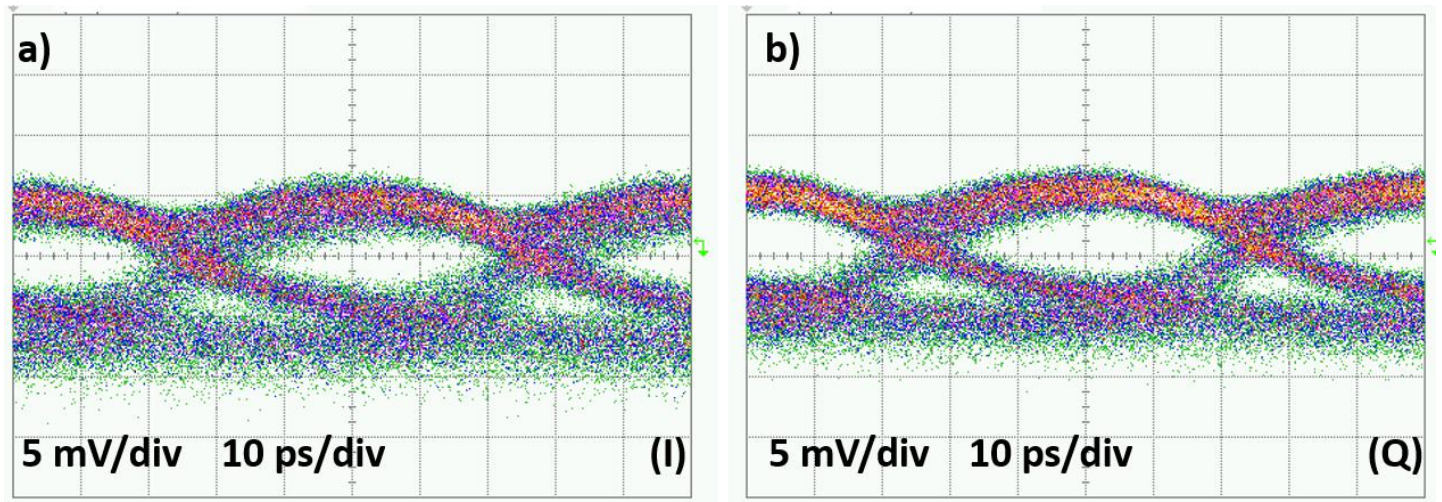

Figure 3.36: DQPSK eye diagram (a) In-phase and (b) Quadrature component at $40 \mathrm{Gbit} / \mathrm{s}$. 
At this point, we have demonstrated a minimum footprint differential receiver for DQPSK transmission with zero biased detection for access networks has been demonstrated at 10 and $20 \mathrm{Gbit} / \mathrm{s}$. Each building block of the receiver was optimized for high performance and maximum compactness. The results indicate the potential of integrated silicon receivers to become key building blocks for future passive optical access networks based on advanced modulation formats. The future work would be the integration of the receiver with transimpendance amplifiers would improve its sensitivity and so higher-order modulation formats could be supported due to the low EVM values achieved. For instance, for an EVM of 12.5\%, translated into BER for D8PSK, results into a BER lower than $10^{-5}$ [SCH12]. 


\section{Chapter 4}

\section{Conclusions and Future Outlook}

Future access networks need to provide broadband services using wired and wireless approaches. Passive optical network is promising for providing wired services while radio-over-fiber is an important technique for providing wireless services in the optical domain. Wireless access network play very important role in our daily lives by providing flexible network connections to users anytime and anywhere. Gigabit capacity wireless links are evolving at a very rapid pace and wireless links matching capacities of optical communication links are extensively investigated in order to bridge the existing bottle neck between the wired and wireless communication systems.

In this work, different application scenarios have been identified for the $\mathrm{mmW}$ wireless architecture, and the main functional building blocks involved in the 10 Gbps wireless transmission have been studied and experimentally demonstrated.

Regarding the transmitter chip, a tunable ring resonator based demultiplexer and a DPSK MZM had been designed, fabricated and characterized, demonstrating high performance for both devices. A successful demonstration of an error-free DPSK modulation at 5 Gbit/s and $10 \mathrm{Gbit} / \mathrm{s}$ using a dual-drive silicon MZM was reached. Furthermore, we have also shown the feasibility of the proposed MZM for $15 \mathrm{Gbit} / \mathrm{s}$ and $20 \mathrm{Gbit} / \mathrm{s}$ DPSK modulation and the obtained results validate the potential to achieve higher order modulation formats, such as QPSK, by arranging the MZM in a nested configuration.

On the receiver side, a DQPSK device in addition to a polarization diversity scheme have been presented. The transparent polarization behaviour is given by a polarization diversity scheme, where the main blocks are a polarization splitter and polarization rotator in order to separate the random orthogonal incoming polarizations and rotate the TM one. This scheme can be also used to duplicate the capacity system by introducing polarization multiplexing. The performance of the polarization splitter and rotator satisfy the requirements targets imposed in the HELIOS project. 
The DQPSK receiver included a thermo-optically tunable MZI power splitter, a MZDI, a 90 degree hybrid based on a multimode interference coupler and two balanced germanium photodetectors. Each building block of the receiver was optimized for high performance and maximum compactness and a minimum footprint differential receiver for DQPSK transmission with zero biased detection for access networks has been demonstrated at 10 Gbit/s and $20 \mathrm{Gbit} / \mathrm{s}$. Well opened eye-diagrams and symbol constellations were obtained with error vector magnitude values as low as $12.5 \%$ and $19.57 \%$ at $10 \mathrm{Gbit} / \mathrm{s}$ and 20 Gbit/s, respectively. The obtained results indicate the potential of integrated silicon receivers to become key building blocks for future passive optical access networks based on advanced modulation formats.

As future work, the packaging and testing of the integrated photonic DQPSK transmitter and receiver with integrated transimpedance amplifiers on a printed circuit board would improve its sensitivity and so higher-order modulation formats could be supported due to the low EVM values achieved. For instance, for an EVM of $12.5 \%$, translated into BER for D8PSK, results into a BER lower than $10^{-5}$ [SHC12]. Scaling the MZM's bit rate to even higher bit rates, and demonstrating DQPSK modulation are the next steps to be carried out, before packaging and testing the integrated transceiver. The figure below shows how our transmitter once it had been packaged.
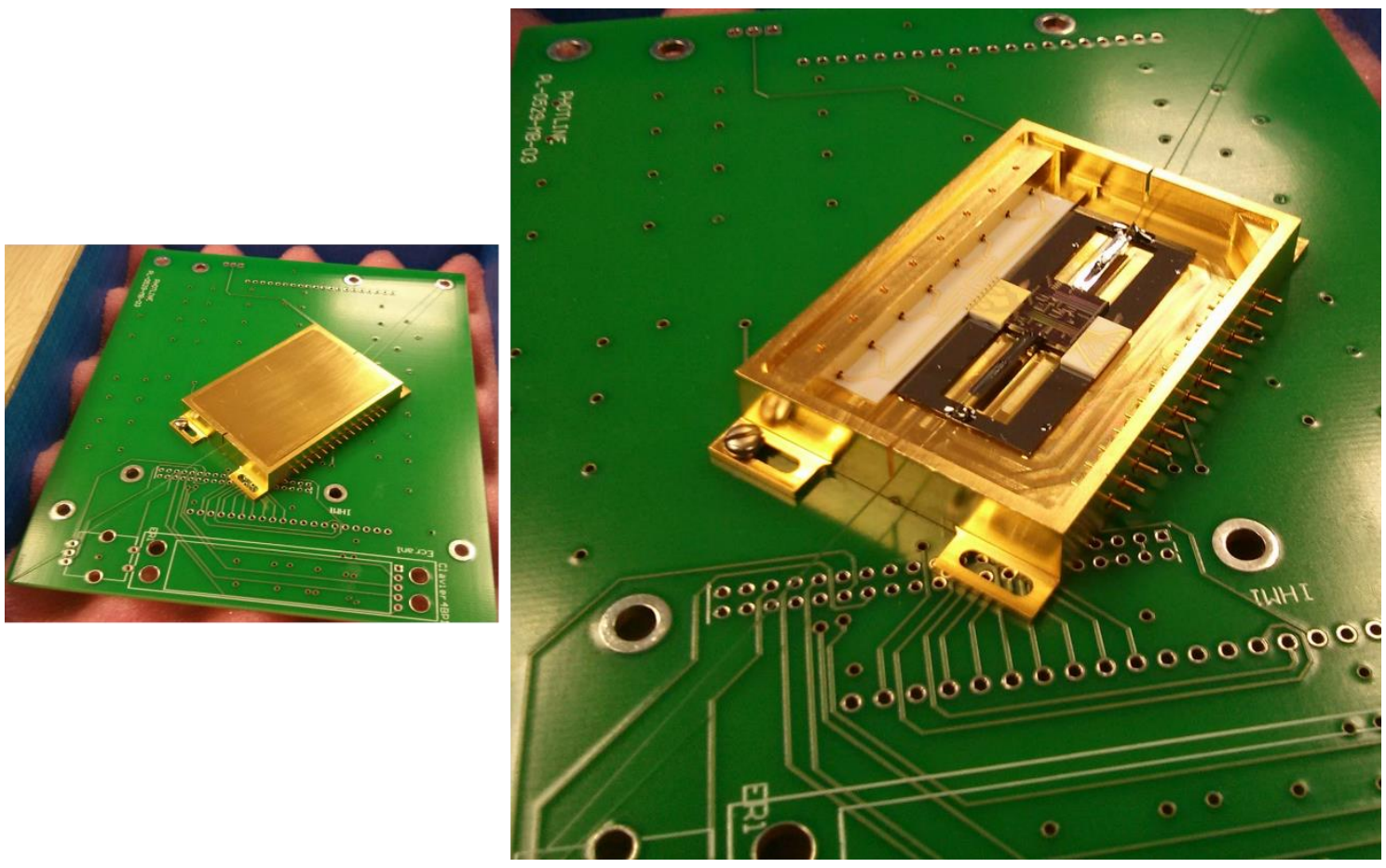

Figure 4.1: Photograph of the packaged transmitter. 


\section{Appendix A}

\section{A. Development process of photonic structures}

A complete development process of any photonic structure can be divided generally in basic four steps: design (including modelling, simulation and analysis), mask generation, fabrication and characterization. At this point, a broad terms description of each step will be carried out.

\section{Design}

When light propagation in optical integrated waveguides is referred to, two physical approaches which are presented in such mechanism must be considered: on one hand the ray optics approach or geometrical optics, which enables light propagation along an optical waveguide by total internal reflection [REE04] (in silicon this mechanism is highly effective due to the high index contrast effect); and on the other hand, the electromagnetic approach (by means of Maxwell's equations), which allows to derive the guided modes and the field patterns. Maxwell's equations enable to make a rigorous study of the mode profiles propagating along the considered waveguide.

First, the designer must try to model the performance of the device under study. The modeling of photonic structures may not always be carried out in an analytic way, especially when one deals with high index contrast structures with non-uniform refractive index profiles all over the propagation length. In that case, the device under study must be simulated to analyze its performance. 

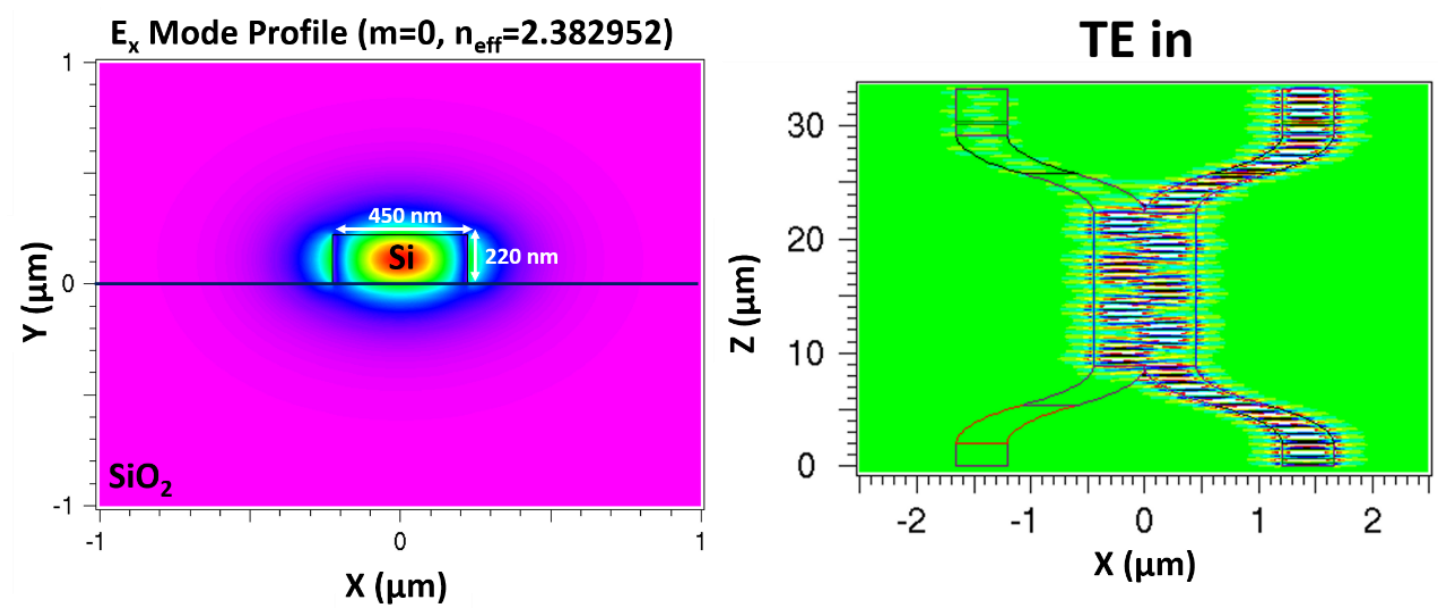

Figure A-1: (Left) BeamPROP simulation of the fundamental mode in a silicon waveguide of 220nm height and 450nm width for TE polarization, (Right) FullWAVE simulation multimode interference waveguide.

Several techniques exist to perform these simulations. Among them, some of those that have been employed to simulate the behaviour of our photonic structures are: the finite time difference domain (FDTD) method, which consists in discretizing Maxwell's equations via finite differences, the plane wave expansion (PWE) method, which, in contrast with FDTD, computes the electromagnetic field as a superposition of planes waves, which are straightforward solutions of Maxwell's equations in an uniform medium or beam propagation method (BPM) used for some structures which are uniform in the propagation direction. The simulation tools used in the Nanophotonics Technology Center to carry out the above mentioned methods are, respectively: FullWAVE, BandSOLVE and BeamPROP from RSOFT $^{\mathrm{TM}}$ [RSO00]. Figure A-1 depicts two simulation examples.

\section{Mask generation}

Once the designer is in possession of appropriate design parameters derived from the modelling and simulation process, he/she must carry out the mask design. When generating the mask layout, the designer should consider the specific lithography process limitations, essentially given by the wavelength of the illuminating radiation. Usually, a set of photonic structures are designed varying the parameters in a wide range. Moreover, each several repetitions of the same mask are positioned at different locations on the wafer since the deep-uv (used for lithography) incident dose, and therefore the waveguide width (as the resist is more exposed) depends on positioning on the wafer. 
In the Nanophotonics Technology Center, Raith150 lithography software it is used [RAI150]. This tool generates the GDS final file of the mask which, finally is sent to the fabrication foundry. The figure below shows an example of a GDS file.

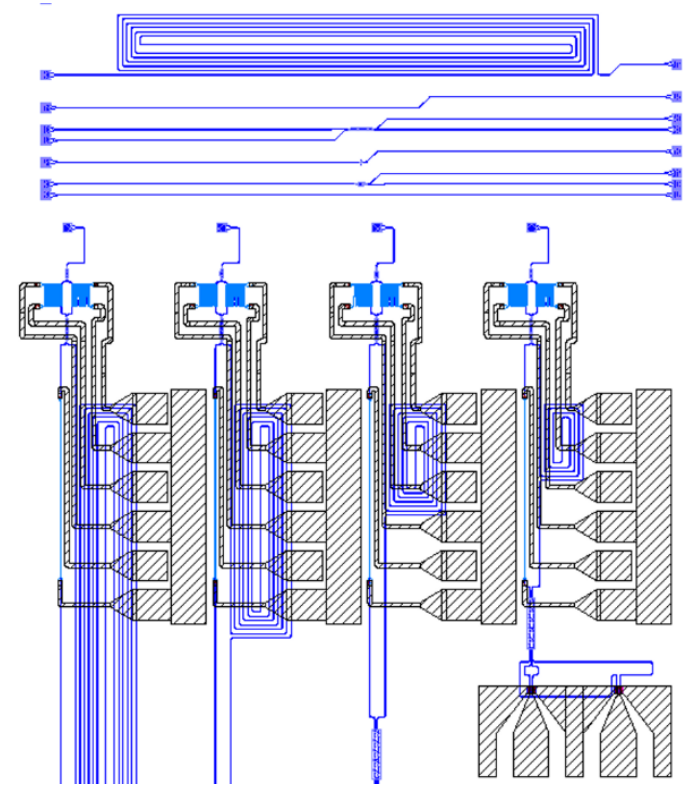

Figure A-2: Final GDSs mask layers of the some of the fabricated devices characterized in this thesis.

\section{Fabrication}

Today's trend in silicon photonics is demonstrating that any new device design may be mass produced in CMOS foundries. Such entities consist of advanced nanofabrication tools and highly experienced engineers. Working in a controlled environment gives the designer the opportunity to be aware of the reality of fabrication constraints and capabilities.

The complexity of active silicon devices requires numerous demanding fabrication steps, which must be handled cautiously to prevent time and money consuming errors. For the abovementioned reasons, a CMOS foundry provides a suitable platform to realize complex active silicon devices such. In Europe, the foundry model relies on ePIXfab [EPI] within the FP7 framework, which organizes wafer-scale fabrication services of silicon photonic integrated circuits. It consists of two institutions: CEA-LETI [MIN], IMEC [IME] and IHP [IHP], with similar standard fabrication capabilities summarized below. Design rules and guidelines are provided in the technology paper standard of both CEA-LETI and IMEC [EPI]. In addition to passive technology, ePIXfab offers ion implantation services to realize silicon active devices based on the plasma dispersion effect. The most common impurities used to dope silicon are boron and phosphorus, which are respectively situated in the III column and V column of the periodic table. Finally, silicide and metallization are also available processes to form low loss contacting electrodes. 
Specifically, the fabrication of all silicon-based modulators involved in this thesis was carried out at CEA-LETI on 8 inches wafer, with $193 \mathrm{~nm}$ deep-UV lithographic process.

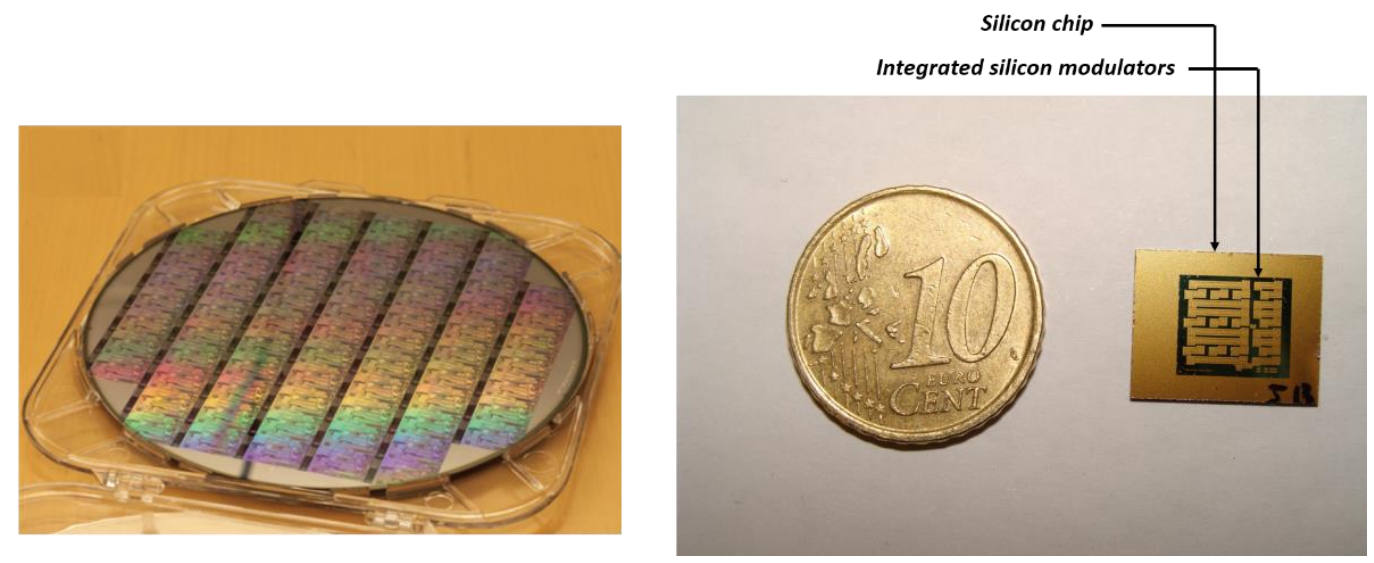

Figure A-3: (Left) Photograph of an 8-in SOI wafer with hundreds of photonic components and circuits and (Right) picture of a single silicon chip fabricated in CEA-LETI consisting of 26 electro-optical modulators.

\section{Characterization}

The main task of this thesis is centered at this step of the process. The characterization in the laboratory of all electro-optic silicon-based modulators developed and presented along the thesis has been carried out entirely at the NTC facilities. Several types on characterization have been performed: passive, RF, high speed, small signal. All of them are deeply explained in each section, as well as, the characterization set-up and the equipment used and obviously the obtained results. 


\section{List of Publications}

\section{Journal publications}

[J.1] M. Aamer, D.J. Thomson, A.M. Gutiérrez, A. Brimont, F.Y. Gardes, G.T. Reed, J.M. Fedeli, A. Hakansson, and P. Sanchis, "10Gbit/s error-free DPSK modulation using a push-pull dual-drive silicon modulator", Optics Communications, 304, pp. 107-110 (2013).

Doi: 10.1016/j.optcom.2013.04.051.

[J.2] M. Aamer, N. Sotiropoulos, A. Brimont, J.M. Fedeli, D. Marris-Morini, E. Cassan, L. Vivien, K. Ribaud, P. GROSSE, J. Hartmann, D. Vermeulen, G. Roelkens, P. Sanchis, and A. Hakansson, "A Silicon Differential Receiver with Zero Biased Balanced Detection for Access Networks," Photonics Technology Letters, IEEE., 25, pp. 1207-1210 (2013)

Doi: 10.1109/LPT.2013.2262931

[J.3] M. Aamer, A.M Gutierrez, A. Brimont, D. Vermeulen, G. Roelkens, J.M. Fedeli, A. Hakansson, and P. Sanchis, "CMOS Compatible Silicon-on-Insulator Polarization Rotator Based on Symmetry Breaking of the Waveguide Cross Section," Photonics Technology Letters, IEEE , 24, pp.2031-2034 (2012). Doi: 10.1109/LPT.2012.2218593.

[J.4] M. Aamer, A. Griol, A. Brimont, A. Gutierrez, P. Sanchis, and A. Håkansson, "Increased sensitivity through maximizing the extinction ratio of SOI delayinterferometer receiver for 10G DPSK," Optics Express, 20, pp. 14698-14704 (2012).

Doi 10.1364/OE.20.014698

[J.5] M. Aamer, A.M. Gutiérrez, A. Brimont, P. Sanchis, "Ring Resonator Structures for Active Applications in Silicon", Optica Pura y Aplicada, 45, pp. 169-175 (2012).

ISSN-e 2171-8814

\section{Conference publications}

[O.1] M. Aamer, A. Griol, A. Brimont, A. M. Gutierrez, P. Sanchis, and A. Håkansson, "Increased sensitivity of delay-interferometer receiver for 10G DPSK for SOI platform" in $3^{\text {rd }}$ Conferencia Española de Nanofotonica (2012). 
[O.2] M. Aamer, A. Brimont, A. M. Gutierrez, A. Griol, J. Martí, and P. Sanchis; "Optimized 20 Gbps DPSK demodulator in 220nm SOI." 16th European Conference on Integrated Optics and Technical Exhibition - ECIO (2012).

[O.3] M. Aamer, A. M. Gutierrez, A. Brimont, A. Griol, and P. Sanchis; "Experimental demonstration of an ultracompact SOI polarization rotator". 16th European Conference on Integrated Optics and Technical Exhibition - ECIO (2012).

[O.4] M. Aamer, A. M. Gutierrez, A. Brimont, and P. Sanchis, "Ring Resonator Structures for Active Applications in Silicon". $7^{\text {a }}$ Reunión Española de Optoelectrónica (2011). ISBN: 978-84-86116-31-6

[0.5] A. Hakansson, M. Aamer, N. Sotiropoulos, A. Brimont, P. Sanchis, J.M. Fedeli, D. Marris-Morini, E. Cassan, L. Vivien, K. Gilbert, P. Grosse, J.M. Hartmann, D. Vermeulen and G. Roelkens, "Compact Silicon Differential Receiver with Integrated Zero Biased Balanced Detection". In Group IV Photonics (GFP), 9th IEEE International Conference on, 165 - 167 (2012).

[O.6] P. Sanchis, M. Aamer, A. Brimont, A.M. Gutierrez, N. Sotiropoulos, H. de Waardt, D. J. Thomson, F. Y. Gardes, G. T. Reed, K. Ribaud, P. Grosse, J. M. Hartmann, J.-M- Fedeli, D. Marris-Morini, E. Cassan, L. Vivien, D. Vermeulen, G. Roelkens, and A. Hakansson, "Silicon CMOS photonics platform for enabling high-speed DQPSK transceivers”, ICTON 2013 (invited talk).

\section{Other publications}

[O.1] A. Brimont, A. M. Gutierrez, M. Aamer, D.J. Thomson, F. Y. Gardes, J.M Fedeli, G. T. Reed, J. Martí, and P. Sanchis, "Slow-Light-Enhanced Silicon Optical Modulators Under Low-Drive-Voltage Operation”, IEEE Photonics Journal, 4, pp. 1306-1315 (2012).

[O.2] A. M. Gutierrez, A. Brimont, J. Herrera, M. Aamer, J. Martí,D. Thomson, F.Y. Gardes, G. T. Reed, J. M. Fedeli, and P. Sanchis, "Silicon slow-light-based photonic mixer for microwave-frequency conversion applications". OPTICS LETTERS, 37, pp. 1721-1723 (2012).

[O.3] A. M. Gutierrez, A. Brimont, M. Aamer, and P. Sanchis; "Method for measuring waveguide propagation losses by means of a Mach-Zehnder Interferometer structure". OPTICS COMMUNICATIONS, 285, pp. 1144-1147 (2012).

[O.4] A. Brimont, A. M. Gutierrez, M. Aamer, D.J. Thomson, F.Y. Gardes,J.-M. Fedeli, G.T. Reed, J. Martí, and P. Sanchis, "Recent progress on high contrast $40 \mathrm{~Gb} / \mathrm{s}$ and low drive voltage $10 \mathrm{~Gb} / \mathrm{s}$ slow light-based silicon photonic modulators", in $3^{\text {rd }}$ Conferencia Española de Nanofotonica (2012).

[O.5] A. M. Gutiérrez, J. V. Galan, J. Herrera, A. Brimont, M. Aamer, J. Martí, D. Marris-Morini, L. Vivien, J-M. Fédéli, D. J. Thomson, F. Y. Gardes, G. T. Reed, and P. Sanchis, "Silicon-Based Electro-Optic Modulators for Linear and Nonlinear Radio-over-Fiber Applications". Accepted in IEEE International Topical Meeting on Microwave Photonics, 168-171 (2012).

[O.6] A. M. Gutierrez, A. Brimont, M. Aamer, J. Martí, P. Sanchis, D. Thomson, F.Y. Gardes, G. T. Reed, and J. M. Fedeli; “A slow light silicon-based Mach-Zehnder 
Interferometer forfrequency mixing applications". 16th European Conference on Integrated Optics and Technical Exhibition - ECIO (2012).

[O.7] A. Rosa, A. M. Gutiérrez, A. Brimont, A. Aamer, and P. Sanchis, "A siliconbased tunable multimode interferometer using the thermo-optic effect". 16th European Conference on Integrated Optics and Technical Exhibition - ECIO (2012).

[O.8] Galan, J.V.; Aamer, M.; Sanchis, P.; Griol, A.; Bellieres, L.; Ayucar, J.; and Marti, J., "A compact and broadband polarization splitter in SOI," LEOS Annual Meeting Conference Proceedings (LEOS '09). IEEE, 309-310 (2009). doi: 10.1109/LEOS.2009.5343249

[0.9] A. M. Gutierrez, A. Brimont, J. Herrera, M. Aamer, D. J. Thomson, F. Y. Gardes, G. T. Reed, J-M. Fédéli, and P. Sanchis, "Modeling of the nonlinear distortion in an electro-optic silicon-based Mach-Zehnder modulator", Accepted in Group IV Photonics (GFP), 10th IEEE International Conference on, (2013). 


\section{List of Figures}

Figure 1.1: Aspect of the fundamental mode in a rectangular waveguide for the TE (left) and TM (right) polarization, and the values of the effective indices, resulting $\Delta n_{\text {eff }} \approx 0.57$

Figure 1.2: Artistic projection of the various application scenario of a gigabit wireless

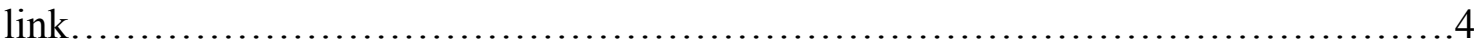

Figure 1.3: Illustration of a millimeter wave transmitter...........................6

Figure 1.4: Infinera's 10 and 40-channel transmitter and receiver integrating SOAs......7

Figure 1.5: (a) Luxtera's photograph of CMOS 4x10Gb/s WDM die (b) on flexible circuit and fiber-array. (c) Luxtera integrations scheme for transceiver $4 \times 10 \mathrm{~Gb} / \mathrm{s} \ldots \ldots \ldots \ldots \ldots . . . .8$

Figure 1.6: Picture of Intel's $50 \mathrm{~Gb} / \mathrm{s}$ transmitter and receiver. The transmitter includes hybrid IIIV/SOI lasers using wafer bonding and a wavelength multiplexer, the receiver includes a wavelength demultiplexer and $\mathrm{Ge} / \mathrm{Si}$ photodetectors......................9

Figure 1.7: Kotura's 4x25Gb/s transmitter and receiver chips...................... 9

Figure 1.8: (Left) Chip microphotograph of the $25 \mathrm{~Gb} / \mathrm{s}$ optical transceiver. At the bottom, waveguide couplers are shown on the same silicon wafer. Two millimetres between the couplers and the active circuits is omitted. (Right) an infrared image of the chip shows light leakage from silicon photonic waveguide and absorption in ring modulator. .10

Figure 1.9: Block diagram of the photonic integrated (Up) transmitter (Down) receiver circuits

Figure 2.1: Schematic of the integrated millimetre wave transmitter.

Figure 2.2: The four types of waveform keying with binary coding

Figure 2.3: Classification of intensity and phase modulation formats for optical communications nowadays................................................... 16

Figure 2.4: Principle of DPSK modulation generation using a MZM................... 17 
Figure 2.5: Symbol diagrams, normalized to unity average optical power, for a) OOK modulation (dashed lines represent examples of transitions between symbols for chirped formats). b) DPSK modulation (dashed double-arrows represent different phase modulator implementation). c) DQPSK modulation .................................17

Figure 2.6: Phase modulation can either be achieved using an MZM or by means of a straight-line PM, resulting in different intensity and phase waveforms................ 18

Figure 2.7: Structure of a DQPSK transmitter. Two MZMs are used as phase modulators, and the two separately modulated fields are combined with a $\pi / 2$ phase shift. Constellations and eye diagrams are also shown.

Figure 2.8: Optical spectra and optical intensity eye diagrams for (Left) NRZ-DPSK and (Right) NRZ-DQPSK

Figure 2.9: Q-factor of the signal as function of the bit error rate (BER), for QPSK modulation. Dashed line indicates $\mathrm{BER}=10^{-9}$, corresponding to a Q-factor of 15.6 $\mathrm{dB}$

Figure 2.10: Dependence of the transmitter optical filter and the Q-factor of the signal .21

Figure 2.11: Q-factor with respect to the ER achieved in the modulated port (DQPSK Arm) for different values of ER in the un-modulated port $(\Delta$-blue, $\bigcirc$-red and $\square$-black line).

Figure 2.12: Phase (black dashed) and module of the amplitude (blue solid) dependence of a single-arm-driven MZM as function of applied voltage to arm2, normalized by $V_{\pi}$ .23

Figure 2.13: Phase (black dashed) and amplitude module (blue solid) variation of a MZM in push-pull configuration.

Figure 2.14: SOI waveguide with an estimated $n_{\text {eff }}$ of 2.38. (Left) SEM image and (Right) a mode profile calculation .24

Figure 2.15: Design parameters for a ring resonator .26

Figure 2.16: Model of a single ring resonator with one waveguide.... .26

Figure 2.17: Schematic of an add-drop microring resonator with (a) straight couplers and (b) interferometric couplers

Figure 2.18: Shift of the resonant wavelength due to a shift of the effective index 32

Figure 2.19: (a) Add-drop filter using a single tuned ring resonator where only the coupling through port is tuned as well as the phase. The red striped layer corresponds to 
the microheater metallization, the black striped corresponds to the pads and the blue to the optical waveguides. (b) A close-up of the single-tuned RR filter.

Figure 2.20: (a) Add-drop filter using a double tuned ring resonator where both through and drop are tuned, as well as the phase. The red striped layer corresponds to the microheater metallization, the black striped corresponds to the pads and the blue to the optical waveguides. (b) A close-up of the double-tuned ring resonator filter.

Figure 2.21: (Left) Tuning of $\kappa$ with heater power: the dashed line marks the critical coupling level; (I), (II), (III), (IV) and (V) mark the states of initial, maximal, critical, minimal and critical coupling, respectively. (Right) Corresponding through (solid lines) and drop (dotted lines) ports spectra.

Figure 2.22: Tuning both coupling coefficients in order to achieve a tunable bandwidth while maintain a high extinction ratio. (Up) Through (Down) Drop ports spectra. The dotted line corresponds to $-3 \mathrm{~dB}$ transmission

Figure 2.23: (a) Schematic and (b) SEM image of the fabricated device. Heaters 1, 2 and 3 are used for tuning the coupling coefficient for through port, drop port, and wavelength,

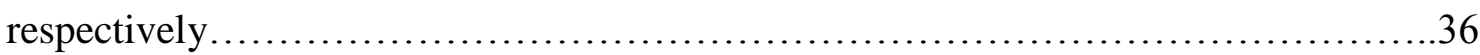

Figure 2.24: Image of the setup used to characterize the tunable DEMUX, using DC probes.

Figure 2.25: The control of the wavelength shift of the demultiplexer filter. The 5 graphs correspond to different applied voltage to the heater; from left to right, $0 \mathrm{~V}$ ( $\Delta$-green), $3 \mathrm{~V}$ ( $\square$-purple), 4V ( $\diamond$-pink), 5V ( ○-cyan) and 6V ( $\nabla$-black) respectively.

Figure 2.26: The transmission spectra for the through port of the demultiplexer when modifying the through coupling coefficient by applying different voltages to the heater. .38

Figure 2.27: The transmission spectra for the drop port of the demultiplexer when modifying the coupling coefficient to the through waveguide by applying different voltages to the heater.

Figure 2.28: (Up) Drop (dashed line) and through curves without any tuning mechanism. (Down) Tuned drop (dashed line) and through curves, where the specification values are almost achieved.

Figure 2.29: BER as function of the optical signal to noise ratio (a) $44.6 \mathrm{~Gb} / \mathrm{s}$ MZMDQPSK presented in [GOI13b]. (b) 56-Gb/s microring-QPSK

Figure 2.30: DPSK modulator. (a) GDS design, (b) optical photograph of fabricated device and (c) cross-section of the pn junction.

Figure 2.31: Normalized transmission spectra of the MZM with different bias voltages. 
Figure 2.32: Schematic of the experimental set-up for evaluating the performance of the DPSK modulator.

Figure 2.33: Images of some of the equipment used for the experimental characterization of the DPSK modulator

Figure 2.34: modulated DPSK eye diagram for (a) $5 \mathrm{Gbit} / \mathrm{s}$ (b) $10 \mathrm{Gbit} / \mathrm{s}$ (c) $15 \mathrm{Gbit} / \mathrm{s}$ and (d) $20 \mathrm{Gbit} / \mathrm{s}$

Figure 2.35: DPSK demodulated (a) AMI and (b) DB eye diagrams for 5 Gbit/s. AMI

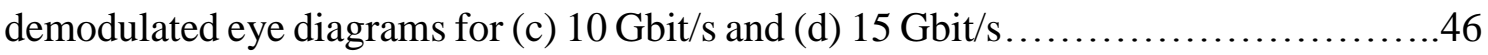

Figure 2.36: BER versus received power for 5Gbit/s ( $\odot-b l u e ~ c u r v e)$ and $10 \mathrm{Gbit} / \mathrm{s}(\diamond$-red curve) DPSK demodulation....................................................47

Figure 3.1: Polarization diversity scheme..................................52

Figure 3.2: Self-image formation along a multimode waveguide showing single and two-

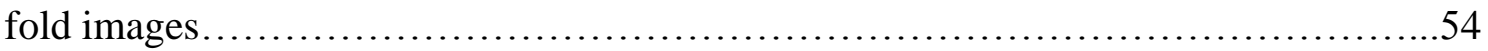

Figure 3.3: Proposed SOI polarization splitter.................................55

Figure 3.4: Theoretical normalized output power at a) port 3 and b) port 4 for TE and TM

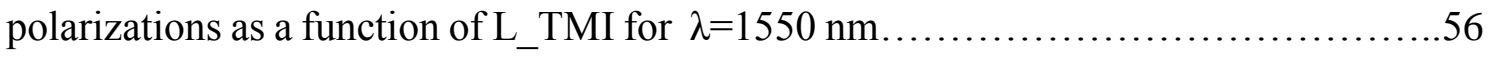

Figure 3.5: 3D-FDTD simulation capture for an input (Left) TE, and (Right) TM, polarization........................................................57

Figure 3.6: SEM images of a) fabricated polarization splitter and b) detail of the coupling

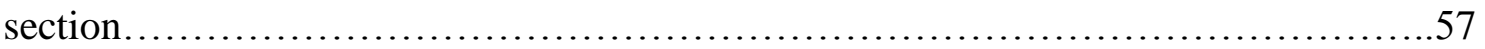

Figure 3.7: (a) Image of the whole experimental set-up used for the polarization splitter characterization. (b) Fiber coming from laser to input PC. (c) Translation stages and microscope. (d) Input lensed fiber, sample and objective lens. (e) Output polarized lens, power splitter lens, photodetector and IR camera.................................58

Figure 3.8: Block diagram of the setup for polarization splitter experimental measurements............................................................. 58

Figure 3.9: (a) Light spot captured by the infrared camera after proper alignment between the fiber, sample and objective lens. (b) Piezoelectric controller.

Figure 3.10: Normalized output power and theoretical and experimental extinction ratio for fabricated devices, for (a) bar and (b) cross ports.............................6

Figure 3.11: Measured output power spectrum at a) bar and b) cross ports..............60

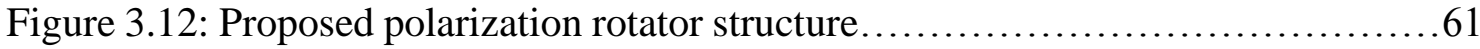


Figure 3.13: (a) Optical axes rotation after propagating along Lc. (b) simulated excitation of hybrid modes as a function of the waveguide width of the proposed polarization rotator, at the symmetric-asymmetric interface.

Figure 3.14: Polarization conversion efficiency (PCE) as a function of the polarization

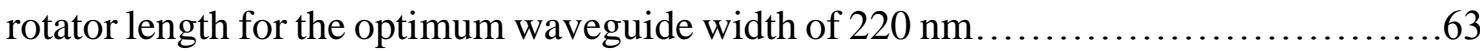

Figure 3.15: Polarization rotator robustness investigation as a function of the fill factor and etch depth. The contour lines are the PCE penalties with respect to the highest PCE of the device.

Figure 3.16: (a) Schematic of the whole polarization diversity structure used to characterize the polarization rotator. SEM images of (b) TE input focusing grating coupler, (c) polarization rotator, (d) polarization splitter and (e) TM output grating coupler.

Figure 3.17: Block diagram of the setup for polarization rotator experimental measurements.

Figure 3.18: Normalized measured spectra as a function of wavelength of polarization rotators with three different lengths

Figure 3.19: Polarization conversion efficiency as a function of wavelength for the

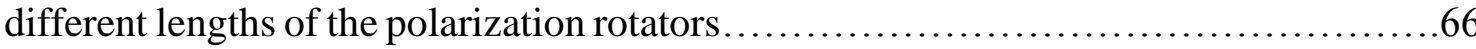

Figure 3.20: Schematic of a MZDI structure 68

Figure 3.21: Schematic of the proposed DPSK demodulator. From left to right in the figure: Two input TE grating couplers with waveguide tapers, $2 \times 2$ unbalanced MZI switch, MZDI with a $10 \mathrm{GHz}$ DL, a 2x1 MMI, taper, and an output grating coupler.....69

Figure 3.22: Simulated spectral response at the MZDI inputs (green-dotted, red-dashed) and at the demodulator output (blue-solid), taking into account $6 \mathrm{~dB} / \mathrm{cm}$ propagation loss

Figure 3.23: Optical photo of the MZI-MZDI structure. Below the left and right inset show a SEM image of the MZI with and MZDI spirals, respectively...

Figure 3.24: Measured spectral response at the DPSK demodulator. (a) Transmission spectrum of the full experimental wavelength band. The arrows mark the different states of the MZI switch at the input of the MZDI. (b) A zoom with 1pm resolution on the wavelength range where resonances have higher ER. Two resonances are marked in the figure: R1 for $16 \mathrm{~dB}$ ER and R2 with optimized $28 \mathrm{~dB}$ ER ...........................72

Figure 3.25: ER variation for different powers applied to the micro-heater on the MZI switch. The inset shows an optical image of the microheaters on top of the MZI

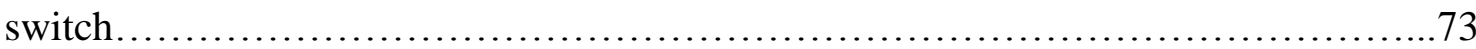

Figure 3.26: Experimental set-up used for the BER measurements...................73 
Figure 3.27: System measurements at $10 \mathrm{Gbps}$ of the DPSK demodulator. (a) BER measurements for the two studied resonances, R1 (red) and R2 (blue), marked in Figure 3.24. (b-c) the corresponding eye diagrams for $\mathrm{R} 1$ and $\mathrm{R} 2$ respectively................74

Figure 3.28: Schematic of a differential receiver with balanced detection..............75

Figure 3.29: DQPSK differential receiver. (a) GDS design and (b) optical photograph of fabricated device. The receiver is assembled using 5 components, from left to right in the figure: curved grating coupler, MZI tunable power splitter, DLI, $90^{\circ}$ hybrid and two balanced PDs .77

Figure 3.30: The simulated output of the receiver. The two curves represent how the inphase and quadrature detected current from the two diodes varies with the differential phase. The four different two-bits states are marked as dotted lines at $-90,0,90$, and 180 degrees, corresponding to $[+1+1],[-1+1],[-1-1]$, and $[+1-1]$, respectively $\ldots \ldots \ldots \ldots . . .78$

Figure 3.31: (a) The Dark (solid blue) and Light (dashed red) current measured for the fabricated photo diodes. (b)Output current as a function of wavelength for the $10 \mathrm{Gbit} / \mathrm{s}$ (solid lines) and $20 \mathrm{Gbit} / \mathrm{s}$ (dashed lines) bit rate receiver. The blue and the red lines correspond to the in-phase (I) and quadrature (Q) outputs, respectively.................79

Figure 3.32: (a) Experimental setup for characterizing the DQPSK receiver. POL CON indicates polarization controller. (b) Photograph of a part of the set-up used to generate and measure the DQPSK demodulated signal... .80

Figure 3.33: $10 \mathrm{Gbit} / \mathrm{s}$ and $20 \mathrm{Gbit} / \mathrm{s}$ transmitted DQPSK eyes, and reference constellation

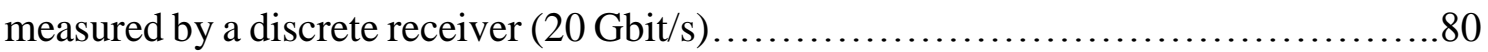

Figure 3.34: (a) DQPSK eye diagram and (b) symbol constellation at 10Gbit/s for a

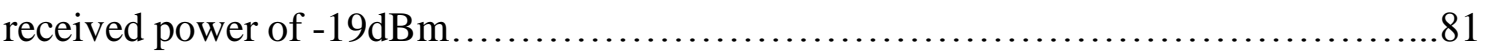

Figure 3.35: (a) DQPSK eye diagram and (b) symbol constellation at $20 \mathrm{Gbit} / \mathrm{s}$ for a

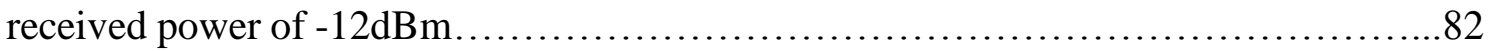

Figure 3.36: DQPSK eye diagram (a) In-phase and (b) Quadrature component at $40 \mathrm{Gbit} / \mathrm{s}$

Figure 4.1: Photograph of the packaged transmitter. .86

Figure A-1: (Left) BeamPROP simulation of the fundamental mode in a silicon waveguide of 220nm height and 450nm width for TE polarization, (Right) FullWAVE simulation multimode interference waveguide. . .88

Figure A-2: Final GDSs mask layers of the some of the fabricated devices characterized in this thesis.

Figure A-3: (Left) Photograph of an 8-in SOI wafer with hundreds of photonic components and circuits and (Right) picture of a single silicon chip fabricated in CEA-LETI consisting of 26 electro-optical modulators. . .90 


\section{List of Tables}

TABLE 2-1: Building blocks and HELIOS performance requirements for millimetre wave transmitter........................................................ 24

TABLE 3-1: Building blocks and HELIOS performance requirements for DQPSK receiver........................................................... 51

TABLE 3-2: EVM and BER, for the $10 \mathrm{Gbit} / \mathrm{s}$ differential receiver.

TABLE 3-3: EVM and BER, for the $20 \mathrm{Gbit} / \mathrm{s}$ differential receiver. .83 


\section{Acronyms}

$\begin{array}{ll}\text { AMI } & \text { Alternate-Mark Inversion } \\ \text { ASK } & \text { Amplitude Shift Keying } \\ \text { AWG } & \text { Arrayed Waveguide Grating } \\ \text { BER } & \text { Bit Error Ratio } \\ \text { BOX } & \text { Buried Oxide } \\ \text { BPD } & \text { Balanced Photodetector } \\ \text { BPG } & \text { Bit Pattern Generator } \\ \text { BWA } & \text { Broadband wireless access } \\ \text { CMOS } & \text { Complementary Metal-Oxide-Semiconductor } \\ \text { CSRZ } & \text { Carrier-Suppressed Return-to-Zero } \\ \text { DB } & \text { Duo Binary } \\ \text { DC } & \text { Direct Current } \\ \text { DCA } & \text { Digital Communication Analyzer } \\ \text { DGD } & \text { Differential Group Delay } \\ \text { DL } & \text { Delay Line } \\ \text { DQPSK } & \text { Differential Quadrature Phase Shift Keying } \\ \text { DSP } & \text { Digital Signal Processing } \\ \text { DWDM } & \text { Dense Wavelength-Division Multiplexing } \\ \text { ECL } & \text { Electro-Absorption } \\ \text { EXrnal Cavity Laser }\end{array}$




\begin{tabular}{|c|c|}
\hline EDFA & Erbium-Doped Fiber Amplifier \\
\hline ER & Extinction Ratio \\
\hline EVM & Error Vector Magnitude \\
\hline FDTD & Finite Difference Time Domain \\
\hline FEC & Forward Error Correction \\
\hline FFE & Feed-Forward Equalizer \\
\hline FSK & Frequency Shift Keying \\
\hline FSR & Free Spectral Range \\
\hline FTTH & Fiber-To-The-Home \\
\hline FWHM & Full Width at Half Maximum \\
\hline Gbps & Giga Bit Per Second \\
\hline GDS & Graphic Database System \\
\hline Ge-PD & Germanium Photo-Detector \\
\hline GPIB & General Purpose Interface Bus \\
\hline HELIOS & pHotonics ELectronics functional Integration on CMOS \\
\hline HSQ & Hydrogen SilsesQuioxane \\
\hline HTO & High Temperature Oxide \\
\hline ICP & Inductive Coupled Plasma \\
\hline IL & Insertion Loss \\
\hline IR & InfraRed \\
\hline LAN & Local Area Networks \\
\hline $\mathrm{LO}$ & Local Oscillator \\
\hline MLSE & Maximum Likelihood Sequence Estimation \\
\hline MMI & Multimode Interference \\
\hline $\mathrm{mmW}$ & millimeter wave \\
\hline $\mathrm{MZ}$ & Mach-Zehnder \\
\hline MZDI & Mach-Zehnder Delay Interferometer \\
\hline
\end{tabular}




\begin{tabular}{|c|c|}
\hline MZI & Mach-Zehnder Interferometer \\
\hline MZM & Mach-Zehnder Modulator \\
\hline NRZ & Non Return to Zero \\
\hline NTC & Nanophotonics Technology Center \\
\hline OFC/NFOEC & $\begin{array}{l}\text { Optical Fiber Communication Conference and Exposition and National Fiber } \\
\text { Optic Engineers Conference }\end{array}$ \\
\hline $\mathrm{OOK}$ & On-Off Keying \\
\hline OSNR & Optical Signal to Noise Ratio \\
\hline PBS & Polarization Beam Splitter \\
\hline $\mathrm{PC}$ & Polarization controller \\
\hline PCE & Polarization Conversion Efficiency \\
\hline PECVD & Plasma-Enhanced Chemical Vapour Deposition \\
\hline $\mathrm{PhC}$ & Photonic Crystal \\
\hline PIC & Photonic Integrated Circuits \\
\hline PLC & Planar Lightwave Circuit \\
\hline PM & Phase Modulator \\
\hline PMD & Polarization Mode Dispersion \\
\hline PolSK & Polarization Shift Keying \\
\hline PR & Polarization Rotator \\
\hline PRBS & Pseudo-Random Binary Sequence \\
\hline PSK & Phase Shift Keying \\
\hline QSFP & Quad Small Form-factor Pluggable \\
\hline RIE & Reactive Ion Etching \\
\hline ROSA & Receiver Optical Sub-Assembly \\
\hline RR & Ring Resonator \\
\hline RTD & Research, Technology and Development \\
\hline $\mathrm{RZ}$ & Returno to Zero \\
\hline
\end{tabular}


SEM

SOA

SOI

SSB

TE

TM

TMI

TOSA

UV

VLSI

VOA

WDM
Scanning Electron Microscope

Semiconductor Optical Amplifier

Silicon-On-Insulator

Single Side-Band

Transversal Electric

Transversal Magnetic

Two-Mode Interference

Transmitter Optical Sub-Assembly

UltraViolet

Very Large Scale of Integration

Variable Optical Attenuator

Wavelength-Division Multiplexing 


\section{Bibliography}

[AAM12] Aamer, M., Griol, A., Brimont, A., Gutierrez, A. M., Sanchis, P.; and Håkansson, A., "Increased sensitivity through maximizing the extinction ratio of SOI delay-interferometer receiver for 10G DPSK," Optics Express, 20(13), 14698. (2012). doi:10.1364/OE.20.014698

[AAM13a] Aamer, M., Thomson, D.J., Gutiérrez, A.M., Brimont, A., Gardes, F.Y., Reed, G.T., Fedeli, J.M.; Hakansson, A.; and Sanchis, P., "10Gbit/s error-free DPSK modulation using a push-pull dual-drive silicon modulator," Optics Communications, 304, 107-110, (2013). doi: 10.1016/j.optcom.2013.04.051

[AAM13b] Aamer, M.; Sotiropoulos, N.; Brimont, A.; Fedeli, J.M.; Marris-Morini, D.; Cassan, E.; Vivien, L.; Ribaud, K.; Grosse, P.; Hartmann, J.; Vermeulen, D.; Roelkens, G.; Sanchis, P.; and Hakansson, A., "A Silicon Differential Receiver with Zero Biased Balanced Detection for Access Networks," Photonics Technology Letters, IEEE., 25 (13), 1207 1210 (2013). doi: 10.1109/LPT.2013.2262931

[AGR02] Agrawal, G. P., "Fiber-Optical Communication Systems," 3rd Edition (Wiley-Interscience, 2002). doi: 10.1002/0471221147

[ALF09] Alfiad, M. S.; Van den Borne, D., Jansen, S. L., Wuth, T., Kuschnerov, M., Grosso, G., Napoli, A.; and De Waardt, H., "A Comparison of Electrical and Optical Dispersion Compensation for $111-\mathrm{Gb} / \mathrm{s}$ POLMUX-RZ-DQPSK," Lightwave Technology, Journal of, 27(16), 3590-3598, (2009). doi:10.1109/JLT.2009.2023534

[ARM06] Armani, A. M.; and Vahala, K. J. (2006). "Heavy water detection using ultra-high-Q microcavities," Optics Letters, 31(12), 1896-1898, (2006). doi:10.1364/OL.31.001896

[BAR07] Barwicz, T., Watts, M. R., Popović, M. A., Rakich, P. T., Socci, L., Kärtner, F. X.; Ippen, E. P.; and Smith, H. I, "Polarization-transparent 
microphotonic devices in the strong confinement limit," Nature Photonics, 1(1), 57-60, (2007). doi:10.1038/nphoton.2006.41

[BET10] De Betou, E. I., Mobilon, E., Angeli, B., Ohlen, P., Lindstrom, A., Dahlfort, S.; and Trojer, E., "Upstream FEC performance in combination with burst mode receivers for next generation $10 \mathrm{Gbit} / \mathrm{s}$ PON", Optical Communication (ECOC 2010), 36th European Conference and Exhibition on , 1-3, (2010). doi:10.1109/ECOC.2010.5621533

[BET92] Betti, S.; De Marchis, G.; Iannone, E., "Polarization modulated direct detection optical transmission systems," Lightwave Technology, Journal of, 10 (12), 1985-1997, (1992). doi: 10.1109/50.202809

[BIG04] S. Bigo, G. Charlet, and E. Corbel, "What has hybrid phase/intensity encoding brought to $40 \mathrm{Gbit} / \mathrm{s}$ ultralong-haul systems?", in proceedings of Optical Communication (ECOC 2004), 30th European Conference and Exhibition on, 872 - 875 (2004), Paper Th2.5.1

[BOS04] Bosco, G.; Carena, A.; Curri, V.; Gaudino, R.; Poggiolini, P., "Modulation formats suitable for ultrahigh spectral efficient WDM systems," Selected Topics in Quantum Electronics, IEEE Journal of, 10 (2), 321,328 (2004). doi: 10.1109/JSTQE.2004.827830

[BRI12] Brimont, A; Thomson, D. J., Gardes, F. Y., Fedeli, J. M., Reed, G. T.; Martí, J.; and\& Sanchis, P., "High-contrast $40 \mathrm{~Gb} / \mathrm{s}$ operation of a 500 $\mu \mathrm{m}$ long silicon carrier-depletion slow wave modulator," Optics Letters, 37(17), 3504-3506 (2012). doi:10.1364/OL.37.003504

[BRO06] Brooks, C; Jessop, P.; Deng, H.; Yevick, D.; and Tarr, G., "Passive silicon-on-insulator polarization-rotating waveguides," Optical engineering, 45 (4), 044603 (2006). doi:10.1117/1.2188408

[BUC12] Buckwalter, J.F.; Xuezhe Zheng; Guoliang Li; Raj, K.; Krishnamoorthy, A.V., "A Monolithic 25-Gb/s Transceiver With Photonic Ring Modulators and Ge Detectors in a 130-nm CMOS SOI Process," SolidState Circuits, IEEE Journal of , 47 (6), 1309,1322 (2012). doi: 10.1109/JSSC.2012.2189835

[CAS04] Castagnozzi, D., "Digital signal processing and electronic equalization (EE) of ISI," Optical Fiber Communication Conference (OFC 2004), 1, 797 (2004).

[CAV04] Cavallari, M.; Fludger, C. R S; Anslow, P.J., "Electronic signal processing for differential phase modulation formats," Optical Fiber Communication Conference (OFC 2004), 1, 422 (2004). 
[CHE07] Chen, L., Sherwood-Droz, N.; and Lipson, M., "Compact bandwidthtunable microring resonators," Optics Letters, 32(22), 3361-3363 (2007). doi:10.1364/OL.32.003361

[CHE11] Chen, L., Doerr, C. R., and Chen, Y.-K. (2011). "Compact polarization rotator on silicon for polarization-diversified circuits," Optics Letters, 36(4), 469-471 (2011). doi:10.1364/OL.36.000469

[CHO05] Chow, C.W.; and Tsang, H.K., "Polarization-independent DPSK demodulation using a birefringent fiber loop," Photonics Technology Letters, IEEE, 17 (6), 1313-1315 (2005). doi: 10.1109/LPT.2005.846566

[CIS] $\quad$ http://www.lightwaveonline.com/articles/2013/03/cisco-unveils-cpak$\underline{100 \mathrm{~g} \text {-silicon-photonics-based-optical-transcei.html }}$

[DAI05] Daikoku, M.; Yoshikane, N.; and Morita, I., "Performance comparison of modulation formats for $40 \mathrm{Gbit} / \mathrm{s}$ DWDM transmission systems," Optical Fiber Communication Conference (OFC/NFOEC2005) 5, 3, (2005). doi: 10.1109/OFC.2005.193155

[DAI11a] Daoxin Dai; Zhi Wang; and Bowers, J.E., "Considerations for the Design of Asymmetrical Mach-Zehnder Interferometers Used as Polarization Beam Splitters on a Submicrometer Silicon-On-Insulator Platform," Lightwave Technology, Journal of, 29 (12), 1808-1817 (2011). doi: 10.1109/JLT.2011.2142392

[DAI11b] Dai, D., \& Bowers, J. E. (2011). "Novel concept for ultracompact polarization splitter-rotator based on silicon nanowires," Optics Express, 19 (11), 10940-10949 (2011). doi:10.1364/OE.19.010940

[DIN11] Ding, Y., Xu, J., Peucheret, C., Pu, M., Liu, L., Seoane, J.; Ou, H.; Zhang, X; and Dexiu, H., "Multi-Channel 40 Gbit/s NRZ-DPSK Demodulation Using a Single Silicon Microring Resonator," Lightwave Technology, Journal of, 29 (5), 677-684 (2011). doi: 10.1109/JLT.2010.

[DOB08] De Dobbelaere, P.; Analui, B.; Balmater, E.; Guckenberger, D.; Harrison, M.; Koumans, R.; Kucharski, D.; Liang, Y.; Masini, G.; Mekis, A.; Mirsaidi, S.; Narasimha, A.; Peterson, M.; Pinguet, T.; Rines, D.; Sadagopan, V.; Sahni, S.; Sleboda, T.J.; Wang, Y.; Welch, B.; Witzens, J.; Yao, J.; Abdalla, S.; Gloeckner, S.; and Capellini, G., "Demonstration of first WDM CMOS photonics transceiver with monolithically integrated photo-detectors," Optical Communication (ECOC 2008), 34th European Conference on , 1-2, (2008). doi: 10.1109/ECOC.2008.4729209 
[DOB12] P. De Dobbelaere, S. Abdalla, S. Gloeckner, M. Mack, G. Masini, A. Mekis, T. Pinguet, S. Sahni, A. Narasimha, D. Guckenberger, and M. Harrison, "Si Photonics Based High-Speed optical Transceivers," in European Conference and Exhibition on Optical Communication (ECOC12), in 38th European Conference on , 1, (2012) paper We.1.E.5. doi:10.1364/ECEOC.2012.We.1.E.5

[DOE08] Doerr, C., Zhang, L., Buhl, L., Sinsky, J., Gnauck, A.; Winzer, P.; Adamiecki, A.; and Sauer, N., "High-Speed InP DQPSK Receiver," Post deadline paper in Optical Fiber Communication Conference (OFC2008),23, (2008).

[DOE10a] Doerr, C.R.; Winzer, P.J.; Chen, Young-kai; Chandrasekhar, S.; Rasras, M.S.; Chen, Long; Tsung-Yang Liow; Kah-Wee Ang; Guo-Qiang Lo, "Monolithic Polarization and Phase Diversity Coherent Receiver in Silicon," Lightwave Technology, Journal of , 28 (4), 520-525 (2010). doi: 10.1109/JLT.2009.2028656

[DOE10b] Doerr, C.; Zhang, L.; and Winzer, P., "Monolithic InP MultiWavelength Coherent Receiver," Post deadline in Optical Fiber Communication Conference (OFC2010), 26-28, (2010). doi:10.1364/OFC.2010.PDPB1

[DOE10c] Doerr, C. R., \& Chen, L. (2010). Monolithic PDM-DQPSK receiver in silicon. 36th European Conference and Exhibition on Optical Communication (pp. 1-3). IEEE.

Doerr, C.R.; and Chen, L., "Monolithic PDM-DQPSK receiver in silicon," in European Conference and Exhibition on Optical Communication (ECOC10), in 36th European Conference on , 1-3, (2010). doi: 10.1109/ECOC.2010.5621418

[DOE11] Doerr, C.R.; Buhl, L.L.; Baeyens, Y.; Aroca, R.; Chandrasekhar, S.; Liu, X.; Chen, L.; and Chen, Y. -K, "Packaged Monolithic Silicon 112-Gb/s Coherent Receiver," Photonics Technology Letters, IEEE , 23 (12), 762 764, (2011). doi: 10.1109/LPT.2011.2132699

[DOE12] Doerr, C.R.; Fontaine, N.K.; and Buhl, L.L., "PDM-DQPSK Silicon Receiver With Integrated Monitor and Minimum Number of Controls," Photonics Technology Letters, IEEE, 24 (8), 697-699, (2012). doi: 10.1109/LPT.2012.2187048

[DOI00] Doi, Y.; Fukushima, S.; Ohno, T.; Matsuoka, Yutaka; and Takeuchi, H., "Phase shift keying using optical delay modulation for millimeter-wave fiber-optic radio links," Lightwave Technology, Journal of , 18 (3), 301307, (2000). doi: 10.1109/50.827500 
[DON12a] Dong, P., Chen, L., Xie, C., Buhl, L. L.; and Chen, Y.-K, "50-Gb/s silicon quadrature phase-shift keying modulator," Optics Express, 20(19), 21181-21186 (2012). doi:10.1364/OE.20.021181

[DON12b] Dong, P.; Xie, C.; Chen, L.; Buhl, L. L.; and Chen, Y.-K., "112-Gb/s monolithic PDM-QPSK modulator in silicon," Optics Express, 20(26), 624-629 (2012). doi:10.1364/OE.20.00B624

[DON12c] Dong, P., Xie, C., Chen, L., Fontaine, N. K.; and Chen, Y., "Experimental demonstration of microring quadrature phase-shift keying modulators," Optics Letters, 37(7), 1178-1180 (2012). doi:10.1364/OL.37.001178

[DON13] Dong, P., Xie, C., Buhl, L. L.; and Chen, Y.K., "Silicon Microring Modulators for Advanced Modulation Formats" in Optical Fiber Communication Conference/National Fiber Optic Engineers Conference (OFC/NFOEC2013), 1, paper OW4J.2 (2013). doi:10.1364/OFC.2013.OW4J.2

[DUM04] Dumon, P.; Bogaerts, W.; Wiaux, V.; Wouters, J.; Beckx, S.; Van Campenhout, J.; Taillaert, D.; Luyssaert, B.; Bienstman, P.; Van Thourhout, D.; and Baets, R., "Low-loss SOI photonic wires and ring resonators fabricated with deep UV lithography," Photonics Technology Letters, IEEE , 16 (5), 1328-1330 (2004). doi: 10.1109/LPT.2004.826025

[EDI01] Edirisinghe, S.G.; Lepley, J.J.; and Siddiqui, A.S., "Polarization shaped duobinary transmission scheme," Photonics Technology Letters, IEEE, 13 (11), 1245-1247 (2001). doi: 10.1109/68.959378

[EFF11] Effenberger, F.J., "The XG-PON System: Cost Effective $10 \mathrm{~Gb} / \mathrm{s}$ Access," Lightwave Technology, Journal of, 29 (4), 403-409 (2011). doi: 10.1109/JLT.2010.2084989

[EPI] "ePIXfab: http://www.epixfab.eu/. “

[FÄR04] A. Färbert, S. Langenbach , N. Stojanovic, C. Dorschky , T. Kupfer , C. Schulien , J.-P. Elbers , H. Wernz , H. Griesser and C. Glingener, "Performance of a $10.7 \mathrm{~Gb} / \mathrm{s}$ receiver with digital equaliser using maximum likelihood sequence estimation," in proceedings of Optical Communication (ECOC 2004), 30th European Conference and Exhibition on, PD-Th4.1.5 (2004).

[FAR12] Faralli, S., Nguyen, K. N., Peters, J. D., Spencer, D. T., Blumenthal, D. J.; and Bowers, J. E, "Integrated hybrid Si/InGaAs $50 \mathrm{~Gb} / \mathrm{s}$ DQPSK 
receiver," Optics Express, 20(18), 19726-19734 (2012). doi:10.1364/OE.20.019726

[FEN10] Feng, N.-N., Liao, S., Feng, D., Dong, P., Zheng, D., Liang, H.; Shafiiha, R.; Li, G.; Cunningham, J. E; Krishnamoorthy, A.V; and Asghari, M , "High speed carrier-depletion modulators with $14 \mathrm{~V}-\mathrm{cm} \mathrm{V} \_\pi \mathrm{L}$ integrated on $0.25 \mu \mathrm{m}$ silicon-on-insulator waveguides," Optics Express, 18(8), 7994-7999 (2010). doi:10.1364/OE.18.007994

[FUK08] Fukuda, H., Yamada, K., Tsuchizawa, T., Watanabe, T.; Shinojima, H.; and Itabashi, S., "Polarization rotator based on silicon wire waveguides," Optics Express, 16(4), 2628-2635 (2008). doi:10.1364/OE.16.002628

[GAR09] Gardes, F. Y., Brimont, A., Sanchis, P., Rasigade, G., Marris-Morini, D., O’Faolain, L., Dong, F.; Fedeli, J. M. ; Dumon, P.; Vivien, L.; Krauss, T. F; Reed, G. R; and Martí, J, "High-speed modulation of a compact silicon ring resonator based on a reverse-biased pn diode,"

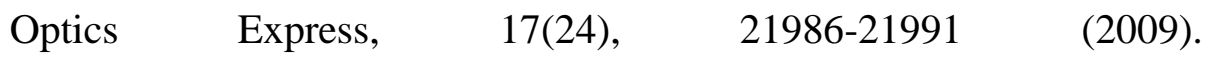
doi:10.1364/OE.17.021986

[GIT92] Gitlin, R; Hayes; J. F and Weinstein, S. B.,'Data Communications Principles". New York: Plenum, 1992.

[GNA03] Gnauck, A., "40-Gb/s RZ-differential phase shift keyed transmission," in Optical Fiber Communication Conference/National Fiber Optic Engineers Conference (OFC/NFOEC2003), 2, 450-451 (2003). doi: 10.1109/OFC.2003.1248333

[GNA05a] Gnauck, A.H.; Sinsky, J.; Winzer, P.J.; and Chandrasekhar, S., "Linear microwave-domain dispersion compensation of $10-\mathrm{Gb} / \mathrm{s}$ signals using heterodyne detection," in Optical Fiber Communication Conference/National Fiber Optic Engineers Conference (OFC/NFOEC2005), 6, 3 (2005). doi: 10.1109/OFC.2005.193209

[GNA05b] Gnauck, A. H.; and Winzer, P. J., "Optical phase-shift-keyed transmission," Lightwave Technology, Journal of , 23(1), 115-130 (2005). doi:10.1109/JLT.2004.840357

[GOI12] Goi, K., Oda, K., Kusaka, H., Terada, Y., Ogawa, K., Liow, T-Y.; Tu, $\mathrm{X}$; Lo, G-Q; and Kwong, D-L, "11-Gb/s 80-km transmission performance of zero-chirp silicon Mach-Zehnder modulator," Optics Express, 20(26), 350-356 (2012). doi:10.1364/OE.20.00B350

[GOI13a] Goi, K., Kusaka, H., Oka, A., Terada, Y., Ogawa, K., Liow, T.-Y., Tu, X.; Lo, X.; and Kwong, D-L, "20-Gb/s DPSK Transmission with 550ps/nm Dispersion Tolerance using Silicon Mach-Zehnder Modulator", 
in Optical Fiber Communication Conference/National Fiber Optic Engineers Conference (OFC/NFOEC2013), paper OW4J.5 (2013). doi:10.1364/OFC.2013.OW4J.5

[GOI13b] Goi, K.; Kusaka, H.; Oka, A.; Terada, Y.; Ogawa, K.; Liow, T.-Y.; Tu, X.; Lo, X.; and Kwong, D-L, "DQPSK/QPSK Modulation at 40-60 Gb/s using Low-Loss Nested Silicon Mach-Zehnder Modulator", in Optical Fiber Communication Conference/National Fiber Optic Engineers Conference (OFC/NFOEC2013), paper OW4J.4 (2013). doi:10.1364/OFC.2013.OW4J.4

[GRI01] Griffin, R.A.; Walker, R.G.; Johnstone, R. I.; Harris, R.; Perney, N. M B; Whitbread, N.D.; Widdowson, T.; and Harper, P., "Integrated $10 \mathrm{~Gb} / \mathrm{s}$ chirped return-to-zero transmitter using GaAs-AlGaAs modulators," in Optical Fiber Communication Conference/National Fiber Optic Engineers Conference (OFC/NFOEC2001), 4, 15 (2001).

[GRI02a] Griffin, R. A.; and Carter, A. C.,"Optical differential quadrature phaseshift key (oDQPSK) for high capacity optical transmission" in Optical Fiber Communication Conference/National Fiber Optic Engineers Conference (OFC/NFOEC2002), 367-368 (2002). doi:10.1109/OFC.2002.1036420

[GRI02b] Griffin, R.A.; Johnstone, R. I.; Walker, R.G.; Hall, J.; Wadsworth, S. D.; Berry, K.; Carter, A.C.; Wale, M.J.; Hughes, J.; Jerram, P. A.; and Parsons, N. J., "10 Gb/s optical differential quadrature phase shift key (DQPSK) transmission using GaAs/AlGaAs integration", in Optical Fiber Communication Conference/National Fiber Optic Engineers Conference (OFC/NFOEC2002), $1-3 \quad$ (2002). doi:10.1109/OFC.2002.1036787

[HAL11] Halir, R.; Roelkens, G.; Ortega-Moñux, A.; and Wangüemert-Pérez, J. G. High-performance $90^{\circ}$ hybrid based on a silicon-on-insulator multimode interference coupler," Optics Letters, 36(2), 178-180 (2011). doi:10.1364/OL.36.000178

[HEL] HELIOS Project, [Online]. Available: http://www.helios-project.eu/

[HO05] K.-P. Ho, "Phase-modulated Optical Communication Systems," (Springer, 2005)

[HOD02] Hodzic, A.; Konrad, B.; and Petermann, K., "Alternative modulation formats in $\mathrm{N} \times 40 \mathrm{~Gb} / \mathrm{s}$ WDM standard fiber RZ-transmission systems," Lightwave Technology, Journal of , 20 (4), 598-607 (2002). doi: $10.1109 / 50.996579$ 
[HUN84] Hunsperger, R. G., "Integrated Optics: Theory and Technology" (Springer-Verlag, Berlin, 1984).

[IBR07] Ibrahim, S. K, 'Study of Multilevel Modulation Formats for High Speed Digital Optical Communication Systems', Ph. D. thesis, University of Paderborn, Germany, 2007

[IDL04] Idler, W.; Klekamp, A.; Dischler, R.; Wedding, B., "Advantages of frequency shift keying in $10 \mathrm{~Gb} / \mathrm{s}$ systems," Advanced Modulation Formats, 2004 IEEE/LEOS Workshop on , 51-52 (2004). doi: 10.1109/ADMF.2004.1321716

[IHP] IHP - Innovations for High Performance Microelectronics "http://www.ihp-microelectronics.com/ "

[IME] Interuniversity Microelectronics Centre "http://www2.imec.be/be_en/home.html “

[INT13] http://newsroom.intel.com/community/intel_newsroom/blog/2013/01/1 6/intel-facebook-collaborate-on-future-data-center-rack-technologies

[INT] $\quad \underline{\text { Www.intel.com }}$

[INT11] Integlia, R. A; Yin, L.; Ding, D.; Pan, D. Z.; Gill, D. M.; and Jiang, W, "Parallel-coupled dual racetrack silicon micro-resonators for quadrature amplitude modulation" Optics Express, 19(16), 14892-14902 (2011). doi:10.1364/OE.19.014892

[ITU-987] ITU-T, "Recommendation G.987.3," [Online]. Available www.itu.int/rec/T-REC-G.987.3/en

[JAC94] Jocabsen, G., "Noise in digital optical transmission systems," 1994 (Artech HouseBoston, MA)

[JAL06] Jalali, B.; and Fathpour, S., "Silicon Photonics". Lightwave Technology, Journal of, 24(12), 4600-4615 (2006). doi:10.1109/JLT.2006.885782

[JEN07] Jensen, J.B.; Tokle, T.; Peucheret, C.; Jeppesen, P., "Transmission of Multilevel $60 \mathrm{Gbit} / \mathrm{s}$ Polarization Multiplexed RZ-D8PSK using only 10 Gbit/s equipment," in Optical Fiber Communication Conference/National Fiber Optic Engineers Conference (OFC/NFOEC2007), 1-3. doi:10.1109/OFC.2007.4348907

[KAT09] Katigbak, A.; J. F. Strother, Jr.; J. Lin, "Compact silicon slot waveguide polarization splitter," Optical Engineering, 48(8), 080503. doi:10.1117/1.3200898 
[KAW90] Kawachi, M., "Silica waveguides on silicon and their application to integrated-optic components," Optical and Quantum Electronics, 22(5), 391-416 (1990). doi:10.1007/BF02113964

[KIK10] Kikuchi, N.; and Sasaki, S., "Highly Sensitive Optical Multilevel Transmission of Arbitrary Quadrature-Amplitude Modulation (QAM) Signals With Direct Detection," Lightwave Technology, Journal of, 28 (1), 123-130 (2010). doi: 10.1109/JLT.2009.2035827

[KIM03] Kim, H.; Essiambre, R., "Transmission of 8 × $20 \mathrm{~Gb} / \mathrm{s}$ DQPSK signals over $310-\mathrm{km}$ SMF with $0.8-\mathrm{b} / \mathrm{s} / \mathrm{Hz}$ spectral efficiency," Photonics Technology Letters, IEEE, 15(5), 769-771 (2003). doi:10.1109/LPT.2003.809983

[KIM04] Kim, H., "Differential phase shift keying for $10-\mathrm{Gb} / \mathrm{s}$ and 40-Gb/s systems," Advanced Modulation Formats, 2004 IEEE/LEOS Workshop on, 13-14 (2004). doi: 10.1109/ADMF.2004.1321697

[KOO06] Koonath, P.; Indukuri, T.; and Jalali, B., "Monolithic 3-D silicon photonics," Lightwave Technology, Journal of, 24 (4), 1796-1804 (2006). doi: 10.1109/JLT.2006.871121

[KOT] www.kotura.com

[KOT13] http://www.kotura.com/news-events/pr051513.html

[KRA03] Kramer, G.; Ashikhmin, A.; Van Wijngaarden, A.J.; and Xing Wei, "Spectral efficiency of coded phase-shift keying for fiber-optic communication," Lightwave Technology, Journal of, 21 (10), 24382445 (2003). doi: 10.1109/JLT.2003.817704

[KRO11] Kroh, M.; Zimmermann, L.; Bach, H. -G; Voigt, K.; Beling, A.; Nielsen, M.L.; Ludwig, R.; Bruns, J.; and Unterbörsch, G., "Integrated receivers on silicon-on-insulator for advanced modulation formats," Optoelectronics, IET, 5 (5), 211-217 (2011). doi: 10.1049/ietopt.2010.0059

[LEE06] Lee, B. G.; Small, B.A; Bergman, K.; Xu, Q.; and Lipson, M., "Transmission of high-data-rate optical signals through a micrometerscale silicon ring resonator," Optics Letters, 31(18), 2701-2703 (2006). doi:10.1364/OL.31.002701

[LEP00] Lepley, J.J.; Ellison, J.G.; Edirisinghe, S.G.; Siddiqui, A. S.; Walker, S.D., "Excess penalty impairments of polarisation shift keying transmission format in presence of polarisation mode dispersion," Electronics Letters , 36(8), 736-737 (2000). doi:10.1049/el:20000551 
[LIA05a] Liao, L.; Samara-Rubio, D.; Morse, M.; Liu, A.; Hodge, D.; Rubin, D.; Keil, U. D.; and Franck, T, "High speed silicon Mach-Zehnder modulator," Optics Express, 13(8), 3129-3135 (2005). doi:10.1364/OPEX.13.003129

[LIA05b] Liang, T.K.; Tsang, H.K., "Integrated polarization beam splitter in high index contrast silicon-on-insulator waveguides," Photonics Technology Letters, IEEE, 17 (2), 393-395 (2005). doi: 10.1109/LPT.2004.839462

[LIG] http://www.lightwaveonline.com/articles/2012/02/cisco-to-acquirecmos-silicon-photonics-firm-lightwire.html

[LIP06] Lipson, M., "Compact Electro-Optic Modulators on a Silicon Chip," Selected Topics in Quantum Electronics, IEEE Journal of, 12 (6), 15201526 (2006). doi: 10.1109/JSTQE.2006.885341

[LIU11a] Liu, L.; Ding, Y.; Yvind, K.; and Hvam, J. M., "Efficient and compact TE-TM polarization converter built on silicon-on-insulator platform with a simple fabrication process," Optics Letters, 36(7), 1059-1061 (2011). doi:10.1364/OL.36.001059

[LIU11b] Liu, L.; Ding, Y.; Yvind, K.; and Hvam, J. M., "Silicon-on-insulator polarization splitting and rotating device for polarization diversity circuits," Optics Express, 19(13), 12646-12651 (2011). doi:10.1364/OE.19.012646

[MAN10] Manipatruni, S.; Chen, L.; and Lipson, M., "Ultra high bandwidth WDM using silicon microring modulators," Optics Express, 18(16), 1685816867 (2010). doi:10.1364/OE.18.016858

[MAR06] Marris-Morini, D.; Le Roux, X.; Vivien, L.; Cassan, E.; Pascal, D.; Halbwax, M.; Maine, S.; Laval, S; Fédéli, J. M; and Damlencourt, J. F , "Optical modulation by carrier depletion in a silicon PIN diode," Optics Express, 14(22), 10838-10843 (2006). doi:10.1364/OE.14.010838

[McG05] McGhan, D.; Laperle, C.; Savehenko, A.; Chuandong Li; Mak, G.; O'Sullivan, M., "5120 km RZ-DPSK transmission over G652 fiber at 10 $\mathrm{Gb} / \mathrm{s}$ with no optical dispersion compensation," in Optical Fiber Communication Conference/National Fiber Optic Engineers Conference (OFC/NFOEC2005), 5, 3. doi:10.1109/OFC.2005.193205

[MIL09] Miller, D.A.B., "Device Requirements for Optical Interconnects to Silicon Chips," Proceedings of the IEEE, 97 (7), 1166-1185 (2009). doi: 10.1109/JPROC.2009.2014298

[MIL13] Milivojevic, B.; Raabe, C.; Shastri, A.; Webster, M.; Metz, P.; Sunder, S.; Chattin, B.; Wiese, S.; Dama, B.; and Shastri, K.; "112Gb/s DP- 
QPSK Transmission Over 2427km SSMF Using Small-Size Silicon Photonic IQ Modulator and Low-Power CMOS Driver," in Optical Fiber Communication Conference/National Fiber Optic Engineers Conference (OFC/NFOEC2013), paper OTh1D.1. doi:10.1364/OFC.2013.OTh1D.1

[MIN] Commissariat à l'énergie atomique - Laboratoire des technologies de L'information « http://www.minatec.com/. "

[MIZ03] Mizuochi, T.; Kubo, K.; Yoshida, H.; Fujita, H.; Tagami, H.; Akita, M.; Motoshima, K., "Next generation FEC for optical transmission systems," in Optical Fiber Communication Conference/National Fiber Optic Engineers Conference (OFC/NFOEC2003), 2, 527-528. doi: 10.1109/OFC.2003.124838

[MO05] Mo, J.; Dong, Y.; Wen, Y.; Takahashi, S.; Wang, Y.; and Lu, C., "Optical minimum-shift keying modulator for high spectral efficiency WDM systems," in proceedings of Optical Communication (ECOC 2005), 31st European Conference and Exhibition on, 4, 781-782 (2005).

[NAG11] Nagarajan, R.; Rahn, J.; Kato, M.; Pleumeekers, J.; Lambert, D.; Lal, V.; Huan-Shang Tsai; Nilsson, A.; Dentai, A.; Kuntz, M.; Malendevich, R.; Jie Tang; Jiaming Zhang; Butrie, T.; Raburn, M.; Little, B.; Wei Chen; Goldfarb, G.; Dominic, V.; Taylor, B.; Reffle, M.; Kish, F.; Welch, D., "10 Channel, 45.6 Gb/s per Channel, PolarizationMultiplexed DQPSK, InP Receiver Photonic Integrated Circuit," Lightwave Technology, Journal of, 29 (4), 386-395 (2011). doi: 10.1109/JLT.2010.2096555

[NAR07] Narasimha, A.; Analui, B.; Yi Liang; Sleboda, T.J.; Abdalla, S.; Balmater, E.; Gloeckner, S.; Guckenberger, D.; Harrison, M.; Koumans, R. G. M. P.; Kucharski, D.; Mekis, A.; Mirsaidi, S.; Dan Song; and Pinguet, T., "A Fully Integrated $4 \times 10-\mathrm{Gb} / \mathrm{s}$ DWDM Optoelectronic Transceiver Implemented in a Standard $0.13 \mu \mathrm{m}$ CMOS SOI Technology," Solid-State Circuits, IEEE Journal of, 42 (12), 2736-2744 (2007). doi: 10.1109/JSSC.2007.908713

[NAW06] Nawrocka, M.S.; Tao Liu; Xuan Wang; and Panepucci, R.R., "Tunable silicon microring resonator with wide free spectral range," Applied Physics Letters, 89 (7), 071110-071110-3 (2006). doi: $10.1063 / 1.2337162$

[NIE05] Nielsen, T.; and Chandrasekhar, S., "OFC 2004 workshop on optical and electronic mitigation of impairments," Lightwave Technology, Journal of, 23 (1), 131-142 (2005). doi: 10.1109/JLT.2004.840342(410) 23 
[OGA12] Ogawa, K.; Goi, K.; Kusaka, H.; Oda, K.; Tsung-Yang Liow; Xiaoguang Tu; Guo-Qiang Lo; and Dim-Lee Kwong, "20-Gbps silicon photonic waveguide nested Mach-Zehnder QPSK modulator," in Optical Fiber Communication Conference/National Fiber Optic Engineers Conference (OFC/NFOEC2012), 1-3. doi:10.1364/NFOEC.2012.JTh2A.20

[OHM04a] Ohm, M., "Optical 8-DPSK and receiver with direct detection and multilevel electrical signals," Advanced Modulation Formats, 2004 IEEE/LEOS Workshop on, 45-46 (2004). doi: 10.1109/ADMF.2004.1321713

[OHM04b] Ohm, M.; and Freckmann, T., "Comparison of different DQPSK transmitters with NRZ and RZ impulse shaping," Advanced Modulation Formats, 2004 IEEE/LEOS Workshop on, 7-8 (2004). doi: 10.1109/ADMF.2004.1321694

[OZE01] Ozeki, Y.; Higuma, K.; Oikawa, S.; Kishi, M.; and Tsuchiya, M., "A 60$\mathrm{GHz}$ optoelectronic mixing scheme of high image and carrier rejection ratios with an integrated optical single-sideband modulator employed," Microwave Theory and Techniques, IEEE Transactions on, 49 (10), 1986-1991 (2001). doi: 10.1109/22.954818

[PAD11] Padmaraju, K.; Ophir, N.; Qianfan Xu; Schmidt, B.; Shakya, J.; Manipatruni, S.; Lipson, M.; and Bergman, K., "Error-free transmission of DPSK at $5 \mathrm{~Gb} / \mathrm{s}$ using a silicon microring modulator," Optical Communication (ECOC2011), 37th European Conference and Exhibition on, 1-3 (2011).

[PAR11] Hyundai Park; Sysak, M.N.; Hui-Wen Chen; Fang, A.W.; Di Liang; Ling Liao; Koch, B.R.; Bovington, J.; Yongbo Tang; Wong, K.; JacobMitos, M.; Jones, R.; and Bowers, J.E., "Device and Integration Technology for Silicon Photonic Transmitters," Selected Topics in Quantum Electronics, IEEE Journal of, 17 (3), 671-688 (2011). doi: 10.1109/JSTQE.2011.2106112

[PAV04] Pavesi, L.; and Lockwood, D. J., "Silicon Photonics" (Springer, New York, 2004).

[PAW96] Pawlowski, E.; Takiguchi, K.; Okuno, M.; Sasayama, K.; Himeno, A.; Okamoto, K.; Ohmori, Y., "Variable bandwidth and tunable centre frequency filter using transversal-form programmable optical filter," Electronics Letters, 32 (2), 113-114 (1996). doi: 10.1049/el:19960095

[PHO12] Commercial software by Photon Design (www.photond.com). 
[PIN08] Pinguet, T.; Analui, B.; Balmater, E.; Guckenberger, D.; Harrison, M.; Koumans, R.; Kucharski, D.; Liang, Y.; Masini, G.; Mekis, A.; Mirsaidi, S.; Narasimha, A.; Peterson, M.; Rines, D.; Sadagopan, V.; Sahni, S.; Sleboda, T.J.; Song, D.; Wang, Y.; Welch, B.; Witzens, J.; Yao, J.; Abdalla, S.; Gloeckner, S.; de Dobbelaere, P.; and Capellini, G., "Monolithically integrated high-speed CMOS photonic transceivers," Group IV Photonics (GFP2008), 5th IEEE International Conference on, 362-364 (2008). doi: 10.1109/GROUP4.2008.4638200

[POO04] Poon, J.; Scheuer, J.; Mookherjea, S.; Paloczi, G. T.; Huang, Y.; and Yariv, A., "Matrix analysis of microring coupled-resonator optical waveguides," Optics Express, 12(1), 90-103 (2004). doi:10.1364/OPEX.12.000090

[POP07] Popovic, M.; Barwicz, T.; Gan, F.; Dahlem, M.; Holzwarth, C.; Rakich, P.; Smith, H.; Ippen, E.; and Kärtner, F., "Transparent Wavelength Switching of Resonant Filters," post deadline in Conference on Lasers and Electro-Optics/Quantum Electronics and Laser Science Conference and Photonic Applications Systems Technologies (CLEO2007), paper CPDA2.

[QIA11] Qian, D.; Fan, S-H; Cvijetic, N.; Hu, J.; and Wang, T., "64/32/16QAMOFDM using direct-detection for 40G-OFDMA-PON downstream," in Optical Fiber Communication Conference/National Fiber Optic Engineers Conference (OFC/NFOEC2011)1,3 (2011).

[RAB07] Rabus, D. G, "Integrated Ring Resonators: The Compendium" (Springer, Berlin, 2007).

[RAI150] Raith 150 e-beam lithography software http://www.nanophys.kth.se/nanophys/facilities/nfl/manual/.

[RAS09] Rasras, M.S.; Kun-Yii Tu; Gill, D.M.; Chen, Young-kai; White, A.E.; Patel, S.S.; Pomerene, A.; Carothers, D.; Beattie, J.; Beals, M.; Michel, J.; and Kimerling, L.C., "Demonstration of a Tunable MicrowavePhotonic Notch Filter Using Low-Loss Silicon Ring Resonators," Lightwave Technology, Journal of, 27 (12), 2105-2110 (2009). doi: 10.1109/JLT.2008.2007748

[REE04] G. T. Reed and A. P. Knights, "Silicon Photonics: An Introduction," John Wiley \& Sons, Inc., 2004.

[REI06] Reitzenstein, S.; Bazhenov, A.; Gorbunov, A.; Hofmann, C.; Munch, S.; Loffler, A.; Kamp, M.; Reithmaier, J. -P; Kulakovskii, V. D.; and Forchel, A., "Lasing in high-Q quantum-dot micropillar cavities," 
Applied Physics Letters, 89 (5), 051107-051107-3 (2006). doi: $10.1063 / 1.2266231$

[ROE08] Roelkens, G.; Vermeulen, D.; Van Thourhout, D.; Baets, R.; Brision, S.; Lyan, P.; Gautier, P.; Fedeli, J.-M., "High efficiency diffractive grating couplers for interfacing a single mode optical fiber with a nanophotonic silicon-on-insulator waveguide circuit," Applied Physics Letters , 92 (13), 131101,131101-3 (2008). doi: 10.1063/1.2905260

[RSO00] Rsoft Design Group, Inc., 2000 Executive Group Blvd. Ossining, NY 10562, www.rsoftdesign.com.

[SAK05] Sakamoto, T.; Kawanishi, T.; Miyazaki, T.; and Izutsu, M., "Novel modulation scheme for optical continuous-phase frequency-shift keying," in Optical Fiber Communication Conference/National Fiber Optic Engineers Conference (OFC/NFOEC2005), 5, 3 (2005).

doi: 10.1109/OFC.2005.193106

[SCH12] Schmogrow, R.; Nebendahl, B.; Winter, M.; Josten, A.; Hillerkuss, D.; Koenig, S.; Meyer, J.; Dreschmann, M.; Huebner, M.; Koos, C.; Becker, J.; Freude, W.; and Leuthold, J., "Corrections to "Error Vector Magnitude as a Performance Measure for Advanced Modulation Formats" [Jan 1, 2012 61-63]," Photonics Technology Letters, IEEE, 24 (23), 2198-2198 (2012). doi: 10.1109/LPT.2012.2219471

[SHA06] Shafik, R.A.; Rahman, S.; and Islam, R., "On the Extended Relationships Among EVM, BER and SNR as Performance Metrics," Electrical and Computer Engineering, 2006. ICECE '06. International Conference on, 408-411 (2006). doi: 10.1109/ICECE.2006.355657

[SHA08] Shacham, A.; Bergman, K.; Carloni, L.P., "Photonic Networks-on-Chip for Future Generations of Chip Multiprocessors," Computers, IEEE Transactions on, 57 (9), 1246-1260 (2008). doi: 10.1109/TC.2008.78

[SHE08] Sherwood-Droz, N.; Wang, H.; Chen, L.; Lee, B. G.; Biberman, A.; Bergman, K.; and Lipson, M., "Optical 4x4 hitless silicon router for optical networks-on-chip (NoC)," Optics Express, 16(20), 15915-15922 (2008). doi:10.1364/OE.16.015915

[SHI07] Shi, Y.; Dai, D.; and He. S, "Proposal for an Ultracompact PolarizationBeam Splitter Based on a Photonic-Crystal-Assisted Multimode Interference Coupler," Photonics Technology Letters, IEEE, 19 (11), 825-827 (2007). doi: 10.1109/LPT.2007.897297

[SID02] Shamim Siddiqui, A.; Edirisinghe, S.G.; Lepley, J.J.; Ellison, J.G.; and Walker, S.D., "Dispersion-tolerant transmission using a duobinary 
polarization-shift keying transmission scheme," Photonics Technology Letters, IEEE, 14 (2), 158-160 (2002). doi: 10.1109/68.980484

[SLE11] Sleiffer, V.A.J.M.; Al Fiad, M.S.A.S.; Borne, D. van den; Kuschnerov, M.; Veljanovski, V.; Hirano, M.; Yamamoto, Y.; Sasaki, T.; Jansen, S.L.; Wuth, T.; and Waardt, H. de , "10x224-Gb/s POLMUX-16QAM transmission over $656 \mathrm{~km}$ of large-Aeff PSCF with a special efficiency of $5.6 \mathrm{~b} / \mathrm{s} / \mathrm{Hz}$," Photonics Technology Letters, IEEE, 23(20), 1427-1429 (2011). doi:10.1109/LPT.2011.2162722

[SOL95] Soldano, L.B.; and Pennings, E. C M, "Optical multi-mode interference devices based on self-imaging: principles and applications," Lightwave Technology, Journal of, 13 (4), 615-627 (1995). doi: 10.1109/50.372474

[SOT12] Sotiropoulos, N.; Koonen, T.; and De Waardt, H., "TDM-PON with 30 $\mathrm{Gb} / \mathrm{s}$ D8PSK downstream and $10 \mathrm{~Gb} / \mathrm{s}$ OOK upstream based on a digital incoherent receiver," Optics Express, 20(27), 29096-29104 (2012). doi:10.1364/OE.20.029096

[SOT13] Sotiropoulos, N, 'Advanced Modulation Formats for Optical Access Networks', Ph. D. thesis, Eindhoven University of Technology, Holland, 2013

[SPE08] Spector, S. J.; Geis, M. W.; Zhou, G.-R.; Grein, M. E.; Gan, F.; Popovic, M. A; Yoon, J. U.; Lennon, D. M.; Ippen, E. P; Kärtner, F. Z; and Lyszczarz, T. M, "CMOS-compatible dual-output silicon modulator for analog signal processing," Optics Express, 16(15), 11027-11031 (2008). doi:10.1364/OE.16.011027

[STE10] Steer, M.; "Microwave and RF Design: A Systems Approach," First Edition, (c) (2010).

ISBN 9781891121883

[SUZ12] Suzuki, K.; Nguyen, H. C.; Tamanuki, T.; Shinobu, F.; Sakai, Y.; and Baba, T, " Slow-light-based variable symbol-rate silicon photonics DQPSK receiver,” Optics Express, 20(4), 4796-4804 (2012). doi:10.1364/OE.20.004796

[TAI02] Taillaert, D.; Bogaerts, W.; Bienstman, P.; Krauss, T.F.; van Daele, P.; Moerman, I.; Verstuyft, S.; De Mesel, K.; and Baets, R., "An out-ofplane grating coupler for efficient butt-coupling between compact planar waveguides and single-mode fibers," Quantum Electronics, IEEE Journal of, 38 (7), 949-955 (2002). doi: 10.1109/JQE.2002.1017613

[THO12] Thomson, D.J.; Gardes, F.Y.; Fedeli, J.-M.; Zlatanovic, S.; Youfang Hu; Kuo, B. P -P; Myslivets, E.; Alic, N.; Radic, S.; Mashanovich, G.Z.; and 
Reed, G.T., "50-Gb/s Silicon Optical Modulator," Photonics Technology Letters, IEEE, 24 (4), 234-236 (2012). doi: 10.1109/LPT.2011.2177081

[TOK04] Tokle, T.; Davidson, C.R.; Nissov, M.; Cai, J.-X.; Foursa, D.;and Pilipetskii, A., "6500 km transmission of RZ-DQPSK WDM signals," Electronics Letters, 40 (7), 44-,445 (2004). doi: 10.1049/el:20040274

[TSU05] Tsukamoto, S.; Ly-Gagnon, D.; Katoh, K.; and Kikuchi, K., "Coherent demodulation of 40-Gbit/s polarization-multiplexed QPSK signals with $16-\mathrm{GHz}$ spacing after 200-km transmission," in Optical Fiber Communication Conference/National Fiber Optic Engineers Conference (OFC/NFOEC2005), 6, 3 (2005). doi: 10.1109/OFC.2005.193207

[TU10] Tu, X.; Ang, S. S. N.; Chew, A. B.; Teng, J.; and Mei, T, "An Ultracompact Directional Coupler Based on GaAs Cross-Slot Waveguide," Photonics Technology Letters, IEEE, 22 (17), 1324-1326 (2010). doi: 10.1109/LPT.2010.2055234

[VAN07] Van Laere, F., Bogaerts, W., Taillaert, D., Dumon, P., Van Thourhout, D., \&Baets, R. (2007). Compact Focusing Grating Couplers Between Optical Fibers and Silicon-on-Insulator Photonic Wire Waveguides. OFC/NFOEC 2007 - 2007 Conference on Optical Fiber Communication and the National Fiber Optic Engineers Conference (Vol. 19, pp. 1-3). IEEE. doi:10.1109/OFC.2007.4348869Van Laere, F.; Bogaerts, W.; Taillaert, D.; Dumon, P.; Van Thourhout, D.; Baets, R., "Compact Focusing Grating Couplers Between Optical Fibers and Silicon-onInsulator Photonic Wire Waveguides," in Optical Fiber Communication Conference/National Fiber Optic Engineers Conference (OFC/NFOEC2007), 1-3 (2007). doi: 10.1109/OFC.2007.4348869

[VAS91] Vassallo, C., "Optical Waveguide Concepts" (Elsevier, Amsterdam, 1991).

[VEL12] Velasco, A. V; Calvo, M. L.; Cheben, P.; Ortega-Moñux, A.; Schmid, J. H.; Ramos, C. A.; Fernandez, Í. M.; Lapointe, J.; Vachon, M.; Janz, S.; and $\mathrm{Xu}, \mathrm{D}$., "Ultracompact polarization converter with a dual subwavelength trench built in a silicon-on-insulator waveguide," Optics Letters, 37(3), 365-367 (2012). doi:10.1364/OL.37.000365

[VER12] Vermeulen, D.; Selvaraja, S.; Verheyen, P.; Absil, P.; Bogaerts, W.; Van Thourhout, D.; and Roelkens, G., "Silicon-on-Insulator Polarization Rotator Based on a Symmetry Breaking Silicon Overlay," Photonics Technology Letters, IEEE, 24 (6), 482-484 (2012). doi: 10.1109/LPT.2011.2181944 
[VIV12] Vivien, L.; Polzer, A.; Marris-Morini, D.; Osmond, J.; Hartmann, J. M.; Crozat, P.; Cassan, E.; Kopp, C.; Zimmermann, H.; and Fédéli, J.M, "Zero-bias 40Gbit/s germanium waveguide photodetector on silicon," Optics Express, 20(2), 1096-1101 (2012). doi:10.1364/OE.20.001096

[WAN04] Wang, J.; Kahn, J.M., "Impact of chromatic and polarization-mode dispersions on DPSK systems using interferometric demodulation and direct detection," Lightwave Technology, Journal of, 22 (2), 362-371 (2004). doi: 10.1109/JLT.2003.822101

[WAN08] Wang, Z.; and Dai, D, "Ultrasmall Si-nanowire-based polarization rotator". Journal of the Optical Society of America B, 25(5), 747-753 (2008). doi:10.1364/JOSAB.25.000747

[WAN13] Wang, J.; Liang, D.; Tang, Y.; Dai, D.; and Bowers, J. E., "Realization of an ultra-short silicon polarization beam splitter with an asymmetrical bent directional coupler," Optics Letters, 38(1), 4-6 (2013). doi:10.1364/OL.38.000004

[WAT08] Watanabe, K.; Hashizume, Y.; Nasu, Y.; Kohtoku, M.; Itoh, M.; and Inoue, Y., "Ultralow Power Consumption Silica-Based PLCVOA/Switches," Lightwave Technology, Journal of, 26 (14), 2235-2244 (2008). doi: 10.1109/JLT.2008.920542

[WEL07] Welch, D.F.; Kish, F.A.; Melle, S.; Nagarajan, R.; Kato, M.; Joyner, C.H.; Pleumeekers, J.L.; Schneider, R.P.; Back, J.; Dentai, A.G.; Dominic, V.G.; Evans, P.W.; Kauffman, M.; Lambert, D. J H; Hurtt, S.K.; Mathur, A.; Mitchell, M.L.; Missey, M.; Murthy, S.; Nilsson, A.C.; Salvatore, Randal A.; Van Leeuwen, M.F.; Webjorn, J.; Ziari, M.; Grubb, S.G.; Perkins, D.; Reffle, M.; and Mehuys, D.G., "Large-Scale InP Photonic Integrated Circuits: Enabling Efficient Scaling of Optical Transport Networks," Selected Topics in Quantum Electronics, IEEE Journal of, 13 (1), 22-31 (2007). doi: 10.1109/JSTQE.2006.890068

[WIB05] Wiberg, A.; Perez-Millan, P.; Andres, M.V.; Andrekson, P.A.; and Hedekvist, P-O, "Fiber-optic 40-GHz mm-wave link with $2.5-\mathrm{Gb} / \mathrm{s}$ data transmission," Photonics Technology Letters, IEEE, 17 (9), 1938-1940 (2005). doi: 10.1109/LPT.2005.853035

[WIN06] Winzer, P.J.; and Essiambre, R., "Advanced Optical Modulation Formats," Proceedings of the IEEE, 94 (5), 952-985 (2006). doi: 10.1109/JPROC.2006.873438

[XIA07a] Xia, F.; Rooks, M.; Sekaric, L.; and Vlasov, Y., "Ultra-compact high order ring resonator filters using submicron silicon photonic wires for 
on-chip optical interconnects," Optics Express, 15(19), 11934-11941 (2007). doi:10.1364/OE.15.011934

[XIA07b] Xiao, S.; Khan, M. H.; Shen, H.; and Qi, M., "Multiple-channel silicon micro-resonator based filters for WDM applications," Optics Express, 15(12), 7489-7498 (2007). doi:10.1364/OE.15.007489

[XIA08] Xiao, J.; Liu, X.; and Sun, X., "Design of a Compact Polarization Splitter in Horizontal Multiple-Slotted Waveguide Structures," Japanese Journal of Applied Physics, 47(5), 3748-3754 (2008). doi:10.1143/JJAP.47.3748

[XU12] Xu, K.; Cheng, Z.; Wong, C. Y.; and Tsang, H. K., "Tunable integrated variable bit-rate DPSK silicon receiver," Optics Letters, 37(22), 47384740 (2012). doi:10.1364/OL.37.004738

[YAN09] Yang, B.; Shin, S-Y.; and Zhang, D., "Ultrashort Polarization Splitter Using Two-Mode Interference in Silicon Photonic Wires," Photonics Technology Letters, IEEE, 21 (7), $432-434$ (2009). doi: 10.1109/LPT.2009.2013638

[YAO07] Yao, J.; Leuenberger, D.; Lee, M.-C. M.; and Wu, M. C, "Silicon Microtoroidal Resonators With Integrated MEMS Tunable Coupler," Selected Topics in Quantum Electronics, IEEE Journal of, 13 (2), 202208 (2007). doi: 10.1109/JSTQE.2007.893743

[YAR02] Yariv, A., "Critical coupling and its control in optical waveguide-ring resonator systems," Photonics Technology Letters, IEEE, 14 (4), 483485 (2002). doi: 10.1109/68.992585

[YOS05] Yoshikane, N.; and Morita, I., " $1.14 \mathrm{~b} / \mathrm{s} / \mathrm{Hz}$ spectrally efficient 50/spl times/85.4-Gb/s transmission over $300 \mathrm{~km}$ using copolarized RZDQPSK signals", Lightwave Technology, Journal of, 23(1), 108-114 (2005).doi: 10.1109/JLT.2004.840343(410) 23

[ZHA08] Zhang, L.; Yang, J.-Y.; Li, Y.; Beausoleil, R. G.; and Willner, A. E., "Monolithic modulator and demodulator of differential quadrature phase-shift keying signals based on silicon microrings," Optics Letters, 33(15), 1428-1430 (2008). doi:10.1364/OL.33.001428

[ZHA10a] Zhang, L.; Li, Y.; Yang, J. -Y; Song, M.; Beausoleil, R.G.; and Willner, A.E., "Silicon-Based Microring Resonator Modulators for Intensity Modulation," Selected Topics in Quantum Electronics, IEEE Journal of, 16 (1), 149-158 (2010). doi: 10.1109/JSTQE.2009.2027816

[ZHA10b] Zhang, J.; Yu, M.; Lo, G. P.; and Kwong, D., "Silicon-WaveguideBased Mode Evolution Polarization Rotator," Selected Topics in 
Quantum Electronics, IEEE Journal of, 16 (1), 53-60 (2010). doi: 10.1109/JSTQE.2009.2031424

[ZHE10] Zheng, X.; Shubin, I.; Li, G.; Pinguet, T.; Mekis, A.; Yao, J.; Thacker, H.; Luo, Y.; Costa, J.; Raj, K.; Cunningham, J.; and Krishnamoorthy, A.,"A tunable $1 \times 4$ silicon CMOS photonic wavelength multiplexer/demultiplexer for dense optical interconnects," Optics Express, 18(5), 5151-5160 (2010). doi:10.1364/OE.18.005151 